LA-14256-SR

Status Report

Approved for public release;

distribution is unlimited.

\title{
Emissions Inventory Report Summary for \\ Los Alamos National Laboratory for \\ Calendar Year 2004
}

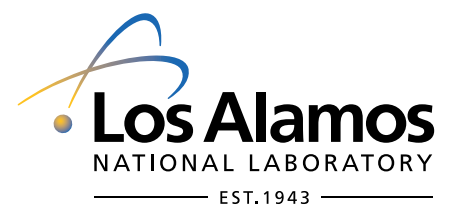

The World's Greatest Science Protecting America 


\section{Edited by Hector Hinojosa, Group IM-1}

Los Alamos National Laboratory, an affirmative action/equal opportunity employer, is operated by the University of California for the United States Department of Energy under contract W-7405-ENG-36.

This report was prepared as an account of work sponsored by an agency of the United States Government. Neither the Regents of the University of California, the United States Government nor any agency thereof, nor any of their employees make any warranty, express or implied, or assume any legal liability or responsibility for the accuracy, completeness, or usefulness of any information, apparatus, product, or process disclosed, or represent that its use would not infringe privately owned rights. Reference herein to any specific commercial product, process, or service by trade name, trademark, manufacturer, or otherwise does not necessarily constitute or imply its endorsement, recommendation, or favoring by the Regents of the University of California, the United States Government, or any agency thereof. The views and opinions of authors expressed herein do not necessarily state or reflect those of the Regents of the University of California, the United States Government, or any agency thereof. Los Alamos National Laboratory strongly supports academic freedom and a researcher's right to publish; as an institution, however, the Laboratory does not endorse the viewpoint of a publication or guarantee its technical correctness. 
LA-14256-SR

Status Report

Issued: October 2005

\title{
Emissions Inventory Report Summary for Los Alamos National Laboratory for
}

Calendar Year 2004

\author{
Meteorology and Air Quality Group (ENV-MAQ)
}





\section{CONTENTS}

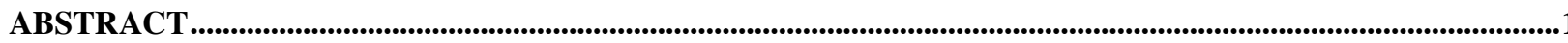

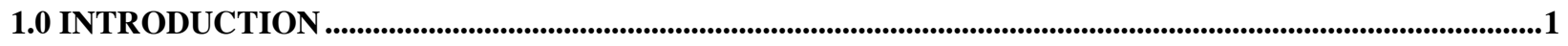

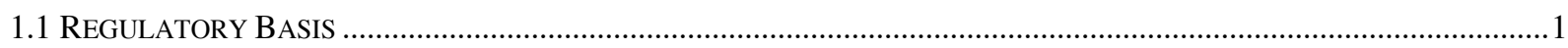

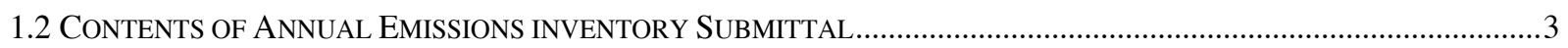

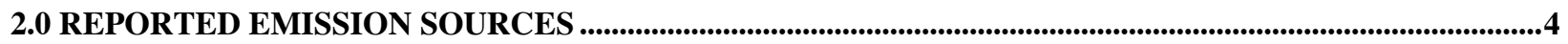

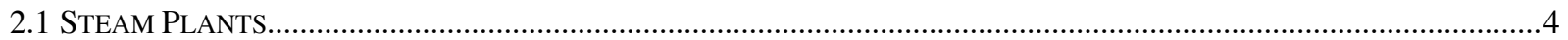

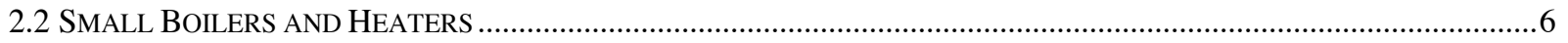

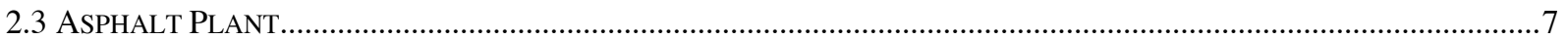

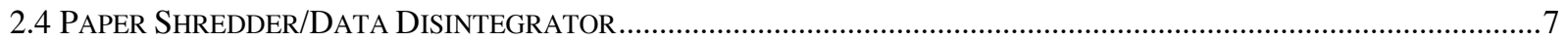

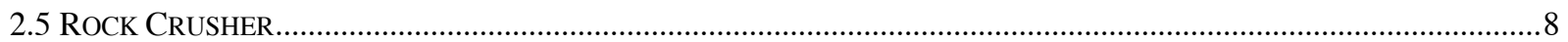

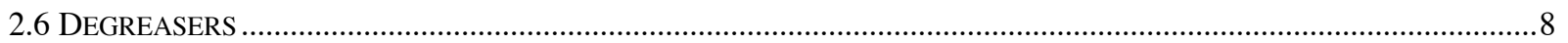

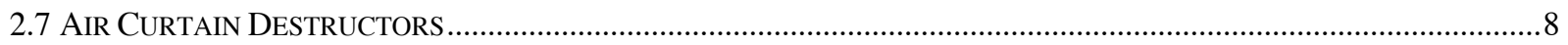

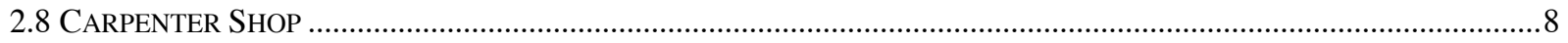

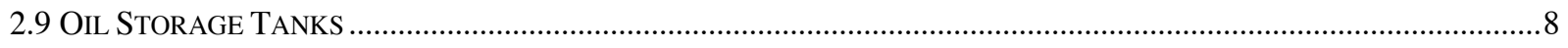

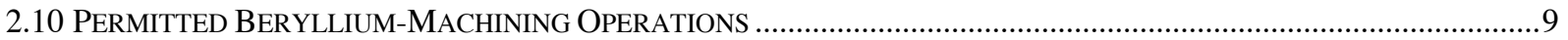

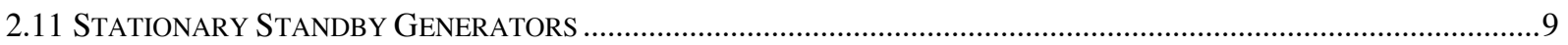

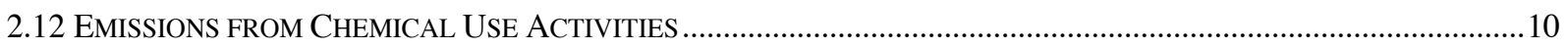

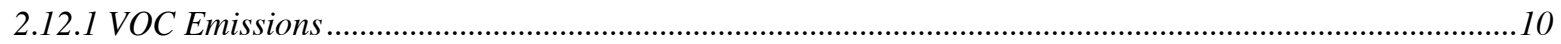

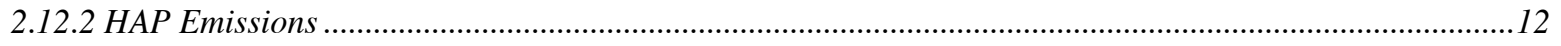

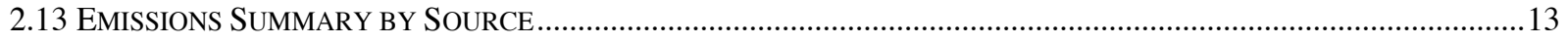

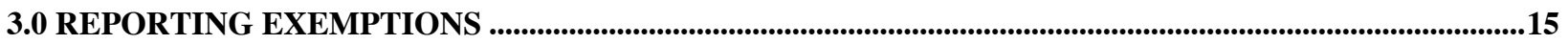

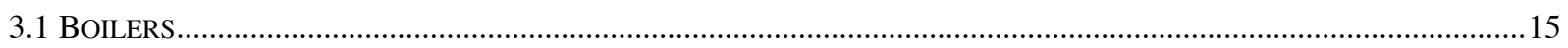

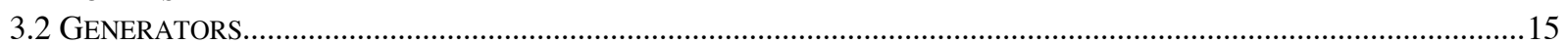

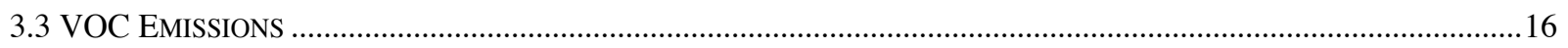

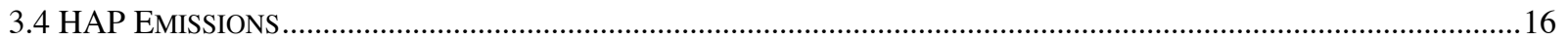

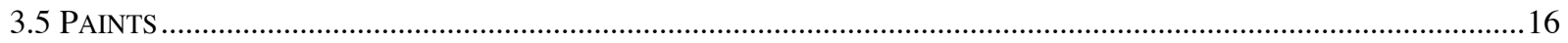

4.0 EMISSIONS SUMMARY …...............................................................................................................................................................18

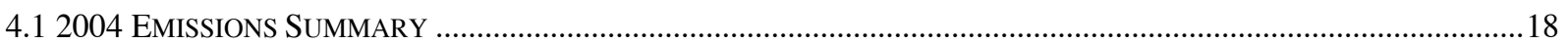

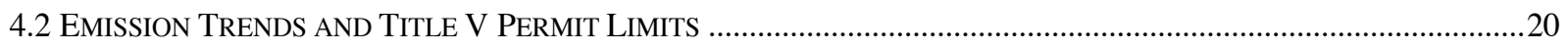

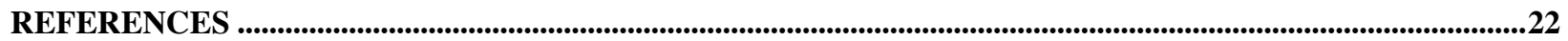

ATTACHMENT A. EMISSION CALCULATION WORKSHEETS FOR INDIVIDUAL EMISSION

UNITS.....................................................................................................................................................................................23

ATTACHMENT B. 2004 ANNUAL EMISSIONS INVENTORY SUBMITTAL TO NMED..................................47

ATTACHMENT C. 2004 SEMI-ANNUAL EMISSIONS REPORTS SUBMITTED UNDER TITLE V

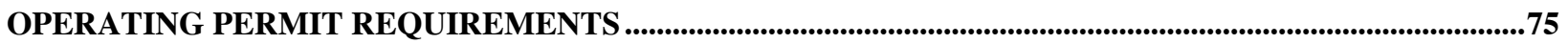




\section{LIST OF TABLES}

2-1. Sources Included in LANL's 2004 Annual Emissions Inventory and Semi-Annual Emissions Reports 5

2-2. Summary of LANL 2004 Reported Emissions for Annual Emissions Inventory ..... 14

2-3. Summary of LANL 2004 Semi-Annual Emissions as Reported Under Title V Operating Permit Requirements 14

3-1. Exemptions Applied for Chemical Use Activities 17

4-1. LANL Facility-Wide Criteria Pollutant Emissions for 2004 19

4-2. LANL HAP Emissions from Chemical Use for 2004 19

\section{LIST OF FIGURES}

2-1. Main steam plant at LANL TA-3 ........................................................... 6

2-2. Example of chemical use in laboratory hood at LANL ................................... 11

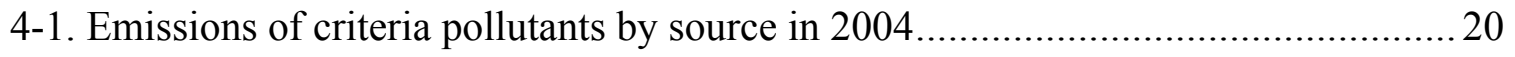

4-2. Comparison of facility-wide emissions from 1999-2004.................................... 21

4-3. VOC and HAP emissions from annual reported chemical use, 1999-2004 ............ 21 


\title{
Emissions Inventory Report Summary for Los Alamos National Laboratory for Calendar Year 2004
}

\author{
by
}

\author{
Meteorology and Air Quality Group (ENV-MAQ)
}

\begin{abstract}
Los Alamos National Laboratory (LANL) is subject to annual emissions reporting requirements for regulated air pollutants under Title 20 of the New Mexico Administrative Code, Chapter 2, Part 73 (20.2.73 NMAC), Notice of Intent and Emissions Inventory Requirements. The applicability of the requirements is based on the Laboratory's potential to emit 100 tons per year of suspended particulate matter, nitrogen oxides, carbon monoxide, sulfur oxides, or volatile organic compounds. Additionally, on April 30, 2004, LANL was issued a Title $\mathrm{V}$ Operating Permit from the New Mexico Environment Department, Air Quality Bureau, under 20.2.70 NMAC. This Title V Operating Permit (Permit No. P-100) includes emission limits and operating limits for all regulated sources of air pollution at LANL. The Title V Operating Permit also requires semi-annual emissions reporting for all sources included in the permit. This report summarizes both the annual emissions inventory reporting and the semi-annual emissions reporting for LANL for calendar year 2004. LANL's 2004 emissions are well below the emission limits in the Title V Operating Permit.
\end{abstract}

\subsection{INTRODUCTION}

\subsection{REgUlatory BASIS}

Los Alamos National Laboratory (LANL or the Laboratory) has reported on air pollutants generated from its operations since the 1970s when Air Quality Control Regulation 703, Registration of Air Contaminant Sources, was promulgated. According to the regulation, the Laboratory was required to register air pollutant sources that emitted more than 2,000 lb per year of any air contaminant. This regulatory requirement later evolved into Title 20 of the New Mexico Administrative Code, Chapter 2, Part 73 (20.2.73 NMAC), Notice of Intent and Emissions Inventory Requirements. The objective of the reporting requirement is to provide emissions data to the New Mexico Environment Department (NMED)/Air Quality Bureau (AQB) so its staff can determine whether LANL meets state and federal air pollutant standards. 
Annual emissions inventory reporting requirements under 20.2.73 NMAC apply to any stationary source which

- has been issued a construction permit under 20.2.72 NMAC;

- has been required to file a Notice of Intent under 20.2.73.200 NMAC; or

- emits in excess of

○ 1 ton per year of lead or

○ 10 tons per year of

- total suspended particulates;

- $\quad$ particulate matter $(\mathrm{PM})$ with diameter less than 10 micrometers $\left(\mathrm{PM}_{10}\right)$;

- $\mathrm{PM}$ with diameter less than 2.5 micrometers $\left(\mathrm{PM}_{2.5}\right)$;

- $\quad$ sulfur dioxide;

- $\quad$ nitrogen oxides $\left(\mathrm{NO}_{\mathrm{x}}\right)$;

- carbon monoxide (CO); or

- $\quad$ volatile organic compounds (VOCs).

The annual emissions inventory must be submitted to NMED/AQB by April 1 of each year. The NMED/AQB enters the data in the Aerometric Information Retrieval System (AIRS). ${ }^{1}$ This nationwide system, administered by the U.S. Environmental Protection Agency (EPA), is used to help ensure ambient air quality standards are maintained and to track the state's air pollutant emissions. AIRS is a large air pollution database that contains information, requirements, and data on air pollution and air quality in the United States and various World Health Organization member countries. The program is operated by the EPA and state/local air pollution control agencies. The AIRS database tracks each state's progress towards achieving and maintaining National Ambient Air Quality Standards (NAAQS) for criteria pollutants. The database is also used as a tool to help improve each state's air quality programs by enabling program members to access and compare past data and view data from other states. For 2004 emissions inventory reporting, NMED imported existing facility data from the AIRS database into spreadsheets and requested facilities to update the sheets with 2004 facility emissions information.

Additionally, on April 30, 2004, the Laboratory received their Title V Operating Permit (P-100) from the NMED/AQB ${ }^{2}$ as required under 20.2.70 NMAC. A condition of the Title V Operating Permit is that LANL must submit semi-annual emissions reports to NMED documenting that emissions from all permitted sources are below permitted emission levels. Section 4.0 of the permit states:

Reports of actual emissions from permitted sources in Section 2.0 of the permit shall be submitted on a 6 month basis. The reports shall include a comparison of actual emissions that occurred during the reporting period with the facility-wide allowable emission limits specified in Section 2.11 of the permit. The reports shall be submitted within 90 days from the end of the reporting period. The reporting periods are January 1 through June 30, and July 1 through December 31. This condition is pursuant to 20.2.70.302.E.1 NMAC. 
Therefore, for the first time, in 2004 the Laboratory submitted the semi-annual emissions reports as well as the annual emission inventory. There are a few differences in which sources are included in the two emissions reports. These differences are explained in the following sections.

\subsection{Contents of Annual Emissions inventory Submittal}

NMED requested that LANL submit annual emissions inventory data for 2004 via electronic format for entry into AIRS. The information required for submittal includes the following:

- company name, address, and physical location for the facility;

- facility contact information;

- signed certification statement by a responsible facility official; and

- specific information for each emission unit such as stack and exhaust parameters, type and efficiency of control equipment, schedule of operation, annual process or fuel combustion rates, and estimated actual emissions for 2004.

This annual emissions inventory submittal includes air pollutant data for $\mathrm{PM}, \mathrm{PM}_{10}, \mathrm{CO}, \mathrm{NO}_{\mathrm{x}}$, sulfur oxides $\left(\mathrm{SO}_{\mathrm{x}}\right)$, VOCs, beryllium, and aluminum. Additionally, at the request of NMED, the 2004 report provides data on emissions from hazardous air pollutants (HAPs), $\mathrm{PM}_{2.5}$, and ammonia.

The requirement to provide $\mathrm{PM}_{2.5}$ and ammonia emissions data stems from recent developments by EPA on a NAAQS for $\mathrm{PM}_{2.5}$. States are developing a baseline for $\mathrm{PM}_{2.5}$. As such, for the 2004 emissions inventory, NMED requested emissions information on $\mathrm{PM}_{2.5}$. Further, ammonia is a precursor to $\mathrm{PM}_{2.5}$ formation. It contributes to the secondary aerosol formation of $\mathrm{PM}_{2.5}$ by combining with $\mathrm{NO}_{\mathrm{x}}$ and $\mathrm{SO}_{\mathrm{x}}$ to form ammonium nitrate and fine sulfate particles. Therefore, NMED also requested emissions information on ammonia.

In the 2004 annual emissions inventory submittal, LANL provided $\mathrm{PM}_{2.5}$ emissions data for all combustion sources and other emission sources where $\mathrm{PM}_{2.5}$ emission factors were readily available. In the absence of $\mathrm{PM}_{2.5}$ emission factors, $\mathrm{PM}$ or $\mathrm{PM}_{10}$ emissions were assumed to be equivalent to $\mathrm{PM}_{2.5}$. The Laboratory does not operate any emission units that are sources of ammonia emissions. Ammonia was included in the facility-wide emission estimates for chemical use. 


\subsection{Contents of the Semi-Annual Title V Operating Permit Emissions Reports}

The semi-annual Title V Operating Permit emissions reports include actual emissions for the reporting period for each emission source or source category included in the Title V Operating Permit. For each source category, the actual emissions are compared to emission limits listed in the permit. The emissions are calculated using operating data from logbooks and records maintained on-site. All emission calculations are consistent with calculation methods used for the annual emissions inventory.

The semi-annual emissions report includes a few source categories not included in the annual emissions inventory. The Laboratory requested emission limits in their Title V Operating Permit for two source categories that are considered insignificant sources for the annual emissions inventory. These source categories are (1) small boilers and heaters and (2) stationary standby generators. LANL requested emission limits for these source categories to obtain federally enforceable limits that would keep the Laboratory under the major source threshold for Prevention of Significant Deterioration (PSD) applicability (20.2.74 NMAC). LANL's actual emissions from these insignificant sources have historically been very low, however, without federally enforceable limits on their operation, the potential to emit from these sources was quite high. To demonstrate that LANL is below the PSD applicability and is in compliance with the emission limits placed on these emission sources, LANL now must include these emissions in the semi-annual Title V Operating Permit emissions reports.

\subsection{REPORTED EMISSION SOURCES}

Table 2-1 shows the emission sources included in the Laboratory's 2004 Annual Emissions Inventory ${ }^{3}$ and the 2004 Semi-Annual Emissions reports. ${ }^{4}$ The source categories and the methodology used to calculate emissions are described in the following sections.

The following subsections describe emission sources included in the 2004 emissions inventory and semi-annual emissions reports and emission calculation methodology for each source type. A summary table of actual reported emissions by source is included at the end of this section (Section 2.13). Attachment A includes worksheets showing detailed emission calculations for individual emissions sources. A copy of the 2004 Emissions Inventory as submitted to NMED is presented in Attachment B. The semi-annual emissions reports are included as Attachment C.

\subsection{Steam Plants}

The Laboratory operates two steam plants, one located at TA-3 and the other at TA-21. The TA3 steam plant produces steam for heating and electricity for much of the Laboratory when sufficient power from outside sources is not available. The steam plant at TA-21 provides steam for heating of buildings at this technical area. The heat produced from both steam plants is used for comfort heat and hot water and to support facility processes. Each steam plant has three boilers that are fueled primarily with natural gas with diesel fuel as a backup. The TA-3 steam plant was originally included in LANL's 
Table 2-1. Sources Included in LANL's 2004 Annual Emissions Inventory and Semi-Annual Emissions Reports

\begin{tabular}{|c|c|c|}
\hline $\begin{array}{l}\text { Included in Annual } \\
\text { Emissions Inventory }\end{array}$ & $\begin{array}{l}\text { Included in Semi-Annual } \\
\text { Emissions Reports }\end{array}$ & Comment \\
\hline $\begin{array}{l}\text { Steam Plants (Technical } \\
\text { Area [TA] } 3 \text { \& TA-21) }\end{array}$ & $\begin{array}{l}\text { Steam Plants (TA-3 \& } \\
\text { TA-21) }\end{array}$ & \\
\hline $\begin{array}{l}\text { Boilers greater than } 5 \\
\text { MMBTU/hr (14 units) }\end{array}$ & $\begin{array}{l}\text { All small and large boilers } \\
\text { and heaters } \\
\text { (approximately } 175 \text { units) }\end{array}$ & $\begin{array}{l}\text { Small boilers less than } 5 \\
\text { MMBTU/hr are exempt from } \\
\text { annual emission inventory } \\
\text { requirements (see Section } 3.1 \text { ). }\end{array}$ \\
\hline Asphalt Plant & Asphalt Plant & \\
\hline $\begin{array}{l}\text { Paper Shredder and Data } \\
\text { Disintegrator }\end{array}$ & $\begin{array}{l}\text { Paper Shredder and Data } \\
\text { Disintegrator }\end{array}$ & \\
\hline Rock Crusher (shut down) & Rock Crusher (shut down) & \\
\hline Degreasers & Degreasers & \\
\hline $\begin{array}{l}\text { Air Curtain Destructors } \\
\text { (shut down) }\end{array}$ & Not included & $\begin{array}{l}\text { Air curtain destructors were } \\
\text { shut down before issuance of } \\
\text { the Title V Operating Permit. }\end{array}$ \\
\hline Carpenter Shops & Carpenter Shops & \\
\hline Oil Storage Tanks & No tanks included & $\begin{array}{l}\text { Applicability of the New } \\
\text { Source Performance Standard } \\
\text { for storage tanks changed in } \\
2004 \text { and the LANL oil storage } \\
\text { tanks were not required to be } \\
\text { included in the Title V } \\
\text { Operating Permit. }\end{array}$ \\
\hline $\begin{array}{l}\text { Permitted Beryllium } \\
\text { Sources }\end{array}$ & $\begin{array}{l}\text { Permitted Beryllium } \\
\text { Sources }\end{array}$ & \\
\hline Facility-wide Chemical Use & $\begin{array}{l}\text { Facility-wide Chemical } \\
\text { Use }\end{array}$ & \\
\hline $\begin{array}{l}\text { Process Generators (not yet } \\
\text { operational) }\end{array}$ & $\begin{array}{l}\text { Process Generators, and } \\
\text { Stationary standby } \\
\text { generators (approximately } \\
45 \text { units) }\end{array}$ & $\begin{array}{l}\text { Stationary standby generators } \\
\text { are exempt from annual } \\
\text { emission inventory } \\
\text { requirements (see Section } 3.2 \text { ). }\end{array}$ \\
\hline
\end{tabular}

emissions inventory as AIRS ID No. 002. When a modification to the plant was made in 2001, the TA-3 steam plant was separated into three AIRS ID numbers $(032,033$, and 034) for emissions reporting purposes. The three boilers at the TA-21 steam plant are included in the emissions inventory as one AIRS ID number (004).

Actual emissions are calculated on the basis of metered fuel consumption and emission factors. The primary source of emission factors is AP-42, the U.S. Environmental Protection Agency's Compilation of Air Pollutant Emission Factors. ${ }^{5}$ However, emission factors from stack tests conducted at the TA-3 steam plant when burning natural gas were also used, as appropriate.

The TA-3 steam plant has historically been the largest source of $\mathrm{NO}_{\mathrm{x}}$ emissions at the Laboratory. In 2002 a voluntary project to install pollution control equipment on the three boilers 
at the TA-3 steam plant was completed. The three boilers were fitted with flue gas recirculation (FGR) equipment to reduce $\mathrm{NO}_{\mathrm{x}}$ emissions. Stack testing for $\mathrm{NO}_{\mathrm{x}}$ and $\mathrm{CO}$ was conducted before FGR equipment was installed and again after it was operational. Based on these stack test results, FGR reduced $\mathrm{NO}_{x}$ emissions by approximately 64 percent. The FGR equipment was operational for all of 2004. Figure 2-1 shows a picture of the TA-3 steam plant building and stacks.

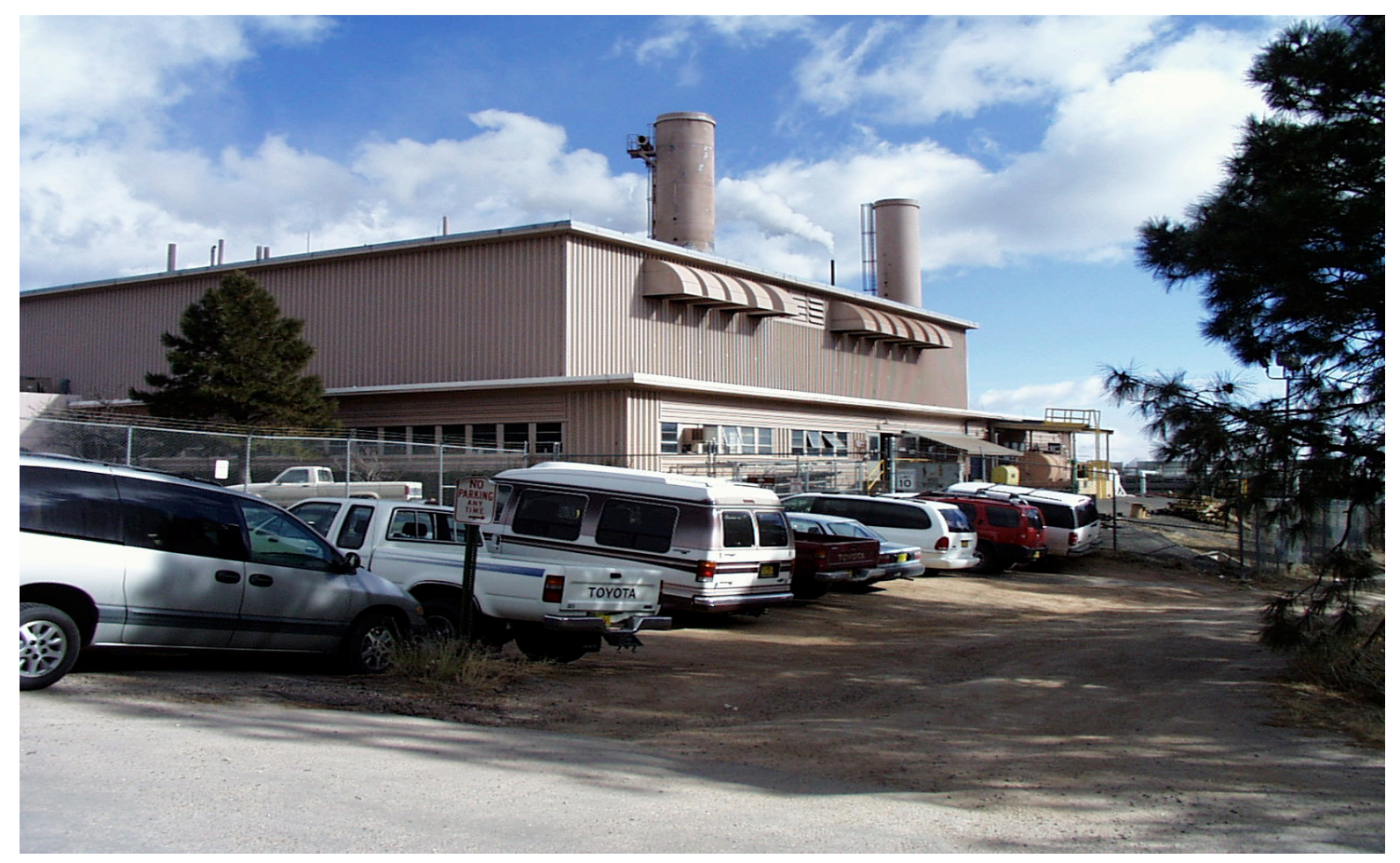

Figure 2-1. Main steam plant at LANL TA-3.

\subsection{SMall BoIlers AND Heaters}

The Laboratory operates approximately 200 small boilers and heaters, used primarily for seasonal comfort heat. Most of the boilers are exempt from permitting requirements because of their small size and use as comfort boilers and are not included in the annual emissions inventory. The exemption analysis applied to boilers is discussed in Section 3.1 of this report.

The nonexempt boilers reported in the 2004 annual emissions inventory include the following:

- four boilers at TA-16 (AIRS ID Nos. 024 and 025);

- three boilers at TA-48 (AIRS ID Nos. 015, 016, and 017);

- two boilers at TA-53 (AIRS ID Nos. 018 and 019);

- two boilers at TA-59 (AIRS ID Nos. 020 and 021);

- two boilers at TA-55 (AIRS ID Nos. 037 and 038); and

- one process-related boiler at TA-50 (AIRS ID No. 044).

All of the reported boilers burn natural gas. Operating logs for the TA-55 and TA-50 boilers were used to quantify fuel use for these units. Fuel use for all other boilers was estimated based on the total amount of natural gas used by the Laboratory minus the amount supplied to metered sources. The amount of natural gas left after subtracting out metered sources was apportioned to 
the various boilers based on their size. Since they are all seasonal boilers used for building heating, it was assumed they would all operate approximately the same amount of time over the course of the year. Some emission factors were available from stack tests (TA-55), some were provided by the boiler manufacturer (Sellers Engineering Company), and the rest were taken from AP-42. ${ }^{5}$ Copies of spreadsheets showing fuel use and emission factors for each boiler are included in Attachment A.

For the semi-annual emissions reports, emissions from all small boilers and heaters are included as a source category. The Title V Operating Permit includes emissions limits for this group of emission sources. To estimate emissions all un-metered fuel use was multiplied by AP-42 emission factors for small boilers burning natural gas. ${ }^{5}$ Total emissions of each pollutant from all boilers and heaters in this source category were then summed and reported on the semi-annual emissions reports.

\subsection{Asphalt Plant}

For over 40 years LANL operated an asphalt plant to produce small amounts of asphalt for road repairs in and around the Laboratory. This unit is included in LANL's emissions inventory as AIRS ID No. 013. In June 2003 the asphalt plant was shut down in anticipation of a new asphalt plant being built. In September 2003 the old asphalt plant was dismantled and removed. The new asphalt plant will be located at TA-60; however, construction of the new plant has been delayed due to nesting of spotted owls. The new asphalt plant was not operational during 2004. When the new asphalt plant begins operation an AIRS ID number will be assigned and emissions will be reported in the annual emission inventory and the semi-annual emissions reports.

\subsection{PAPer Shredder/DAta Disintegrator}

Shredding operations from the paper shredder at TA-52 are a source of PM emissions. This unit is included in LANL's emissions inventory as AIRS ID No. 027. Estimates of actual emissions are based on the number of boxes of material shredded and engineering estimates for controlled emissions. The unit is equipped with a cyclone separator and baghouse for abatement. The emission factor, which is the amount of particulate estimated to enter into the exhaust, was provided by the shredder equipment manufacturer. The air abatement system efficiencies were estimated based on information from EPA on cyclone and baghouse efficiencies and are documented in LANL's Title V Operating Permit application submitted to NMED in November 2002. ${ }^{6}$

In July 2004 this paper shredder was taken out of service and replaced with a new data disintegrator to shred paper and other media. The new data disintegrator is included in the emissions inventory at AIRS ID No. 045. Emissions are estimated in much the same way as described for the old paper shredder. For 2004 emissions from the old paper shredder are based on operating logs from January through June. Emissions from the new data disintegrator are calculated from operating logs from July through December.

These two sources are reported in both the annual emission inventory and the semi-annual emissions report. 


\subsection{ROCK CRUSHER}

In June 1999, NMED issued a 20.2.72 NMAC construction permit (Permit No. 2195) to LANL to operate an impact rock crusher to crush potentially radioactive contaminated concrete removed from buildings as part of the Laboratory's decontamination and decommissioning efforts. This unit is included in LANL's emissions inventory as AIRS ID No. 026. The rock crusher was not operated in 2004, and the permit was retired on June 10, 2004.

\subsection{DEGREASERS}

The halogenated solvent cleaning machine at TA-55 has a capacity of 18 liters and is registered with NMED/AQB as required under the National Emissions Standards for Hazardous Air Pollutants, 40 CFR 63 Subpart T, "Halogenated Solvent Cleaning." The solvent used in the machine, trichloroethylene (Chemical Abstracts Service [CAS] No. 79-01-6), is a VOC and a HAP. This emission unit is included in the annual emissions inventory as AIRS ID No. 028. LANL uses a mass balance approach to estimate emissions. Logbooks are kept on the amount of solvent added and removed from the machine. Additionally, monthly tracking of solvent levels in the machine are logged. Using a mass balance approach, emissions are estimated. LANL has two additional halogenated solvent cleaning machines registered with NMED (AIRS ID Nos. 029 and 030); however, these two machines did not operate in 2004. This source category is reported in both the annual emissions inventory and the semi-annual emissions report.

\subsection{Air Curtain Destructors}

Three air curtain destructors were used extensively during the period from 2001 through 2003 for controlled open burning of wood and brush generated from tree thinning activities at LANL. These units are included in LANL's emissions inventory as AIRS ID Nos. 039, 040, and 041. These units were retired and taken out of service in October 2003 and did not operate during 2004.

\subsection{CARPENTER SHOP}

LANL operates a carpenter shop at TA-3-38. This carpenter shop was built before 1960 and is not subject to 20.2.72 NMAC construction permitting. However, LANL included carpenter shops in the Title V Operating Permit. Therefore, this source category is included in the annual emissions inventory as AIRS ID No. 042 and is included on the semi-annual emissions report. Additionally, a carpenter shop located at TA-15 is included in the Operating Permit. However, this carpenter shop is still under construction and operations have not started up yet. When this carpenter shop is operational, emissions will be included in both the annual emissions inventory and the semi-annual emissions reports.

PM emissions from the carpenter shop are estimated based on number of hours the exhaust system operated and an estimated control efficiency of the cyclone. Emission factors from AP42, Section 10.4, "Woodworking Waste Collection Operations," were used to estimate PM, $\mathrm{PM}_{10}$, and $\mathrm{PM}_{2.5}$ emissions. 7

\subsection{Oil Storage Tanks}

Two large diesel storage tanks are located at the TA-3 steam plant for backup fuel to the boilers. These tanks are included in the annual emissions inventory as AIRS ID Nos. 035 and 036. 
Emissions from these tanks are estimated using software developed by EPA for estimating emissions from storage tanks. ${ }^{8}$ The TANKS 4.0 software requires inputs for tank parameters, site-specific meteorological conditions, and actual fuel throughputs.

The Laboratory included 14 smaller oil storage tanks in the November 2002 updated Title V Operating Permit application because they were subject to New Source Performance Standards, 40 CFR 60, Subpart Kb. These tanks store mineral oil, scintillation oil, or dielectric oil, which all have vapor pressures less than $0.01 \mathrm{mmHg}$. In 2003 EPA modified the applicability of Subpart $\mathrm{Kb}$ and these tanks are no longer subject to this regulation. Subsequently they were not included in the Laboratory's Title V Operating Permit (Permit No. P-100), and therefore are not included in the semi-annual emissions reports.

Emissions from these smaller oil storage tanks were included for the first time in the 2002 annual emissions inventory. With agreement from NMED, emissions from the 14 tanks were summed and listed as one stack entry in the emissions inventory report due to the small quantity of emissions (email correspondence with Jim Shively, NMED/AQB, dated February 3, 2003). This "composite" mineral oil tank was assigned AIRS ID No. 043. Because an AIRS ID number is now assigned, emissions from these tanks will continue to be included in the annual emissions inventory submittal. Based on the most conservative tank parameters and actual throughput from chemical inventory records, a unit emission rate was calculated. The TANKS 4.0 software was used to estimate emissions for both vertical and fixed roof tanks. ${ }^{8}$ Unit emission rates in $\mathrm{lb} / \mathrm{yr}$ were multiplied by the number of horizontal and vertical tanks to provide an estimate of total annual emissions from all of the tanks.

\subsection{Permitted Beryllium-Machining Operations}

The Laboratory operates under five 20.2.72 NMAC construction permits** for berylliummachining operations that are subject to 40 CFR 61, Subpart C, "National Emission Standards for Beryllium." Beryllium-machining operations are reported in the emissions inventory under AIRS ID Nos. 006, 007, 008, 010, and 011. AIRS ID No 008 for beryllium machining at TA-3, Building 102 was not operated in 2004, and the permit was retired on February 20, 2004. Emissions reported for the Beryllium Test Facility (AIRS ID No. 007) are from actual stack emissions measurements. Emissions for the Target Fabrication Facility (AIRS ID No. 006) are from initial compliance stack testing. Emissions from the plutonium facility (AIRS ID Nos. 010 and 011) are reported at permitted emission levels. Total emissions from all permitted beryllium operations are included in the semi-annual emissions reports.

\subsection{STATIONARY STANDBY GENERATORS}

The Laboratory received a Construction Permit in October 2002 to install a process-related generator at TA-33. Due to a series of delays, this generator is not yet operational. The TA-33 generator is included in LANL's Title V Operating Permit. When this generator becomes operational emissions will be included in both the annual emissions inventory and the semiannual emissions reports.

The Laboratory maintains approximately 45 stationary standby generators that are considered exempt sources under the Construction Permit regulations (20.2.72.202.b NMAC) and the annual

\footnotetext{
*** Permit No. 632, issued December 26, 1985; Permit No. 632-M2, issued October 30, 1998; Permit No. 635, issued March 19, 1986; Permit No. 636, issued March 19, 1986; Permit No. 1080-M1-R2, issued March 11, 1998.
} 
emission inventory requirements. However, these sources are included in LANL's Title V Operating Permit with operating limits and emission limits. Therefore these sources must be included in the semi-annual emissions reports. All stationary standby generators at LANL are exercised on a routine schedule to ensure they are operational and will function properly if needed. All units are equipped with hour meters to document how many hours they are used. The Laboratory maintains records on a semi-annual basis to document hour meter readings. The number of hours each generator is used in a reporting period is multiplied by AP-42 emission factors for diesel-fired internal combustion engines, or natural gas-fired internal combustion engines. ${ }^{9}$ Emissions are then summed for each pollutant and reported on the semi-annual emissions reports for this source category.

\subsection{EMISSIONS FROM CHEMICAL USE ACTIVITIES}

The majority of the Laboratory's work is devoted to research and development (R\&D) activities. Varying operating parameters, as well as amounts and types of chemicals, are used in these activities. R\&D activities occur at virtually all technical areas within the Laboratory, typically in small quantities in laboratory settings. Figure 2-2 shows a typical laboratory at LANL where chemicals are used.

For the purposes of annual emissions inventory reporting, one AIRS ID has been assigned for all R\&D chemical use (AIRS ID No. 031). Facility-wide chemical use emissions are reported on both the annual emissions inventory and the semi-annual emissions report. The methods used to quantify emissions of VOC and HAPs from R\&D activities are discussed below.

\subsubsection{VOC Emissions}

The Laboratory tracks chemical purchases through a facility-wide chemical tracking system called ChemLog. A download from the ChemLog inventory system was created that included all chemical containers added to LANL's inventory between January 1, 2004, and December 31, 2004. This dataset included 37,179 separate line items of chemicals purchased. The volume of chemicals purchased is approximately 15 percent lower than previous years due to the Laboratory-wide shutdown in July 2004 when the Laboratory stopped operations to focus on evaluating and updating safety and security measures. 


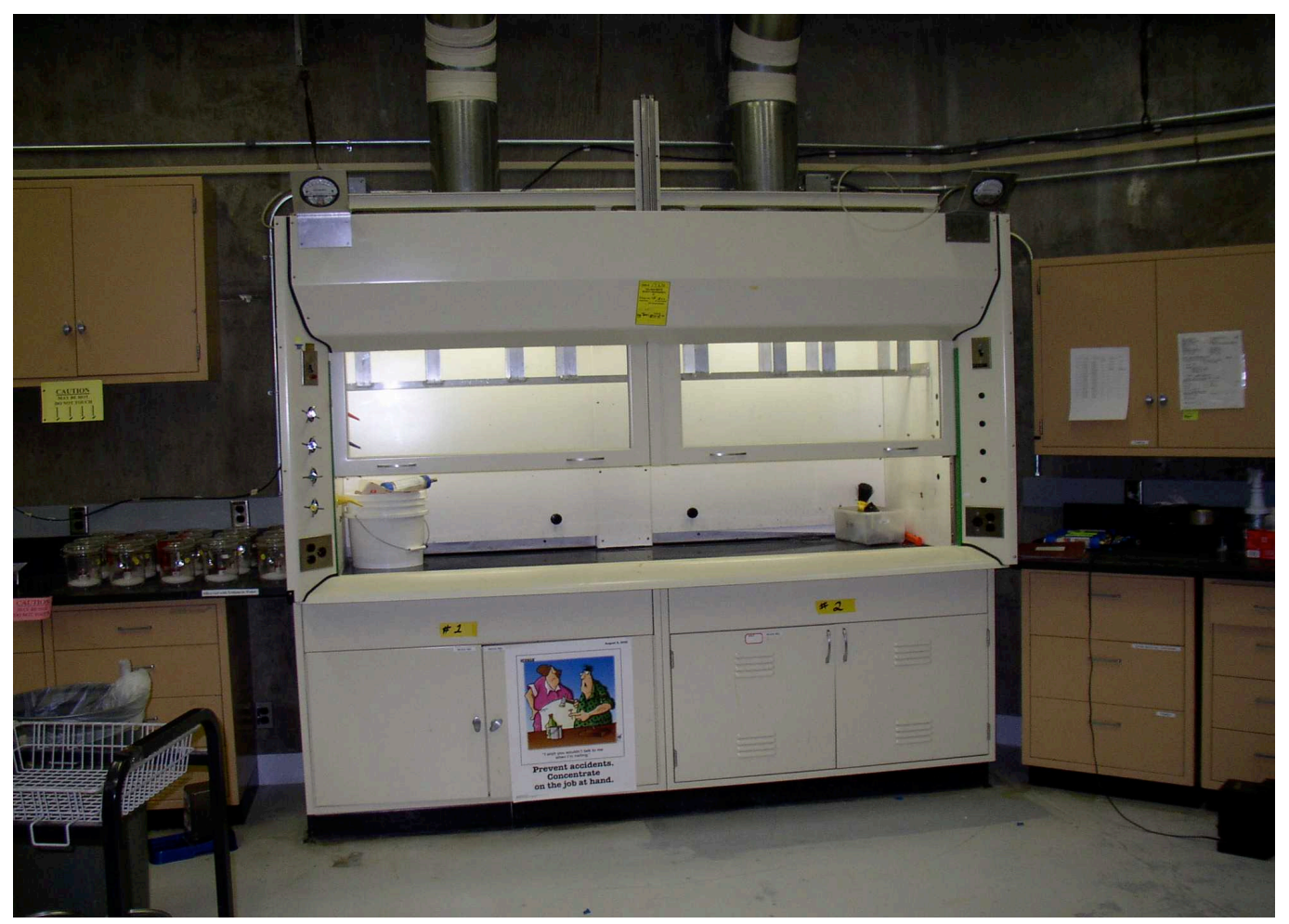

Figure 2-2. Example of chemical use in laboratory hood at LANL.

The dataset was reviewed electronically to identify all VOCs purchased and received at LANL in 2004. With the exception of specific listed chemicals, VOCs are any compounds of carbon that participate in atmospheric photochemical reactions. VOCs include commonly used chemicals such as ethanol, methanol, trichloroethylene, and isopropanol. The general assumption used in estimating VOC emissions from chemical use is

$$
\text { Purchasing }=\text { Use }=\text { Emissions. }
$$

From the dataset of chemicals purchased in 2004, certain categories of chemicals were separated and eliminated from the analysis. The classifications assigned and corresponding reasons (noted in parentheses) for exclusion of chemicals from inventory records are noted below.

- Solid materials (not a significant source of air emissions based on their low vapor pressure);

- Non-VOC materials as defined by 40 CFR 51.100 (specific chemicals in 40 CFR 51.100 are listed as having negligible photochemical reactivity and are exempt from the definition of VOC);

- Paints (paints were evaluated separately_see Section 3.5);

- Inorganic chemicals (inorganics are not compounds of carbon);

- Oils (not a significant source of air emissions based on low vapor pressure and primarily used for maintenance);

- Fuels used for combustion purposes (emissions from fuel combustion are reported for each combustion unit).

Furthermore, the following categories of chemicals were eliminated based on guidance from NMED (letter from Mary Uhl, NMED/AQB, dated January 30, 2001): 
- Container sizes of $1 \mathrm{lb}$ or less;

- Chemicals with vapor pressures less than $10 \mathrm{mmHg}$;

- Chemicals used to calibrate equipment;

- Maintenance chemicals;

- Use of office equipment and products;

- Chemicals used for boiler water treatment operations;

- Chemicals used for oxygen scavenging (deaeration) of water; and

- Chemicals used in bench-scale chemical analysis.

After elimination of chemicals and categories of chemicals listed above, the remaining chemical inventory records were matched with a list of known VOCs by CAS number. For mixtures (chemicals without CAS numbers) material safety data sheets (MSDSs) were reviewed to determine if any VOCs were present and, if so, to determine the associated percent volatile. As a conservative estimate, VOCs identified in ChemLog records were assumed to be 100 percent emitted to air. Estimated emissions of VOCs from chemical use in 2004 totaled 7.95 tons.

\subsubsection{HAP Emissions}

Section 112(b) of the 1990 Clean Air Act Amendments listed 189 unique HAPs identified for potential regulation by EPA. In 1995, caprolactam was delisted as a HAP. Of the remaining 188 listed HAPs, 17 are classes of compounds (e.g., nickel compounds). Use of the 188 listed chemicals in activities at the Laboratory was evaluated and quantified for the annual emissions inventory submittal to NMED.

The ChemLog inventory system 2004 data set was analyzed to identify HAPs. The identification process was similar to that used for VOCs. Pure chemicals (i.e., chemicals with CAS numbers), classes of compounds, and mixtures were evaluated to determine if the chemicals themselves were HAPs or if they contained HAP constituents. For mixtures, MSDSs were reviewed to determine if any HAPs were present and, if so, to determine the associated HAP percentages. Listed below are certain chemical types or categories that were identified and removed from this analysis (refer to Section 2.12.1 and Table 3-1 for explanations on removal of these chemicals):

- Paints;

- Oils;

- Maintenance chemicals;

- Chemicals used to calibrate equipment;

- Container sizes of $1 \mathrm{lb}$ or less;

- Chemicals used in bench-scale chemical analysis;

- Use of office equipment and products;

- Chemicals used for boiler water treatment operations; and

- Chemicals used for oxygen scavenging (deaeration) of water.

Total HAP emissions were estimated by summing (1) pure HAP chemicals, (2) classes of compounds that are HAPs, and (3) the HAP constituents from mixtures. The resulting total amount of HAPs from chemical use reported for 2004 was 5.71 tons.

\footnotetext{
* This exemption was applied only to biological research solutions. Otherwise, this exemption was not applied. See Table 3-1.
} 
The HAP emissions reported generally reflect quantities procured in the calendar year. In a few cases procurement values and operational processes were further evaluated so that actual air emissions could be reported instead of procurement quantities. Additional analyses for certain metals and acids were performed and are described below.

\section{HAP Metals}

Purchases of chromium, lead, manganese, mercury, and nickel compounds were evaluated to determine usage and potential air emissions. Several of the purchases were identified as laboratory calibration standards containing only parts per million quantities of the metals. These were exempt from emissions inventory requirements because of their use as standards for calibrating laboratory equipment. Other purchasers of relatively large quantities of metal compounds that were contacted confirmed that the material was still in use or in storage and had not resulted in air emissions.

\section{Hydrochloric Acid}

Facility and Waste Operations Division purchased multiple 14-gallon carboys of hydrochloric acid $(\mathrm{HCl})$ totaling approximately 1,592 lb. This $\mathrm{HCl}$ was used for heat exchanger scale cleaning and for cleaning of electrodialysis reversal membranes. Emissions from these particular activities were estimated to be less than one pound based on specific process information and engineering calculations. This is also considered a routine maintenance activity and exempt from emissions inventory reporting. The remaining procurements consisted of numerous small purchases from a variety of operating groups. Additional analysis of these numerous small purchases was not done. As a conservative assumption, all of this $\mathrm{HCl}$ was assumed to be emitted resulting in a reported total of 1.52 tons of $\mathrm{HCl}$ emissions.

\subsection{Emissions Summary by Source}

Table 2-2 provides a summary of LANL's 2004 actual emissions, as submitted for the annual emissions inventory. The table presents emissions by pollutant and by source, with a facility total at the bottom of the table. Attachment A provides detailed information on how emissions were calculated for each emission unit. 
Table 2-2. Summary of LANL 2004 Reported Emissions for Annual Emissions Inventory

\begin{tabular}{|c|c|c|c|c|c|c|c|}
\hline & $\begin{array}{c}\mathrm{NO}_{\mathbf{X}} \\
(\text { tons/yr) }\end{array}$ & $\begin{array}{c}\mathrm{SO}_{\mathrm{x}} \\
\text { (tons/yr) } \\
\end{array}$ & $\begin{array}{c}\mathbf{P M}_{10} \\
\text { (tons/yr) }\end{array}$ & $\begin{array}{c}\mathbf{P M}_{2.5} \\
\text { (tons/yr) }\end{array}$ & $\begin{array}{c}\mathrm{CO} \\
\text { (tons/yr) } \\
\end{array}$ & $\begin{array}{c}\text { VOC } \\
\text { (tons/yr) }\end{array}$ & $\begin{array}{c}\text { HAPs } \\
\text { (tons/yr) }\end{array}$ \\
\hline $\begin{array}{l}\text { TA-3 Steam Plant } \\
\text { Boilers }\end{array}$ & 16.34 & 0.29 & 2.16 & 2.15 & 11.26 & 1.54 & 0.53 \\
\hline $\begin{array}{l}\text { TA-21 Steam Plant } \\
\text { Boilers }\end{array}$ & 1.58 & 0.01 & 0.12 & 0.12 & 1.33 & 0.09 & 0.03 \\
\hline Non-Exempt Boilers & 6.55 & 0.04 & 0.61 & 0.61 & 4.5 & 0.38 & 0.13 \\
\hline Asphalt Plant ${ }^{(\mathrm{a})}$ & 0.0 & 0.0 & 0.0 & 0.0 & 0.0 & 0.0 & 0.0 \\
\hline $\begin{array}{l}\text { Paper Shredder/Data } \\
\text { Disintegrator }\end{array}$ & $\mathrm{NA}^{*}$ & NA & 0.09 & 0.06 & NA & NA & NA \\
\hline Rock Crusher $^{(a)}$ & 0.0 & 0.0 & 0.0 & 0.0 & 0.0 & 0.0 & 0.0 \\
\hline Degreaser & $\mathrm{NA}$ & $\mathrm{NA}$ & $\mathrm{NA}$ & NA & NA & 0.01 & 0.01 \\
\hline $\begin{array}{l}\text { Air Curtain } \\
\text { Destructors }^{(a)}\end{array}$ & 0.0 & 0.0 & 0.0 & 0.0 & 0.0 & 0.0 & 0.0 \\
\hline Carpenter Shop & NA & NA & 0.02 & 0.01 & NA & NA & NA \\
\hline Oil Storage Tanks & NA & NA & NA & NA & NA & 0.05 & NA \\
\hline R\&D Chemical Use & NA & NA & NA & NA & NA & 7.95 & 5.71 \\
\hline TOTAL & 24.47 & 0.34 & 3.0 & 2.95 & 17.09 & 10.02 & 6.41 \\
\hline
\end{tabular}

*Not applicable.

Table 2-3 provides a summary of 2004 emissions as reported on the semi-annual emissions reports required by the Title V Operating Permit. Attachment A provides detailed information on how emissions were calculated for each emission source category.

Table 2-3. Summary of LANL 2004 Semi-Annual Emissions as Reported Under Title V Operating Permit Requirements

\begin{tabular}{|c|c|c|c|c|c|c|c|}
\hline & $\begin{array}{c}\mathrm{NO}_{\mathbf{X}} \\
(\text { tons/yr) }\end{array}$ & $\begin{array}{c}\mathrm{SO}_{\mathrm{x}} \\
(\mathrm{tons} / \mathrm{yr})\end{array}$ & $\begin{array}{c}\mathbf{P M}_{10} \\
\text { (tons/yr) }\end{array}$ & $\begin{array}{c}\mathbf{P M}_{2.5} \\
\text { (tons/yr) }\end{array}$ & $\begin{array}{c}\mathrm{CO} \\
(\text { tons/yr) }\end{array}$ & $\begin{array}{c}\text { VOC } \\
\text { (tons/yr) }\end{array}$ & $\begin{array}{c}\text { HAPs } \\
\text { (tons/yr) }\end{array}$ \\
\hline $\begin{array}{l}\text { TA-3 Steam Plant } \\
\text { Boilers }\end{array}$ & 16.34 & 0.29 & 2.16 & 2.15 & 11.26 & 1.54 & 0.53 \\
\hline $\begin{array}{l}\text { TA-21 Steam Plant } \\
\text { Boilers }\end{array}$ & \multicolumn{7}{|c|}{ Emissions included in Small Boilers Source Category } \\
\hline $\begin{array}{l}\text { All Small Boilers and } \\
\text { Heaters }\end{array}$ & 28.30 & 0.18 & 2.23 & 2.23 & 22.75 & 1.61 & 0.50 \\
\hline Asphalt Plant ${ }^{(a)}$ & 0.0 & 0.0 & 0.0 & 0.0 & 0.0 & 0.0 & 0.0 \\
\hline $\begin{array}{l}\text { Paper Shredder/Data } \\
\text { Disintegrator }\end{array}$ & NA* & NA & 0.09 & 0.06 & NA & NA & NA \\
\hline Rock Crusher $^{\text {(a) }}$ & 0.0 & 0.0 & 0.0 & 0.0 & 0.0 & 0.0 & 0.0 \\
\hline Degreaser & $\mathrm{NA}$ & NA & NA & NA & NA & 0.01 & 0.01 \\
\hline $\begin{array}{l}\text { Air Curtain } \\
\text { Destructors }^{(a)(b)}\end{array}$ & 0.0 & 0.0 & 0.0 & 0.0 & 0.0 & 0.0 & 0.0 \\
\hline Carpenter Shop & NA & NA & 0.02 & 0.01 & NA & NA & NA \\
\hline Oil Storage Tanks ${ }^{(b)}$ & $\mathrm{NA}$ & NA & NA & NA & NA & $\mathrm{NA}$ & NA \\
\hline R\&D Chemical Use & NA & NA & NA & NA & NA & 7.95 & 5.71 \\
\hline $\begin{array}{l}\text { Stationary Standby } \\
\text { Generators }\end{array}$ & 5.87 & 1.10 & 0.3 & 0.3 & 1.36 & 0.32 & 0.003 \\
\hline TOTAL & 50.5 & 1.57 & 4.8 & 4.75 & 35.4 & 11.4 & 6.75 \\
\hline
\end{tabular}

* NA = Not Applicable

(a) Source did not operate in 2004.

(b) Source category not included in Title V Operating Permit. 


\subsection{REPORTING EXEMPTIONS}

Specific activities that are determined to be insignificant under NMED's Operating Permit program (20.2.70 NMAC) are exempt from reporting under the emissions inventory requirements (20.2.73.300 NMAC). NMED has designated exempt sources, activities, or thresholds in the following lists:

- "List of Insignificant Activities," September 29, 1995, ${ }^{10}$ and

- "List of Trivial Activities," January 10, 1996. ${ }^{11}$

Laboratory sources and activities that qualify as insignificant or trivial as specified in these lists are not included in the annual emissions inventory. The following subsections of this report provide information and examples of the Laboratory's exempt activities as well as analyses performed to determine exempt status.

\subsection{BOILERS}

The Laboratory's boiler inventory was evaluated against the "List of Insignificant Activities." Specifically, boilers were exempted from emissions inventory reporting requirements if they met one of the following requirements:

- Fuel burning equipment which uses gaseous fuel, has a design rate less than or equal to five (5) million BTU per hour, and is used solely for heating buildings for personal comfort or for producing hot water for personal use, or

- Any emissions unit...that has the potential to emit no more than one (1) ton per year of any regulated pollutant...

Any boiler that was not used exclusively for comfort heating or hot water was evaluated for the one (1) ton per year exemption. For purposes of determining exemptions, boiler design ratings were used to estimate potential to emit. Any boiler not qualifying for one of these two exemptions is included in the annual emissions inventory with its own unique AIRS ID number.

For the semi-annual emissions reports, emissions from all boilers and heaters were summed and reported for the entire source category.

\subsection{GENERATORS}

The Laboratory maintains an inventory of approximately 125 portable generators. Portable generators are used at the Laboratory for temporary operations requiring remote power or to provide emergency backup power during power outages at various sites. The portable generators are fueled by gasoline and/or diesel fuel.

In addition to portable generators, the Laboratory maintains and operates approximately 45 stationary standby generators. Stationary generators are used on standby (emergency) status to provide power to critical systems at the Laboratory during power outages. The stationary generators are fueled by natural gas, gasoline, or diesel.

The insignificant activity exemptions applicable to the Laboratory's generators are the following:

- Portable engines and portable turbines that have a design capacity...less than or equal to 
○ 200-horsepower engine if fueled by diesel or natural gas, and

○ 500-horsepower engine if fueled by gasoline.

- Emergency generators that comply with the definition of standby equipment.

Standby equipment is defined in NMED/AQB "List of Insignificant Activities"10 as

"an emissions unit which on a temporary basis replaces equipment used in normal operation, and which either has an allowable emission rate or potential to emit for each fee pollutant that is equal to or less than the equipment replaced, or which does not operate for a period exceeding 500 hours per calendar year."

On the basis of size, portable generators used for temporary power at remote locations are exempt from emissions inventory reporting requirements. Further, LANL's small portable generators are considered trivial activities and are not included in the Title V Operating Permit or semi-annual emissions reports. All stationary generators are designated as standby equipment under the Operating Permit Program and are used solely to provide emergency backup power for less than 500 hours per year. Therefore they are considered insignificant sources and are also exempt from annual emissions inventory reporting requirements. However the stationary standby generators were voluntarily included as a source category in the Title V Operating Permit and are included in the semi-annual emissions reports.

The Laboratory is installing a process-related generator at TA-33 to support research activities. NMED issued a construction permit in October 2002 for installation of this generator (Permit No. 2195-F), and this unit is included in LANL's Title V Operating Permit. However, installation is not yet complete and the generator did not operate in 2004. Therefore, this unit is not included in the 2004 emissions inventory.

\subsection{VOC EMISSIONS}

A number of insignificant and trivial activities were applicable for exempting materials from the VOC chemical use total in the emissions inventory. The basis of the exemptions and corresponding insignificant or trivial activities are explained in Table 3-1.

Fuels such as propane, kerosene, and acetylene were analyzed separately and are not listed in Table 3-1. When fuels are burned in an open flame, almost all of the fuels are consumed and emissions are minimal. Emissions from fuel combustion are accounted for using emission factors for each fuel-burning unit.

\subsection{HAP EMISSIONS}

The HAP chemical use exemption analysis, similar to the VOC chemical use exemption analysis, resulted in application of several of the same exemptions from NMED/AQB "List of Trivial Activities" 10 and "List of Insignificant Activities"11 (refer to Table 3.1).

\subsection{PAInTS}

An analysis of VOC and HAP emissions resulting from painting activities conducted at the Laboratory was performed to determine if certain exemptions apply. Paint information for 2004 was gathered from work control databases and the ChemLog chemical inventory system. These records were evaluated for applicability of exemptions for trivial and insignificant activities. 
Table 3-1. Exemptions Applied for Chemical Use Activities

\begin{tabular}{|c|c|c|}
\hline $\begin{array}{c}\text { Basis of } \\
\text { Exemption }\end{array}$ & Activity Type & Activity \\
\hline $\begin{array}{l}\text { Container sizes of } \\
1 \text { pound or less }\end{array}$ & Trivial & $\begin{array}{l}\text { Paint or nonpaint materials dispensed from prepackaged } \\
\text { aerosol cans of } 16-\mathrm{oz} \text {. capacity or less. }\end{array}$ \\
\hline $\begin{array}{l}\text { Chemicals with } \\
\text { vapor pressures } \\
\text { less than } 10 \mathrm{mmHg}\end{array}$ & Insignificant & $\begin{array}{l}\text { Any emissions unit, operation, or activity that handles or } \\
\text { stores a liquid with vapor pressure less than } 10 \mathrm{mmHg} \text { or } \\
\text { in quantities less than } 500 \text { gal. }\end{array}$ \\
\hline $\begin{array}{l}\text { Calibration } \\
\text { chemicals }\end{array}$ & Trivial & $\begin{array}{l}\text { Routine calibration and maintenance of laboratory } \\
\text { equipment or other analytical instruments, including } \\
\text { gases used as part of those processes. }\end{array}$ \\
\hline $\begin{array}{l}\text { Maintenance } \\
\text { chemicals and oils }\end{array}$ & Trivial & $\begin{array}{l}\text { Activities that occur strictly for maintenance of grounds } \\
\text { or buildings, including lawn care; pest control; grinding; } \\
\text { cutting; welding; painting; woodworking; sweeping; } \\
\text { general repairs; janitorial activities; plumbing; re-tarring } \\
\text { roofs; installing insulation; steam-cleaning and water- } \\
\text { washing activities; and paving of roads, parking lots, and } \\
\text { other areas. } \\
\text { Activities for maintenance and repair of equipment, } \\
\text { pollution-control equipment, or motor vehicles either } \\
\text { inside or outside of a building. }\end{array}$ \\
\hline $\begin{array}{l}\text { Use of office } \\
\text { equipment and } \\
\text { products }\end{array}$ & Trivial & $\begin{array}{l}\text { Use of office equipment and products, not including } \\
\text { printers or businesses primarily involved in photographic } \\
\text { reproduction. }\end{array}$ \\
\hline $\begin{array}{l}\text { Chemicals used for } \\
\text { boiler water } \\
\text { treatment }\end{array}$ & Trivial & $\begin{array}{l}\text { Boiler water treatment operations, not including cooling } \\
\text { towers. }\end{array}$ \\
\hline $\begin{array}{l}\text { Chemicals used for } \\
\text { oxygen scavenging }\end{array}$ & Trivial & Oxygen scavenging (deaeration of water). \\
\hline $\begin{array}{l}\text { Chemicals used in } \\
\text { bench-scale } \\
\text { chemical analysis }\end{array}$ & Trivial & $\begin{array}{l}\text { Bench-scale laboratory equipment used for physical or } \\
\text { chemical analysis but not lab fume hoods or vents. } \\
\text { Note: This exemption was applied only to biological } \\
\text { research solutions. Otherwise, this exemption was not } \\
\text { applied. }\end{array}$ \\
\hline
\end{tabular}


The following exemptions from NMED/AQB Operating Permit Program "List of Trivial Activities" "were used in the paint analysis:

- Activities that occur strictly for maintenance of grounds or buildings, including the following: lawn care; pest control; grinding; cutting; welding; painting; woodworking; sweeping; general repairs; janitorial activities; plumbing; re-tarring roofs; installing insulation; steam-cleaning and water-washing activities; and paving of roads, parking lots, and other areas.

- Activities for maintenance and repair of equipment, pollution control equipment, or motor vehicles either inside or outside of a building.

- Paint or nonpaint materials dispensed from prepackaged aerosol cans of 16 oz. or less capacity.

The corresponding amounts of paint were totaled for painting activities that did not qualify for one of the trivial activity exemptions listed above. The paint total for 2004 was determined to be $3,044 \mathrm{lb}$ (1.52 tons), which further qualified for the following insignificant activity:

Surface coating of equipment, including spray painting and roll coating, for sources with facility-wide total cleanup solvent and coating actual emissions of less than two (2) tons per year.

All emissions from paints and painting activities were exempt as insignificant or trivial activities and therefore were not included in the 2004 emissions inventory.

\subsection{EMISSIONS SUMMARY}

\subsection{EMISSIONS SUMMARY}

Table 4-1 presents facility-wide actual emissions of criteria pollutants for 2004 as reported in the annual emissions inventory and the semi-annual emissions reports. The Title V Operating Permit emissions limits are also included. Table 4-2 presents actual emissions for HAPs from chemical use. Emission unit information and detailed emissions calculations are included in Attachment A. The 2004 Emissions Inventory Report as submitted to NMED is presented in Attachment B. As mentioned, it is formatted to be compatible with AIRS. Attachment C includes semi-annual emissions reports for 2004.

There are two notable differences in reported emissions in 2004.

- The air curtain destructors, which were a large source of emissions of VOC, PM, CO, and HAPs in 2002 and 2003 were shut down resulting in much lower facility-wide emissions.

Operations for the entire Laboratory were shut down in July 2004 to assess safety and security measures. This shutdown resulted in decreased activities throughout the Laboratory and as a result emissions were also decreased. 
Table 4-1. LANL Facility-Wide Criteria Pollutant Emissions for 2004

\begin{tabular}{|c|c|c|c|}
\hline Pollutant & $\begin{array}{c}\text { Actual Emissions for } \\
\text { Annual Emissions } \\
\text { Reporting (tons/yr) }\end{array}$ & $\begin{array}{c}\text { Actual Emissions for } \\
\text { Semi-Annual Title V } \\
\text { Operating Permit } \\
\text { Reporting (tons/yr) }\end{array}$ & $\begin{array}{c}\text { Title V Operating } \\
\text { Permit Facility-Wide } \\
\text { Emission Limits } \\
\text { (tons/yr) }\end{array}$ \\
\hline $\mathrm{NO}_{\mathrm{x}}$ & 24.5 & 50.5 & 245 \\
\hline $\mathrm{SO}_{\mathrm{x}}$ & 0.34 & 1.5 & 150 \\
\hline $\mathrm{CO}$ & 17.1 & 35.4 & 225 \\
\hline $\mathrm{PM}$ & 3.0 & 4.8 & 120 \\
\hline $\mathrm{PM}_{10}$ & 3.0 & 4.8 & 120 \\
\hline $\mathrm{PM}_{2.5}$ & 2.95 & 4.75 & -- \\
\hline $\mathrm{VOC}$ & 10.0 & 11.4 & 200 \\
\hline
\end{tabular}

Table 4-2. LANL HAP Emissions from Chemical Use for 2004

\begin{tabular}{|l|c|}
\hline \multicolumn{1}{|c|}{ Pollutant } & $\begin{array}{c}\text { Chemical Use HAP } \\
\text { Emissions } \\
\text { (a) } \\
\text { (tons/yr) }\end{array}$ \\
\hline Total HAPs & 5.7 \\
\hline Top 5 HAPs & 1.52 \\
\hline Hydrochloric Acid & 0.73 \\
\hline Methanol & 0.70 \\
\hline Acetonitrile & 0.63 \\
\hline Methylene Chloride & 0.52 \\
\hline Manganese & $\begin{array}{c}\text { HAP emissions from combustion sources are included in the emissions reports, however they are } \\
\text { negligible and do not contribute significantly to facility-wide HAP emissions. }\end{array}$ \\
\hline
\end{tabular}

Figure 4-1 shows criteria air pollutant emissions by source for 2004, excluding the very small emissions sources such as the paper shredder, degreasers, and carpenter shop. As the figure shows, the TA-3 steam plant and the sum of emissions from all small boilers and heaters were the largest sources of $\mathrm{CO}$ and $\mathrm{NO}_{\mathrm{x}}$ emissions in 2004. R\&D chemical use was the largest source of VOC emissions. 


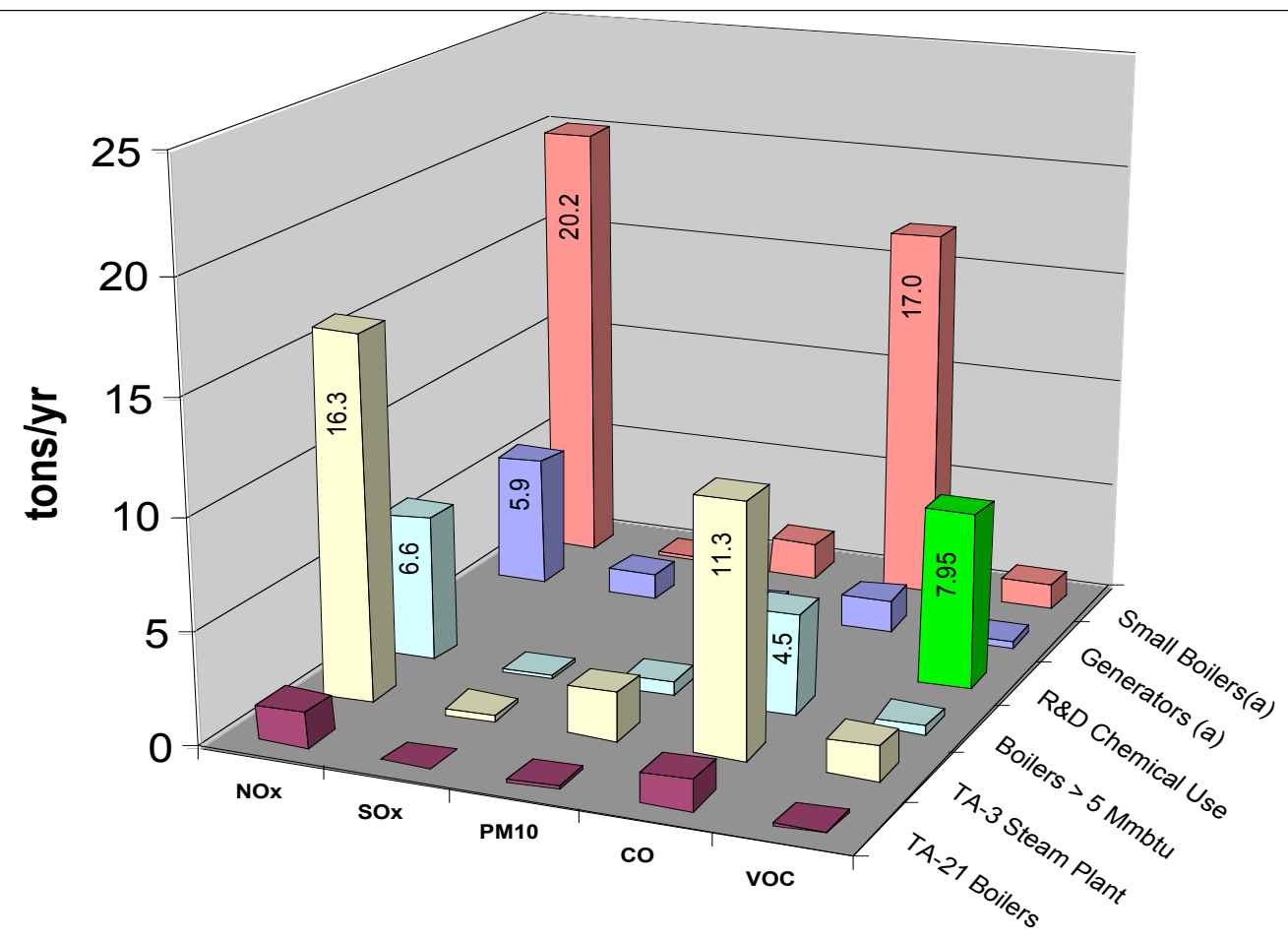

(a) The Generators and Small Boilers are only included in the Title V Operating Emission Report.

Figure 4-1. Emissions of criteria pollutants by source in 2004.

\subsection{Emission Trends And Title V Permit Limits}

A comparison of historical emissions to the facility-wide emission limits in the Title V Operating Permit is provided in the section below. It should be noted that the facility-wide emission limits in the Operating Permit include emissions from some sources that are not included in the annual emissions inventory, most notably small (insignificant) boilers and emergency standby generators. However, historical data are only available for emission sources that were included in the annual emissions inventory submittals.

Figure 4-2 provides a comparison of the past six years' facility-wide emissions for criteria air pollutants as reported to NMED on the annual emission inventory submittal. The facility-wide emission limits included in LANL's Title V Operating Permit are also shown on the graph. 


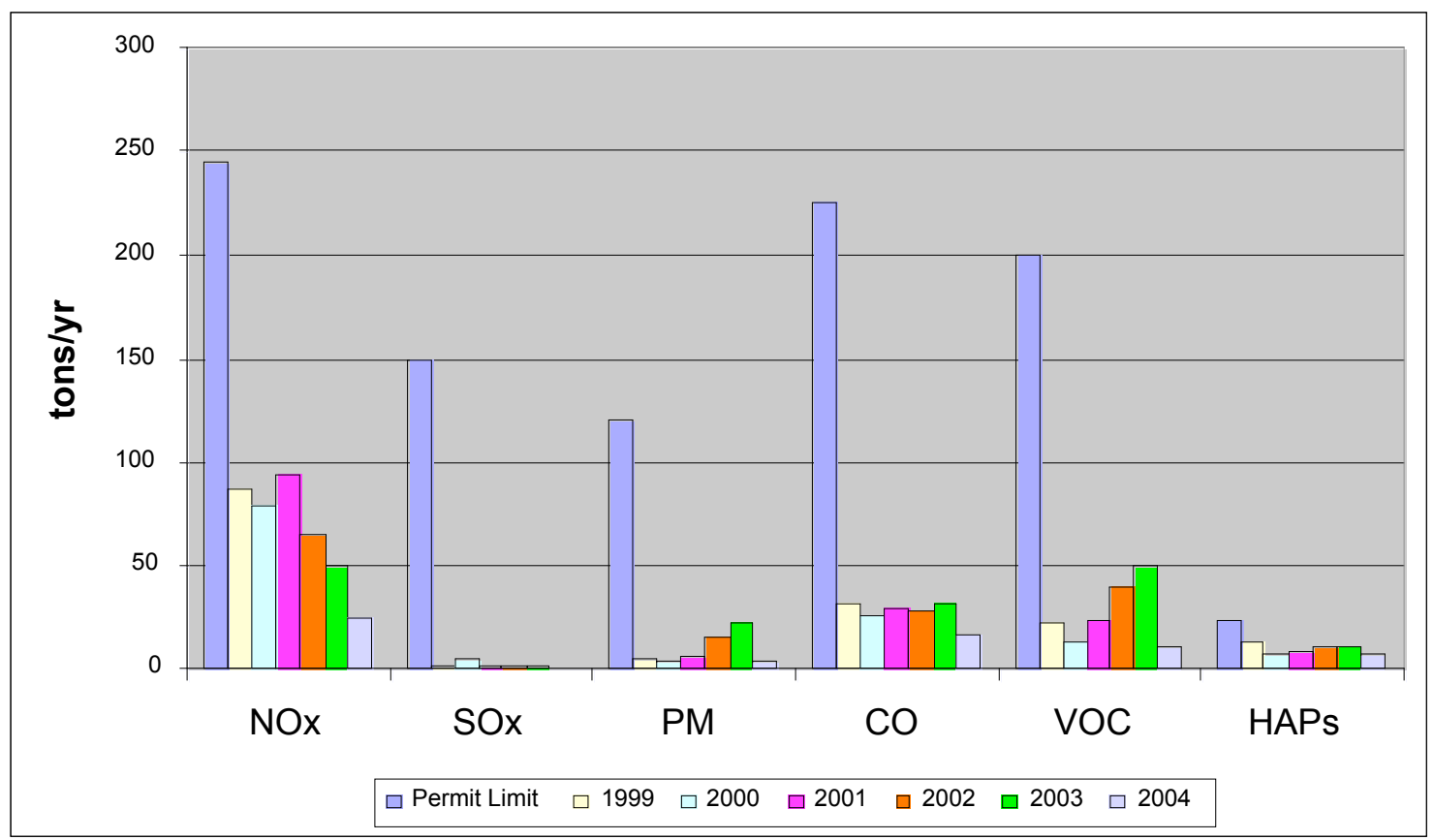

Figure 4-2. Comparison of facility-wide annual reported emissions from 1999-2004.

Figure 4-3 presents VOC and HAP emissions from chemical use activities. As shown, VOC emissions have declined steadily in recent years while HAP emissions from chemical use have remained relatively constant. The continued fluctuation in both VOC and HAP emissions is due to both variations in actual chemical purchases and improvements the Laboratory has made to the chemical tracking system. Emissions in 2004 are lower than previous years due to activities at the Laboratory shutting down in July 2004 as LANL conducted facility-wide security and safety assessments.

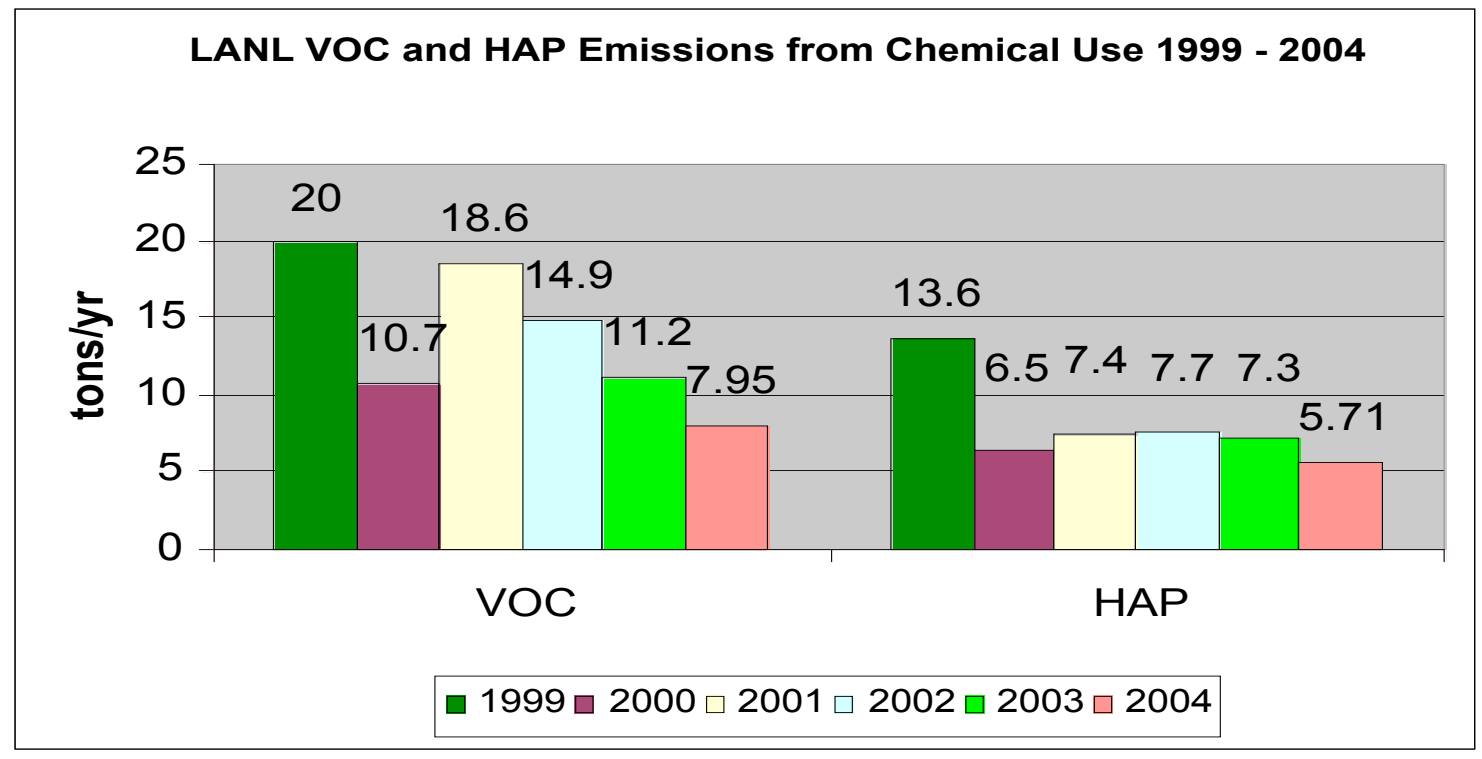

Figure 4-3. VOC and HAP emissions from chemical use, 1999-2004. 


\section{REFERENCES}

1. U.S. Environmental Protection Agency, Aerometric Information Retrieval System (AIRS), http://www.epa.gov/ttn/chief.

2. Los Alamos National Laboratory, Clean Air Act, Title V Operating Permit No. P-100, issued by the New Mexico Environment Department Air Quality Bureau to the University of California, operator of Los Alamos National Laboratory, April 30, 2004.

3. Los Alamos National Laboratory, “2004 Annual Emissions Inventory Report Submittal to the New Mexico Environment Department," LA-UR-05-2050, March 2005.

4. Los Alamos National Laboratory, "Semi-Annual Emissions Report, January-June 2004," submitted to the New Mexico Environment Department, September 2004, LA-UR-046622, and "Semi-Annual Emissions Report, July-December 2004," submitted to the New Mexico Environment Department, March 2005, LA-UR-05-2051.

5. U.S. Environmental Protection Agency, "Compilation of Air Pollutant Emission Factors," AP-42, Fifth Edition, Section 1.4-Natural Gas Combustion, July 1998, and Section 1.3-Fuel Oil Combustion, September 1998.

6. Los Alamos National Laboratory, "20.2.70 NMAC Operating Permit Application for Los Alamos National Laboratory," Chapters 1 \& 2, LA-UR-02-6717; Chapter 3, LA-UR-026258; Chapter 4, LA-UR-02-6601; Maps, LA-UR-02-7202; Application Forms, LA-UR02-7286; November 2002.

7. U.S. Environmental Protection Agency, "Compilation of Air Pollutant Emission Factors," AP-42, Fifth Edition, Section 10.4-Woodworking Waste Collection Operations, February 1980.

8. U.S. Environmental Protection Agency, TANKS Emission Estimation Software, http://www.epa.gov/ttn/chief/software/TANKS.

9. U.S. Environmental Protection Agency, "Compilation of Air Pollutant Emission Factors," AP-42, Fifth Edition, Section 3.3-Gasoline and Diesel Industrial Engines, October 1996, and Section 3.4-Large Stationary Diesel and All Stationary Dual Fired Engines, October 1996.

10. New Mexico Environment Department, Air Quality Bureau, "List of Insignificant Activities under Title V Operating Permits," September 29, 1995.

11. New Mexico Environment Department, Air Quality Bureau, "List of Trivial Activities under Title V Operating Permits," January 10, 1996. 
ATTACHMENT A.

EMISSION CALCULATION WORKSHEETS

FOR INDIVIDUAL EMISSION UNITS 
TA-3 Power Plant Fuel Use Totals 2004 (Data Entry)

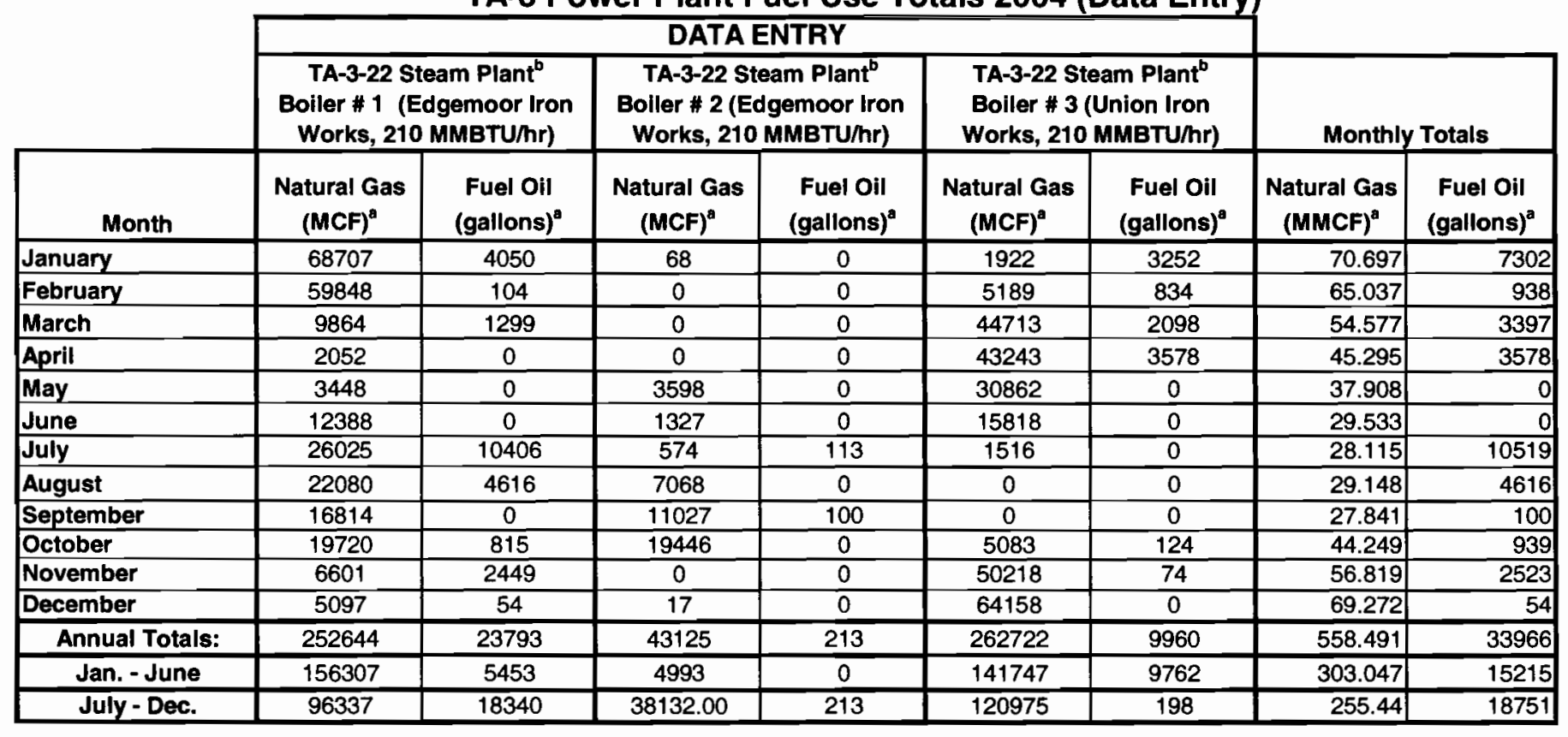

\begin{tabular}{|l|c|c|}
\cline { 2 - 3 } \multicolumn{1}{c|}{} & $\begin{array}{c}\text { 12-Mo. Rolling Total } \\
\text { Natural Gas (MMscf) }\end{array}$ & $\begin{array}{c}\text { 12-Mo. Rolling Total } \\
\text { Fuel Oil (gallons) }\end{array}$ \\
\hline January & 587.6 & 29323 \\
\hline February & 592.4 & 30261 \\
\hline March & 579.6 & 31548 \\
\hline April & 578.1 & 35126 \\
\hline May & 574.3 & 35126 \\
\hline June & 564.6 & 33726 \\
\hline July & 566.3 & 44245 \\
\hline August & 571.0 & 48861 \\
\hline September & 565.3 & 38761 \\
\hline October & 565.1 & 39300 \\
\hline November & 561.1 & 35413 \\
\hline December & 558.5 & 33966 \\
\hline
\end{tabular}

\begin{tabular}{|c|c|c|}
\cline { 2 - 3 } \multicolumn{1}{c|}{} & \multicolumn{2}{c|}{ Totals by Fuel Type } \\
\cline { 2 - 3 } \multicolumn{1}{c|}{} & $\begin{array}{c}\text { Natural Gas } \\
\text { (MMscf) }\end{array}$ & $\begin{array}{c}\text { Fuel Oil } \\
\text { (Gallons) }\end{array}$ \\
\hline Annual Totals: & 558.49 & 33966.00 \\
\hline Jan. - June & 303.05 & 15215.00 \\
\hline July - Dec. & 255.44 & 18751.00 \\
\hline
\end{tabular}

\begin{tabular}{|c|c|c|}
\hline Permit Limits: & 2000 MMscf & 500,000 gallons \\
\hline The limit for Natural Gas is from NSR Permit \# 2195BM2. & The limit for Fuel Oil is from the Title-V Operating Permit.
\end{tabular} 
Emissions by Boiler 2004

\begin{tabular}{|c|c|c|c|c|c|c|c|c|c|c|c|}
\hline \multirow[b]{3}{*}{$\begin{array}{c}\text { Pollutant } \\
\text { Criteria }\end{array}$} & \multicolumn{2}{|c|}{ Emission Factor } & \multirow{2}{*}{\multicolumn{3}{|c|}{$\frac{\text { Unit Emissions }}{\text { Boiler \#1, Stack } 032}$}} & \multirow{2}{*}{\multicolumn{3}{|c|}{$\begin{array}{c}\text { Unit Emissions } \\
\text { Boiler \#2, Stack } 033\end{array}$}} & \multirow{2}{*}{\multicolumn{3}{|c|}{$\begin{array}{c}\text { Unit Emissions } \\
\text { Boiler \#3, Stack } 034\end{array}$}} \\
\hline & \multirow[b]{2}{*}{$\begin{array}{c}\text { Natural } \\
\text { Gas } \\
{\text { (lb/MMscf })^{(a)}}^{(\mathrm{b})}\end{array}$} & \multirow[b]{2}{*}{$\begin{array}{c}\text { Fuel Oil } \\
\text { Pounds/ } \\
1000 \text { gal }\end{array}$} & & & & & & & & & \\
\hline & & & $\begin{array}{l}\text { Annual } \\
\text { (tons) }\end{array}$ & $\begin{array}{c}\text { Jan-June } \\
\text { (tons) }\end{array}$ & $\begin{array}{c}\text { July-Dec } \\
\text { (tons) }\end{array}$ & $\begin{array}{c}\text { Annual } \\
\text { (tons) }\end{array}$ & $\begin{array}{c}\text { Jan-June } \\
\text { (tons) }\end{array}$ & $\begin{array}{c}\text { July-Dec } \\
\text { (tons) }\end{array}$ & $\begin{array}{c}\text { Annual } \\
\text { (tons) }\end{array}$ & $\begin{array}{c}\text { Jan-June } \\
\text { (tons) }\end{array}$ & $\begin{array}{c}\text { July-Dec } \\
\text { (tons) }\end{array}$ \\
\hline Nox ${ }^{(0)}$ & 58 & 8.64 & 7.429 & 4.556 & 2.873 & 1.252 & 0.145 & 1.107 & 7.662 & 4.153 & 3.509 \\
\hline Sox & 0.6 & 7.4 & 0.164 & 0.067 & 0.097 & 0.014 & 0.001 & 0.012 & 0.116 & 0.079 & 0.037 \\
\hline$P M^{(d)}$ & 7.6 & 3.3 & 0.999 & 0.603 & 0.396 & 0.164 & 0.019 & 0.145 & 1.015 & 0.555 & 0.460 \\
\hline PM-10 ${ }^{(d)}$ & 7.6 & 2.3 & 0.987 & 0.600 & 0.387 & 0.164 & 0.019 & 0.145 & 1.010 & 0.550 & 0.460 \\
\hline$P M-2.5^{(d)}$ & 7.6 & 1.55 & 0.978 & 0.598 & 0.380 & 0.164 & 0.019 & 0.145 & 1.006 & 0.546 & 0.460 \\
\hline $\mathrm{CO}^{(e)}$ & 40 & 5.0 & 5.112 & 3.140 & 1.973 & 0.863 & 0.100 & 0.763 & 5.279 & 2.859 & 2.420 \\
\hline VOC & 5.5 & 0.2 & 0.697 & 0.430 & 0.267 & 0.119 & 0.014 & 0.105 & 0.723 & 0.391 & 0.333 \\
\hline HAPS $^{(1)}$ & & & & & & & & & & & \\
\hline Arsenic & 0.0002 & 0.00055 & $3.18 \mathrm{E}-05$ & 1.71E-05 & 1.47E-05 & 4.37E-06 & $4.99 \mathrm{E}-07$ & \begin{tabular}{|l|}
$3.87 \mathrm{E}-06$ \\
\end{tabular} & $2.90 \mathrm{E}-05$ & $1.68 \mathrm{E}-05$ & $1.22 \mathrm{E}-05$ \\
\hline Benzene & 0.0021 & - & $2.65 \mathrm{E}-04$ & $1.64 \mathrm{E}-04$ & $1.01 \mathrm{E}-04$ & $4.53 \mathrm{E}-05$ & $5.24 \mathrm{E}-06$ & $4.00 \mathrm{E}-05$ & $2.76 \mathrm{E}-04$ & $1.49 \mathrm{E}-04$ & $1.27 \mathrm{E}-04$ \\
\hline Beryllium & 0.000012 & 0.00041 & $6.41 E-06$ & $2.06 \mathrm{E}-06$ & $4.35 \mathrm{E}-06$ & $3.03 \mathrm{E}-07$ & $3.00 \mathrm{E}-08$ & $2.73 \mathrm{E}-07$ & $3.62 \mathrm{E}-06$ & $2.86 \mathrm{E}-06$ & $7.67 \mathrm{E}-07$ \\
\hline Cadmium & 0.0011 & 0.00041 & $1.44 \mathrm{E}-04$ & $8.71 E-05$ & $5.68 \mathrm{E}-05$ & $2.38 \mathrm{E}-05$ & $2.75 \mathrm{E}-06$ & $2.10 \mathrm{E}-05$ & $1.47 \mathrm{E}-04$ & $8.00 \mathrm{E}-05$ & $6.66 \mathrm{E}-05$ \\
\hline Chromium & 0.0014 & 0.00041 & $1.82 \mathrm{E}-04$ & $1.11 \mathrm{E}-04$ & $7.12 \mathrm{E}-05$ & $3.02 \mathrm{E}-05$ & $3.50 \mathrm{E}-06$ & $2.67 \mathrm{E}-05$ & $1.86 \mathrm{E}-04$ & $1.01 E-04$ & $8.47 \mathrm{E}-05$ \\
\hline Cobalt & 0.000084 & - & $1.06 \mathrm{E}-05$ & $6.56 \mathrm{E}-06$ & 4.05E-06 & 1.81E-06 & $2.10 \mathrm{E}-07$ & 1.60E-06 & $1.10 \mathrm{E}-05$ & 5.95E-06 & $5.08 \mathrm{E}-06$ \\
\hline Dichlorobenzene & 0.0012 & - & $1.52 \mathrm{E}-04$ & $9.38 \mathrm{E}-05$ & $5.78 \mathrm{E}-05$ & $2.59 \mathrm{E}-05$ & $3.00 \mathrm{E}-06$ & $2.29 \mathrm{E}-05$ & $1.58 \mathrm{E}-04$ & $8.50 \mathrm{E}-05$ & $7.26 \mathrm{E}-05$ \\
\hline Formaldehyde & 0.075 & 0.048 & 1.00E-02 & $5.99 \mathrm{E}-03$ & $4.05 \mathrm{E}-03$ & $1.62 \mathrm{E}-03$ & $1.87 \mathrm{E}-04$ & 1.44E-03 & $1.01 \mathrm{E}-02$ & $5.55 \mathrm{E}-03$ & $4.54 \mathrm{E}-03$ \\
\hline Hexane & 1.8 & - & $2.27 \mathrm{E}-01$ & $1.41 \mathrm{E}-01$ & $8.67 \mathrm{E}-02$ & $3.88 \mathrm{E}-02$ & $4.49 \mathrm{E}-03$ & $3.43 \mathrm{E}-02$ & $2.36 \mathrm{E}-01$ & $1.28 \mathrm{E}-01$ & $1.09 E-01$ \\
\hline Lead & 0.0005 & 0.00123 & $7.78 \mathrm{E}-05$ & $4.24 \mathrm{E}-05$ & $3.54 \mathrm{E}-05$ & $1.09 \mathrm{E}-05$ & $1.25 \mathrm{E}-06$ & $9.66 \mathrm{E}-06$ & $7.18 \mathrm{E}-05$ & $4.15 \mathrm{E}-05$ & $3.04 \mathrm{E}-05$ \\
\hline Mangenese & 0.00038 & 0.00082 & $5.78 \mathrm{E}-05$ & 3.19E-05 & $2.58 \mathrm{E}-05$ & $8.28 \mathrm{E}-06$ & $9.49 \mathrm{E}-07$ & $7.33 \mathrm{E}-06$ & $5.40 \mathrm{E}-05$ & $3.09 \mathrm{E}-05$ & $2.31 \mathrm{E}-05$ \\
\hline Mercury" & 0.00026 & 0.00041 & 3.77E-05 & $2.14 \mathrm{E}-05$ & 1.63E-05 & 5.65E-06 & 6.49E-07 & 5.00E-06 & $3.62 \mathrm{E}-05$ & $2.04 E-05$ & $1.58 \mathrm{E}-05$ \\
\hline Napthalene & 0.00061 & $\cdot$ & 7.71E-05 & 4.77E-05 & $2.94 \mathrm{E}-05$ & $1.32 \mathrm{E}-05$ & $1.52 \mathrm{E}-06$ & $1.16 \mathrm{E}-05$ & $8.01 \mathrm{E}-05$ & $4.32 \mathrm{E}-05$ & $3.69 \mathrm{E}-05$ \\
\hline Nickel & 0.0021 & 0.00041 & $2.70 \mathrm{E}-04$ & $1.65 \mathrm{E}-04$ & $1.05 \mathrm{E}-04$ & $4.53 \mathrm{E}-05$ & $5.24 \mathrm{E}-06$ & 4.01E-05 & $2.78 \mathrm{E}-04$ & $1.51 \mathrm{E}-04$ & $1.27 \mathrm{E}-04$ \\
\hline POM & 0.000088 & 0.0033 & $5.04 \mathrm{E}-05$ & $1.59 \mathrm{E}-05$ & $3.45 \mathrm{E}-05$ & $2.25 \mathrm{E}-06$ & \begin{tabular}{|l|}
$2.20 \mathrm{E}-07$ \\
\end{tabular} & $2.03 \mathrm{E}-06$ & $2.80 \mathrm{E}-05$ & $2.23 \mathrm{E}-05$ & $5.65 \mathrm{E}-06$ \\
\hline Selenium & 0.000024 & 0.00206 & $2.75 \mathrm{E}-05$ & $7.48 \mathrm{E}-06$ & $2.00 \mathrm{E}-05$ & 7.36E-07 & 5.99E-08 & 6.76E-07 & 1.34E-05 & 1.17E-05 & 1.66E-06 \\
\hline Toluene & 0.0034 & $\cdot$ & $4.29 \mathrm{E}-04$ & $2.66 \mathrm{E}-04$ & $1.64 \mathrm{E}-04$ & 7.33E-05 & 8.49E-06 & 6.48E-05 & $4.47 \mathrm{E}-04$ & $2.41 \mathrm{E}-04$ & 2.06E-04 \\
\hline TOTAL HAPS & & & $2.39 \mathrm{E}-01$ & $1.48 \mathrm{E}-01$ & $9.15 \mathrm{E}-02$ & $4.07 \mathrm{E}-02$ & $4.71 \mathrm{E}-03$ & $3.60 \mathrm{E}-02$ & $2.48 \mathrm{E}-01$ & 1.34E-01 & $1.14 \mathrm{E}-01$ \\
\hline
\end{tabular}

For References, see Emission Summary.

Data Reviewed By: 
* 12 Month Rolling Emissions 2004 (Tons)

\begin{tabular}{|l|c|c|r|r|r|r|}
\hline \multicolumn{1}{|c|}{ Pollutant } & \multicolumn{1}{c|}{ TSP } & \multicolumn{1}{c|}{ PM10 } & \multicolumn{1}{c|}{ NOx } & \multicolumn{1}{c|}{ CO } & \multicolumn{1}{c|}{ VOC } & $\mathbf{S O}_{2}$ \\
\hline $\begin{array}{l}\text { Permit Limit (tons/yr) } \\
\text { 12-Month Rolling } \\
\text { Average }\end{array}$ & 15.7 & 15.7 & 99.6 & 81.3 & 11.1 & 36.9 \\
\hline January & & & & & & \\
\hline February & 2.281 & 2.267 & 17.168 & 11.826 & 1.619 & 0.285 \\
\hline March & 2.301 & 2.286 & 17.311 & 11.924 & 1.632 & 0.290 \\
\hline April & 2.255 & 2.239 & 16.945 & 11.671 & 1.597 & 0.290 \\
\hline May & 2.255 & 2.237 & 16.916 & 11.649 & 1.593 & 0.303 \\
\hline June & 2.240 & 2.223 & 16.806 & 11.574 & 1.583 & 0.302 \\
\hline July & 2.201 & 2.184 & 16.519 & 11.376 & 1.556 & 0.294 \\
\hline August & 2.225 & 2.203 & 16.613 & 11.436 & 1.562 & 0.333 \\
\hline September & 2.250 & 2.226 & 16.769 & 11.541 & 1.575 & 0.352 \\
\hline October & 2.212 & 2.193 & 16.560 & 11.402 & 1.558 & 0.313 \\
\hline November & 2.212 & 2.193 & 16.559 & 11.401 & 1.558 & 0.315 \\
\hline December & 2.191 & 2.173 & 16.425 & 11.311 & 1.547 & 0.299 \\
\hline
\end{tabular}

Monthly Emission Totals 2004 (Tons)

\begin{tabular}{|c|c|c|c|c|c|c|}
\hline Pollutant & TSP & PM10 & NOx & Co & VOC & $\mathrm{SO}_{2}$ \\
\hline January & 0.281 & 0.277 & 2.082 & 1.432 & 0.195 & 0.048 \\
\hline February & 0.249 & 0.248 & 1.890 & 1.303 & 0.179 & 0.023 \\
\hline March & 0.213 & 0.211 & 1.597 & 1.100 & 0.150 & 0.029 \\
\hline April & 0.178 & 0.176 & 1.329 & 0.915 & 0.125 & 0.027 \\
\hline May & 0.144 & 0.144 & 1.099 & 0.758 & 0.104 & 0.011 \\
\hline June & 0.112 & 0.112 & 0.856 & 0.591 & 0.081 & 0.009 \\
\hline July & 0.124 & 0.119 & 0.861 & 0.589 & 0.078 & 0.047 \\
\hline August & 0.118 & 0.116 & 0.865 & 0.595 & 0.081 & 0.026 \\
\hline September & 0.106 & 0.106 & 0.808 & 0.557 & 0.077 & 0.009 \\
\hline October & 0.170 & 0.169 & 1.287 & 0.887 & 0.122 & 0.017 \\
\hline November & 0.220 & 0.219 & 1.659 & 1.143 & 0.157 & 0.026 \\
\hline December & 0.263 & 0.263 & 2.009 & 1.386 & 0.191 & 0.021 \\
\hline Annual Totals & 2.178 & 2.161 & 16.343 & 11.255 & 1.539 & 0.293 \\
\hline
\end{tabular}

Data Reviewed By: 
Emission Summary TA-3 Power Plant 2004

\begin{tabular}{|c|c|c|c|c|c|c|c|c|}
\hline \multirow[b]{2}{*}{$\begin{array}{l}\text { Pollutant } \\
\text { Criteria }\end{array}$} & \multicolumn{2}{|c|}{ Emission Factor } & \multirow[b]{2}{*}{$\begin{array}{c}\text { Annual } \\
\text { Emissions } \\
\text { (Natural Gas } \\
+ \text { Fuel Oil) } \\
\text { (tons) }\end{array}$} & \multirow[b]{2}{*}{$\begin{array}{c}\text { Jan-June } \\
\text { Emissions } \\
\text { (Natural } \\
\text { Gas + Fuel } \\
\text { Oil) } \\
\text { (tons) }\end{array}$} & \multirow[b]{2}{*}{$\begin{array}{c}\text { July-Dec } \\
\text { Emissions } \\
\text { (Natural } \\
\text { Gas + Fuel } \\
\text { Oil) } \\
\text { (tons) }\end{array}$} & \multicolumn{2}{|c|}{ Reference } & \multirow[b]{2}{*}{$\begin{array}{l}\text { Reference } \\
\text { (a) AP-42, 7/98, Section. 1.4, Natural Gas Combustion, } \\
\text { Tables 1,4-1, 1.4-2 } \\
\\
\text { (b) Fuel usage obtained from Jerry Gonzales } \\
\text { (FWO-UL). Values are provided in a monthy data } \\
\text { deliverable from KSL. }\end{array}$} \\
\hline & $\begin{array}{c}\text { Natural } \\
\text { Gas } \\
(\mathrm{lb} / \mathrm{MMsc})^{\mathrm{a}}\end{array}$ & $\begin{array}{c}\text { Fuel Oil' } \\
\text { (Ib/1000 } \\
\text { gal.) }\end{array}$ & & & & Gas & Oil & \\
\hline NOx & 58 & 8.64 & 16.343 & 8.854 & 7.489 & (c) & (c) & \multirow{4}{*}{$\begin{array}{l}\text { (c) Average of source tests conducted on all } 3 \text { boilers } \\
\text { September } 2002 \text { buming natural gas after FGR installed. } \\
\text { Assumed FGR resulted in similar Nox reduction for oil. }\end{array}$} \\
\hline sox & 0.6 & 7.39 & 0.293 & $0 . \overline{147}$ & 0.146 & (a)(i) & (g)(i) & \\
\hline $\mathbf{P M}$ & 7.6 & 3.3 & 2.178 & 1.177 & 1.002 & (d) & (d) & \\
\hline PM-10 & 7.6 & 2.3 & 2.161 & 1.169 & 0.992 & (d) & (d) & \\
\hline PM-2.5 & 7.6 & 1.55 & 2.149 & 1.163 & 0.985 & (d) & (d) & \multirow{4}{*}{$\begin{array}{l}\text { (d) All PM from natural gas is assumed <11, so PM-10, } \\
\text { PM-2.5 and total PM have equal EFs, AP-42, Natural } \\
\text { Gas Combustion, Table 1.4-2. The PM emission factor } \\
\text { for fuel oil is the sum of fitterable and condensable PM. }\end{array}$} \\
\hline $\mathrm{CO}$ & 40 & 5.0 & 11.255 & 6.099 & 5.156 & (b) & (g) & \\
\hline VOC & 5.5 & 0.2 & 1.539 & 0.835 & 0.704 & (b) & (i) & \\
\hline \multicolumn{8}{|l|}{ HAPS" } & \\
\hline Arsenic & 0.0002 & 0.00055 & 6.52E-05 & 3.45E-05 & 3.07E-05 & (a) & $(k)$ & \multirow{3}{*}{$\begin{array}{l}\text { (e) AP-42, 1/95, Section. 1.4, Natural Gas Combustion, } \\
\text { Table 1.4-2. Consistent with previous stack tests. }\end{array}$} \\
\hline Benzene & 0.0021 & & $5.86 \mathrm{E}-04$ & $3.18 \mathrm{E}-04$ & $2.68 \mathrm{E}-04$ & (c) & & \\
\hline Beryllium & 0.000012 & 0.00041 & $1.03 \mathrm{E}-05$ & $4.94 \mathrm{E}-06$ & $5.39 \mathrm{E}-06$ & (c) & $(\mathrm{k})$ & \\
\hline Cadmium & 0.0011 & 0.00041 & $3.14 \mathrm{E}-04$ & $1.70 \mathrm{E}-04$ & $1.44 \mathrm{E}-04$ & (c) & (k) & \multirow{3}{*}{$\begin{array}{l}\text { (f) AP-42, 9/98, Section. 1.3, Fuel Oil Combustion, Table } \\
\text { 1.3-1 with Errata, Table 1.3-3, and Table } \\
1.3-6 \text {. }\end{array}$} \\
\hline Chromium & 0.0014 & 0.00041 & $3.98 \mathrm{E}-04$ & $2.15 E-04$ & $1.83 E-04$ & (c) & $(k)$ & \\
\hline Cobalt & 0.000084 & - & 2.35E-05 & $1.27 \mathrm{E}-05$ & 1.07E-05 & (c) & & \\
\hline Dichlorobenzene & 0.0012 & $-\quad$ & 3.35E-04 & $1.82 E-04$ & 1.53E-04 & (c) & & \multirow{5}{*}{$\begin{array}{l}\text { (g) Boilers } 100 \text { MMBtu/hr: } \mathrm{SO} \text { Emission Factor }\left(\mathrm{SO}_{2}\right. \\
\left.(142 \mathrm{~S}\}+\mathrm{SO}_{3}\{5.7 \mathrm{~S})\right)=147.7^{\circ} \mathrm{S} \text { (from AP-42, Table } 1.3 \\
1 \text { w/Errata) }(\mathrm{S}=\text { weight } \% \text { sulfur in oil)(Sulfur content per } \\
\text { analysis on oil in tanks in August 01', no new oil delivered } \\
\text { in 02/03') }\end{array}$} \\
\hline Formaldehyde & 0.075 & 0.048 & $2.18 \mathrm{E}-02$ & $1.17 \mathrm{E}-02$ & $1.00 \mathrm{E}-02$ & (c) & (k) & \\
\hline Hexane & 1.8 & $\cdot$ & $5.03 E-01$ & $2.73 E-01$ & $2.30 \mathrm{E}-01$ & (c) & & \\
\hline Lead & 0.0005 & 0.001233 & $1.61 \mathrm{E}-04$ & $8.51 \mathrm{E}-05$ & $7.54 \mathrm{E}-05$ & (c) & (k) & \\
\hline Mangenese & 0.00038 & 0.000822 & $1.20 \mathrm{E}-04$ & $6.38 \mathrm{E}-05$ & $5.62 E-05$ & (c) & $(k)$ & \\
\hline Mercury & 0.00026 & 0.000411 & $7.96 \mathrm{E}-05$ & $4.25 \mathrm{E}-05$ & $3.71 \mathrm{E}-05$ & (i)(c) & (i)(k) & $S(\%)=0.05$ \\
\hline Napthalene & 0.00061 & - & $1.70 \mathrm{E}-04$ & $9.24 \mathrm{E}-05$ & $7.79 \mathrm{E}-05$ & (c) & & \multirow{4}{*}{$\begin{array}{l}\text { (h) HAP emission factors for natural gas from AP-42, } \\
\text { Tables } 1.4-3 \text { an } 1.4-4 \text {, for fuel oi from AP-42 Tables } 1.3- \\
8 \text { and } 1.3-10 \text {. }\end{array}$} \\
\hline Nickel & 0.0021 & 0.000411 & $5.93 E-04$ & $3.21 \mathrm{E}-04$ & $2.72 \mathrm{E}-04$ & (c) & $(\mathrm{k})$ & \\
\hline POM & 0.000088 & 0.0033 & $8.06 \mathrm{E}-05$ & $3.84 \mathrm{E}-05$ & $4.22 \mathrm{E}-05$ & (c) & $\frac{1}{(k)}$ & \\
\hline Selenium & 0.000024 & 0.002055 & $4.16 \mathrm{E}-05$ & $1.93 \mathrm{E}-05$ & $2.23 \mathrm{E}-05$ & (c) & $(k)$ & \\
\hline Toluene & 0.0034 & 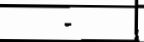 & $9.49 \mathrm{E}-04$ & $5.15 E-04$ & $4.34 \mathrm{E}-04$ & (c) & & \multirow[t]{2}{*}{ (I) AP-42, Table 1.4-2, 1.4-3, and 1.4-4, Juthy 1998} \\
\hline TOTAL HAPS & & & $5.28 E-01$ & $2.87 \mathrm{E}-01$ & 2.42E-01 & & & \\
\hline EPCRA 313 & & & & Ibs./year & & & & \multirow[t]{2}{*}{ (j) Assume all $\mathrm{SO}_{3}$ is converted to sulfuric acid. } \\
\hline Lead & 0.0005 & 0.00123 & $1.61 \mathrm{E}-04$ & 0.321 & & (c) & (i)(k) & \\
\hline Sulfuric Acid & 0.60 & 0.285 & $1.72 \mathrm{E}-01$ & 344.77 & & $(e)(j)$ & $(\mathrm{e})(\mathrm{h})$ & \multirow[t]{2}{*}{ (k) AP-42, tables 1.3-9 and 1.3-10, Seplember 1998.} \\
\hline Mercury & 0.00026 & 0.00041 & $7.96 \mathrm{E}-05$ & 0.159 & & (c) & (i)(k) & \\
\hline PACs & 8.69E-07 & $1.65 \mathrm{E}-05$ & 5.23E-07 & $1.05 E-03$ & & $(f)(I)$ & $(\mathrm{f})(\mathrm{l})$ & (1) EPCRA PAC Guidance Document, Table 2-3. \\
\hline Benzo(g,h,i) perylene & $1.20 \mathrm{E}-06$ & $2.26 \mathrm{E}-06$ & $3.73 \mathrm{E}-07$ & 7.47E-04 & & $(\mathrm{i})(\mathrm{k})(\mathrm{c})$ & (f) & \multirow[t]{2}{*}{ Reviewed By/Date: } \\
\hline Zinc & 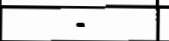 & 0.00055 & 9.31E-06 & $1.86 \mathrm{E}-02$ & & & $(\mathrm{k})$ & \\
\hline
\end{tabular}




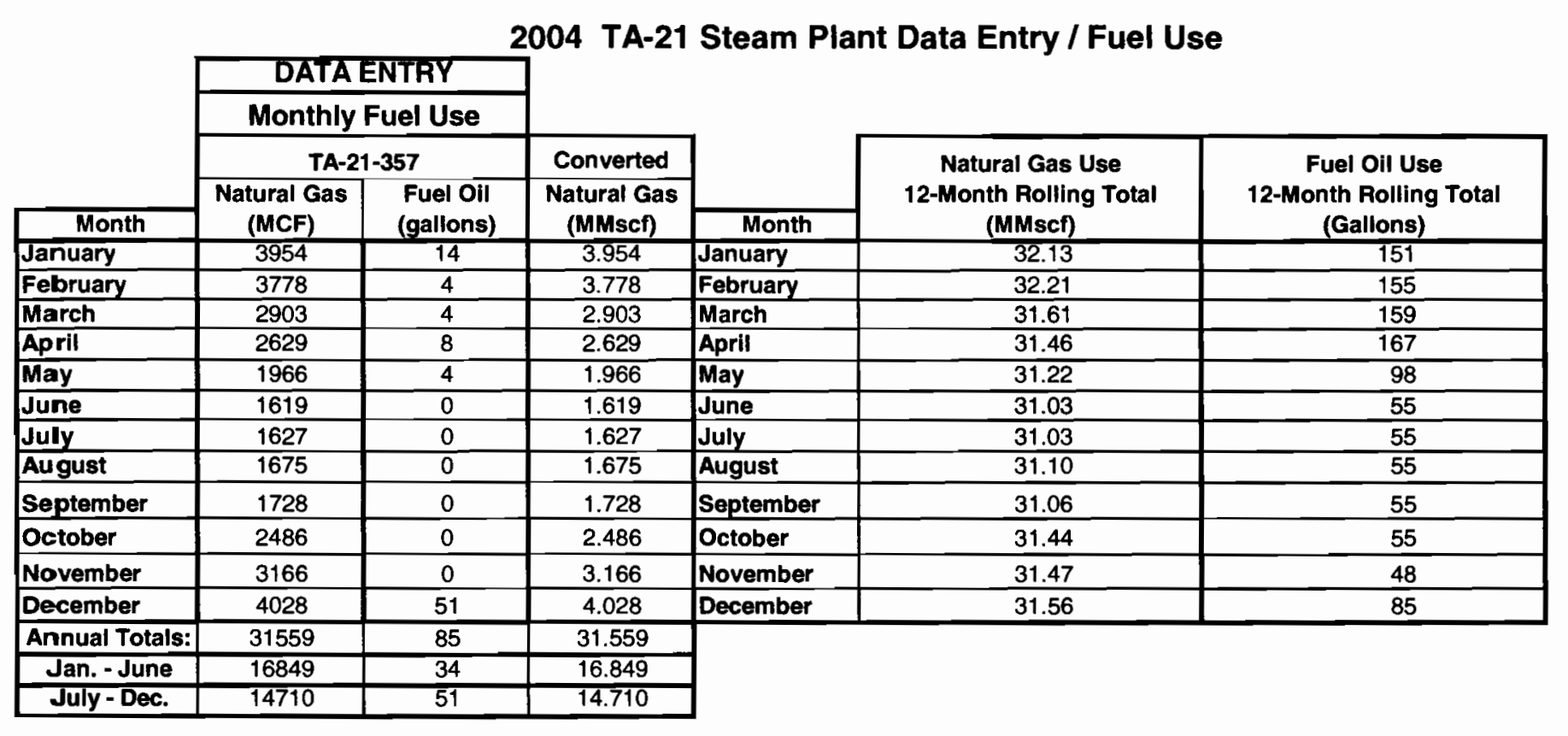

Permit Limit = 60 MMScf/yr natural gas (12 month rolling total) and $10,000 \mathrm{gal} / \mathrm{yr}$ fuel oil (12 month rolling total) 
2004 TA-21 Steam Plant Emission Summary

\begin{tabular}{|c|c|c|c|}
\hline $\begin{array}{l}\text { Pollutant } \\
\text { Criteria }\end{array}$ & $\begin{array}{c}\text { Annual Emissions } \\
\text { (Natural Gas + Fuel Oil) } \\
\text { (tons) }\end{array}$ & $\begin{array}{c}\text { Jan-June Emissions } \\
\text { (Natural Gas + Fuel Oil) } \\
\text { (tons) }\end{array}$ & $\begin{array}{c}\text { July-Dec Emissions } \\
\text { (Natural Gas + Fuel Oil) } \\
\text { (tons) }\end{array}$ \\
\hline NOx & 1.579 & 0.843 & 0.736 \\
\hline sox & 0.012 & 0.006 & 0.006 \\
\hline PM & 0.120 & 0.064 & 0.056 \\
\hline PM-10 & 0.120 & 0.064 & 0.056 \\
\hline PM-2.5 & 0.120 & 0.064 & 0.056 \\
\hline $\mathrm{CO}$ & 1.326 & 0.708 & 0.618 \\
\hline VOC & 0.087 & 0.046 & 0.040 \\
\hline \multicolumn{4}{|l|}{ HAPs } \\
\hline Arsenic & $3.18 E-06$ & $1.69 E-06$ & $1.48 \mathrm{E}-06$ \\
\hline Benzene & $3.31 \mathrm{E}-05$ & $1.77 \mathrm{E}-05$ & $1.54 \mathrm{E}-05$ \\
\hline Beryllium & $2.07 \mathrm{E}-07$ & $1.08 \mathrm{E}-07$ & $9.87 \mathrm{E}-08$ \\
\hline Cadmium & $1.74 \mathrm{E}-05$ & 9.27E-06 & $8.10 \mathrm{E}-06$ \\
\hline Chromium & $2.21 \mathrm{E}-05$ & $1.18 \mathrm{E}-05$ & 1.03E-05 \\
\hline Cobalt & 1.33E-06 & 7.08E-07 & $6.18 \mathrm{E}-07$ \\
\hline Dichlorobenzene & $1.89 \mathrm{E}-05$ & $1.01 \mathrm{E}-05$ & 8.83E-06 \\
\hline Formaldehyde & $1.19 \mathrm{E}-03$ & $6.33 \mathrm{E}-04$ & $5.53 \mathrm{E}-04$ \\
\hline Hexane & $2.84 \mathrm{E}-02$ & $1.52 \mathrm{E}-02$ & $1.32 \mathrm{E}-02$ \\
\hline Lead & 7.94E-06 & 4.23E-06 & 3.71E-06 \\
\hline Mangenese & $6.03 \mathrm{E}-06$ & $3.22 \mathrm{E}-06$ & $2.82 \mathrm{E}-06$ \\
\hline Mercury & $4.12 \mathrm{E}-06$ & $2.20 \mathrm{E}-06$ & $1.92 \mathrm{E}-06$ \\
\hline Napthalene & $9.63 \mathrm{E}-06$ & $5.14 \mathrm{E}-06$ & $4.49 \mathrm{E}-06$ \\
\hline Nicke! & $3.32 E-05$ & $1.77 \mathrm{E}-05$ & $1.55 \mathrm{E}-05$ \\
\hline POM & $1.53 \mathrm{E}-06$ & 7.97E-07 & 7.31E-07 \\
\hline Selenium & $4.66 \mathrm{E}-07$ & $2.37 \mathrm{E}-07$ & $2.29 \mathrm{E}-07$ \\
\hline Toluene & 5.37E-05 & $2.86 \mathrm{E}-05$ & $2.50 \mathrm{E}-05$ \\
\hline Zinc & \#REF! & \#REF! & \#REF! \\
\hline \begin{tabular}{|l} 
TOTAL HAPS \\
\end{tabular} & $2.98 \mathrm{E}-02$ & \#REF! & \#REF! \\
\hline EPCRA 313 & & Ibs./year & \\
\hline Lead & 7.94E-06 & 0.016 & \\
\hline Sulfuric Acid & $9.47 \mathrm{E}-03$ & 18.94 & \\
\hline Mercury & 4.12E-06 & 0.008 & \\
\hline PACs & $1.44 \mathrm{E}-08$ & $2.88 \mathrm{E}-05$ & \\
\hline Benzo $(g, h, i)$ perylene & $1.90 \mathrm{E}-08$ & $3.81 \mathrm{E}-05$ & \\
\hline
\end{tabular}


2004 TA-21 Steam Plant Emissions Calculations

\begin{tabular}{|c|c|c|c|c|c|c|c|c|c|c|c|}
\hline & \multicolumn{5}{|c|}{ Natural Gas } & \multicolumn{5}{|c|}{ Fuel Oil } & \multirow{2}{*}{\begin{tabular}{|l|}
\multicolumn{1}{|c|}{ Reference } \\
(a) Fuel usage obtained from Jery \\
Gonzales, FWO-UI
\end{tabular}} \\
\hline $\begin{array}{c}\text { Pollutant } \\
\text { Criteria }\end{array}$ & $\begin{array}{c}\text { Emission } \\
\text { Factor } \\
\text { (Ib/MMscf) }\end{array}$ & $\begin{array}{c}\text { Annual } \\
\text { Emission } \\
\mathrm{s} \text { (tons) }\end{array}$ & $\begin{array}{c}\text { Emissions } \\
\text { (tons) } \\
\text { Jan-June }\end{array}$ & $\begin{array}{c}\text { Emissions } \\
\text { (tons) } \\
\text { July-Dec }\end{array}$ & Ref. & $\begin{array}{c}\text { Emission } \\
\text { Factor } \\
(\mathrm{lb} / 1000 \mathrm{gal})\end{array}$ & $\begin{array}{c}\text { Annual } \\
\text { Emissions } \\
\text { (tons) } \\
\end{array}$ & \begin{tabular}{|c|} 
Emissions \\
(tons) \\
Jan-June
\end{tabular} & $\begin{array}{c}\text { Emissions } \\
\text { (tons) } \\
\text { July-Dec }\end{array}$ & Ret. & \\
\hline Nox & 100 & 1.578 & 0.842 & 0.736 & (b) & 20 & $8.50 \mathrm{E}-04$ & $3.40 \mathrm{E}-04$ & $5.10 \mathrm{E}-04$ & (g) & \multirow{3}{*}{$\begin{array}{l}\text { (b) AP-42, 7/98, Section. 1.4, } \\
\text { Natural Gas Combustion, Tables } \\
1.4-1,1.4-2 \text {. }\end{array}$} \\
\hline SOx & 0.6 & 0.009 & 0.005 & 0.004 & (b) & 49.0 & $2.08 \mathrm{E}-03$ & $8.32 \mathrm{E}-04$ & $1.25 \mathrm{E}-03$ & (h) & \\
\hline PM & 7.6 & 0.120 & 0.064 & 0.056 & (b) & 3.3 & $1.40 \mathrm{E}-04$ & $5.61 \mathrm{E}-05$ & $8.42 \mathrm{E}-05$ & (g) & \\
\hline$P M-10$ & 7.6 & 0.120 & 0.064 & 0.056 & (d) & 2.3 & $9.78 \mathrm{E}-05$ & $3.91 \mathrm{E}-05$ & 5.87E-05 & (i) & \multirow{3}{*}{$\begin{array}{l}\text { (c) AP-42 7/98, Section 1.4, } \\
\text { Natural Gas Combustion, Tables } \\
1.4-3,1.4-4 \text {. }\end{array}$} \\
\hline$P M-2.5$ & 7.6 & 0.120 & 0.064 & 0.056 & (d) & 1.55 & $6.59 \mathrm{E}-05$ & $2.64 \mathrm{E}-05$ & $3.95 \mathrm{E}-05$ & (j) & \\
\hline CO & 84 & 1.325 & 0.708 & 0.618 & (b) & 5.0 & $2.13 \mathrm{E}-04$ & $8.50 \mathrm{E}-05$ & $1.28 \mathrm{E}-04$ & (g) & \\
\hline VOC & 5.5 & 0.087 & 0.046 & 0.040 & (b) & 0.2 & $8.50 \mathrm{E}-06$ & $3.40 \mathrm{E}-06$ & $5.10 \mathrm{E}-06$ & (i) & \multirow{4}{*}{$\begin{array}{l}\text { (d) PM-10 and PM-2.5 for natural } \\
\text { gas combustion roughny equal to } \\
\text { PM, per AP-42, Natural Gas } \\
\text { Combustion, Table 1.4-2. }\end{array}$} \\
\hline HAPS & & & & & & & & & & & \\
\hline Arsenic & 0.0002 & $3.16 \mathrm{E}-06$ & $1.68 \mathrm{E}-06$ & $1.47 \mathrm{E}-06$ & (c) & 0.00055 & $2.33 \mathrm{E}-08$ & $9.32 \mathrm{E}-09$ & $1.40 \mathrm{E}-08$ & (k) & \\
\hline Benzene & 0.0021 & $3.31 \mathrm{E}-05$ & $1.77 \mathrm{E}-05$ & $1.54 \mathrm{E}-05$ & (c) & & & & & & \\
\hline Beryllium & 0.000012 & 1.89E-07 & $1.01 \mathrm{E}-07$ & 8.83E-08 & (c) & 0.00041 & $1.75 \mathrm{E}-08$ & $6.99 \mathrm{E}-09$ & $1.05 \mathrm{E}-08$ & (k) & \multirow{2}{*}{$\begin{array}{l}\text { (e) Assume all SOx is converted to } \\
\text { sulfuric acid. }\end{array}$} \\
\hline Cadmium & 0.0011 & $1.74 \mathrm{E}-05$ & $9.27 \mathrm{E}-06$ & 8.09E-06 & (c) & 0.00041 & $1.75 \mathrm{E}-08$ & $6.99 \mathrm{E}-09$ & $1.05 \mathrm{E}-08$ & (k) & \\
\hline Chromium & 0.0014 & $2.21 E-05$ & 1.18E-05 & $1.03 E-05$ & (c) & 0.00041 & $1.75 \mathrm{E}-08$ & $6.99 E-09$ & $1.05 E-08$ & (k) & \multirow{2}{*}{$\begin{array}{l}\text { (f) EPCRA PAC Guidance } \\
\text { Document, Table 2-3. }\end{array}$} \\
\hline Cobalt & 0.000084 & 1.33E-06 & $7.08 \mathrm{E}-07$ & $6.18 E-07$ & (c) & & & & & & \\
\hline Dichlorobenzene & 0.0012 & $1.89 \mathrm{E}-05$ & $1.01 \mathrm{E}-05$ & 8.83E-06 & (c) & & & & & & \multirow{4}{*}{$\begin{array}{l}\text { (g) AP-42, 9/98, Section. } 1.3 \text {, Fuel } \\
\text { Oil Combustion, Table } 1.3-1 \text { with } \\
\text { Errata. The PM emission factor is } \\
\text { the sum of fitterable and } \\
\text { condensable PM. }\end{array}$} \\
\hline Formaldehyde & 0.075 & $1.18 \mathrm{E}-03$ & $6.32 \mathrm{E}-04$ & $5.52 \mathrm{E}-04$ & (c) & 0.048 & $2.04 \mathrm{E}-06$ & $8.16 \mathrm{E}-07$ & $1.22 \mathrm{E}-06$ & (k) & \\
\hline Hexane & 1.8 & $2.84 \mathrm{E}-02$ & $1.52 \mathrm{E}-02$ & $1.32 E-02$ & (c) & & & & & & \\
\hline Lead & 0.0005 & $7.89 \mathrm{E}-06$ & 4.21E-06 & $3.68 \mathrm{E}-06$ & (c) & 0.00123 & $5.24 \mathrm{E}-08$ & $2.10 \mathrm{E}-08$ & $3.14 \mathrm{E}-08$ & (k) & \\
\hline Mangenese & 0.00038 & $6.00 \mathrm{E}-06$ & $3.20 \mathrm{E}-06$ & $2.79 \mathrm{E}-06$ & (c) & 0.00082 & $3.49 \mathrm{E}-08$ & $1.40 \mathrm{E}-08$ & $2.10 \mathrm{E}-08$ & (k) & \multirow{4}{*}{$\begin{array}{l}\text { (h) } \mathrm{S}=\text { weight \% sulfur in oil (Title } \\
\text { V Application, December 1995) } \\
\text { Boilers }<100 \text { MMBtwhr: SOx } \\
\text { Emission Factor }=144 * \mathrm{~S}\end{array}$} \\
\hline Mercury & 0.00026 & $4.10 \mathrm{E}-06$ & $2.19 \mathrm{E}-06$ & $1.91 \mathrm{E}-06$ & (c) & 0.00041 & $1.75 \mathrm{E}-08$ & $6.99 \mathrm{E}-09$ & $1.05 \mathrm{E}-08$ & (k) & \\
\hline Napthalene & 0.00061 & $9.63 E-06$ & $5.14 \mathrm{E}-06$ & $4.49 E-06$ & (c) & & & & & & \\
\hline Nickel & 0.0021 & $3.31 \mathrm{E}-05$ & $1.77 \mathrm{E}-05$ & $1.54 \mathrm{E}-05$ & (c) & 0.00041 & $1.75 \mathrm{E}-08$ & $6.99 \mathrm{E}-09$ & $1.05 \mathrm{E}-08$ & (k) & \\
\hline$\overline{\text { POM }}$ & 0.000088 & $1.39 \mathrm{E}-06$ & $7.41 \mathrm{E}-07$ & $6.47 \mathrm{E}-07$ & (c) & 0.0033 & $1.40 \mathrm{E}-07$ & $5.61 \mathrm{E}-08$ & $8.42 \mathrm{E}-08$ & (k) & $S(\%)=0.34$ \\
\hline Selenium & 0.000024 & $3.79 \mathrm{E}-07$ & $2.02 E-07$ & $1.77 \mathrm{E}-07$ & (c) & 0.00206 & $8.73 \mathrm{E}-08$ & $3.49 \mathrm{E}-08$ & $5.24 \mathrm{E}-08$ & (k) & \multirow{3}{*}{$\begin{array}{l}\text { (i) AP-42, 9198, Section 1.3, Fuel } \\
\text { Oil Combustion, Table 1.3-3 } \\
\text { NMTOC. }\end{array}$} \\
\hline \begin{tabular}{|l|} 
Toluene \\
\end{tabular} & 0.0034 & 5.37E-05 & $2.86 \mathrm{E}-05$ & $2.50 \mathrm{E}-05$ & (c) & & & & & & \\
\hline TOTAL HAPS & & $2.98 \mathrm{E}-02$ & $1.59 \mathrm{E}-02$ & $1.39 \mathrm{E}-02$ & & & $2.47 E-06$ & $9.86 \mathrm{E}-07$ & $1.48 \mathrm{E}-06$ & & \\
\hline EPCRA 313 & & & Ibs./year & & & & & Ibs./year & & & \multirow{4}{*}{$\begin{array}{l}\text { (i) AP-42 9/98, Section 1.3, Fuel Oi } \\
\text { Combustion, Table 1.3-6. The PM } \\
\text { emission factor is the sum of } \\
\text { fitterable and condensable PM. }\end{array}$} \\
\hline Lead & 0.0005 & $7.89 \mathrm{E}-06$ & 0.016 & & (c) & 0.00123 & $5.24 \mathrm{E}-08$ & 1.05E-04 & & (k) & \\
\hline Sulfuric Acid & 0.60 & $9.47 \mathrm{E}-03$ & 18.935 & & (e) & 0.0 & $0.00 \mathrm{E}+00$ & 0.000 & & (e) $(\mathrm{h}$ & \\
\hline Mercury & 0.00026 & $4.10 \mathrm{E}-06$ & $8.21 \mathrm{E}-03$ & & (c) & 0.00041 & $1.75 \mathrm{E}-08$ & $3.49 \mathrm{E}-05$ & & (k) & \\
\hline PACs & $8.69 \mathrm{E}-07$ & $1.37 \mathrm{E}-08$ & $2.74 \mathrm{E}-05$ & & $(f)$ & $1.65 \mathrm{E}-05$ & $7.01 \mathrm{E}-10$ & $1.40 \mathrm{E}-06$ & & (f) & \multirow{2}{*}{$\begin{array}{l}\text { (k) AP-42, 9/98, Section } 1.3 \text {, Fuel } \\
\text { Oil Combustion, Tables } 1.3-8-1.3- \\
10 \text {. }\end{array}$} \\
\hline Benzo(g,h,i) perylene & $1.20 \mathrm{E}-06$ & $1.89 \mathrm{E}-08$ & $3.79 \mathrm{E}-05$ & & (c) & $2.26 \mathrm{E}-06$ & $9.61 \mathrm{E}-11$ & $1.92 \mathrm{E}-07$ & & (f) & \\
\hline
\end{tabular}


2004 Small Boilers Data Entry / Gas Use

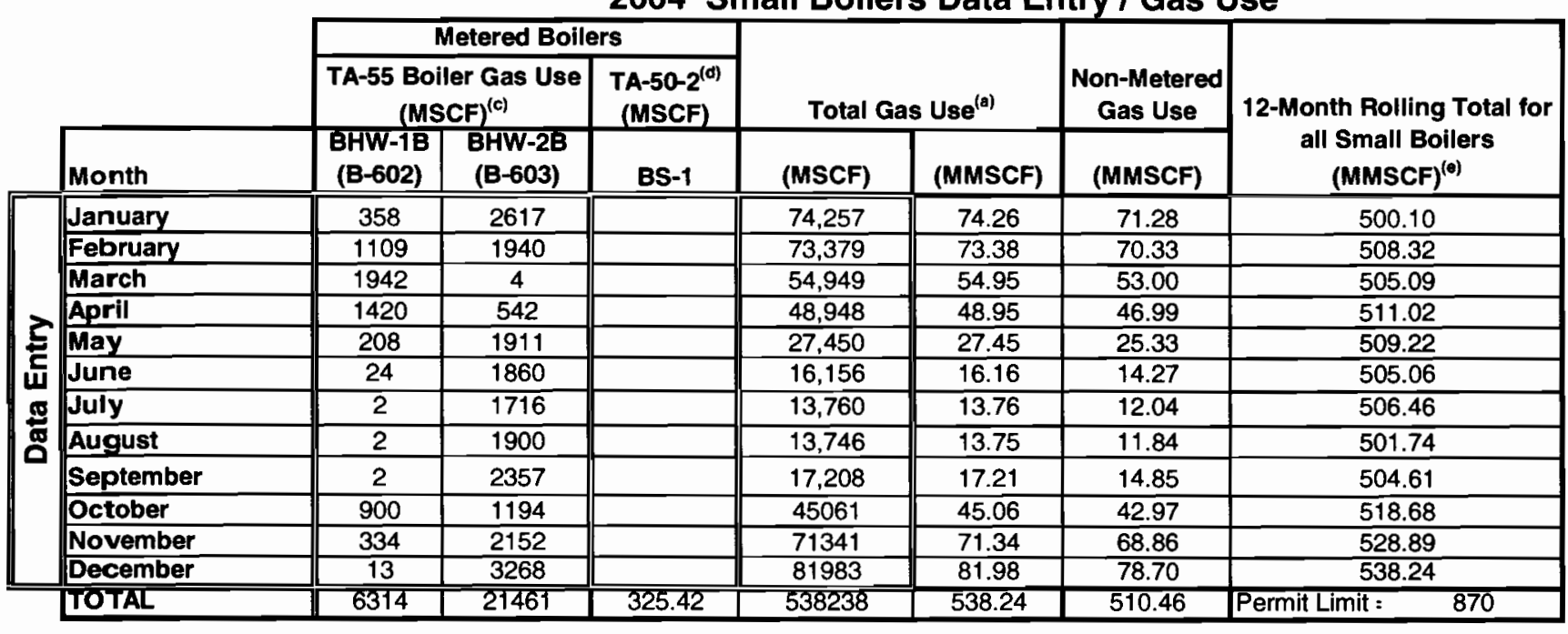

\begin{tabular}{lccc}
\hline 2004 Non Metered Boiler Pool Capacity: & 262.1 & MMBTU/hr $^{(1)}$ & \\
\hline Estimated Gas-Use per MMBtu rating Jan-June: & 1.07 & MMsct/MMBtu/hr \\
Estimated Gas-Use per MMBtu rating July-Dec: & & 0.87 & MMscf/MMBtu/hr \\
Estimated Gas-Use per MMBtu - Annual & 1.95 & MMsct/MMBtu/hr
\end{tabular}

Definitions: $\quad$ MMSCF= Million Standard Cubic Feet

MSCF = Thousand Standard Cubic Feet

Metered/Non-metered: Metered boilers are those units that have unit specific volumetric flow meters for the

boiler(s) only.

\begin{tabular}{|c|c|c|c|c|c|c|c|c|c|}
\hline & \multicolumn{9}{|c|}{ Gas Use Non-Metered ${ }^{(g)}$ (MMSCF) } \\
\hline ARS Stack \# & 015 & 016 & 017 & 018 & 019 & 020 & 021 & 024 & Units $^{(\mathbf{h})}$ \\
\hline Location: & TA-48-1 & TA-48-1 & TA-48-1 & TA-53-365 & TA-53-365 & TA-59-1 & TA-59-1 & TA-16-1484 & Lab Wide \\
\hline 1D: & BS-1 & BS-2 & BS-6 & BHW-1 & BHW-2 & BHW-1 & BHW-2 & Plant 5 & Various \\
\hline Design Rate (I) (MMBTU/hr) & 5.336 & 5.335 & 7.140 & 7.115 & 7.115 & 5.335 & 5.335 & 12.700 & 207 \\
\hline Calculated Gas Use-Jan-June & 5.725 & 5.724 & 7.660 & 7.633 & 7.633 & 5.724 & 5.724 & 13.626 & 221.753 \\
\hline Calculated Gas Use-July-Dec & 4.668 & 4.667 & 6.245 & 6.223 & 6.223 & 4.667 & 4.667 & 11.109 & 180.790 \\
\hline Calculated Gas Use-Annual & 10.393 & 10.391 & 13.906 & 13.856 & 13.856 & 10.391 & 10.391 & 24.734 & 402.544 \\
\hline
\end{tabular}




\begin{tabular}{|c|c|c|c|}
\hline \multicolumn{4}{|c|}{ Emission Factors (lb/MMsct) } \\
\hline Criteria Pollutant & $\begin{array}{c}\text { Small } \\
\text { Uncontrolied } \\
\text { Boilers }^{1}\end{array}$ & $\begin{array}{c}\text { TA-16 Low } \\
\text { NOx } \\
\text { Boilers }\end{array}$ & $\begin{array}{l}\text { TA-55-6 } \\
\text { Boilers }^{3}\end{array}$ \\
\hline NOx & 100 & 37.08 & 138 \\
\hline sox & 0.6 & 0.6 & 0.6 \\
\hline $\mathrm{PM}^{2}$ & 7.6 & 7.6 & 14.2 \\
\hline$P M-10^{2}$ & 7.6 & 7.6 & 14.2 \\
\hline PM-2.5 ${ }^{2}$ & 7.6 & 7.6 & 14.2 \\
\hline co & 84 & 37.08 & 38.2 \\
\hline voc & 5.5 & 5.5 & 5.98 \\
\hline \multicolumn{4}{|l|}{ HAPs $^{5}$} \\
\hline Arsenic & 0.0002 & & \\
\hline Benzene & 0.0021 & & \\
\hline BE & 0.000012 & & \\
\hline Cadmium & 0.0011 & & \\
\hline Chromium & 0.0014 & & \\
\hline Cobalt & 0.000084 & & \\
\hline Dichlorobenzene & 0.0012 & & \\
\hline Formaldehyde & 0.075 & & \\
\hline Hexane & 1.8 & & \\
\hline Lead & 0.0005 & & \\
\hline Mangenese & 0.00038 & & \\
\hline Mercury & 0.00026 & & \\
\hline Napthalene & 0.00061 & & \\
\hline \begin{tabular}{|l|} 
Nickel \\
201
\end{tabular} & 0.0021 & & \\
\hline POM & 0.000088 & & \\
\hline Selenium & 0.000024 & & \\
\hline Toluene & 0.0034 & & \\
\hline
\end{tabular}

References for Emission Factors

(1) AP-42, 7/98, Section 1.4, Natural Gas Combustion, Small Boilers.
(2) Emission factors for natural gas of PM-10 and PM-2.5 are roughy equal to those of PM, Natural Gas Combustion, Table 1.4-2

(3) AP-42, 7/98, Section 1.4 Natural Gas Combustion, Small Boilers for SOx. Stack test on 3/00 for NOx. Otherwise, Emission factors from Sellers Engineering Co.

(3) AP-42, 7/98, Section 1.4, Natural Gas Combustion, Small Boilers for SOx. Stack test on 3/00 for NOx. Otherwise, Emission factors from Sellers Engine

(4) AP-42, 7/98, Section 1.4, Natural Gas Combustion, Small Boilers; Emission factors for NOx and CO from

2004 El-04 Small Boil 


\begin{tabular}{|c|c|c|c|}
\hline \multicolumn{4}{|c|}{$\begin{array}{l}2004 \text { Small Boilers Emission Summary } \\
\text { Title V Semi-Annual Reporting }\end{array}$} \\
\hline & \multicolumn{3}{|c|}{\begin{tabular}{|c|} 
Total Emissions (tons) \\
\end{tabular}} \\
\hline $\begin{array}{l}\text { Pollutant } \\
\text { Criteria }\end{array}$ & $\begin{array}{c}\text { Annual Emissions } \\
\text { (Includes } \\
\text { Insignificant } \\
\text { Sources) }\end{array}$ & $\begin{array}{l}\text { Jan-June } \\
\text { (Includes } \\
\text { Insignificant } \\
\text { Sources) }\end{array}$ & $\begin{array}{l}\text { July-Dec } \\
\text { (Includes } \\
\text { Insignificant } \\
\text { Sources) }\end{array}$ \\
\hline NOX & 26.678 & 14.601 & 12.077 \\
\hline SOx & 0.162 & 0.089 & 0.073 \\
\hline $\mathbf{P M}$ & 2.138 & 1.168 & 0.970 \\
\hline PM-10 & 2.138 & 1.168 & 0.970 \\
\hline PM-2.5 & 2.138 & 1.168 & 0.970 \\
\hline CO & 21.403 & 11.764 & 9.639 \\
\hline VOC & 1.488 & 0.815 & 0.672 \\
\hline \multicolumn{4}{|l|}{ HAPs } \\
\hline Arsenic & $5.39 \mathrm{E}-05$ & $2.95 \mathrm{E}-05$ & $2.43 \mathrm{E}-05$ \\
\hline Benzene & $5.65 \mathrm{E}-04$ & $3.10 \mathrm{E}-04$ & $2.55 \mathrm{E}-04$ \\
\hline BE & $3.23 \mathrm{E}-06$ & 1.77E-06 & $1.46 \mathrm{E}-06$ \\
\hline Cadmium & $2.96 \mathrm{E}-04$ & $1.62 \mathrm{E}-04$ & $1.34 \mathrm{E}-04$ \\
\hline Chromium & 3.77E-04 & 2.07E-04 & $1.70 \mathrm{E}-04$ \\
\hline Cobalt & $2.26 \mathrm{E}-05$ & $1.24 \mathrm{E}-05$ & $1.02 E-05$ \\
\hline Dichlorobenzene & $3.23 E-04$ & 1.77E-04 & 1.46E-04 \\
\hline Formaldehyde & 2.02E-02 & $1.11 \mathrm{E}-02$ & $9.12 E-03$ \\
\hline Hexane & $4.85 \mathrm{E}-01$ & $2.66 \mathrm{E}-01$ & $2.19 \mathrm{E}-01$ \\
\hline Lead & $1.35 \mathrm{E}-04$ & $7.38 \mathrm{E}-05$ & $6.08 \mathrm{E}-05$ \\
\hline Mangenese & $1.02 \mathrm{E}-04$ & 5.61E-05 & $4.62 E-05$ \\
\hline Mercury & $7.00 \mathrm{E}-05$ & $3.84 \mathrm{E}-05$ & $3.16 \mathrm{E}-05$ \\
\hline Napthalene & $1.64 \mathrm{E}-04$ & $9.01 \mathrm{E}-05$ & 7.42E-05 \\
\hline Nickel & $5.65 \mathrm{E}-04$ & $3.10 \mathrm{E}-04$ & $2.55 \mathrm{E}-04$ \\
\hline POM & 2.37E-05 & $1.30 \mathrm{E}-05$ & 1.07E-05 \\
\hline Selenium & $6.46 \mathrm{E}-06$ & $3.54 \mathrm{E}-06$ & 2.92E-06 \\
\hline Toluene & $9.16 \mathrm{E}-04$ & $5.02 E-04$ & 4.14E-04 \\
\hline TOTAL HAPS & 0.509 & 0.279 & 0.230 \\
\hline
\end{tabular}


(b)TA-16 Boilers include 2 boilers in plant 5 . Gas use was difficult to obtain, so, the boilers were included in the "boiler pool" to determine gas use. Plant 6 has been taken off line and is not expected to be reused or boilers relocated. The removal of these boilers will be requested in the next operating permit revision.

(c) TA-55 has two boilers with separate AIRs numbers. Each boiler has a gas meter. The gas use information is provided monthly by the TA-55 facility personnel and is included in the KSL data deliverable.

(d) The TA-50-RLWTF boiler was added to El as a new source in 2003. This boiler is owned and operated by a contractor and has been operated at LANL since mid-2000.

Originally planned as a temporary source, but current plans are to keep operating for several more years. Therefore, decision was made to include in LANL's annual EI. Fuel use has not been tracked monthly. For 2004, the total gas use for the year (Dec. 04' - Dec. 03') was taken and used to calculate emissions.

(e) The 12-month rolling average includes all gas use from all boilers listed in this spreadsheet. Boilers not included in this report due to their large size or design are TA-21 boilers \& powerplant boilers at TA-3. A gas use limit of $870 \mathrm{MMsct} / \mathrm{yr}, 12$-month rolling average is a permit limit in Section 2.4 of the LANL operating permit.

(f) The non-metered boiler pool capacity is the sum of all active non-metered boilers design ratings in MMBTU. In 2004, the TA-16 boilers were added to the boiler pool. This increased the boiler pool from 249.4 to $262.1 \mathrm{MMBtu} / \mathrm{Hr}$. This number is used to estimate the gas use rate (total non-metered gas use divided by the non-metered boiler pool capacity number).

(g) The non-metered boilers gas use section provides estimates of gas use for each boiler. This is calculated using the non-metered gas rate, as discussed in reference (f). The individual boiler design rating is mutiplied by the gas use rate to provide the estimated gas used per reporting period (in MMSCF).

(h) NMED List of Insignificant Activities (9/95), Item (3.) exempts fuel burning equipment which uses gaseous fuel, has a design rate less than or equal to 5 MMBTU/hr, and is used for heating buildings for personal comfort or for producing hot water for personal use.

(i) The design rate for boilers includes a correction for elevation. LANL is at approximately 7,500 feet above sea level. Corrections are made for atmospheric boilers using $4 \%$ reduction (derated) for each 1,000 feet above sea level $(4 \% \times 7.5=30 \%)$. For forced draft and power burner boilers, the reduction is half that of atmospheric at $15 \%$. The correction is made using the boiler plate input rating minus the appropriate percentage. 
2004 Small Boilers Emissions by Boiler for Annual El Reporting (Tons/Year)

\begin{tabular}{|c|c|c|c|c|c|c|c|c|c|c|c|c|}
\hline $\begin{array}{l}\text { Pollutant } \\
\text { Criteria }\end{array}$ & $\begin{array}{c}\text { AIRS } 015 \\
\text { TA-48-1 } \\
\text { BS-1 } \\
\end{array}$ & $\begin{array}{c}\text { AIRS 016 } \\
\text { TA-48-1 } \\
\text { BS-2 } \\
\end{array}$ & $\begin{array}{c}\text { AIRS } 017 \\
\text { TA-48-1 } \\
\text { BS-6 }\end{array}$ & $\begin{array}{c}\text { AIRS } 018 \\
\text { TA-53-365 } \\
\text { BHW-1 }\end{array}$ & $\begin{array}{c}\text { AJRS } 019 \\
\text { TA-53-365 } \\
\text { BHW-2 } \\
\end{array}$ & $\begin{array}{c}\text { AJRS } 020 \\
\text { TA-59-1 } \\
\text { BHW-1 }\end{array}$ & $\begin{array}{c}\text { AIRS } 021 \\
\text { TA-59-1 } \\
\text { BHW-2 }\end{array}$ & $\begin{array}{c}\text { AJRS } 024 \\
\text { TA-16 } \\
\text { Plant } 5 \\
\end{array}$ & $\begin{array}{r}\text { AIRS } 037 \\
\text { TA-55-6 } \\
\text { BHW-1B }\end{array}$ & $\begin{array}{c}\text { AIRS 038 } \\
\text { TA-55-6 } \\
\text { BHW-2B } \\
\end{array}$ & \begin{tabular}{|c|} 
AIRS 044 \\
TA-50-2 \\
BS-1 \\
\end{tabular} & $\begin{array}{c}\text { Total for } \\
\text { Small } \\
\text { Boilers }\end{array}$ \\
\hline NOx & 0.520 & 0.520 & 0.695 & 0.693 & 0.693 & 0.520 & 0.520 & 0.459 & 0.436 & 1.481 & 1.63E-02 & 6.551 \\
\hline sox & 0.003 & 0.003 & 0.004 & 0.004 & 0.004 & 0.003 & 0.003 & 0.007 & 0.002 & 0.006 & $9.76 \mathrm{E}-05$ & 0.041 \\
\hline PM & 0.039 & 0.039 & .053 & 0.053 & 0.053 & 0.039 & 0.039 & 0.094 & 0.045 & 0.152 & $.24 \mathrm{E}-03$ & 0.609 \\
\hline PM-10 & 0.039 & 0.039 & 0.053 & 0.053 & 0.053 & 0.039 & 0.039 & 0.094 & 0.045 & 0.152 & $4 \mathrm{E}-03$ & 0.609 \\
\hline PM-2.5 & 0.039 & 0.039 & 0.053 & 0.053 & 0.053 & 0.039 & 0.039 & 0.094 & 0.045 & 0.152 & $4 \mathrm{E}-03$ & 0.609 \\
\hline $\mathrm{CO}$ & 0.437 & 0.436 & 0.584 & 0.582 & 0.582 & 0.436 & 0.436 & 0.459 & 0.121 & 0.410 & $7 \mathrm{E}-02$ & 4.497 \\
\hline VOC & 0.029 & 0.029 & 0.038 & 0.038 & 0.038 & 0.029 & 0.029 & 0.068 & 0.019 & 0.064 & 8.95E-04 & 0.381 \\
\hline \multicolumn{13}{|l|}{ HAP's } \\
\hline Arsenic & $1.04 \mathrm{E}-06$ & $1.04 \mathrm{E}-06$ & $1.39 \mathrm{E}-06$ & $1.39 \mathrm{E}-06$ & $1.39 \mathrm{E}-06$ & $1.04 \mathrm{E}-06$ & $1.04 \mathrm{E}-06$ & $2.47 \mathrm{E}-06$ & $6.31 \mathrm{E}-07$ & $2.15 \mathrm{E}-06$ & $3.25 \mathrm{E}-08$ & $1.36 \mathrm{E}-05$ \\
\hline Benzene & $1.09 \mathrm{E}-05$ & $1.09 \mathrm{E}-05$ & 1.46E-05 & $1.45 \mathrm{E}-05$ & $1.45 \mathrm{E}-05$ & $1.09 \mathrm{E}-05$ & $1.09 \mathrm{E}-05$ & $2.60 \mathrm{E}-05$ & $6.63 \mathrm{E}-06$ & $2.25 \mathrm{E}-05$ & $3.42 E-07$ & $1.43 \mathrm{E}-04$ \\
\hline BE & $24 \mathrm{E}-08$ & $23 \mathrm{E}-08$ & $E-08$ & E-08 & $1 \mathrm{E}-08$ & $6.23 \mathrm{E}-08$ & $6.23 \mathrm{E}-08$ & $8 \mathrm{E}-07$ & $3.79 \mathrm{E}-08$ & $1.29 \mathrm{E}-07$ & $1.95 \mathrm{E}-09$ & $8.16 \mathrm{E}-07$ \\
\hline Cadmium & $72 \mathrm{E}-06$ & 72E-06 & E-06 & $62 \mathrm{E}-06$ & $7.62 \mathrm{E}-06$ & $5.72 \mathrm{E}-06$ & $5.72 \mathrm{E}-06$ & E-05 & $3.47 \mathrm{E}-06$ & $1.18 \mathrm{E}-05$ & E-07 & $7.48 \mathrm{E}-05$ \\
\hline Chromium & $7.28 \mathrm{E}-06$ & $7.27 \mathrm{E}-06$ & $9.73 \mathrm{E}-06$ & $9.70 \mathrm{E}-06$ & $9.70 \mathrm{E}-06$ & $7.27 \mathrm{E}-06$ & 7.27E-06 & $3 \mathrm{E}-05$ & $4.42 \mathrm{E}-06$ & $1.50 \mathrm{E}-05$ & $2.28 \mathrm{E}-07$ & $9.52 \mathrm{E}-05$ \\
\hline Cobalt & $4.37 \mathrm{E}-07$ & $4.36 \mathrm{E}-07$ & $5.84 \mathrm{E}-07$ & $5.82 \mathrm{E}-07$ & $5.82 \mathrm{E}-07$ & $4.36 \mathrm{E}-07$ & $4.36 \mathrm{E}-07$ & $1.04 \mathrm{E}-06$ & $2.65 \mathrm{E}-07$ & $9.01 E-07$ & $1.37 \mathrm{E}-08$ & $5.71 \mathrm{E}-06$ \\
\hline Dichlorobenzene & $6.24 \mathrm{E}-06$ & 6.23E-06 & $8.34 \mathrm{E}-06$ & 8.31E-06 & 8.31E-06 & $6.23 \mathrm{E}-06$ & $6.23 \mathrm{E}-06$ & $1.48 \mathrm{E}-05$ & $3.79 \mathrm{E}-06$ & $1.29 \mathrm{E}-05$ & 1.95E-07 & $8.16 \mathrm{E}-05$ \\
\hline Formaldehyde & $3.90 \mathrm{E}-04$ & $3.90 \mathrm{E}-04$ & $5.21 \mathrm{E}-04$ & $5.20 \mathrm{E}-04$ & $5.20 \mathrm{E}-04$ & $3.90 \mathrm{E}-04$ & $3.90 \mathrm{E}-04$ & $9.28 \mathrm{E}-04$ & $2.37 \mathrm{E}-04$ & $8.05 \mathrm{E}-04$ & 1.22E-05 & $5.10 \mathrm{E}-03$ \\
\hline Hexane & $9.35 \mathrm{E}-03$ & $9.35 \mathrm{E}-03$ & $1.25 \mathrm{E}-02$ & $1.25 \mathrm{E}-02$ & $1.25 \mathrm{E}-02$ & $9.35 \mathrm{E}-03$ & $9.35 \mathrm{E}-03$ & $2.23 \mathrm{E}-02$ & $5.68 \mathrm{E}-03$ & $1.93 \mathrm{E}-02$ & $2.93 \mathrm{E}-04$ & $1.22 \mathrm{E}-01$ \\
\hline Lead & $2.60 \mathrm{E}-06$ & 2.60E-06 & $3.48 \mathrm{E}-06$ & $3.46 \mathrm{E}-06$ & $3.46 \mathrm{E}-06$ & $2.60 \mathrm{E}-06$ & $2.60 \mathrm{E}-06$ & $6.18 \mathrm{E}-06$ & $1.58 \mathrm{E}-06$ & 5.37E-06 & $8.14 \mathrm{E}-08$ & $3.40 \mathrm{E}-05$ \\
\hline Mangenese & 1.97E-06 & 1.97E-06 & & 2.63E-06 & & & $1.97 \mathrm{E}-06$ & $4.70 \mathrm{E}-06$ & $1.20 \mathrm{E}-06$ & $4.08 E-06$ & $8 \mathrm{E}-08$ & $2.58 \mathrm{E}-05$ \\
\hline & 35E-06 & 1.35E-06 & $=-06$ & E-06 & $\overline{0 E-06}$ & $5 \mathrm{E}-06$ & 1.35E-06 & $3.22 \mathrm{E}-06$ & 8.21E-07 & $2.79 \mathrm{E}-06$ & $4.23 \mathrm{E}-08$ & $1.77 \mathrm{E}-05$ \\
\hline Napthalene & E-06 & 3.17E-06 & & & E-06 & $E-06$ & $7 \mathrm{E}-06$ & 7.54E-06 & 1.93E-06 & 6.55E-06 & E-08 & $4.15 \mathrm{E}-05$ \\
\hline Nickel & $09 \mathrm{E}-05$ & $1.09 \mathrm{E}-05$ & $1.46 \mathrm{E}-05$ & $1.45 \mathrm{E}-05$ & $1.45 \mathrm{E}-05$ & E-05 & E-05 & E-05 & $3 E-06$ & $2.25 \mathrm{E}-05$ & -07 & $1.43 \mathrm{E}-04$ \\
\hline POM & $4.57 \mathrm{E}-07$ & $4.57 \mathrm{E}-07$ & $6.12 \mathrm{E}-07$ & $6.10 \mathrm{E}-07$ & $6.10 \mathrm{E}-07$ & $4.57 E-07$ & $4.57 \mathrm{E}-07$ & $1.09 \mathrm{E}-06$ & $2.78 \mathrm{E}-07$ & $9.44 \mathrm{E}-07$ & $1.43 \mathrm{E}-08$ & $5.98 \mathrm{E}-06$ \\
\hline Selenium & $1.25 \mathrm{E}-07$ & $1.25 \mathrm{E}-07$ & $1.67 \mathrm{E}-07$ & $1.66 \mathrm{E}-07$ & $1.66 \mathrm{E}-07$ & $1.25 \mathrm{E}-07$ & $1.25 \mathrm{E}-07$ & $2.97 \mathrm{E}-07$ & $7.58 \mathrm{E}-08$ & $2.58 \mathrm{E}-07$ & $3.91 \mathrm{E}-09$ & $1.63 \mathrm{E}-06$ \\
\hline Toluene & 1.77E-05 & 1.77E-05 & $2.36 \mathrm{E}-05$ & $2.36 \mathrm{E}-05$ & $2.36 \mathrm{E}-05$ & 1.77E-05 & 1.77E-05 & $4.20 \mathrm{E}-05$ & 1.07E-05 & 3.65E-05 & 5.53E-07 & 2.31E-04 \\
\hline TOTAL HAPS/Unit & $9.81 E-03$ & $9.81 \mathrm{E}-03$ & $1.31 \mathrm{E}-02$ & $1.31 E-02$ & 1.31E-02 & $9.81 \mathrm{E}-03$ & $9.81 \mathrm{E}-03$ & $2.34 \mathrm{E}-02$ & $5.96 \mathrm{E}-03$ & $2.03 \mathrm{E}-02$ & $3.07 \mathrm{E}-04$ & 0.13 \\
\hline
\end{tabular}




\section{TA-52 Paper Shredder}

\begin{tabular}{|l|c|}
\cline { 2 - 2 } \multicolumn{1}{c|}{} & Data Entry \\
\hline Month & $\begin{array}{c}\text { Boxes } \\
\text { Shredded }\end{array}$ \\
\hline January & 95 \\
\hline February & 779 \\
\hline March & 1022 \\
\hline April & 1075 \\
\hline May & 353 \\
\hline June & 569 \\
\hline 6 mo. Total & 3,893 \\
\hline
\end{tabular}

\begin{tabular}{|l|c|}
\cline { 2 - 2 } \multicolumn{1}{c|}{} & Data Entry \\
\hline \multicolumn{1}{c|}{ Month } & $\begin{array}{c}\text { Boxes } \\
\text { Shredded }\end{array}$ \\
\hline July & 25 \\
\hline August & 0 \\
\hline September & \\
\hline October & \\
\hline November & \\
\hline December & \\
\hline 6 mo. Total: & 25 \\
\hline
\end{tabular}

The Paper Shredder was taken off line in July 2004

Annual Boxes (2004):

3,918

Maximum Annual emission rate is 13 tons of Total Suspended Particulate (TSP) per year.

\begin{tabular}{|c|c|c|c|c|c|c|c|c|c|}
\hline Emissions & $\begin{array}{c}\text { Boxes } \\
\text { Shredded }\end{array}$ & $\begin{array}{l}\text { Weight } \\
\text { per box }\end{array}$ & $\begin{array}{l}\text { Amount }^{3} \\
\text { Processed } \\
\text { (pounds) }\end{array}$ & $\begin{array}{c}\text { Emission }{ }^{2} \\
\text { Factor }\end{array}$ & $\begin{array}{l}\text { Control }^{4} \\
\text { Efficiency } \\
\text { (Cyclone) }\end{array}$ & $\begin{array}{l}\text { Control } \\
\text { Efficiency } \\
\text { (Bag } \\
\text { house) }\end{array}$ & $\begin{array}{c}\text { TSP } \\
\text { Emissions } \\
\text { (pounds) }\end{array}$ & $\begin{array}{l}\text { PM-10 } \\
\text { (pounds) }\end{array}$ & $\begin{array}{c}\text { PM-2.5 } \\
\text { (pounds) }\end{array}$ \\
\hline Annual & 3,918 & 60 & 235,080 & $1 \%$ & $90 \%$ & $99 \%$ & 2.35 & 2.35 & 2.35 \\
\hline January - June & 3,893 & 60 & 233,580 & $1 \%$ & $90 \%$ & $99 \%$ & 2.34 & 2.34 & 2.34 \\
\hline July - December & 25 & 60 & 1,500 & $1 \%$ & $90 \%$ & $99 \%$ & 0.02 & 0.02 & 0.02 \\
\hline
\end{tabular}

Reterence

1. Estimated box weight is 60

pounds. Information provided by

shredding operations manager.

2. Emission Factor obtained from SEM, the shredder manufacturer.

\begin{tabular}{|l|l|}
$\begin{array}{l}\text { 3. Information provided by } \\
\text { the shredder operator } \\
\text { (KSL). }\end{array}$ & $\begin{array}{l}\text { 4. Information on control } \\
\text { equipment efficiencies is } \\
\text { based on engineering } \\
\text { judgment. }\end{array}$ \\
\hline
\end{tabular}


Degreasers TA-55

2004 Usage Data

\begin{tabular}{|c|c|c|c|c|c|}
\hline Date Measured & $\begin{array}{c}\text { Tnitial Solvent Level } \\
\text { (inches) }\end{array}$ & $\begin{array}{c}\text { Volume Added } \\
\text { (liters) }\end{array}$ & $\begin{array}{l}\text { Level Added } \\
\text { (inches) }\end{array}$ & $\begin{array}{c}\text { Volume Removed } \\
\text { (liters) }\end{array}$ & $\begin{array}{l}\text { Level Removed } \\
\text { (inches) }\end{array}$ \\
\hline Jan-05-2004 & 7.3 & 0 & 0 & 0 & 0 \\
\hline Feb-02-2004 & 7.1 & 0 & 0 & 0 & 0 \\
\hline Mar-01-2004 & 6.8 & 0 & 0 & 0 & 0 \\
\hline Apr-01-2004 & 6.5 & 0 & 0 & 0 & $\mathbf{0}$ \\
\hline May-03-2004 & 6.3 & 2.35 & 1.2 & 0 & 0 \\
\hline Jun-01-2004 & 6.9 & 0 & 0 & 0 & 0 \\
\hline Jun-09-2004 & 6.9 & 1.17 & 0.6 & 0 & 0 \\
\hline Jul-01-2004 & 7.3 & 0 & 0 & 0 & 0 \\
\hline Aug-02-2004 & 7 & 0 & 0 & 0 & 0 \\
\hline Sep-01-2004 & 6.8 & 0 & 0 & 0 & 0 \\
\hline Oct-04-2004 & 6.4 & 0 & 0 & 0 & 0 \\
\hline Nov-01-2004 & 6 & 0 & 0 & 0 & 0 \\
\hline Dec-01-2004 & 5.9 & 0 & 0 & 0 & 0 \\
\hline Dec-15-2004 & 5.9 & 3.14 & 1.6 & 0 & 0 \\
\hline Jan-03-2005 & 7.4 & 0 & 0 & 0 & 0 \\
\hline TOTAL & & 6.66 & & 0 & \\
\hline
\end{tabular}

Conversions
\begin{tabular}{|l|r|}
\hline $\mathrm{Kg} / \mathrm{L}$ & 1.463 \\
\hline $\mathrm{Lb} / \mathrm{Kg}$ & 2.21 \\
\hline $\mathrm{lb} / \mathrm{ton}$ & 2000 \\
\hline
\end{tabular}

Emissions $=\quad($ Vol Added $-\mathrm{L})-($ Vol Removed $-\mathrm{L}) \times(1.463 \mathrm{Kg} / \mathrm{L}) \times(2.21 \mathrm{Lg} / \mathrm{Kg})=$

The Usage information for UT Bath" degreaser from "Jan-01-2004" through "Jan-24-2005". "

Type: Cold Batch

TA: 55

Bldg:

Solvent: Trichloroethylene 


\begin{tabular}{|c|c|c|c|c|c|c|c|c|}
\hline & Data Entry & & & Data Entry & & & & \\
\hline \begin{tabular}{|c} 
Month \\
\end{tabular} & $\begin{array}{l}\text { Boxes }^{(e)} \\
\text { Shredded }\end{array}$ & $\begin{array}{c}\text { 12-Month } \\
\text { Rolling } \\
\text { Total }\end{array}$ & Month & $\begin{array}{l}\text { Boxes }^{(e)} \\
\text { Shredded }\end{array}$ & $\begin{array}{c}\text { 12-Month } \\
\text { Rolling } \\
\text { Total }\end{array}$ & & & \\
\hline January & & & July & & & & & \\
\hline February & & & August & 58 & 58 & Data Disinte & rator Started & up in August 2004 \\
\hline March & & & September & 903 & 961 & & & \\
\hline April & & & October & 717 & 1678 & & & \\
\hline May & & & November & 451 & 2129 & & & \\
\hline June & & & December & 237 & 2366 & & & \\
\hline 6 mo. Total & 0 & & 6 mo. Total: & 2,366 & & & & \\
\hline Annual Boxes & (2004): & 2,366 & & & & & & \\
\hline Maximum Annual em & ission rate is & $\begin{array}{l}9.9 \text { tpy or } 2 . \\
9.9 \text { tpy or } 2 \text {. }\end{array}$ & $\begin{array}{l}3 \mathrm{lb} / \mathrm{hr} \text { of Tota } \\
3 \mathrm{lb} / \mathrm{hr} \text { of Part }\end{array}$ & $\begin{array}{l}\text { Suspended } P \\
\text { culate Matter }\end{array}$ & $\begin{array}{l}\text { articulate (TS } \\
\text { e10 } 10 \mathrm{~m} \text { (PM-1 }\end{array}$ & $\begin{array}{l}\text { SP) per year. } \\
\text { 0) per year. }\end{array}$ & & \\
\hline Emission Calcula & tions & & & & & & & \\
\hline & $\begin{array}{l}\text { Emission }^{(b)} \\
\text { Factor }\end{array}$ & $\begin{array}{c}\% \text { in }^{(e)} \\
\text { Exhaust }\end{array}$ & $\begin{array}{l}\text { Controf }^{(d)} \\
\text { Efficiency } \\
\text { (Cyclone) }\end{array}$ & $\begin{array}{l}\text { Control(d) }^{(\mathrm{e})} \\
\text { Efficiency } \\
\text { (Baghouse) }\end{array}$ & & & & \\
\hline $\begin{array}{l}\text { PM 2.5 } \\
\text { PM 10 }\end{array}$ & $15 \%$ & $15 \%$ & $0 \%$ & $95.0 \%$ & & Average B & X Weight ${ }^{(\text {(a) }}$ & \\
\hline TSP & $\begin{array}{l}15 \% \\
15 \%\end{array}$ & $90 \%$ & $75 \%$ & $95.0 \%$ & & & Pounds & \\
\hline & $\begin{array}{l}\text { Amount } \\
\text { Processed } \\
\text { (pounds) }\end{array}$ & $\begin{array}{c}\text { PM-2.5 } \\
\text { Emissions } \\
\text { (pounds) }\end{array}$ & $\begin{array}{c}\text { PM-2.5 } \\
\text { Emissions } \\
\text { (tons) }\end{array}$ & $\begin{array}{c}\text { PM-10 } \\
\text { Emissions } \\
\text { (pounds) }\end{array}$ & $\begin{array}{c}\text { PM-10 } \\
\text { Emissions } \\
\text { (tons) }\end{array}$ & $\begin{array}{c}\text { TSP } \\
\text { Emissions } \\
\text { (pounds) }\end{array}$ & $\begin{array}{c}\text { TSP } \\
\text { Emissions } \\
\text { (tons) }\end{array}$ & \\
\hline Annual & 106,470 & 119.8 & 0.060 & 179.7 & 0.090 & 199.6 & 0.100 & \\
\hline January - June & 0 & 0.0 & 0.000 & 0.0 & 0.000 & 0.0 & 0.000 & \\
\hline July - December & 106,470 & 119.8 & 0.060 & 179.7 & 0.090 & 199.6 & 0.100 & \\
\hline Reference & & & & & & & & \\
\hline $\begin{array}{l}\text { (a). Estimated } \\
\text { maximum box weight } \\
\text { is } 45 \text { pounds. } \\
\text { Information provided } \\
\text { by shredding } \\
\text { operations. Full box } \\
\text { weight of tightly } \\
\text { packed paper. }\end{array}$ & $\begin{array}{l}\text { (b). Emission } \\
\text { material shre } \\
\text { exhaust) obta } \\
\text { of the air han } \\
\text { Manufacturin } \\
\text { the construct }\end{array}$ & $\begin{array}{l}\text { Factor (perc } \\
\text { dded that will } \\
\text { ined from the } \\
\text { dling system, } \\
\text { Co. } 15 \% \text { is } \\
\text { on permit. }\end{array}$ & $\begin{array}{l}\text { entage of } \\
\text { enter into the } \\
\text { manufacturer } \\
\text { AGET } \\
\text { also listed in }\end{array}$ & \begin{tabular}{|l|} 
(c). \\
information \\
provided by \\
the shredding \\
operations \\
personnel.
\end{tabular} & $\begin{array}{l}\text { (d). Informat } \\
\text { efficiencies } \\
\text { manufacture } \\
\text { (SEM). Tho } \\
\text { extrapolated } \\
\text { Efficiencies } \\
95 \% \text { for the } \\
\text { construction } \\
\text { efficiency tal }\end{array}$ & $\begin{array}{l}\text { ion on contro } \\
\text { was provided } \\
\text { r of the Data } \\
\text { se values not } \\
\text { using manuf } \\
\text { of } 75 \% \text { for the } \\
\text { bag house ar } \\
\text { permit. (see } \\
\text { b for more inf }\end{array}$ & $\begin{array}{l}\text { equipment } \\
\text { y the } \\
\text { jisintegrator } \\
\text { given were } \\
\text { cturer data. } \\
\text { Cyclone and } \\
\text { listed in the } \\
\text { cyclone } \\
\text { ) }\end{array}$ & $\begin{array}{l}\text { (e). Manufacturer } \\
\text { provided info that the dust } \\
\text { into the exhaust would be } \\
\text { in the size range of } 5-20 \\
\text { um. Conservative } \\
\text { assumption that } 15 \% \text { is } \\
\text { PM2.5, and } 90 \% \text { is } \\
\text { PM10. }\end{array}$ \\
\hline
\end{tabular}


2004 TA-3 \& TA-15 Carpenter Shops

\begin{tabular}{|l|c|}
\hline \multicolumn{1}{|c|}{ TA-3 } & Data Entry \\
\hline \multirow{2}{*}{ Month } & Hours of Operation \\
\cline { 2 - 2 } & TA-3 \\
\hline January & 11 \\
\hline February & 10.5 \\
\hline March & 3.5 \\
\hline April & 12.5 \\
\hline May & 5.5 \\
\hline June & 6.5 \\
\hline 6 mo. Total & 49.50 \\
\hline
\end{tabular}

\begin{tabular}{|l|c|}
\hline & Data Entry \\
\hline \multirow{3}{*}{ Month } & Hours of Operation' \\
\cline { 2 - 2 } & TA-3 \\
\hline July & 3.7 \\
\hline August & 0 \\
\hline September & 0.5 \\
\hline October & 0.75 \\
\hline November & 7 \\
\hline December & 3.5 \\
\hline 6 mo. Total: & 15.45 \\
\hline
\end{tabular}

\begin{tabular}{|l|c|}
\hline \multicolumn{1}{|c|}{ TA-15 } & Data Entry \\
\hline \multirow{2}{*}{ Month } & Hours of Operation \\
\cline { 2 - 2 } & TA-15 \\
\hline January & 0 \\
\hline February & 0 \\
\hline March & 0 \\
\hline April & 0 \\
\hline May & 0 \\
\hline June & 0 \\
\hline 6 mo. Total & 0 \\
\hline
\end{tabular}

\begin{tabular}{|l|c|}
\hline & Data Entry \\
\hline \multirow{3}{*}{ Month } & Hours of Operation \\
\cline { 2 - 2 } & TA-15 \\
\hline July & 0 \\
\hline August & 0 \\
\hline September & 0 \\
\hline October & 0 \\
\hline November & 0 \\
\hline December & 0 \\
\hline 6 mo. Total: & 0 \\
\hline
\end{tabular}

Saws, drills, shaping and sanding equipment shall each operate at a maximum of 4368 hours per year.

\begin{tabular}{|l|}
\hline \multicolumn{1}{|c|}{ Reference } \\
\hline $\begin{array}{l}\text { 1. Based on information provided } \\
\text { monthly by the shop foreman } \\
\text { from each shop. }\end{array}$ \\
\hline
\end{tabular}

Allowable Emission Limits are:

monthly by the shop foreman

from each shop.

Reviewed By/Date: 
Carpenter Shop Emissions Calculations for 2004

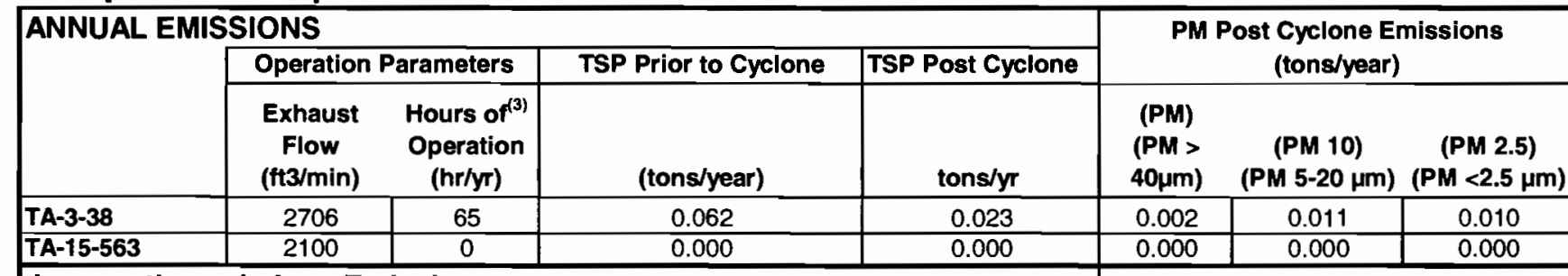

\begin{tabular}{|c|c|c|c|c|c|c|c|}
\hline & & & & & & & \\
\hline \multicolumn{5}{|c|}{ January through June Emissions } & \multirow{2}{*}{\multicolumn{3}{|c|}{$\begin{array}{c}\text { PM Post Cyclone Emissions } \\
\text { (tons) }\end{array}$}} \\
\hline & \multicolumn{2}{|c|}{ Operation Parameters } & \multirow{2}{*}{$\begin{array}{c}\text { TSP Prior to Cyclone } \\
\text { tons }\end{array}$} & \multirow{2}{*}{$\begin{array}{c}\text { TSP Post Cyclone } \\
\text { tons } \\
\end{array}$} & & & \\
\hline & $\begin{array}{l}\text { Exhaust } \\
\text { Flow } \\
\text { (ft3/min) }\end{array}$ & $\begin{array}{l}\text { Hours of }^{(3)} \\
\text { Operation } \\
\text { (hr/period) }\end{array}$ & & & $\begin{array}{l}\text { (PM) } \\
(\mathrm{PM}> \\
40 \mu \mathrm{m})\end{array}$ & $\begin{array}{c}\text { (PM 10) } \\
\text { (PM 5-20 } \mu \mathrm{m})\end{array}$ & $\begin{array}{c}\text { (PM 2.5) } \\
(\mathrm{PM}<2.5 \mu \mathrm{m})\end{array}$ \\
\hline TA-3-38 & 2706 & 50 & 0.047 & 0.017 & 0.001 & 0.008 & 0.008 \\
\hline TA-15-563 & 2100 & 0 & 0.000 & 0.000 & 0.000 & 0.000 & 0.000 \\
\hline \multicolumn{5}{|c|}{ July through December Emissions } & \multicolumn{3}{|c|}{ PM Post Cyclone Emissions } \\
\hline
\end{tabular}

July through December Emissions

PM Post Cyclone Emissions

\begin{tabular}{|c|c|c|c|c|c|c|c|}
\hline & \multicolumn{2}{|c|}{ Operation Parameters } & \multirow{2}{*}{$\begin{array}{c}\text { TSP Prior to Cyclone } \\
\text { tons } \\
\end{array}$} & \multirow{2}{*}{$\begin{array}{c}\text { TSP Post Cyclone } \\
\text { tons } \\
\end{array}$} & \multicolumn{3}{|c|}{ (tons) } \\
\hline & $\begin{array}{c}\text { Exhaust }^{(1)} \\
\text { Flow } \\
\text { (ft3/min) }\end{array}$ & $\begin{array}{l}\text { Hours of }^{(3)} \\
\text { Operation } \\
\text { (hr/period) }\end{array}$ & & & $\begin{array}{l}\text { (PM) } \\
(P M> \\
40 \mu m)\end{array}$ & $\begin{array}{c}\text { (PM 10) } \\
\text { (PM 5-20 } \mu \mathrm{m})\end{array}$ & $\begin{array}{c}\text { (PM 2.5) } \\
(\mathrm{PM}<2.5 \mu \mathrm{m})\end{array}$ \\
\hline TA-3-38 & 2706 & 15 & 0.015 & 0.005 & 0.000 & 0.003 & 0.002 \\
\hline TA-15-563 & 2100 & 0 & 0.000 & 0.000 & 0.000 & 0.000 & 0.000 \\
\hline
\end{tabular}

Conversions:
\begin{tabular}{|c|c|c|c|}
\hline $\mathrm{lb} /$ ton & Ib/grain & $\mathrm{min} / \mathrm{hr}$ & ton/lb \\
\hline 2000 & 0.000142857 & 60 & 0.0005 \\
\hline
\end{tabular}

References: 1.) Exhaust Rate calculated by Victor Martinez.

2.) Emission Factor obtained from AP-42, Section 10.4

Woodworking Waste Collection Operations, post cyclone emissions, Table 10.4.1, February 1980.

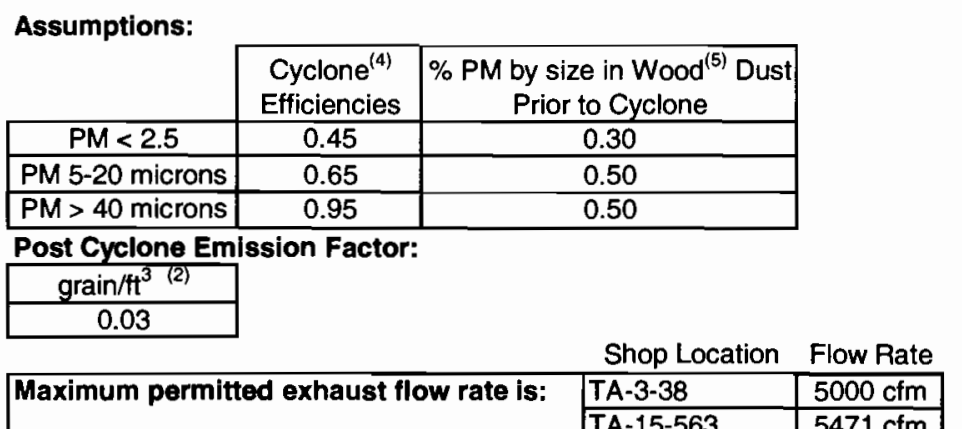

3.) Based on information provided monthly by the shop

oreman.

4.) K. Wark \& C.F. Warner, Air Pollution - Its Origin and

Control, Table 5-9, pg 186 (1976).

5.) Emissions Inventory Improvement Program (EIIP)

Uncontrolled Emission Factor Listing for Criteria Air

Pollutants, Volume II: Chapter 14, July 2001 And AP-42

Appendix B, Section 10.5 Woodworking Waste Collection

Operations: Belt Sander Hood Exhaust Cyclone.

Reviewed By/Date: 
2004 Generator Emissions

\begin{tabular}{|c|c|c|c|c|c|c|c|c|c|c|c|c|c|c|}
\hline \multirow[b]{2}{*}{$T A$} & \multirow[b]{2}{*}{$B / d g$} & \multirow[b]{2}{*}{ Manufacturer } & \multirow[b]{2}{*}{$K W$} & \multirow[b]{2}{*}{ Fuel Type } & \multicolumn{5}{|c|}{ First 6 Month Readings 2004} & \multicolumn{5}{|c|}{ Second 6 Month Readings 2004} \\
\hline & & & & & $\begin{array}{c}\text { 6honth } \\
\text { Reading } \\
\text { Date }\end{array}$ & Reading & $\begin{array}{c}\text { Hours } \\
\text { Run }\end{array}$ & $\begin{array}{c}\text { Days } \\
\text { between } \\
\text { readings }\end{array}$ & $\begin{array}{c}6 \mathrm{mth} \\
\text { Prorated } \\
\text { Hours }\end{array}$ & $\begin{array}{c}\text { T2 Month } \\
\text { Reading } \\
\text { Date }\end{array}$ & Reading & $\begin{array}{c}\text { Hours } \\
\text { Run }\end{array}$ & $\begin{array}{l}\text { Days } b / t \\
\text { readings }\end{array}$ & $\begin{array}{c}6 \mathrm{mth} \\
\text { Prorated } \\
\text { Hours }\end{array}$ \\
\hline 3 & 40 & Onan Sons & 150 & Diesel & $05 / 01 / 2004$ & 231 & 11.6 & 180 & 11.6 & $12 / 01 / 2004$ & 545.0 & 314 & 210 & 269.1 \\
\hline 3 & 223 & Onan Sons & 45 & Nat. Gas & $05 / 01 / 2004$ & 446 & 7.8 & 180 & 7.8 & $12 / 01 / 2004$ & 452.1 & 6.1 & 210 & 5.2 \\
\hline 3 & 1404 & Curnmins & 1250 & Diesel & $04 / 01 / 2004$ & 60.5 & 22.5 & 120 & 33.8 & $11 / 01 / 2004$ & 79.0 & 18.5 & 210 & 15.9 \\
\hline 3 & 440 & Cummins & 150 & Diesel & $05 / 01 / 2004$ & 92.8 & 6.6 & 180 & 6.6 & $12 / 01 / 2004$ & 98.0 & 5.2 & 210 & 4.5 \\
\hline 3 & 440 & Cummins & 500 & Diesel & $05 / 01 / 2004$ & 38 & 6.8 & 180 & 6.8 & $12 / 01 / 2004$ & 42.9 & 4.9 & 210 & 4.2 \\
\hline 3 & 1076 & Cummins & 35 & Diesel & N/R & - & - & - & 0.0 & $01 / 01 / 2005$ & 44.5 & New & New & 44.5 \\
\hline 3 & 1498 & Caterpillar & 600 & Diesel & $05 / 01 / 2004$ & 266 & 6 & 180 & 6.0 & $12 / 01 / 2004$ & 269.0 & 3 & 210 & 2.6 \\
\hline 3 & 2322 & Onan Sons & 80 & Diesel & $05 / 01 / 2004$ & 20.2 & 20.2 & 180 & 20.2 & $12 / 01 / 2004$ & 56.8 & 36.6 & 210 & 31.4 \\
\hline 16 & 205 & Onan Sons & 250 & Diesel & $05 / 01 / 2004$ & 983.5 & 20.7 & 180 & 20.7 & $11 / 01 / 2004$ & 1008.7 & 25.2 & 180 & 25.2 \\
\hline 16 & 980 & Cummins & 1100 & Diesel & N/R & - & - & - & 0.0 & $12 / 01 / 2004$ & 10.4 & New & New & 10.4 \\
\hline 16 & 1374 & Onan Sons & 60 & Nat. Gas & $05 / 01 / 2004$ & 815.2 & 43.1 & 180 & 43.1 & $12 / 01 / 2004$ & 865.2 & 50 & 210 & 42.9 \\
\hline 18 & 31 & Onan Sons & 275 & Diesel & $05 / 01 / 2004$ & 140.5 & 6 & 180 & 6.0 & $12 / 01 / 2004$ & 147.6 & 7.1 & 210 & 6.1 \\
\hline 21 & 155 & Onan Sons & 750 & Diesel & $05 / 01 / 2004$ & 822.6 & 11.3 & 180 & 11.3 & $12 / 01 / 2004$ & 825.6 & 3 & 210 & 2.6 \\
\hline 21 & 257 & Onan Sons & 20 & Diesel & $05 / 01 / 2004$ & 189 & 0 & 180 & 0.0 & $12 / 01 / 2004$ & 189.0 & 0 & 210 & 0.0 \\
\hline 21 & 357 & Caterpillar & 125 & Diesel & $05 / 01 / 2004$ & 441 & 10.8 & 180 & 10.8 & $12 / 01 / 2004$ & 445.0 & 4 & 210 & 3.4 \\
\hline 21 & 1002 & Onan Sons & 175 & Diesel & $05 / 01 / 2004$ & 2876.2 & 5.2 & 180 & 5.2 & $11 / 01 / 2004$ & 2878.2 & 2 & 180 & 2.0 \\
\hline 21 & 1002 & Onan Sons & 350 & Diesel & $05 / 01 / 2004$ & 1695.1 & 15.1 & 180 & 15.1 & $12 / 01 / 2004$ & 1770.9 & 75.8 & 210 & 65.0 \\
\hline 21 & 1002 & Cummins & 150 & Diesel & $05 / 01 / 2004$ & 1016 & 2.1 & 180 & 2.1 & $12 / 01 / 2004$ & 1072.8 & 56.8 & 210 & 48.7 \\
\hline 33 & 87 & Kohler & 30 & Diesel & $05 / 01 / 2004$ & 822.8 & 439.9 & 180 & 439.9 & $12 / 01 / 2004$ & 840.5 & 17.7 & 210 & 15.2 \\
\hline 33 & 151 & Caterpillar & 225 & Diesel & $05 / 01 / 2004$ & - & - & $\cdot$ & 0.0 & $12 / 01 / 2004$ & 2944.0 & 0 & New & 0.0 \\
\hline 33 & 208 & Kohler & 1600 & Diesel & $04 / 01 / 2004$ & 4.9 & 0 & 120 & 0.0 & $12 / 01 / 2004$ & 4.9 & 0 & 240 & 0.0 \\
\hline 33 & Point & Onan Sons & 80 & Diesel & $05 / 01 / 2004$ & 7643.1 & 0 & 150 & 0.0 & $12 / 01 / 2004$ & 7643.1 & 0 & 210 & 0.0 \\
\hline 35 & 2 & Onan Sons & 100 & Diesel & $05 / 01 / 2004$ & 93.5 & 3.7 & 150 & 4.4 & $01 / 01 / 2005$ & 95.3 & 1.8 & 240 & 1.4 \\
\hline 43 & 1 & Cummins & 50 & Diesel & $05 / 01 / 2004$ & 331.6 & 7.9 & 180 & 7.9 & $12 / 01 / 2004$ & 344.6 & 13 & 210 & 11.1 \\
\hline 43 & 1 & Onan Sons & 150 & Diesel & $05 / 01 / 2004$ & 436.8 & 29.6 & 180 & 29.6 & $12 / 01 / 2004$ & 455.3 & 18.5 & 210 & 15.9 \\
\hline 46 & 335 & Onan Sons & 300 & Diesel & $05 / 01 / 2004$ & 651.6 & 24.9 & 180 & 24.9 & $12 / 01 / 2004$ & 717.3 & 65.7 & 210 & 56.3 \\
\hline 48 & 45 & Onan Sons & 125 & Diesel & $05 / 01 / 2004$ & 322 & 5.9 & 180 & 5.9 & $12 / 01 / 2004$ & 328.5 & 6.5 & 210 & 5.6 \\
\hline 50 & 37 & Cummins & 500 & Diesel & $05 / 01 / 2004$ & 458.3 & 1.4 & 180 & 1.4 & $12 / 01 / 2004$ & 463.8 & 5.5 & 210 & 4.7 \\
\hline 50 & 184 & Onan Sons & 60 & Nat. Gas & $04 / 01 / 2004$ & 809 & 39.5 & 150 & 47.4 & $01 / 01 / 2005$ & 49.5 & 49.5 & New & 49.5 \\
\hline 50 & 188 & Onan Sons & 1250 & \begin{tabular}{|l|} 
Diesel \\
\end{tabular} & $05 / 01 / 2004$ & 125.2 & 7.3 & 180 & 7.3 & $12 / 01 / 2004$ & 131.2 & 6 & 210 & 5.1 \\
\hline 53 & 1 & Onan Sons & 60 & Nat. Gas & $05 / 01 / 2004$ & 917.1 & 44.6 & 180 & 44.6 & $12 / 01 / 2004$ & 971.1 & 54 & 210 & 46.3 \\
\hline 53 & 2 & Kato Eng. & 50 & Diesel & $05 / 01 / 2004$ & 182.7 & 6.4 & 180 & 6.4 & $12 / 01 / 2004$ & 189.0 & 6.3 & 210 & 5.4 \\
\hline 53 & $M$ & Cummins & 60 & Diesel & $04 / 01 / 2004$ & 4440 & 0 & 180 & 0.0 & $12 / 01 / 2004$ & 4440.0 & 0 & 240 & 0.0 \\
\hline 53 & $M$ & Onan Sons & 12.5 & Nat. Gas & $04 / 01 / 2004$ & 581.5 & 0 & 180 & 0.0 & $12 / 01 / 2004$ & 581.5 & 0 & 240 & 0.0 \\
\hline 54 & 412 & Olympian & 500 & Diesel & $05 / 01 / 2004$ & 223.7 & 9.3 & 150 & 11.2 & $12 / 01 / 2004$ & 242.6 & 18.9 & 210 & 16.2 \\
\hline 55 & 5 & & 100 & Nat. Gas & $05 / 01 / 2004$ & 34 & 9.2 & 180 & 9.2 & $12 / 01 / 2004$ & 44.7 & 10.7 & 210 & 9.2 \\
\hline
\end{tabular}


2004 Generator Emissions

\begin{tabular}{|c|c|c|c|c|c|c|c|c|c|c|c|c|c|c|}
\hline 55 & 8 & Detroit & 600 & Diesel & $05 / 01 / 2004$ & 743 & 8.4 & 150 & 10.1 & $12 / 01 / 2004$ & 760.6 & 17.6 & 210 & 15.1 \\
\hline 55 & 28 & Onan Sons & 40 & Diesel & $05 / 01 / 2004$ & 24 & 6.3 & 180 & 6.3 & $12 / 01 / 2004$ & 31.4 & 7.4 & 210 & 6.3 \\
\hline 55 & 47 & Onan Sons & 200 & Diesel & $05 / 01 / 2004$ & 461 & 4.5 & 180 & 4.5 & $12 / 01 / 2004$ & 480.6 & 19.6 & 210 & 16.8 \\
\hline 55 & 142 & Cummins & 400 & Diesel & $05 / 01 / 2004$ & 24.8 & 0.9 & 150 & 1.1 & $12 / 01 / 2004$ & 24.8 & 0 & 210 & 0.0 \\
\hline 59 & 1 & Allis Chalmers & 90 & Diesel & $05 / 01 / 2004$ & 719.5 & 7.1 & 180 & 7.1 & $12 / 01 / 2004$ & 725.5 & 6 & 210 & 5.1 \\
\hline 61 & 23 & Murphy & 20 & Diesel & $05 / 01 / 2004$ & 557 & 0 & 180 & 0.0 & $12 / 01 / 2004$ & 569.9 & 12.9 & 210 & 11.1 \\
\hline 64 & 1 & Onan Sons & 250 & Diesel & $05 / 01 / 2004$ & 111.6 & 6 & 180 & 6.0 & $12 / 01 / 2004$ & 114.6 & 3 & 210 & 2.6 \\
\hline 69 & 33 & Cummins & 1250 & Diesel & $\mathrm{N} / \mathrm{R}$ & $\cdot$ & - & - & 0.0 & $12 / 01 / 2004$ & 16.5 & New & New & 16.5 \\
\hline
\end{tabular}

44 Generators in use

N/R = Not Read

First half average hours per unit

\begin{tabular}{|l|l}
\hline 20.1 & Second half average hours per unit
\end{tabular}

20.4

Annual Average of hours per unit

20.24

Reviewed by:

Date: 
2004 Generator Emissions

\begin{tabular}{|l|c|c|c|c|c|c|}
\hline EMISSION FACTORS & NOx & CO & SOx & PM & PM $_{10}$ & VOC \\
& lb/kw-hr & lb/kw-hr & lb/kw-hr & lb/kw-hr & lb/kw-hr & lb/kw-hr \\
\hline Large Diesel fired $^{(a)(b)}$ & 0.032 & 0.007 & 0.011 & 0.001 & 0.001 & 0.001 \\
\hline Small Diesel fired $^{(\mathrm{a})(\mathrm{c})}$ & 0.042 & 0.009 & 0.003 & 0.003 & 0.003 & 0.003 \\
\hline Natural Gas Fired $^{(\mathrm{d})}$ & 0.008 & 0.013 & $2.0 \mathrm{E}-06$ & $3.4 \mathrm{E}-05$ & $3.2 \mathrm{E}-05$ & $1.0 \mathrm{E}-04$ \\
\hline
\end{tabular}

(a) Emission factors from AP-42, Table 3.4-1

(b) AP-42, Fifth Edition, Table 3.3-1

(c) Emission factors from AP-42, July 2000, Table 3.2-1 Natural Gas Fired Reciprocating Engines. These

emission factors were used to reflect the same values as listed in the operating permit app.

References:

\begin{tabular}{|c|c|}
\hline 447 & $\begin{array}{l}447 \mathrm{kw} \text { is the size limit for determining large vs. small diesel fired } \\
\text { generator. This information was taken from the operating permit } \\
\text { application. }\end{array}$ \\
\hline \multicolumn{2}{|c|}{$\begin{array}{l}\text { (a) The AP-42 (fifth edition) emissions factor uses units of lb/hp-hr. There are } 1.341 \mathrm{hp}-\mathrm{hrs} \text { in a kwh. } \\
\text { Therefore, take pounds/hp-hr } \times 1.341 \mathrm{hp} \text {-hr/kwh to obtain the emission factor in lb/kwh. }\end{array}$} \\
\hline \multicolumn{2}{|c|}{$\begin{array}{l}\text { (b) Emission factors for large diesel fired boilers were taken from AP-42 (fifth edition) Tables 3.4-1, 3.4 } \\
2,3.4-3 \text {, and 3.4-4. }\end{array}$} \\
\hline \multicolumn{2}{|c|}{$\begin{array}{l}\text { (c) Emission factors for small diesel fired boilers were taken from AP-42 (fifth edition) Tables 3.3-1 ano } \\
\text { 3.3-2. }\end{array}$} \\
\hline \multicolumn{2}{|c|}{$\begin{array}{l}\text { (d) The AP-42 (fifth edition) emission factors for natural gas burning 4-stroke rich-burn engines (Table } \\
\text { 3.2-3) provides units of } \mathrm{lb} / \mathrm{MMBtu} \text {. There are } 3413 \mathrm{Btus} \text { in a kilowatt-hr }(\mathrm{kwh}) \text { or } 2.928 \times 10^{-4} \mathrm{kwh} \text { per } \\
\text { btu. Therefore, take } \mathrm{lb} / \mathrm{MMBtu} \times 3413 / 1 \times 10^{6} \text { or } \mathrm{lb} / \mathrm{MMbtu} / 10^{6} / 2.928 \times 10^{-4} \text { to obtain the emissions } \\
\text { factor in } \mathrm{lb} / \mathrm{kwh} \text {. }\end{array}$} \\
\hline
\end{tabular}


2004 Generator Emissions

First 6 Month Emissions of 2004

Second 6 Month Emissions of 2004

\begin{tabular}{|c|c|c|c|c|c|c|c|c|c|c|c|c|}
\hline \multirow[b]{2}{*}{ Location } & \multicolumn{6}{|c|}{ First 6 Month Emissions of 2004} & \multicolumn{6}{|c|}{ Second 6 Month Emissions of 2004} \\
\hline & $\begin{array}{c}\text { NOx } \\
\text { (lb/yr) }\end{array}$ & $\begin{array}{c}C O \\
(I b / y r)\end{array}$ & $\begin{array}{c}\text { SOx } \\
\text { (lb/yr) }\end{array}$ & $\begin{array}{c}P M \\
\text { (lb/yr) }\end{array}$ & $\begin{array}{c}\text { VOC } \\
(l b / y r)\end{array}$ & $\begin{array}{l}\text { HAPs } \\
\text { (Ib/yr) }\end{array}$ & $\begin{array}{c}\text { NOx } \\
(\mathbf{l b} / \mathbf{y} r)\end{array}$ & $\begin{array}{c}c O \\
(\mid b / y r)\end{array}$ & $\begin{array}{c}\text { SOx } \\
(\mid l b / y r)\end{array}$ & $\begin{array}{c}P M \\
(I b / y r)\end{array}$ & $\begin{array}{c}\text { VOC } \\
\text { (lb/yr) }\end{array}$ & $\begin{array}{l}\text { HAPS } \\
\text { (Ib/yr) }\end{array}$ \\
\hline $3-40$ & 72.3 & 15.6 & 4.8 & 5.1 & 5.8 & 2.4E-02 & 1678.3 & 361.6 & 111.0 & 119.1 & 133.7 & 5.5E-01 \\
\hline $3-223$ & 2.6 & 4.5 & 0.0 & 0.0 & 0.0 & $4.0 \mathrm{E}-02$ & 1.8 & 3.0 & 0.0 & 0.0 & 0.0 & 2.6E-02 \\
\hline 3-1404 & 1357.8 & 311.2 & 457.7 & 39.6 & 39.9 & $2.5 \mathrm{E}-01$ & 637.9 & 146.2 & 215.0 & 18.6 & 18.7 & $1.2 E-01$ \\
\hline $3-440$ & 41.2 & 8.9 & 2.7 & 2.9 & 3.3 & $1.3 \mathrm{E}-02$ & 27.8 & 6.0 & 1.8 & 2.0 & 2.2 & $9.0 \mathrm{E}-03$ \\
\hline $3-440$ & 109.4 & 25.1 & 36.9 & 3.2 & 3.2 & 2.0E-02 & 67.6 & 15.5 & 22.8 & 2.0 & 2.0 & 1.2E-02 \\
\hline $3-1076$ & 0.0 & 0.0 & 0.0 & 0.0 & 0.0 & $0.0 \mathrm{E}+00$ & 64.7 & 14.0 & 4.3 & 4.6 & 5.2 & 2.1E-02 \\
\hline $3-1498$ & 115.9 & 26.6 & 39.1 & 3.4 & 3.4 & 2.1E-02 & 49.7 & 11.4 & 16.7 & 1.4 & 1.5 & $9.0 \mathrm{E}-03$ \\
\hline 3-2322 & 67.2 & 14.5 & 4.4 & 4.8 & 5.4 & $2.2 \mathrm{E}-02$ & 104.3 & 22.5 & 6.9 & 7.4 & 8.3 & $3.4 \mathrm{E}-02$ \\
\hline $16-205$ & 215.1 & 46.4 & 14.2 & 15.3 & 17.1 & 7.0E-02 & 261.9 & 56.4 & 17.3 & 18.6 & 20.9 & 8.5E-02 \\
\hline $16-980$ & 0.0 & 0.0 & 0.0 & 0.0 & 0.0 & $0.0 E+00$ & 368.2 & 84.4 & 124.1 & 10.7 & 10.8 & 6.7E-02 \\
\hline $16-1374$ & 19.5 & 32.8 & 0.0 & 0.1 & 0.3 & $2.9 \mathrm{E}-01$ & 19.4 & 32.6 & 0.0 & 0.1 & 0.3 & 2.9E-01 \\
\hline $18-31$ & 68.6 & 14.8 & 4.5 & 4.9 & 5.5 & $2.2 \mathrm{E}-02$ & 69.6 & 15.0 & 4.6 & 4.9 & 5.5 & 2.3E-02 \\
\hline 21-155 & 272.8 & 62.5 & 91.9 & 8.0 & 8.0 & 4.9E-02 & 62.1 & 14.2 & 20.9 & 1.8 & 1.8 & 1.1E-02 \\
\hline 21-257 & 0.0 & 0.0 & 0.0 & 0.0 & 0.0 & $0.0 E+00$ & 0.0 & 0.0 & 0.0 & 0.0 & 0.0 & $0.0 \mathrm{E}+00$ \\
\hline $21-357$ & 56.1 & 12.1 & 3.7 & 4.0 & 4.5 & $1.8 \mathrm{E}-02$ & 17.8 & 3.8 & 1.2 & 1.3 & 1.4 & $5.8 \mathrm{E}-03$ \\
\hline 21-1002 & 37.8 & 8.2 & 2.5 & 2.7 & 3.0 & $1.2 \mathrm{E}-02$ & 14.5 & 3.1 & 1.0 & 1.0 & 1.2 & 4.7E-03 \\
\hline 21-1002 & 219.7 & 47.3 & 14.5 & 15.6 & 17.5 & 7.1E-02 & 945.3 & 203.7 & 62.5 & 67.1 & 75.3 & $3.1 \mathrm{E}-01$ \\
\hline 21-1002 & 13.1 & 2.8 & 0.9 & 0.9 & 1.0 & 4.3E-03 & 303.6 & 65.4 & 20.1 & 21.5 & 24.2 & $9.9 \mathrm{E}-02$ \\
\hline $33-87$ & 548.6 & 118.2 & 36.3 & 38.9 & 43.7 & $1.8 \mathrm{E}-01$ & 18.9 & 4.1 & 1.3 & 1.3 & 1.5 & $6.2 E-03$ \\
\hline 33-151 & 0.0 & 0.0 & 0.0 & 0.0 & 0.0 & $0.0 \mathrm{E}+00$ & 0.0 & 0.0 & 0.0 & 0.0 & 0.0 & $0.0 \mathrm{E}+00$ \\
\hline $33-208$ & 0.0 & 0.0 & 0.0 & 0.0 & 0.0 & $0.0 \mathrm{E}+00$ & 0.0 & 0.0 & 0.0 & 0.0 & 0.0 & $0.0 \mathrm{E}+00$ \\
\hline 33-Point & 0.0 & 0.0 & 0.0 & 0.0 & 0.0 & $0.0 \mathrm{E}+00$ & 0.0 & 0.0 & 0.0 & 0.0 & 0.0 & $0.0 \mathrm{E}+00$ \\
\hline $35-2$ & 18.5 & 4.0 & 1.2 & 1.3 & 1.5 & $6.0 \mathrm{E}-03$ & 5.6 & 1.2 & 0.4 & 0.4 & 0.4 & $1.8 \mathrm{E}-03$ \\
\hline 43-1 & 16.4 & 3.5 & 1.1 & 1.2 & 1.3 & 5.3E-03 & 23.2 & 5.0 & 1.5 & 1.6 & 1.8 & $7.5 \mathrm{E}-03$ \\
\hline 43-1 & 184.6 & 39.8 & 12.2 & 13.1 & 14.7 & $6.0 \mathrm{E}-02$ & 98.9 & 21.3 & 6.5 & 7.0 & 7.9 & 3.2E-02 \\
\hline 46-335 & 310.5 & 66.9 & 20.5 & 22.0 & 24.7 & $1.0 \mathrm{E}-01$ & 702.3 & 151.3 & 46.4 & 49.8 & 56.0 & 2.3E-01 \\
\hline $48-45$ & 30.7 & 6.6 & 2.0 & 2.2 & 2.4 & 1.0E-02 & 29.0 & 6.2 & 1.9 & 2.1 & 2.3 & 9.4E-03 \\
\hline $50-37$ & 22.5 & 5.2 & 7.6 & 0.7 & 0.7 & 4.1E-03 & 75.9 & 17.4 & 25.6 & 2.2 & 2.2 & $1.4 \mathrm{E}-02$ \\
\hline $50-184$ & 21.5 & 36.1 & 0.0 & 0.1 & 0.3 & 3.2E-01 & 22.4 & 37.7 & 0.0 & 0.1 & 0.3 & 3.3E-01 \\
\hline $50-188$ & 293.7 & 67.3 & 99.0 & 8.6 & 8.6 & 5.3E-02 & 206.9 & 47.4 & 69.7 & 6.0 & 6.1 & 3.7E-02 \\
\hline $53-1$ & 20.2 & 34.0 & 0.0 & 0.1 & 0.3 & 3.0E-01 & 20.9 & 35.3 & 0.0 & 0.1 & 0.3 & 3.1E-01 \\
\hline $53-2$ & 13.3 & 2.9 & 0.9 & 0.9 & 1.1 & 4.3E-03 & 11.2 & 2.4 & 0.7 & 0.8 & 0.9 & 3.7E-03 \\
\hline 53-M & 0.0 & 0.0 & 0.0 & 0.0 & 0.0 & $0.0 \mathrm{E}+00$ & 0.0 & 0.0 & 0.0 & 0.0 & 0.0 & $0.0 \mathrm{E}+00$ \\
\hline
\end{tabular}


2004 Generator Emissions

\begin{tabular}{|c|c|c|c|c|c|c|c|c|c|c|c|c|c|}
\hline $\mathbf{5 3 - M}$ & 0.0 & 0.0 & 0.0 & 0.0 & 0.0 & $0.0 \mathrm{E}+00$ & 0.0 & 0.0 & 0.0 & 0.0 & 0.0 & $0.0 \mathrm{E}+00$ \\
\hline $\mathbf{5 4 - 4 1 2}$ & 179.6 & 41.2 & 60.5 & 5.2 & 5.3 & $3.2 \mathrm{E}-02$ & 260.7 & 59.7 & 87.9 & 7.6 & 7.7 & $4.7 \mathrm{E}-02$ \\
\hline $\mathbf{5 5 - 5}$ & 6.9 & 11.7 & 0.0 & 0.0 & 0.1 & $1.0 \mathrm{E}-01$ & 6.9 & 11.6 & 0.0 & 0.0 & 0.1 & $1.0 \mathrm{E}-01$ \\
\hline $\mathbf{5 5 - 8}$ & 194.6 & 44.6 & 65.6 & 5.7 & 5.7 & $3.5 \mathrm{E}-02$ & 291.3 & 66.8 & 98.2 & 8.5 & 8.6 & $5.3 \mathrm{E}-02$ \\
\hline $\mathbf{5 5 - 2 8}$ & 10.5 & 2.3 & 0.7 & 0.7 & 0.8 & $3.4 \mathrm{E}-03$ & 10.5 & 2.3 & 0.7 & 0.7 & 0.8 & $3.4 \mathrm{E}-03$ \\
\hline $\mathbf{5 5 - 4 7}$ & 37.4 & 8.1 & 2.5 & 2.7 & 3.0 & $1.2 \mathrm{E}-02$ & 139.7 & 30.1 & 9.2 & 9.9 & 11.1 & $4.5 \mathrm{E}-02$ \\
\hline $\mathbf{5 5 - 1 4 2}$ & 18.0 & 3.9 & 1.2 & 1.3 & 1.4 & $5.8 \mathrm{E}-03$ & 0.0 & 0.0 & 0.0 & 0.0 & 0.0 & $0.0 \mathrm{E}+00$ \\
\hline $\mathbf{5 9 - 1}$ & 26.6 & 5.7 & 1.8 & 1.9 & 2.1 & $8.6 \mathrm{E}-03$ & 19.2 & 4.1 & 1.3 & 1.4 & 1.5 & $6.3 \mathrm{E}-03$ \\
\hline $\mathbf{6 1 - 2 3}$ & 0.0 & 0.0 & 0.0 & 0.0 & 0.0 & $0.0 \mathrm{E}+00$ & $\mathbf{9 . 2}$ & 2.0 & 0.6 & 0.7 & 0.7 & $3.0 \mathrm{E}-03$ \\
\hline $\mathbf{6 4 - 1}$ & 62.4 & 13.4 & 4.1 & 4.4 & 5.0 & $2.0 \mathrm{E}-02$ & 26.7 & $\mathbf{5 . 8}$ & 1.8 & 1.9 & 2.1 & $8.7 \mathrm{E}-03$ \\
\hline $\mathbf{6 9 - 3 3}$ & 0.0 & 0.0 & 0.0 & 0.0 & 0.0 & $0.0 \mathrm{E}+00$ & 663.8 & $\mathbf{1 5 2 . 1}$ & 223.8 & 19.4 & 19.5 & $1.2 \mathrm{E}-01$ \\
\hline Ibs/6 months & 4685.4 & 1148.3 & 995.1 & 221.4 & 240.6 & 2.2 & 7337.8 & 1722.7 & 1207.8 & 403.8 & 444.9 & 3.0 \\
\hline Tons/6 months & $\mathbf{2 . 3}$ & $\mathbf{0 . 6}$ & $\mathbf{0 . 5}$ & $\mathbf{0 . 1}$ & $\mathbf{0 . 1}$ & $\mathbf{1 . 1 \mathrm { E } - 0 3}$ & $\mathbf{3 . 7}$ & $\mathbf{0 . 9}$ & $\mathbf{0 . 6}$ & $\mathbf{0 . 2}$ & $\mathbf{0 . 2}$ & $\mathbf{1 . 5 E}-\mathbf{0 3}$ \\
\hline
\end{tabular}

\begin{tabular}{|l|c|c|c|c|c|c|}
\hline YEARLY TOTAL & NOx & CO & SOX & PM & VOC & HAPs \\
\hline Tons/Year & 6.0 & 1.4 & 1.1 & 0.3 & 0.3 & 0.003 \\
\hline
\end{tabular}


ATTACHMENT B.

2004 ANNUAL EMISSIONS INVENTORY SUBMITTAL TO NMED 
Associate Directorate for Technical Services

P.O. Box 1663, A104

Los Alamos, New Mexico 87545

505-667-0079/Fax 505-665-1812
Date: March 22, 2005

Refer To: ADTS:05-022

Ms. Heather Lancour

New Mexico Environment Department

Air Quality Bureau

2048 Galisteo Street

Santa Fe, NM 87505

\section{IDEA ID NO.856 - LOS ALAMOS NATIONAL LABORATORY (LANL) ANNUAL EMISSION INVENTORY REPORT REQUIRED UNDER 20.2.73 NMAC}

Dear Ms. Lancour:

Enclosed is the 2004 Emissions Inventory Update for Los Alamos National Laboratory (LANL or Laboratory), required by Title 20, Chapter 2, Part 73 of the New Mexico Administrative Code (20.2.73 NMAC), Notice of Intent and Emissions Inventory Requirements.

We have updated the eight Excel worksheets that you provided, using the guidance in your instructions for the 2004 New Mexico Emissions Inventory Update. All changes made to the worksheets are highlighted in yellow (additions) and green (deletions).

In general, criteria pollutant emissions from LANL sources in 2004 are slightly lower than reported in previous years. Additionally, please note the following changes to our inventory for 2004:

- The following emission units were removed from operation during 2004: beryllium-machining operation (AIRS \#008) at TA-3, Building 102; Plant 6 boiler and backup (AIRS \#025) at TA-16, Building 1485; rock crusher (AIRS \#026); and paper shredder (AIRS \#027).

- One emission unit is reported for the first time this year: a data disintegrator (AIRS \#045) located at TA-52. This unit replaced the paper shedder that was removed in July, 2004.

For the first time, NMED has required the reporting of hazardous air pollutant (HAP) emissions, particulate matter in the size of 2.5 microns in diameter or less $\left(\mathbf{P M}_{2.5}\right)$ emissions, and ammonia $\left(\mathrm{NH}_{3}\right)$ emissions in the 2004 Emissions Inventory. As requested by NMED, LANL has voluntarily reported these emissions in the past. Therefore, this information, with the exception of the radionuclide emissions, is once again included in the 2004 Emissions Inventory.

Emissions of radionuclides other than radon from Laboratory operations, as reported to EPA under 40 CFR 61 Subpart $\mathrm{H}$, resulted in a maximum offsite dose of $0.65 \mathrm{mrem}$ during 2003. For 2004, this offsite dose is estimated to be approximately $2 \mathrm{mrem}$. A final dose for 2004 will be reported to EPA in June 2005.

This submittal includes print outs of the eight updated spreadsheets for LANL, as well as a diskette containing electronic copies of the updated spreadsheets. We have also included a summary report that was prepared at the completion of the Emissions Inventory submittal for 2003. We followed the same methodology in preparing the 2004 emissions inventory updates as described in this report. 
If you have any questions regarding this report, please contact Margie Stockton (667-9359) or Walt Whetham (6658885), in the Laboratory's Meteorology and Air Quality Group.

Sincerely,

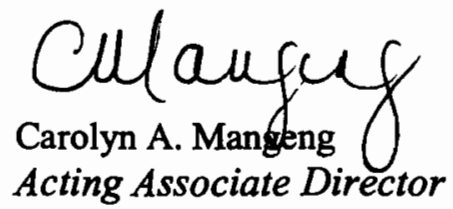

CAM:alb

Cy:

S. Fong, DOE/OLASO, A316

K. Hargis, ENV-DO, J591

D. Stavert, ENV-DO, J591

P. Wardwell, LC-ESH, A187

J. Hurtle, ENV-MAQ, J978

D. Wilburn, ENV -MAQ, J978

S. Story, ENV -MAQ, J978

J. Dewart, ENV-MAQ, J978

W. Whetham, ENV -MAQ, J978

M. Stockton, ENV-MAQ, J978

20.2.73 NMAC Project File

ENV-MAQ File 


\title{
LOS ALAMOS NATIONAL LABORATORY'S 2004 EMISSIONS INVENTORY
}

Submitted as Required by:

Title 20, Chapter 2, Part 73 of the New Mexico Administrative Code

Prepared by:

\author{
The University of California
}

For:

\section{The National Nuclear Security Administration of the United States Department of Energy}

Information Contacts:

Margie Stockton, (505) 667-9359

Jean Dewart, (505) 665-0239

\section{Certification Statement}

I, Carolyn A. Mangeng, hereby certify on behalf of Los Alamos National Laboratory and the University of California, that the information and statements contained in this Emissions Inventory report are true and accurate to the best of my knowledge and belief.
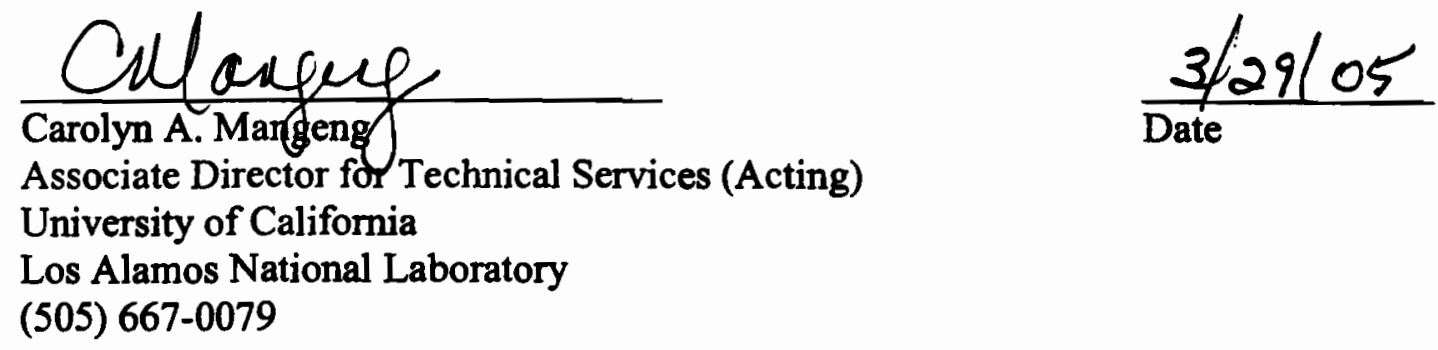


\begin{tabular}{|c|c|c|c|c|c|c|c|c|c|c|c|c|c|c|}
\hline County & $\begin{array}{l}\text { County } \\
\text { FIPS }\end{array}$ & $\begin{array}{c}\text { AFS/NEDS } \\
\text { Plant ID }\end{array}$ & $\begin{array}{c}\text { Inventory } \\
\text { Year } \\
\text { (mmy }\end{array}$ & \begin{tabular}{|c|} 
Air \\
Program \\
Status \\
\end{tabular} & SIC & NAICS & Company Name & Facility Name & Facility Description & Street Line 1 & Street Name 2 & City & State & Zip Code \\
\hline Los Alamos & 028 & 0001 & 2004 & & 9711 & & $\begin{array}{l}\text { Los Alamos National } \\
\text { Laboratory }\end{array}$ & $\begin{array}{l}\text { Los Alamos National } \\
\text { Laboratory }\end{array}$ & National Security & $\begin{array}{l}\text { PO Box } 1663 \text {, Air } \\
\text { Quality Gro }\end{array}$ & Mail Stop J978 & $\begin{array}{l}\text { Los } \\
\text { Alamos }\end{array}$ & NM & 87545- \\
\hline
\end{tabular}




\begin{tabular}{|c|c|c|c|c|c|c|c|c|c|c|c|c|}
\hline $\begin{array}{c}\text { Contact } \\
\text { Person Name }\end{array}$ & $\begin{array}{c}\text { Contact Person } \\
\text { Phone }\end{array}$ & $\begin{array}{c}\text { Contact Person E- } \\
\text { mail }\end{array}$ & $\begin{array}{l}\text { UTM } \\
\text { Zone } \\
\end{array}$ & UTMH & UTMV & \begin{tabular}{|c|}
$\begin{array}{c}\text { Elevation } \\
\text { (ft) }\end{array}$ \\
\end{tabular} & Street Line 1 & City & Permit Number & Permit Type & strClass & $\begin{array}{c}\text { Facility } \\
\text { Category Code }\end{array}$ \\
\hline Jean Dewart & 5056650239 & dewart@lanl.gov & 13 & 381.19 & 3970.3 & 7380 & $\begin{array}{l}1 \mathrm{mi} \mathrm{S} \text { of Los } \\
\text { Alamos }\end{array}$ & $\begin{array}{l}\text { Los } \\
\text { Alamos }\end{array}$ & $\begin{array}{l}\text { 1081M1R3,2195,2195B,0632,0634M2,0635,0636, } \\
\text { 0632, 0634, 1081, 2195, 2195B, 2195F, 2195H,GCP-3- } \\
\text { 2195G, P100 }\end{array}$ & $\begin{array}{l}\text {-Regular } \\
\text { Permit }\end{array}$ & A & \\
\hline
\end{tabular}




\begin{tabular}{|c|c|c|c|c|c|c|c|c|c|}
\hline Eounty FIPS & $\begin{array}{c}\text { AFS/NEDS Plant } \\
\text { ID }\end{array}$ & \begin{tabular}{|c|} 
Inventory \\
Year \\
(ymy
\end{tabular} & Facility Name & $\begin{array}{c}\text { Emission } \\
\text { Type }\end{array}$ & Pollutant Code & CAS Number & \begin{tabular}{|c|c|} 
Emission Numeric \\
Value
\end{tabular} & $\begin{array}{c}\text { Emission Unit } \\
\text { Numerator }\end{array}$ & strClass \\
\hline 028 & 0001 & 2004 & Los Alamos National & 12 & 100027 & 100027 & $2.50 \mathrm{E}-05 \mathrm{~T}$ & TY & $\mathrm{B}$ \\
\hline 028 & 0001 & 2004 & Los Alamos National & 12 & 100414 & 100414 & & TY & B \\
\hline 028 & 0001 & 2004 & Los Alamos National & 12 & 100425 & 100425 & 2.25E-03 T & TY & B \\
\hline 028 & 0001 & 2004 & Los Alamos National & 12 & 100447 & 100447 & 1.20E-04 T & TY & B \\
\hline 028 & 0001 & 2004 & Los Alamos National & 12 & 101688 & 101688 & $0 T$ & TY & B \\
\hline 028 & 0001 & 2004 & Los Alamos National & 12 & 106423 & 106423 & $2.08 \mathrm{E}-03 \mathrm{~T}$ & TY & 8 \\
\hline 028 & 0001 & 2004 & Los Alamos National & 12 & 106445 & & 1.10E-04 T & TY & B \\
\hline 028 & 0001 & 2004 & Los Alamos National & 12 & 106467 & 106467 & 4.36E-04 T & TY & B \\
\hline 028 & 0001 & 2004 & Los Alamos National & 12 & 106503 & 106503 & $5.50 \mathrm{E}-04 \mathrm{~T}$ & TY & B \\
\hline 028 & 0001 & 2004 & Los Alamos National & 12 & 106514 & 106514 & OT & TY & B \\
\hline 028 & 0001 & 2004 & Los Alamos National & 12 & 106887 & 106887 & 1.75E-04 T & TY & B \\
\hline 028 & 0001 & 2004 & Los Alamos National & 12 & 106898 & 106898 & $0 T$ & TY & B \\
\hline 028 & 0001 & 2004 & Los Alamos National & 12 & 106934 & 106934 & 1.10E-04 T & TY & B \\
\hline 028 & 0001 & 2004 & Los Alamos National & 12 & 106990 & 106990 & $0 T$ & TY & B \\
\hline 028 & 0001 & 2004 & Los Alamos National & 12 & 107028 & 107028 & $0 T$ & TY & B \\
\hline 028 & 0001 & 2004 & Los Alamos National & 12 & 107062 & 107062 & 4.77E-03 T & TY & B \\
\hline 028 & 0001 & 2004 & Los Alamos National & 12 & 107131 & 107131 & OT & TY & B \\
\hline 028 & 0001 & 2004 & Los Alamos National & 12 & 107211 & 107211 & 2.56E-01 T & TY & B \\
\hline 028 & 0001 & 2004 & Los Alamos National & 12 & 107302 & 107302 & & TY & B \\
\hline 028 & 0001 & 2004 & Los Alamos National & 12 & 108054 & 108054 & & TY & B \\
\hline 028 & 0001 & 2004 & Los Alamos National & 12 & 108101 & 108101 & & TY & B \\
\hline 028 & 0001 & 2004 & Los Alamos National & 12 & 108383 & 108383 & $2.58 \mathrm{E}-03 \mathrm{~T}$ & TY & B \\
\hline 028 & 0001 & 2004 & Los Alamos National & 12 & 108394 & 108394 & 1.10E-04 T & TY & $B$ \\
\hline 028 & 0001 & 2004 & Los Alamos National & 12 & 108394 & 108394 & 1.10E-04 T & TY & B \\
\hline 028 & 0001 & 2004 & Los Alamos National & 12 & 108907 & 108907 & $1.21 \mathrm{E}-02 \mathrm{~T}$ & TY & B \\
\hline 028 & 0001 & 2004 & Los Alamos National & 12 & 108952 & 108952 & 1.91E-03 T & TY & B \\
\hline 028 & 0001 & 2004 & Los Alamos National & 12 & 109 & 109 & 3.26E-04 T & TY & B \\
\hline 028 & 0001 & 2004 & Los Alamos National & 12 & 110543 & 110543 & $8.84 \mathrm{E}-01 \mathrm{~T}$ & TY & B \\
\hline 028 & 0001 & 2004 & Los Alamos National & 12 & 111422 & 111422 & $6.00 \mathrm{E}-04 \mathrm{~T}$ & TY & B \\
\hline 028 & 0001 & 2004 & Los Alamos National & 12 & 1120714 & 1120714 & & TY & B \\
\hline 028 & 0001 & 2004 & Los Alamos National & 12 & 117817 & 117817 & 5.00E-06 T & TY & B \\
\hline 028 & 0001 & 2004 & Los Alamos National & 12 & 120809 & 120809 & $5.50 \mathrm{E}-04 \mathrm{~T}$ & TY & B \\
\hline 028 & 0001 & 2004 & Los Alamos National & 12 & 120821 & 120821 & & TY & B \\
\hline 028 & 0001 & 2004 & Los Alamos National & 12 & 121448 & 121448 & 5.37E-03 T & TY & B \\
\hline 028 & 0001 & 2004 & Los Alamos National & 12 & 121697 & 121697 & & TY & $\mathrm{B}$ \\
\hline 028 & 0001 & 2004 & Los Alamos National & 12 & 122667 & & & TY & B \\
\hline 028 & 0001 & 2004 & Los Alamos National & 12 & 123319 & 123319 & 1.05E-04 T & TY & B \\
\hline 028 & 0001 & 2004 & Los Alamos National & 12 & 123386 & 123386 & & TY & B \\
\hline 028 & 0001 & 2004 & Los Alamos National & 12 & 123911 & 123911 & 3.43E-03 T & TY & B \\
\hline 028 & 0001 & 2004 & Los Alamos National & 12 & 125 & 125 & 1.05E-03 T & TY & B \\
\hline 028 & 0001 & 2004 & Los Alamos National & 12 & 127184 & 127184 & & TY & $B$ \\
\hline 028 & 0001 & 2004 & Los Alamos National & 12 & 136 & 136 & 4.24E-03 T & TY & B \\
\hline 028 & 0001 & 2004 & Los Alamos National & 12 & 139 & 139 & 2.69E-03 T & TY & B \\
\hline 028 & 0001 & 2004 & Los Alamos National & 12 & 140885 & 140885 & & TY & B \\
\hline 028 & 0001 & 2004 & Los Alamos National & 12 & 144 & 144 & 5.65E-02 T & TY & B \\
\hline 028 & 0001 & 2004 & Los Alamos National & 12 & 156627 & & & TY & B \\
\hline 028 & 0001 & 2004 & Los Alamos National & 12 & 1634044 & 1634044 & $1.03 \mathrm{E}-02 \mathrm{~T}$ & TY & B \\
\hline 028 & 0001 & 2004 & Los Alamos National & 12 & 171 & 171 & $4.50 \mathrm{E}-02 \mathrm{~T}$ & TY & B \\
\hline 028 & 0001 & 2004 & Los Alamos National & 12 & 1746016 & 1746016 & & TY & B \\
\hline 028 & 0001 & 2004 & Los Alamos National & 12 & 18540299 & 18540299 & & TY & B \\
\hline 028 & 0001 & 2004 & Los Alamos National & 12 & 195 & 195 & 1.83E-03 T & TY & B \\
\hline 028 & 0001 & 2004 & Los Alamos National & 12 & 198 & 198 & $5.15 \mathrm{E}-01 \mathrm{~T}$ & TY & B \\
\hline 028 & 0001 & 2004 & Los Alamos National & 12 & 199 & 199 & 1.07E-03 T & TY & B \\
\hline 028 & 0001 & 2004 & Los Alamos National & 12 & 226 & 226 & $6.14 \mathrm{E}-03 \mathrm{~T}$ & TY & B \\
\hline 028 & 0001 & 2004 & Los Alamos National & 12 & 234 & 234 & $0 \mathrm{~T}$ & TY & B \\
\hline 028 & 0001 & 2004 & Los Alamos National & 12 & 246 & 246 & 4.89E-03 T & TY & B \\
\hline 028 & 0001 & 2004 & Los Alamos National & 12 & 253 & 253 & 1.01E-02 T & TY & B \\
\hline 028 & 0001 & 2004 & Los Alamos National & 12 & 302012 & 302012 & 1.10E-03 T & TY & B \\
\hline 028 & 0001 & 2004 & Los Alamos National & 12 & 3CLET & 79016 & 1.10E-02 T & TY & B \\
\hline 028 & 0001 & 2004 & Los Alamos National & 12 & 463581 & 463581 & $0 T$ & TY & B \\
\hline 028 & 0001 & 2004 & Los Alamos National & 12 & 51207319 & 51207319 & $0 T$ & TY & B \\
\hline 028 & 0001 & 2004 & Los Alamos National & 12 & 51285 & 51285 & & TY & B \\
\hline 028 & 0001 & 2004 & Los Alamos National & 12 & 51796 & 51796 & 7.35E-04 T & TY & B \\
\hline 028 & 0001 & $2004 \mid$ & Los Alamos National & 12 & 540841 & 540841 & $4.58 \mathrm{E}-0.3 \mathrm{~T}$ & TY & B \\
\hline 028 & 0001 & 2004 & Los Alamos National & 12 & 542884 & 542881 & $0 T$ & TY & B \\
\hline 028 & 0001 & 2004 & Los Alamos National & 12 & 56235 & 56235 & 8.79E-03 T & TY & B \\
\hline 028 & 0001 & 2004 & Los Alamos National & 12 & 57125 & 57125 & $0 T$ & TY & B \\
\hline 028 & 0001 & 2004 & Los Alamos National & 12 & 57147 & 57147 & $0 \mathrm{~T}$ & TY & $B$ \\
\hline 028 & 0001 & 2004 & Los Alamos National & $\sqrt{12}$ & 584849 & 584849 & $0 \mathrm{~T}$ & TY & B \\
\hline 028 & 0001 & 2004 & Los Alamos National & 12 & 60344 & & $0 T$ & TY & B \\
\hline 028 & 0001 & 2004 & Los Alamos National & 12 & 624 & 624 & OT & TY & B \\
\hline 028 & 0001 & 2004 & Los Alamos National & 12 & 62533 & 62533 & $6.45 \mathrm{E}-0.4 \mathrm{~T}$ & TY & B \\
\hline 028 & 0001 & 2004 & Los Alamos National & 12 & 67561 & 67561 & 7.29E-01 T & TY & B \\
\hline 028 & 0001 & 2004 & Los Alamos National & 12 & 67663 & 67663 & 1.25E-01 T & TY & B \\
\hline 028 & 0001 & 2004 & Los Alamos National & 12 & 680319 & 680319 & $0 T$ & TY & B \\
\hline 028 & 0001 & 2004 & Los Alamos National & 12 & 68122 & 68122 & $1.40 E-01 T$ & TY & B \\
\hline
\end{tabular}




\begin{tabular}{|c|c|c|c|c|c|c|c|}
\hline 028 & 0001 & 2004 Los Alamos National & 12 & 71556 & 71556 & OTY & B \\
\hline 028 & 0001 & 2004 Los Alamos National & 12 & 7439921 & 7439921 & 0 TY & B \\
\hline 028 & 0001 & 2004 Los Alamos National & 12 & 7439976 & 7439976 & 0 TY & B \\
\hline 028 & 0001 & 2004 Los Alamos National & 12 & 7440020 & 7440020 & $0 T Y$ & B \\
\hline 028 & 0001 & 2004 Los Alamos National & 12 & 7440360 & 7440360 & 0 TY & B \\
\hline 028 & 0001 & 2004 Los Alamos National & 12 & 7440382 & 7440382 & OTY & B \\
\hline 028 & 0001 & 2004 Los Alamos National & 12 & 7440417 & 7440417 & 0 TY & B \\
\hline 028 & 0001 & 2004 Los Alamos National & 12 & 7440439 & 7440439 & $0 T Y$ & B \\
\hline 028 & 0001 & 2004 Los Alamos National & 12 & 7440473 & 7440473 & $0 \mathrm{TY}$ & B \\
\hline 028 & 0001 & 2004 Los Alamos National & 12 & 7440484 & 7440484 & OTY & B \\
\hline 028 & 0001 & 2004 Los Alamos National & 12 & 74839 & 74839 & 0 TY & B \\
\hline 028 & 0001 & 2004 Los Alamos National & 12 & 74873 & 74873 & 0 TY & B \\
\hline 028 & 0001 & 2004 Los Alamos National & 12 & 74884 & 74884 & 0 TY & B \\
\hline 028 & 0001 & 2004 Los Alamos National & 12 & 75003 & 75003 & 0 TY & B \\
\hline 028 & 0001 & 2004 Los Alamos National & 12 & 75014 & 75014 & OTY & B \\
\hline 028 & 0001 & 2004 Los Alamos National & 12 & 75058 & 75058 & 7.03E-01 TY & B \\
\hline 028 & 0001 & 2004 Los Alamos National & 12 & 75070 & 75070 & OTY & B \\
\hline 028 & 0001 & 2004 Los Alamos National & 12 & 75092 & 75092 & 6.32E-01 TY & B \\
\hline 028 & 0001 & 2004 Los Alamos National & 12 & 75150 & 75150 & 3.00E-05 TY & B \\
\hline 028 & 0001 & 2004 Los Alamos National & 12 & 75252 & & 7.50E-05 TY & B \\
\hline 028 & 0001 & 2004 Los Alamos National & 12 & 75343 & 75343 & 0 TY & B \\
\hline 028 & 0001 & 2004 Los Alamos National & 12 & 75354 & 75354 & $0 T Y$ & B \\
\hline 028 & 0001 & 2004 Los Alamos National & 12 & 7550450 & 7550450 & 4.75E-04 TY & B \\
\hline 028 & 0001 & 2004 Los Alamos National & 12 & 75569 & 75569 & 3.50E-05 TY & B \\
\hline 028 & 0001 & 2004 Los Alamos National & 12 & 7664393 & 7664393 & 2.09E-02 TY & B \\
\hline 028 & 0001 & 2004 Los Alamos National & 12 & 7723140 & 7723140 & 5.00E-04 TY & B \\
\hline 028 & 0001 & 2004 Los Alamos National & 12 & 77474 & 77474 & OTY & B \\
\hline 028 & 0001 & 2004 Los Alamos National & 12 & 77781 & 77781 & 1.00E-05 TY & B \\
\hline 028 & 0001 & 2004 Los Alamos National & 12 & 7782492 & 7782492 & OTY & B \\
\hline 028 & 0001 & 2004 Los Alamos National & 12 & 7803512 & 7803512 & OTY & B \\
\hline 028 & 0001 & 2004 Los Alamos National & 12 & 78875 & 78875 & OTY & B \\
\hline 028 & 0001 & 2004 Los Alamos National & 12 & 78933 & & 0 TY & B \\
\hline 028 & 0001 & 2004 Los Alamos National & 12 & 79005 & 79005 & 0 TY & B \\
\hline 028 & 0001 & 2004 Los Alamos National & 12 & 79016 & & OTY & B \\
\hline 028 & 0001 & 2004 Los Alamos National & 12 & 79061 & 79061 & 5.45E-03 TY & B \\
\hline 028 & 0001 & 2004 Los Alamos National & 12 & 79107 & 79107 & OTY & B \\
\hline 028 & 0001 & 2004 Los Alamos National & 12 & 79118 & 79118 & $0 T Y$ & B \\
\hline 028 & 0001 & 2004 Los Alamos National & 12 & 79345 & 79345 & $0 T Y$ & B \\
\hline 028 & 0001 & 2004 Los Alamos National & 12 & 80626 & 80626 & 3.17E-03 TY & B \\
\hline 028 & 0001 & 2004 Los Alamos National & 12 & 822060 & & 2.50E-05 TY & B \\
\hline 028 & 0001 & 2004 Los Alamos National & 12 & 84742 & 84742 & 3.31E-03 TY & B \\
\hline 028 & 0001 & 2004 Los Alamos National & 12 & 85449 & 85449 & OTY & B \\
\hline 028 & 0001 & 2004 Los Alamos National & 12 & 87865 & 87865 & OTY & B \\
\hline 028 & 0001 & 2004 Los Alamos National & 12 & 88062 & 88062 & OTY & B \\
\hline 028 & 0001 & 2004 Los Alamos National & 12 & 91203 & 91203 & 5.02E-04 TY & B \\
\hline 028 & 0001 & 2004 Los Alamos National & 12 & 91225 & 91225 & 1.15E-04 TY & B \\
\hline 028 & 0001 & 2004 Los Alamos National & 12 & 92 & 92 & 6.60E-04 TY & B \\
\hline 028 & 0001 & 2004 Los Alamos National & 12 & 92524 & 92524 & OTY & B \\
\hline 028 & 0001 & 2004 Los Alamos National & 12 & 92671 & 92671 & OTY & B \\
\hline 028 & 0001 & 2004 Los Alamos National & 12 & 93 & 93 & 1.58E-03 TY & B \\
\hline 028 & 0001 & 2004 Los Alamos National & 12 & 95476 & 95476 & 5.82E-03 TY & B \\
\hline 028 & 0001 & 2004 Los Alamos National & 12 & 98828 & & OTY & B \\
\hline 028 & 0001 & 2004 Los Alamos National & 12 & 98862 & 98862 & $5.60 \mathrm{E}-04 \mathrm{TY}$ & B \\
\hline 028 & 0001 & 2004 Los Alamos National & 12 & 98953 & 98953 & 1.32E-03 TY & B \\
\hline 028 & 0001 & 2004 Los Alamos National & 12 & $A B$ & & OTY & B \\
\hline 028 & 0001 & 2004 Los Alamos National & 12 & AL-PT & 7429905 & 3.30E-06 TY & B \\
\hline 028 & 0001 & 2004 Los Alamos National & 12 & $\mathrm{BE}$ & 7440417 & 3.70E-06 TY & B \\
\hline 028 & 0001 & 2004 Los Alamos National & 12 & $B Z$ & 71432 & 1.19E-02 TY & B \\
\hline 028 & 0001 & 2004 Los Alamos National & 12 & $\mathrm{CL}$ & 7782505 & 1.28E-02 TY & B \\
\hline 028 & 0001 & 2004 Los Alamos National & 12 & $\mathrm{CO}$ & 630080 & 1.71E+01 TY & A \\
\hline 028 & 0001 & 2004 Los Alamos National & 12 & FORM & 50000 & 9.38E-02 TY & B \\
\hline 028 & 0001 & 2004 Los Alamos National & 12 & $\mathrm{HC} 36$ & 78933 & 3.64E-01 TY & B \\
\hline 028 & 0001 & 2004 Los Alamos National & 12 & $\mathrm{HC} 81$ & 1330207 & 4.16E-03 TY & B \\
\hline 028 & 0001 & 2004 Los Alamos National & 12 & $\mathrm{HCL}$ & 7647010 & $1.52 \mathrm{E}+00 \mathrm{TY}$ & B \\
\hline 028 & 0001 & 2004 Los Alamos National & 12 & MN-PT & 7439965 & OTY & B \\
\hline 028 & 0001 & 2004 Los Alamos National & 12 & $\mathrm{NH} 3$ & 7664417 & 5.91E-02 TY & B \\
\hline 028 & 0001 & 2004 Los Alamos National & 12 & $\mathrm{NO} 2$ & 10102440 & $2.45 E+01 T Y$ & A \\
\hline 028 & 0001 & 2004 Los Alamos National & 12 & PM10 & & $2.95 \mathrm{E}+00 \mathrm{TY}$ & B \\
\hline 028 & 0001 & 2004 Los Alamos National & 12 & PM25 & & $2.93 \mathrm{E}+00 \mathrm{TY}$ & B \\
\hline 028 & 0001 & 2004 Los Alamos National & 12 & PT & & $3.03 E+00 T Y$ & B \\
\hline 028 & 0001 & 2004 Los Alamos National & 12 & $\mathrm{RD}$ & & OTY & B \\
\hline 028 & 0001 & 2004 Los Alamos National & 12 & $\mathrm{SO} 2$ & 7446095 & 3.43E-01 TY & B \\
\hline 028 & 0001 & 2004 Los Alamos National & 12 & THAP & & $6.38 \mathrm{E}+00 \mathrm{TY}$ & B \\
\hline 028 & 0001 & 2004 Los Alamos National & 12 & TOLU & 108883 & 1.43E-01 TY & B \\
\hline 028 & 0001 & 2004 Los Alamos National & 12 & VOC & & $1.00 \mathrm{E}+01 \mathrm{TY}$ & A \\
\hline
\end{tabular}




\begin{tabular}{|c|c|c|c|c|c|c|c|c|c|}
\hline Facility Name & $\begin{array}{c}\text { AIRS } \\
\text { Point ID } \\
\end{array}$ & $\begin{array}{c}\text { Design } \\
\text { Capacity }\end{array}$ & \begin{tabular}{|c|} 
Design \\
Capacity Unit \\
Numerator \\
\end{tabular} & $\begin{array}{c}\text { Design } \\
\text { Capacity } \\
\text { Unit }\end{array}$ & $\begin{array}{c}\text { Max } \\
\text { Nameplate } \\
\text { Capacity }\end{array}$ & \begin{tabular}{|c} 
Description \\
\end{tabular} & Installation Date $\mathbf{R}$ & Removal Date|: & Shut Down? \\
\hline Los Alamos National & 001 & & LB & YR & & Be Machining TA-3 BLDG 39 & 01-Jan-85 & 22-Oct-02 & TRUE \\
\hline Los Alamos National & 002 & & E6BTU & $\mathrm{HR}$ & & TA3-22 Boilers (3 Each) See Pt 32, 33, 34 & 01-Jan-50 & $27-$ Sep-00 & TRUE \\
\hline Los Alamos National & 003 & & E6BTU & HR & & TA16 Boiler (3 Each) - Removed & $01-J a n-50$ & 01-Jan-97 & TRUE \\
\hline Los Alamos National & 004 & 30.6 & E6BTU & HR & & Steam Plant Boiler TA21 BLDG 357 (3) & 01-Jan-83 & & FALSE \\
\hline Los Alamos National & 005 & & E6BTU & HR & & TD Site Boiler (Not Built) & & & FALSE \\
\hline Los Alamos National & 006 & 10000 & & YR & 10000 & Be Machining TA35 BLDG 213 & 26-Dec-85 & & FALSE \\
\hline Los Alamos National & 007 & 10000 & LB & YR & 10000 & Be Machining TA3 BLDG 141 & 19-Mar-85 & & FALSE \\
\hline Los Alamos National & 008 & & LB & YR & & Be Machining TA3 BLDG 102 & 19-Mar-86 & $20-F e b-04$ & TRUE \\
\hline Los Alamos National & 009 & & LB & YR & & Be Shop TA3-35 (Not Built) & & $22-0 \mathrm{ct}-02$ & TRUE \\
\hline Los Alamos National & 010 & 1100 & LB & YR & 1100 & Be Cutting \& Beand Dressing TA-55-4 & 01-Jan-78 & & FALSE \\
\hline Los Alamos National & 011 & 1100 & LB & YR & 1100 & Metallography TA55-4 North Stack & 01-Jan-78 & & FALSE \\
\hline Los Alamos National & 012 & & E6BTU & HR & & Solid Waste Fired Boiler (Not Built) & & & FALSE \\
\hline Los Alamos National & 013 & & TON & HR & & Asphalt Plant TA-3-73 & $01-J a n-60$ & 01-Jul-03 & TRUE \\
\hline Los Alamos National & 014 & & HP & HR & 700 & Caterpillar HCR TA SI Pump Engine & 01-Jan-82 & 01-Nov-01 & TRUE \\
\hline Los Alamos National & 015 & & E6BTU & HR & 6.3 & Boiler (TA-48-1) BS-1 & 01-Aug-87 & & FALSE \\
\hline Los Alamos National & 016 & & E6BTU & HR & 6.3 & Boiler (TA-48-1) BS-2 & $01-0 c t-76$ & & FALSE \\
\hline Los Alamos National & 017 & & E6BTU & HR & & Boiler (TA-48-1) BS-6 & $01-$ Oct-94 & & FALSE \\
\hline Los Alamos National & 018 & & E6BTU & HR & & Boiler (TA-53-365) BHW-1 & 01-Jan-88 & & FALSE \\
\hline Los Alamos National & 019 & & E6BTU & HR & & Boiler (TA-53-365) BHW-2 & 01-Jan-88 & & FALSE \\
\hline Los Alamos National & 020 & & E6BTU & HR & 6.3 & Boiler (TA-59-1) BHW-1 & $01-S e p-78$ & & FALSE \\
\hline Los Alamos National & 021 & & E6BTU & HR & 6.3 & Boiler (TA-59-1) BHW-2 & $01-$ Oct-94 & & FALSE \\
\hline Los Alamos National & 022 & & E6BTU & HR & 8.4 & Boiler (TA-55-6) BHW-1 & 01-Jan-76 & 01-Oct-98 & TRUE \\
\hline Los Alamos National & 023 & & E6BTU & HR & 8.4 & Boiler (TA-55-6) BHW-2 & 01-Jan-76 & $01-0 c t-01$ & TRUE \\
\hline Los Alamos National & 024 & 12.7 & E6BTU & HR & & Boiler and backup, TA-16, Plant- 5 & 01-Nov-96 & & FALSE \\
\hline Los Alamos National & 025 & 15.6 & E6BTU & HR & 18.4 & Boiler and Backup, Plant 6 (TA-16-1485) & 01-Nov-96 & 01-Jan-04 & TRUE \\
\hline Los Alamos National & 026 & & TON & HR & 150 & Rock Crusher & 01-Jun-98 & 10-Jun-04 & TRUE \\
\hline Los Alamos National & 027 & 300 & LB & HR & 300 & SEM-1424 Disintegrator paper shredder (TA- & 01-Jan-91 & 06-Jul-04 & TRUE \\
\hline Los Alamos National & 028 & 20 & & DAY & & Degreaser - cold ultrasonic bath TA-55-4 & 01-Sep-98 & & FALSE \\
\hline Los Alamos National & 029 & 18 & & DAY & & Degreaser - cold ultrasonic bath TA-46-24 & 03-Jun-00 & 06-Dec-01 & TRUE \\
\hline Los Alamos National & 030 & & L & DAY & & Degreaser - inhouse cold batch TA-55-4 & 01-Dec-99 & 29-Jan-01 & TRUE \\
\hline Los Alamos National & 031 & & LB & YR & & Research \& Development Activities - & 01-Jan-50 & & FALSE \\
\hline Los Alamos National & 032 & 189.5 & E6BTU & HR & 210 & TA3-22 Edgemoor Iron Works Boiler w/FGR & $01-J a n-50$ & & FALSE \\
\hline Los Alamos National & 033 & 189.5 & E6BTU & HR & 210 & TA3-22 Edgemoor Iron Works Boiler w/FGR & 01-Jan-50 & & FALSE \\
\hline Los Alamos National & 034 & 189.5 & E6BTU & HR & 210 & TA3-22 Edgemoor Iron Works Boiler w/FGR & $01-J a n-50$ & & FALSE \\
\hline Los Alamos National & 035 & 3770 & BBL & DAY & 3770 & Tank TA-03-026 (No. 2 fuel oil) & 01-Jan-52 & & FALSE \\
\hline Los Alamos National & 036 & 5455 & BBL & DAY & 5455 & Tank TA-03-779 (No. 2 fuel oil) & 01-Aug-98 & & FALSE \\
\hline Los Alamos National & 037 & 12.4 & E6BTU & HR & 14.6 & Sellers Boiler BHW-1B(TA 55, Bldg. PF6) & $01-$ Oct-98 & & FALSE \\
\hline Los Alamos National & 038 & 12.4 & E6BTU & HR & 14.6 & Sellers Boiler BHW-2B(TA 55, Bldg. PF6) & 06-Sep-01 & & FALSE \\
\hline Los Alamos National & 039 & & TON & HR & & Air Curtain Destructor S-127 & 29-Oct-01 & 01-Oct-03 & TRUE \\
\hline Los Alamos National & 040 & & TON & HR & & Air Curtain Destructor T-350-1 & 26-Sep-01 & 01-Oct-03 & TRUE \\
\hline Los Alamos National & 041 & & TON & HR & & Air Curtain Destructor T-350-2 & $11-$ Oct-01 & $01-0 c t-03$ & TRUE \\
\hline Los Alamos National & 042 & 5470 & FT3 & MIN & 5470 & Carpenter Shop TA-3-38 & 01-Jan-60 & & FALSE \\
\hline Los Alamos National & 043 & 21000 & GAL & DAY & 21000 & Composite Mineral Oil Tank & 01-Jan-86 & & FALSE \\
\hline Los Alamos National & 044 & 10.7 & E6BTU & HR & 12.6 & Boiler TA-50 RLWTF & $01-F e b-00$ & & FALSE \\
\hline Los Alamos National & 045 & 1200 & LB & HR & 1200 & Data Disintegrator TA-52 & 01-Aug-04 & & FALSE \\
\hline
\end{tabular}




\begin{tabular}{|c|c|c|c|c|c|c|c|c|c|c|c|}
\hline Description & $\begin{array}{c}\text { AIRS } \\
\text { Point ID }\end{array}$ & \begin{tabular}{|c} 
AlRS \\
Stack ID \\
\end{tabular} & $\begin{array}{c}\text { State Local } \\
\text { Stack ID }\end{array}$ & \begin{tabular}{|c|} 
Emission \\
Release \\
Point Type \\
\end{tabular} & $\begin{array}{c}\text { Stack } \\
\text { Height }\end{array}$ & $\begin{array}{c}\text { Stack } \\
\text { Diameter }\end{array}$ & $\begin{array}{c}\text { Exit Gas } \\
\text { Temperature } \\
\end{array}$ & $\begin{array}{l}\text { Exit Gas } \\
\text { Velocity }\end{array}$ & UTM Zone X & $x$ Coordinate $Y$ & Y Coordinate \\
\hline Be Machining TA-3 BLDG 39 & 001 & 001 & 001 & 02 & 54 & 1 & 70 & 38 & 13 & 380.48 & 3970.38 \\
\hline TA3-22 Boilers (3 Each) See Pt 32, 33, & 002 & 002 & 002 & 02 & 68 & 8 & 416 & 12 & 13 & 380.5 & 3971 \\
\hline TA16 Boiler (3 Each) - Removed & 003 & 003 & 003 & 02 & 65 & 5 & 0 & 0 & 13 & 378 & 3968 \\
\hline Steam Plant Boiler TA21 BLDG 357 (3) & 004 & 004 & 004 & 02 & 41 & 2 & 268 & 13 & 13 & 385 & 3970.7 \\
\hline TD Site Boiler (Not Built) & 005 & 005 & 005 & 02 & 0 & 0 & 0 & 0 & 13 & 0 & 0 \\
\hline Be Machining TA35 BLDG 213 & 006 & 006 & 006 & 02 & 71 & 1 & 70 & 38 & 13 & 382.85 & 3969.39 \\
\hline Be Machining TA3 BLDG 141 & 007 & 007 & 007 & 02 & 50 & 5 & 70 & 47 & 13 & 381.2 & 3970.28 \\
\hline Be Machining TA3 BLDG 102 & 008 & 008 & 008 & 02 & 45 & 3 & 70 & 19 & 13 & 380.5 & 3970.16 \\
\hline Be Shop TA3-35 (Not Built) & 009 & 009 & 009 & 02 & 0 & 0 & 0 & 0 & 13 & 0 & 0 \\
\hline Be Cutting \& Beand Dressing TA-55-4 & 010 & 010 & 010 & 02 & 32 & 4 & 77 & 30 & 13 & 382 & 3969 \\
\hline Metallography TA55-4 North Stack & 011 & 011 & 011 & 02 & 49 & 4 & 77 & 39 & 13 & 382 & 3969 \\
\hline Solid Waste Fired Boiler (Not Built) & 012 & 012 & 012 & 02 & 0 & 0 & 0 & 0 & 13 & 0 & 0 \\
\hline Asphalt Plant TA-3-73 & 013 & 013 & 013 & 02 & 30 & 4 & 130 & 27 & 13 & 380 & 3970 \\
\hline Caterpillar HCR TA SI Pump Engine & 014 & 014 & 014 & 02 & 17 & 1 & 977 & 75 & 13 & 386 & 3968 \\
\hline Boiler (TA-48-1) BS-1 & 015 & 015 & 015 & 02 & 50 & 2 & 300 & 10 & 13 & 382.13 & 3969.7 \\
\hline Boiler (TA-48-1) BS-2 & 016 & 016 & 016 & 02 & 50 & 2 & 300 & 10 & 13 & 382.13 & 3969.7 \\
\hline Boiler (TA-48-1) BS-6 & 017 & 017 & 017 & 02 & 50 & 2 & 300 & 13 & 13 & 382.13 & 3969.7 \\
\hline Boiler (TA-53-365) BHW-1 & 018 & 018 & 018 & 05 & 22 & 2 & 300 & 0.03 & 13 & 386 & 3969.9 \\
\hline Boiler (TA-53-365) BHW-2 & 019 & 019 & 019 & 05 & 22 & 2 & 300 & 0.03 & 13 & 386 & 3970 \\
\hline Boiler (TA-59-1) BHW-1 & 020 & 020 & 020 & 02 & 55 & 2 & 300 & 19 & 13 & 381 & 3970 \\
\hline Boiler (TA-59-1) BHW-2 & 021 & 021 & 021 & 02 & 55 & 2 & 300 & 19 & 13 & 381 & 3970 \\
\hline Boiler (TA-55-6) BHW-1 & 022 & 022 & 022 & 01 & 30 & 2 & 222 & 24 & 13 & 383 & 3970 \\
\hline Boiler (TA-55-6) BHW-2 & 023 & 023 & 023 & 01 & 30 & 2 & 333 & 29 & 13 & 383 & 3970 \\
\hline Boiler and backup, TA-16, Plant- 5 & 024 & 024 & 024 & 05 & 21 & 2 & 341 & 0.03 & 13 & 379 & 3967.1 \\
\hline Boiler and Backup, Plant 6 (TA-16- & 025 & 025 & 025 & 05 & 19 & 2 & 341 & 0.03 & 13 & 379.28 & 3967.4 \\
\hline Rock Crusher & 026 & 026 & 026 & 02 & 15 & 0.25 & 70 & 0 & 13 & 384.8 & 3970.9 \\
\hline SEM-1424 Disintegrator paper & 027 & 027 & 027 & 05 & 26 & 1 & 70 & 0.03 & 13 & 383.6 & 3969 \\
\hline Degreaser - cold ultrasonic bath TA-55- & 028 & 028 & 028 & 02 & 32 & 4 & 77 & 30 & 13 & 382 & 3969 \\
\hline Degreaser - cold ultrasonic bath TA-46- & 029 & 029 & 029 & 01 & 32 & 1 & 70 & 3 & 13 & 384 & 3968 \\
\hline Degreaser - inhouse cold batch TA-55- & 030 & 030 & 030 & 01 & 32 & 1 & 70 & 3 & 13 & 384 & 3968 \\
\hline Research \& Development Activities - & 031 & 031 & 031 & 01 & 0 & 0 & 0 & 0 & 13 & 384 & 3968 \\
\hline TA3-22 Edgemoor Iron Works Boiler & 032 & 032 & 001 & 02 & 68 & 7.7 & 416 & 57 & 13 & 380.84 & 3970.6 \\
\hline TA3-22 Edgemoor Iron Works Boiler & 033 & 033 & 001 & 02 & 68 & 7.7 & 416 & 57 & 13 & 380.84 & 3970.6 \\
\hline TA3-22 Edgemoor Iron Works Boiler & 034 & 034 & 001 & 02 & 68 & 7.7 & 416 & 29 & 13 & 380.86 & 3970.6 \\
\hline Tank TA-03-026 (No. 2 fuel oil) & 035 & 035 & 035 & 02 & 0 & 0 & 0 & 0 & 13 & 381.19 & 3970.3 \\
\hline Tank TA-03-779 (No. 2 fuel oil) & 036 & 036 & 036 & 02 & 0 & 0 & 0 & 0 & 13 & 381.19 & 3970.3 \\
\hline Sellers Boiler BHW-1B(TA 55, Bldg. & 037 & 037 & $\mathrm{BWH}-1 \mathrm{~B}$ & 05 & 30 & 2 & 334 & 0.03 & 13 & 382.5 & 3969.4 \\
\hline Sellers Boiler BHW-2B(TA 55, Bldg. & 038 & 038 & BWH-2B & 05 & 30 & 2 & 334 & 0.03 & 13 & 382.5 & 3969.4 \\
\hline Air Curtain Destructor S-127 & 039 & 039 & 039 & 01 & 10 & 17 & 2500 & 1 & 13 & 378.10 & 3968.3 \\
\hline Air Curtain Destructor T-350-1 & 040 & 040 & 040 & 01 & 0 & 25 & 2500 & 1 & 13 & 378.1 & 3968.35 \\
\hline Air Curtain Destructor T-350-2 & 041 & 041 & 041 & 01 & 0 & 25 & 2500 & 1 & 13 & 378.1 & 3968.25 \\
\hline Carpenter Shop TA-3-38 & 042 & 042 & 042 & 05 & 20 & 3 & 75 & 0.03 & 13 & 380.26 & 3970.7 \\
\hline Composite Mineral Oil Tank & 043 & 043 & 043 & 02 & 0 & 0 & 0 & 0 & 13 & 386.72 & 3969.92 \\
\hline Boiler TA-50 RLWTF & 044 & 044 & 044 & 05 & 15 & 1.4 & 400 & 0.03 & 13 & 382.85 & 3969.2 \\
\hline Data Disintegrator - TA_52 & 045 & 045 & 045 & 03 & 26 & 0.6 & 70 & 71 & 13 & 383.6 & 3968.9 \\
\hline
\end{tabular}




\begin{tabular}{|c|c|c|c|c|c|c|c|c|c|c|c|c|c|c|c|}
\hline AIRS Stack ID & SCC AFS & $\begin{array}{c}\text { Process } \\
\text { MACT Code }\end{array}$ & Description & \begin{tabular}{|c|}
$\begin{array}{c}\text { Winter } \\
\text { Throughput } \\
\text { PCT }\end{array}$ \\
\end{tabular} & \begin{tabular}{c|} 
Spring \\
Throughput \\
PCT
\end{tabular} & \begin{tabular}{c|c} 
Summer & \\
Throughput & PCT \\
PCT
\end{tabular} & \begin{tabular}{c|c} 
Fall \\
Throughput \\
PCT
\end{tabular} & $\begin{array}{c}\text { Annual Avg } \\
\text { Days Per } \\
\text { Week }\end{array}$ & \begin{tabular}{c|} 
Annual Avg \\
Weeks Per \\
Year
\end{tabular} & \begin{tabular}{|c|}
$\begin{array}{c}\text { Annual Avg } \\
\text { Hours Per } \\
\text { Day }\end{array}$ \\
\end{tabular} & \begin{tabular}{|c|c|}
$\begin{array}{c}\text { Annual Avg } \\
\text { Hours Per } \\
\text { Year }\end{array}$ & C \\
\end{tabular} & $\begin{array}{c}\text { Heat } \\
\text { Content }\end{array}$ & $\begin{array}{c}\text { Sulfur } \\
\text { Content }\end{array}$ & $\mid \begin{array}{c}\text { Ash } \\
\text { Content }\end{array}$ & \begin{tabular}{|c|} 
Process MACT \\
Compliance \\
Status
\end{tabular} \\
\hline 001 & 30903004 & & Be Machining TA-3 BLDG 39 & 0 & 0 & 0 & 0 & 0 & 0 & 0 & 0 & 0 & 0 & 0 & \\
\hline 002 & 10100601 & & TA3-22 Boilers (3 Each) See Pt 32, 33, & 0 & 0 & 0 & 0 & 0 & 0 & 0 & 0 & 0 & 0 & 0 & \\
\hline 003 & 10100602 & & TA16 Boiler (3 Each) - Removed & 0 & 0 & 0 & 0 & 0 & 0 & 0 & 0 & 0 & 0 & 0 & \\
\hline 004 & 10100602 & & Steam Plant Boiler TA21 BLDG 357 (3) & 25 & 25 & 25 & 25 & 7 & 52 & 24 & 8760 & 1030 & 0 & 0 & \\
\hline 005 & 10100602 & & TD Site Boiler (Not Built) & 0 & 0 & 0 & 0 & 0 & 0 & 0 & 0 & 0 & 0 & 0 & \\
\hline 006 & 30903004 & & Be Machining TA35 BLDG 213 & 15 & 20 & 30 & 35 & 7 & 52 & 5 & 1920 & 0 & 0 & 0 & \\
\hline 007 & 30903004 & & Be Machining TA3 BLDG 141 & 25 & 25 & 25 & 25 & 7 & 52 & 24 & 8760 & 0 & 0 & 0 & \\
\hline 008 & 30903004 & & Be Machining TA3 BLDG 102 & 0 & 0 & 0 & 0 & 0 & 0 & 0 & 0 & 0 & 0 & 0 & \\
\hline 009 & 30903004 & & Be Shop TA3-35 (Not Built) & 0 & 0 & 0 & 0 & 0 & 0 & 0 & 0 & 0 & 0 & 0 & \\
\hline 010 & 30903004 & & Be Cutting \& Beand Dressing TA-55-4 & 25 & 25 & 25 & 25 & 7 & 52 & 24 & 8760 & 0 & 0 & 0 & \\
\hline 011 & 30900303 & & Metallography TA55-4 North Stack & 25 & 25 & 25 & 25 & 7 & 52 & 24 & 8760 & 0 & 0 & 0 & \\
\hline 012 & 10301202 & & Solid Waste Fired Boiler (Not Built) & 0 & 0 & 0 & 0 & 0 & 0 & 0 & 0 & 0 & 0 & 0 & \\
\hline 013 & 30500211 & & Asphalt Plant TA-3-73 & 0 & 0 & 0 & 0 & 0 & 0 & 0 & 0 & 0 & 0 & 0 & \\
\hline 014 & 20200202 & & Caterpillar HCR TA SI Pump Engine & 0 & 0 & 0 & 0 & 0 & 0 & 0 & 0 & 0 & 0 & 0 & \\
\hline 015 & 10100602 & & Boiler (TA-48-1) BS-1 & 40 & 20 & 0 & 40 & 7 & 33 & 15 & 5500 & 1030 & 0 & 0 & \\
\hline 016 & 10100602 & & Boiler (TA-48-1) BS-2 & 40 & 20 & 0 & 40 & 7 & 33 & 15 & 5500 & 1030 & 0 & 0 & \\
\hline 017 & 10100602 & & Boiler (TA-48-1) BS-6 & 40 & 20 & 0 & 40 & 7 & 33 & 15 & 5500 & 1030 & 0 & 0 & \\
\hline 018 & 10100602 & & Boiler (TA-53-365) BHW-1 & 40 & 20 & 0 & 40 & 7 & 33 & 15 & 5500 & 1030 & 0 & 0 & \\
\hline 019 & 10100602 & & Boiler (TA-53-365) BHW-2 & 40 & 20 & 0 & 40 & 7 & 33 & 15 & 5500 & 1030 & 0 & 0 & \\
\hline 020 & 10100602 & & Boiler (TA-59-1) BHW-1 & 40 & 20 & 0 & 40 & 7 & 33 & 15 & 5500 & 1030 & 0 & 0 & \\
\hline 021 & 10100602 & & Boiler (TA-59-1) BHW-2 & 40 & 20 & 0 & 40 & 7 & 33 & 15 & 5500 & 1030 & 0 & 0 & \\
\hline 022 & 10100602 & & Boiler (TA-55-6) BHW-1 & 0 & 0 & 0 & 0 & 0 & 0 & 0 & 0 & 0 & 0 & 0 & \\
\hline 023 & 10100602 & & Boiler (TA-55-6) BHW-2 & 0 & 0 & 0 & 0 & 0 & 0 & 0 & 0 & 0 & 0 & 0 & \\
\hline 024 & 10100602 & & Boiler and backup, TA-16, Plant-5 & 25 & 25 & 25 & 25 & 7 & 52 & 24 & 8760 & 1030 & 0 & 0 & \\
\hline 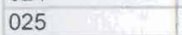 & 10100602 & & Boiler and Backup, Plant 6 (TA-16-1485) & 0 & 0 & 0 & 0 & 0 & 0 & 0 & 0 & 0 & 0 & 0 & \\
\hline 026 & 30502501 & & Rock Crusher & 0 & 0 & 0 & 0 & 0 & 0 & 0 & 0 & 0 & 0 & 0 & \\
\hline 027 & 30701399 & & SEM-1424 Disintegrator paper shredder & 25 & 25 & 25 & 25 & 5 & 52 & 4 & 1040 & 0 & 0 & 0 & \\
\hline 028 & 40100336 & 1614 & Degreaser - cold ultrasonic bath TA-55-4 & 25 & 25 & 25 & 25 & 1 & 52 & 4 & 208 & 0 & 0 & 0 & 03 \\
\hline 029 & 40100336 & 1614 & Degreaser - cold ultrasonic bath TA-46- & 0 & 0 & 0 & 0 & 0 & 0 & 0 & 0 & 0 & 0 & 0 & 03 \\
\hline 030 & 40100336 & 1614 & Degreaser - inhouse cold batch TA-55-4 & 0 & 0 & 0 & 0 & 0 & 0 & 0 & 0 & 0 & 0 & 0 & 03 \\
\hline 031 & 31503001 & & Research \& Development Activities - & 25 & 25 & 25 & 25 & 7 & 52 & 24 & 8760 & 0 & 0 & 0 & \\
\hline 032 & 10100601 & & TA3-22 Edgemoor Iron Works Boiler & 30 & 20 & 20 & 30 & 7 & 52 & 24 & 8760 & 1030 & 0 & 0 & \\
\hline 033 & 10100601 & & TA3-22 Edgemoor Iron Works Boiler & 30 & 20 & 20 & 30 & 7 & 52 & 24 & 8760 & 1030 & 0 & 0 & \\
\hline 034 & 10100601 & & TA3-22 Edgemoor Iron Works Boiler & 30 & 20 & 20 & 30 & 7 & 52 & 24 & 8760 & 1030 & 0 & 0 & \\
\hline 035 & 39090004 & & Tank TA-03-026 (No. 2 fuel oil) & 30 & 20 & 20 & 30 & 7 & 52 & 24 & 8760 & 137 & 0 & 0 & \\
\hline 036 & 39090004 & & Tank TA-03-779 (No. 2 fuel oil) & 30 & 20 & 20 & 30 & 7 & 52 & 24 & 8760 & 137 & 0 & 0 & \\
\hline 037 & 10100602 & & Sellers Boiler BHW-1B(TA 55, Bldg. PF6) & 40 & 20 & 0 & 40 & 7 & 33 & 15 & 5500 & 1030 & 0 & 0 & \\
\hline 038 & 10100602 & & Sellers Boiler BHW-2B(TA 55, Bldg. PF6) & 40 & 20 & 0 & 40 & 7 & 33 & 15 & 5500 & 1030 & 0 & 0 & \\
\hline 039 & 30181001 & & Air Curtain Destructor S-127 & 0 & 0 & 0 & 0 & 0 & 0 & 0 & 0 & 0 & 0 & 0 & \\
\hline 040 & 30181001 & & Air Curtain Destructor T-350-1 & 0 & 0 & 0 & 0 & 0 & 0 & 0 & 0 & 0 & 0 & 0 & \\
\hline 041 & 30181001 & & Air Curtain Destructor T-350-2 & 0 & 0 & 0 & 0 & 0 & 0 & 0 & 0 & 0 & 0 & 0 & \\
\hline 042 & 30700804 & & Carpenter Shop TA-3-38 & 20 & 30 & 30 & 20 & 7 & 52 & 12 & 4368 & 4500 & 0 & 0 & \\
\hline 043 & 39090004 & & Composite Mineral Oil Tank & 25 & 25 & 25 & 25 & 7 & 52 & 24 & 8760 & 0 & 0 & 0 & \\
\hline 044 & 10100602 & & Boiler TA-50 RLWTF & 25 & 25 & 25 & 25 & 2 & 12 & 12 & 192 & 1030 & 0 & 0 & \\
\hline NEW & 30701399 & & Data Disintegrator - TA52 & 25 & 25 & 25 & 25 & 5 & 52 & 6.5 & 2000 & 0 & 0 & 0 & \\
\hline
\end{tabular}




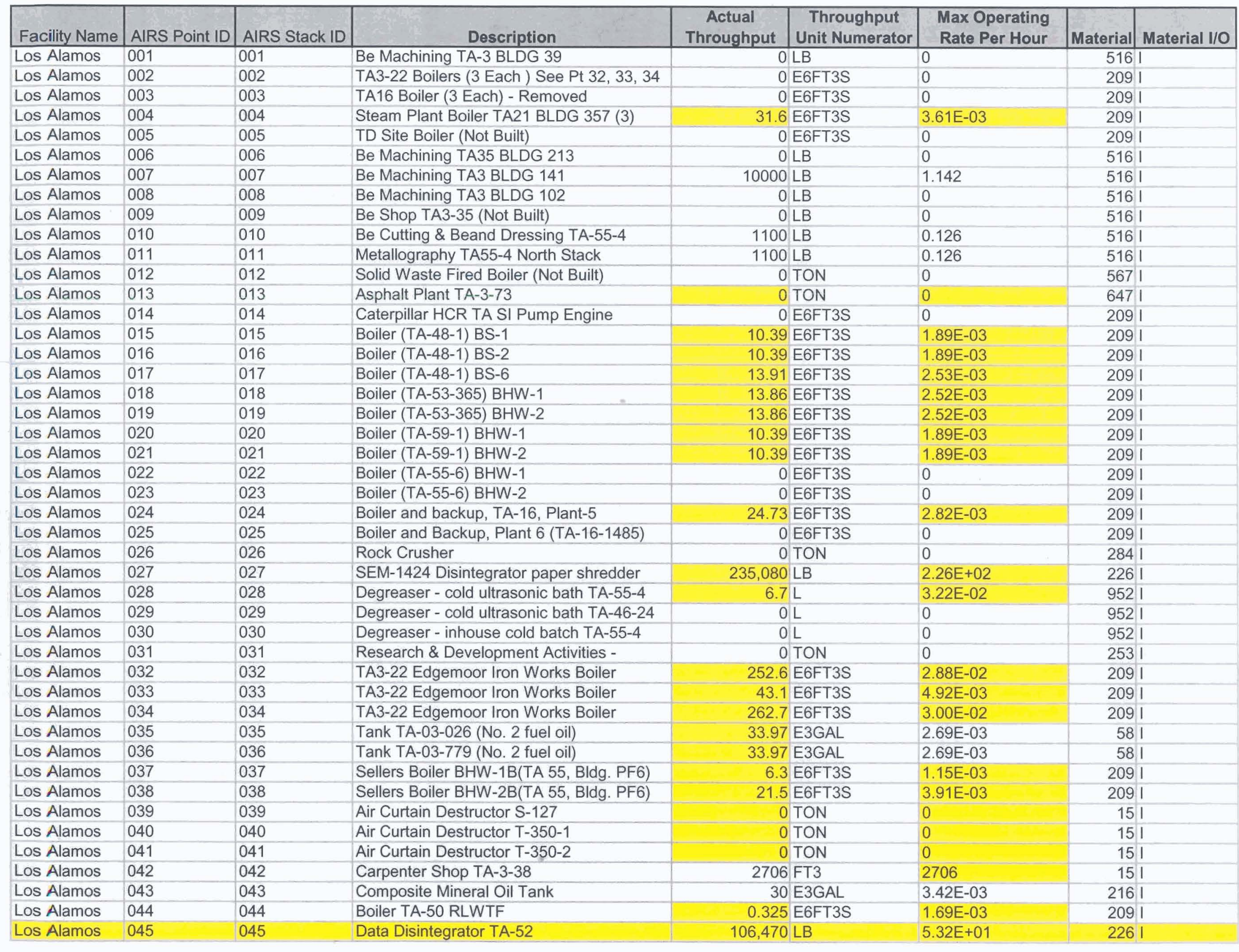




\begin{tabular}{|c|c|c|c|c|c|c|c|c|c|c|}
\hline AIRS Stack ID & Segment ID & $\begin{array}{c}\text { Pollutant } \\
\text { Code }\end{array}$ & CAS Number & $\begin{array}{c}\text { Emission } \\
\text { Numeric Value }\end{array}$ & $\begin{array}{c}\text { Emission } \\
\text { Unit } \\
\text { Numerator }\end{array}$ & $\begin{array}{c}\text { Emission } \\
\text { Type }\end{array}$ & $\begin{array}{c}\text { Calculation } \\
\begin{array}{c}\text { Method } \\
\text { Code }\end{array}\end{array}$ & $\begin{array}{c}\text { Factor } \\
\text { Numeric } \\
\text { Value }\end{array}$ & $\begin{array}{l}\text { Factor Unit } \\
\text { Numerator }\end{array}$ & $\begin{array}{c}\text { Factor Unit } \\
\text { Denominator }\end{array}$ \\
\hline 001 & 01 & 100414 & 100414 & 0 & TY & 12 & 08 & & & \\
\hline 001 & 01 & 110543 & 110543 & 0 & TY & 12 & 08 & & & \\
\hline 001 & 01 & 75070 & 75070 & 0 & TY & 12 & 08 & & & \\
\hline 001 & 01 & 7664393 & 7664393 & 0 & TY & 12 & 08 & & & \\
\hline 001 & 01 & $\mathrm{BE}$ & 7440417 & 0 & TY & 12 & 08 & & & \\
\hline 001 & 01 & $B Z$ & 71432 & 0 & TY & 12 & 08 & & & \\
\hline 001 & 01 & FORM & 50000 & 0 & TY & 12 & 08 & & & \\
\hline 001 & 01 & HC81 & 1330207 & 0 & TY & 12 & 08 & & & \\
\hline 001 & 01 & $\mathrm{NH} 3$ & 7664417 & 0 & TY & 12 & 08 & & & \\
\hline 001 & 01 & PM10 & & 0 & TY & 12 & 08 & & & \\
\hline 001 & 01 & PM25 & & 0 & TY & 12 & 08 & & & \\
\hline 001 & 01 & PT & & 0 & TY & 12 & 08 & & & \\
\hline 001 & 01 & TOLU & 108883 & 0 & TY & 12 & 08 & & & \\
\hline 002 & 01 & $\mathrm{CO}$ & 630080 & 0 & TY & 12 & 08 & & & \\
\hline 002 & 01 & $\mathrm{NH} 3$ & 7664417 & 0 & TY & 12 & 08 & & & \\
\hline 002 & 01 & NO2 & 10102440 & 0 & TY & 12 & 08 & & & \\
\hline 002 & 01 & PM10 & & 0 & TY & 12 & 08 & & & \\
\hline 002 & 01 & PM25 & & 0 & TY & 12 & 08 & & & \\
\hline 002 & 01 & PT & & 0 & TY & 12 & 08 & & & \\
\hline 002 & 01 & $\mathrm{SO} 2$ & 7446095 & 0 & TY & 12 & 08 & & & \\
\hline 002 & 01 & VOC & & 0 & TY & 12 & 08 & & & \\
\hline 003 & 01 & $\mathrm{NO} 2$ & 10102440 & 0 & TY & 12 & 00 & & & \\
\hline 004 & 01 & 106467 & & 1.89E-05 & TY & 12 & 08 & 0.0012 & LB & E6FT3S \\
\hline 004 & 01 & 109 & & 2.07E-07 & TY & 12 & 08 & 0.000012 & LB & E6FT3S \\
\hline 004 & 01 & 110543 & 110543 & 2.84E-02 & TY & 12 & 08 & 1.8 & LB & E6FT3S \\
\hline 004 & 01 & 125 & 10102440 & $1.74 \mathrm{E}-05$ & TY & 12 & 08 & 0.0011 & LB & E6FT3S \\
\hline 004 & 01 & 136 & 136 & $2.21 E-05$ & TY & 12 & 08 & 0.0014 & LB & E6FT3S \\
\hline 004 & 01 & 139 & 139 & 1.33E-06 & TY & 12 & 08 & 0.000084 & LB & E6FT3S \\
\hline 004 & 01 & 195 & 195 & 7.94E-06 & TY & 12 & 08 & 0.0005 & LB & E6FT3S \\
\hline 004 & 01 & 198 & 198 & $6.03 E-06$ & TY & 12 & 08 & 0.00038 & LB & E6FT3S \\
\hline 004 & 01 & 199 & 199 & 4.12E-06 & TY & 12 & 08 & 0.00026 & LB & E6FT3S \\
\hline 004 & 01 & 226 & 226 & $3.32 E-05$ & TY & 12 & 08 & 0.0021 & LB & E6FT3S \\
\hline 004 & 01 & 246 & & $1.53 E-06$ & TY & 12 & 08 & 0.000088 & LB & E6FT3S \\
\hline 004 & 01 & 253 & 253 & 4.66E-07 & TY & 12 & 08 & 0.000024 & LB & E6FT3S \\
\hline 004 & 01 & 91203 & & 9.63E-06 & TY & 12 & 08 & 0.00061 & LB & E6FT3S \\
\hline 004 & 01 & 93 & & $3.18 \mathrm{E}-06$ & TY & 12 & 08 & 0.0002 & LB & E6FT3S \\
\hline 004 & 01 & $B Z$ & 71432 & $3.31 E-05$ & TY & 12 & 08 & 0.0021 & LB & E6FT3S \\
\hline 004 & 01 & $\mathrm{CO}$ & 630080 & 1. $33 E+00$ & TY & 12 & 08 & 84 & LB & E6FT3S \\
\hline 004 & 01 & FORM & 50000 & $1.19 \mathrm{E}-03$ & TY & 12 & 08 & 0.075 & LB & E6FT3S \\
\hline 004 & 01 & $\mathrm{NH} 3$ & 7664417 & & TY & 12 & 00 & & & \\
\hline 004 & 01 & NO2 & 10102440 & $1.58 \mathrm{E}+00$ & TY & 12 & 08 & 100 & LB & E6FT3S \\
\hline 004 & 01 & PM10 & & 1.20E-01 & TY & 12 & 08 & 7.6 & LB & E6FT3S \\
\hline 004 & 01 & PM25 & & 1.20E-01 & TY & 12 & 08 & 7.6 & LB & E6FT3S \\
\hline 004 & 01 & PT & & 1.20E-01 & TY & 12 & 08 & 7.6 & LB & E6FT3S \\
\hline 004 & 01 & $\mathrm{SO} 2$ & 7446095 & $1.20 \mathrm{E}-02$ & TY & 12 & 08 & 0.6 & LB & E6FT3S \\
\hline 004 & 01 & TOLU & 108883 & 5.37E-05 & TY & 12 & 08 & 0.0034 & LB & E6FT3S \\
\hline 004 & 01 & VOC & & $8.70 \mathrm{E}-02$ & TY & 12 & 08 & 5.5 & LB & E6FT3S \\
\hline 005 & 01 & $\mathrm{CO}$ & 630080 & 0 & TY & 12 & 08 & & & \\
\hline 005 & 01 & $\mathrm{NH} 3$ & 7664417 & 0 & TY & 12 & 08 & & & \\
\hline 005 & 01 & $\mathrm{NO} 2$ & 10102440 & 0 & TY & 12 & 08 & & & \\
\hline 005 & 01 & PM10 & & 0 & TY & 12 & 08 & & & \\
\hline 005 & 01 & PM25 & & 0 & TY & 12 & 08 & & & \\
\hline 005 & 01 & PT & & 0 & TY & 12 & 08 & & & \\
\hline 005 & 01 & VOC & & 0 & TY & 12 & 08 & & & \\
\hline 006 & 01 & $\mathrm{BE}$ & 7440417 & $1.98 \mathrm{E}-08$ & TY & 12 & 08 & & & \\
\hline 006 & 01 & $\mathrm{NH} 3$ & 7664417 & & TY & 12 & 00 & & & \\
\hline 006 & 01 & PM10 & & $1.98 \mathrm{E}-08$ & TY & 12 & 08 & & & \\
\hline 006 & 01 & PM25 & & $1.98 E-08$ & TY & 12 & 08 & & & \\
\hline 006 & 01 & PT & & $1.98 \mathrm{E}-08$ & TY & 12 & 08 & & & \\
\hline 007 & 01 & $B E$ & 7440417 & $4.00 E-09$ & TY & 12 & 08 & & & \\
\hline 007 & 01 & $\mathrm{NH} 3$ & 7664417 & & TY & 12 & 00 & & & \\
\hline 007 & 01 & PM10 & & $4.00 E-09$ & TY & 12 & 08 & & & \\
\hline 007 & 01 & PM25 & & 4.00E-09 & TY & 12 & 08 & & & \\
\hline 007 & 01 & PT & & $4.00 E-09$ & TY & 12 & 08 & & & \\
\hline 008 & 01 & $\mathrm{BE}$ & 7440417 & 0 & TY & 12 & 08 & & & \\
\hline 008 & 01 & $\mathrm{NH} 3$ & 7664417 & & TY & 12 & 00 & & & \\
\hline 008 & 01 & PM10 & & 0 & TY & 12 & 08 & & & \\
\hline 008 & 01 & PM25 & & 0 & TY & 12 & 08 & & & \\
\hline 008 & 01 & PT & & 0 & TY & 12 & 08 & & & \\
\hline 009 & 01 & $B E$ & 7440417 & 0 & TY & 12 & 08 & & & \\
\hline 009 & 01 & $\mathrm{NH} 3$ & 7664417 & & TY & 12 & 00 & & & \\
\hline 009 & 01 & PM10 & & 0 & TY & 12 & 08 & & & \\
\hline 009 & 01 & PM25 & & 0 & TY & 12 & 08 & & & \\
\hline 009 & 01 & PT & & 0 & TY & 12 & 08 & & & \\
\hline 010 & 01 & AL-PT & 7429905 & $1.56 \mathrm{E}-06$ & TY & 12 & 08 & & & \\
\hline 010 & 01 & $\mathrm{BE}$ & 7440417 & 1.56E-06 & TY & 12 & 08 & & & \\
\hline 010 & 01 & $\mathrm{NH} 3$ & 7664417 & & TY & 12 & 00 & & & \\
\hline
\end{tabular}




\begin{tabular}{|c|c|c|c|c|c|c|c|c|}
\hline 010 & 01 & PM10 & & 1.56E-06 TY & 12 & 08 & & \\
\hline 010 & 01 & PM25 & & 1.56E-06 TY & 12 & 08 & & \\
\hline 011 & 01 & AL-PT & 7440417 & 1.56E-06 TY & 12 & 08 & & \\
\hline 011 & 01 & $\mathrm{BE}$ & 7440417 & 1.56E-06 TY & 12 & 08 & & \\
\hline 011 & 01 & $\mathrm{NH} 3$ & 7664417 & TY & 12 & 00 & & \\
\hline 011 & 01 & PM10 & & 1.56E-06 TY & 12 & 08 & & \\
\hline 011 & 01 & PM25 & & 1.56E-06 TY & 12 & 08 & & \\
\hline 012 & 01 & PM10 & & OTY & 12 & 00 & & \\
\hline 013 & 01 & 100414 & 100414 & OTY & 12 & 08 & & \\
\hline 013 & 01 & 106514 & 106514 & OTY & 12 & 00 & & \\
\hline 013 & 01 & 110543 & 110543 & TY & 12 & 00 & & \\
\hline 013 & 01 & 246 & & OTY & 12 & 00 & & \\
\hline 013 & 01 & 75070 & 75070 & OTY & 12 & 08 & & \\
\hline 013 & 01 & 7664393 & 7664393 & TY & 12 & 00 & 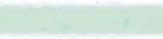 & \\
\hline 013 & 01 & 91203 & 91203 & OTY & 12 & 00 & & \\
\hline 013 & 01 & $\mathrm{BZ}$ & 71432 & OTY & 12 & 08 & & \\
\hline 013 & 01 & $\mathrm{CO}$ & 630080 & OTY & 12 & 08 & & \\
\hline 013 & 01 & FORM & 50000 & OTY & 12 & 08 & & \\
\hline 013 & 01 & $\mathrm{HC} 81$ & 1330207 & OTY & 12 & 08 & & \\
\hline 013 & 01 & $\mathrm{NH} 3$ & 7664417 & TY & 12 & 00 & & \\
\hline 013 & 01 & NO2 & 10102440 & OTY & 12 & 08 & & \\
\hline 013 & 01 & PM10 & & OTY & 12 & 08 & & \\
\hline 013 & 01 & PM25 & & OTY & 12 & 08 & & \\
\hline 013 & 01 & PT & & OTY & 12 & 08 & & \\
\hline 013 & 01 & $\mathrm{SO} 2$ & 7446095 & OTY & 12 & 08 & & \\
\hline 013 & 01 & TOLU & 108883 & OTY & 12 & 08 & & \\
\hline 013 & 01 & VOC & & OTY & 12 & 08 & & \\
\hline 014 & 01 & 100414 & 100414 & OTY & 12 & 08 & & \\
\hline 014 & 01 & 110543 & 110543 & OTY & 12 & 08 & & \\
\hline 014 & 01 & 75070 & 75070 & OTY & 12 & 08 & & \\
\hline 014 & 01 & 7664393 & 7664393 & OTY & 12 & 08 & & \\
\hline 014 & 01 & $B Z$ & 71432 & OTY & 12 & 08 & & \\
\hline 014 & 01 & $\mathrm{CO}$ & 630080 & OTY & 12 & 08 & & \\
\hline 014 & 01 & FORM & 50000 & OTY & 12 & 08 & & \\
\hline 014 & 01 & HC81 & 1330207 & OTY & 12 & 08 & & \\
\hline 014 & 01 & $\mathrm{NH} 3$ & 7664417 & OTY & 12 & 08 & & \\
\hline 014 & 01 & $\mathrm{NO} 2$ & 10102440 & OTY & 12 & 08 & & \\
\hline 014 & 01 & PM10 & & 0 TY & 12 & 08 & & \\
\hline 014 & 01 & PM25 & & $0 T Y$ & 12 & 08 & & \\
\hline 014 & 01 & PT & & $0 T Y$ & 12 & 08 & & \\
\hline 014 & 01 & $\mathrm{SO} 2$ & 7446095 & OTY & 12 & 08 & & \\
\hline 014 & 01 & TOLU & 108883 & $0 T Y$ & 12 & 08 & & \\
\hline 014 & 01 & VOC & & OTY & 12 & 08 & & \\
\hline 015 & 01 & 100414 & 100414 & TY & 12 & 00 & & \\
\hline 015 & 01 & 106467 & & 6.24E-06 TY & 12 & 08 & 0.0012 LB & E6FT3S \\
\hline 015 & 01 & 109 & 630080 & 6.24E-08 TY & 12 & 08 & 0.000012 LB & E6FT3S \\
\hline 015 & 01 & 110543 & 110543 & $9.35 \mathrm{E}-03 \mathrm{TY}$ & 12 & 08 & $1.8 \mathrm{LB}$ & E6FT3S \\
\hline 015 & 01 & 125 & 630080 & $5.72 E-06$ TY & 12 & 08 & 0.0011 LB & E6FT3S \\
\hline 015 & 01 & 136 & 136 & $7.28 \mathrm{E}-06 \mathrm{TY}$ & 12 & 08 & 0.0014 LB & E6FT3S \\
\hline 015 & 01 & 139 & 139 & 4.37E-07 TY & 12 & 08 & 0.000084 LB & E6FT3S \\
\hline 015 & 01 & 195 & 195 & 2.60E-06 TY & 12 & 08 & 0.0005 LB & E6FT3S \\
\hline 015 & 01 & 198 & 198 & 1.97E-06 TY & 12 & 08 & 0.00038 LB & E6FT3S \\
\hline 015 & 01 & 199 & 199 & 1.35E-06 TY & 12 & 08 & 0.00026 LB & E6FT3S \\
\hline 015 & 01 & 226 & 226 & 1.09E-05 TY & 12 & 08 & 0.0021 LB & E6FT3S \\
\hline 015 & 01 & 246 & & 4.57E-07 TY & 12 & 08 & 0.000088 LB & E6FT3S \\
\hline 015 & 01 & 253 & 253 & $1.25 E-07$ TY & 12 & 08 & 0.000024 LB & E6FT3S \\
\hline 015 & 01 & 75070 & 75070 & TY & 12 & 00 & (x) & \\
\hline 015 & 01 & 7664393 & 7664393 & TY & 12 & 00 & $45=x-x=$ & \\
\hline 015 & 01 & 91203 & & 3.17E-06 TY & 12 & 08 & 0.00061 LB & E6FT3S \\
\hline 015 & 01 & 93 & 630080 & 1.04E-06 TY & 12 & 08 & 0.0002 LB & E6FT3S \\
\hline 015 & 01 & $B Z$ & 71432 & 1.09E-05 TY & 12 & 08 & 0.0021 LB & E6FT3S \\
\hline 015 & 01 & $\mathrm{CO}$ & 630080 & 4.37E-01 TY & 12 & 08 & 84 LB & E6FT3S \\
\hline 015 & 01 & FORM & 50000 & 3.90E-04 TY & 12 & 08 & 0.075 LB & E6FT3S \\
\hline 015 & 01 & $\mathrm{HC} 81$ & 1330207 & TY & 12 & 00 & & \\
\hline 015 & 01 & $\mathrm{NH} 3$ & 7664417 & TY & 12 & 00 & & \\
\hline 015 & 01 & NO2 & 10102440 & 5.20E-01 TY & 12 & 08 & 100 LB & E6FT3S \\
\hline 015 & 01 & PM10 & & 3.95E-02 TY & 12 & 08 & $7.6 \mathrm{LB}$ & E6FT3S \\
\hline 015 & 01 & PM25 & & $3.95 \mathrm{E}-02 \mathrm{TY}$ & 12 & 08 & $7.6 \mathrm{LB}$ & E6FT3S \\
\hline 015 & 01 & PT & & 3.95E-02 TY & 12 & 08 & $7.6 \mathrm{LB}$ & E6FT3S \\
\hline 015 & 01 & $\mathrm{SO} 2$ & 7446095 & $3.12 \mathrm{E}-03 \mathrm{TY}$ & 12 & 08 & $0.6 \mathrm{LB}$ & E6FT3S \\
\hline 015 & 01 & TOLU & 108883 & 1.77E-05 TY & 12 & 08 & 0.0034 LB & E6FT3S \\
\hline 015 & 01 & VOC & & $2.86 \mathrm{E}-02 \mathrm{TY}$ & 12 & 08 & 5.5 LB & E6FT3S \\
\hline 016 & 01 & 100414 & 100414 & TY & 12 & 00 & & \\
\hline 016 & 01 & 106467 & & 6.23E-06 TY & 12 & 08 & 0.0012 LB & E6FT3S \\
\hline 016 & 01 & 109 & & $6.23 E-08 T Y$ & 12 & 08 & 0.000012 LB & E6FT3S \\
\hline 016 & 01 & 110543 & 110543 & 9.35E-03 TY & 12 & 08 & $1.8 \mathrm{LB}$ & E6FT3S \\
\hline 016 & 01 & 125 & & 5.72E-06 TY & 12 & 08 & 0.0011 LB & E6FT3S \\
\hline 016 & 01 & 136 & 136 & 7.27E-06 TY & 12 & 08 & 0.0014 LB & E6FT3S \\
\hline 016 & 01 & 139 & 139 & 4.36E-07 TY & 12 & 08 & 0.000084 LB & E6FT3S \\
\hline 016 & 01 & 195 & 195 & 2.60E-06 TY & 12 & 08 & 0.0005 LB & E6FT3S \\
\hline
\end{tabular}




\begin{tabular}{|c|c|c|c|c|c|c|c|c|c|}
\hline 016 & 01 & 198 & 198 & 1.97E-06 T & TY & 12 & 08 & 0.00038 LB & E6FT3S \\
\hline 016 & 01 & 199 & 199 & 1.35E-06 T & TY & 12 & 08 & 0.00026 LB & E6FT3S \\
\hline 016 & 01 & 226 & 226 & 1.09E-05 T & TY & 12 & 08 & 0.0021 LB & E6FT3S \\
\hline 016 & 01 & 246 & & 4.57E-07 T & TY & 12 & 08 & 0.000088 LB & E6FT3S \\
\hline 016 & 01 & 253 & 253 & 1.25E-07 T & TY & 12 & 08 & 0.000024 LB & E6FT3S \\
\hline 016 & 01 & 75070 & 75070 & & TY & 12 & 00 & & \\
\hline 016 & 01 & 7664393 & 7664393 & & TY & 12 & 00 & & \\
\hline 016 & 01 & 91203 & & 3.17E-06 T & TY & 12 & 08 & 0.00061 LB & E6FT3S \\
\hline 016 & 01 & 93 & & 1.04E-06 T & TY & 12 & 08 & 0.0002 LB & E6FT3S \\
\hline 016 & 01 & $B Z$ & 71432 & 1.09E-05 T & TY & 12 & 08 & 0.0021 LB & E6FT3S \\
\hline 016 & 01 & $\mathrm{CO}$ & 630080 & 4.36E-01 T & TY & 12 & 08 & 84 LB & E6FT3S \\
\hline 016 & 01 & FORM & 50000 & $3.90 \mathrm{E}-04 \mathrm{~T}$ & TY & 12 & 08 & 0.075 LB & E6FT3S \\
\hline 016 & 01 & HC81 & 1330207 & & TY & 12 & 00 & & \\
\hline 016 & 01 & $\mathrm{NH} 3$ & 7664417 & & TY & 12 & 00 & & \\
\hline 016 & 01 & NO2 & 10102440 & 5.20E-01 T & TY & 12 & 08 & 100 LB & E6FT3S \\
\hline 016 & 01 & PM10 & & 3.95E-02 T & TY & 12 & 08 & $7.6 \mathrm{LB}$ & E6FT3S \\
\hline 016 & 01 & PM25 & & 3.95E-02 T & TY & 12 & 08 & 7.6 LB & E6FT3S \\
\hline 016 & 01 & PT & & $3.95 \mathrm{E}-02 \mathrm{~T}$ & TY & 12 & 08 & 7.6 LB & E6FT3S \\
\hline 016 & 01 & $\mathrm{SO} 2$ & 7446095 & $3.12 E-03 T$ & TY & 12 & 08 & 0.6 LB & E6FT3S \\
\hline 016 & 01 & TOLU & 108883 & 1.77E-05 T & TY & 12 & 08 & 0.0034 LB & E6FT3S \\
\hline 016 & 01 & VOC & & 2.86E-02 T & TY & 12 & 08 & 5.5 LB & E6FT3S \\
\hline 017 & 01 & 100414 & 100414 & & TY & 12 & 00 & & \\
\hline 017 & 01 & 106467 & & 8.34E-06 T & TY & 12 & 08 & 0.0012 LB & E6FT3S \\
\hline 017 & 01 & 109 & & 8.34E-08 T & TY & 12 & 08 & 0.000012 LB & E6FT3S \\
\hline 017 & 01 & 110543 & 110543 & 1.25E-02 T & TY & 12 & 08 & $1.8 \mathrm{LB}$ & E6FT3S \\
\hline 017 & 01 & 125 & & 7.65E-06 T & TY & 12 & 08 & 0.0011 LB & E6FT3S \\
\hline 017 & 01 & 136 & 136 & $9.73 \mathrm{E}-06 \mathrm{~T}$ & TY & 12 & 08 & 0.0014 LB & E6FT3S \\
\hline 017 & 01 & 139 & 139 & 5.84E-07 T & TY & 12 & 08 & 0.000084 LB & E6FT3S \\
\hline 017 & 01 & 195 & 195 & $3.48 \mathrm{E}-06 \mathrm{~T}$ & TY & 12 & 08 & 0.0005 LB & E6FT3S \\
\hline 017 & 01 & 198 & 198 & 2.64E-06 T & TY & 12 & 08 & 0.00038 LB & E6FT3S \\
\hline 017 & 01 & 199 & 199 & 1.81E-06 T & TY & 12 & 08 & 0.00026 LB & E6FT3S \\
\hline 017 & 01 & 226 & 226 & $1.46 \mathrm{E}-05 \mathrm{~T}$ & TY & 12 & 08 & 0.0021 LB & E6FT3S \\
\hline 017 & 01 & 246 & & 6.12E-07 T & TY & 12 & 08 & 0.000088 LB & E6FT3S \\
\hline 017 & 01 & 253 & 253 & 1.67E-07 T & TY & 12 & 08 & 0.000024 LB & E6FT3S \\
\hline 017 & 01 & 75070 & 75070 & & TY & 12 & 00 & & \\
\hline 017 & 01 & 7664393 & 7664393 & & TY & 12 & 00 & & \\
\hline 017 & 01 & 91203 & & 4.24E-06 T & TY & 12 & 08 & 0.00061 LB & E6FT3S \\
\hline 017 & 01 & 93 & & 1.39E-06 T & TY & 12 & 08 & 0.0002 LB & E6FT3S \\
\hline 017 & 01 & $B Z$ & 71432 & 1.46E-05 T & TY & 12 & 08 & 0.0021 LB & E6FT3S \\
\hline 017 & 01 & $\mathrm{CO}$ & 630080 & 5.84E-01 T & TY & 12 & 08 & 84 LB & E6FT3S \\
\hline 017 & 01 & FORM & 50000 & 5.21E-04 T & TY & 12 & 08 & 0.075 LB & E6FT3S \\
\hline 017 & 01 & HC81 & 1330207 & & TY & 12 & 00 & & \\
\hline 017 & 01 & $\mathrm{NH} 3$ & 7664417 & & TY & 12 & 00 & & \\
\hline 017 & 01 & $\mathrm{NO} 2$ & 10102440 & $6.95 \mathrm{E}-01 \mathrm{~T}$ & TY & 12 & 08 & 100 LB & E6FT3S \\
\hline 017 & 01 & PM10 & & 5.28E-02 T & TY & 12 & 08 & $7.6 \mathrm{LB}$ & E6FT3S \\
\hline 017 & 01 & PM25 & & $5.28 E-02 T$ & TY & 12 & 08 & 7.6 LB & E6FT3S \\
\hline 017 & 01 & PT & & $5.28 \mathrm{E}-02 \mathrm{~T}$ & TY & 12 & 08 & 7.6 LB & E6FT3S \\
\hline 017 & 01 & $\mathrm{SO} 2$ & 7446095 & 4.17E-03 T & TY & 12 & 08 & $0.6 \mathrm{LB}$ & E6FT3S \\
\hline 017 & 01 & TOLU & 108883 & 2.36E-05 T & TY & 12 & 08 & 0.0034 LB & E6FT3S \\
\hline 017 & 01 & VOC & & $3.82 \mathrm{E}-02 \mathrm{~T}$ & TY & 12 & 08 & $5.5 \mathrm{LB}$ & E6FT3S \\
\hline 018 & 01 & 100414 & 100414 & & TY & 12 & 00 & & \\
\hline 018 & 01 & 106467 & & 8.31E-06 T & TY & 12 & 08 & 0.0012 LB & E6FT3S \\
\hline 018 & 01 & 109 & & 8.31E-08 T & TY & 12 & 08 & 0.000012 LB & E6FT3S \\
\hline 018 & 01 & 110543 & 110543 & 1.25E-02 T & TY & 12 & 08 & $1.8 \mathrm{LB}$ & E6FT3S \\
\hline 018 & 01 & 125 & & 7.62E-06 T & TY & 12 & 08 & 0.0011 LB & E6FT3S \\
\hline 018 & 01 & 136 & 136 & $9.70 \mathrm{E}-06 \mathrm{~T}$ & TY & 12 & 08 & 0.0014 LB & E6FT3S \\
\hline 018 & 01 & 139 & 139 & 5.82E-07 T & TY & 12 & 08 & 0.000084 LB & E6FT3S \\
\hline 018 & 01 & 195 & 195 & 3.46E-06 T & TY & 12 & 08 & 0.0005 LB & E6FT3S \\
\hline 018 & 01 & 198 & 198 & 2.63E-06 T & TY & 12 & 08 & 0.00038 LB & E6FT3S \\
\hline 018 & 01 & 199 & 199 & 1.80E-06 T & TY & 12 & 08 & 0.00026 LB & E6FT3S \\
\hline 018 & 01 & 226 & 226 & 1.45E-05 T & TY & 12 & 08 & 0.0021 LB & E6FT3S \\
\hline 018 & 01 & 246 & & 6.10E-07 T & TY & 12 & 08 & 0.000088 LB & E6FT3S \\
\hline 018 & 01 & 253 & 253 & $1.66 \mathrm{E}-07$ & TY & 12 & 08 & 0.000024 LB & E6FT3S \\
\hline 018 & 01 & 75070 & 75070 & & TY & 12 & 00 & & \\
\hline 018 & 01 & 7664393 & 7664393 & & TY & 12 & 00 & & \\
\hline 018 & 01 & 91203 & & 4.23E-06 7 & TY & 12 & 08 & 0.00061 LB & E6FT3S \\
\hline 018 & 01 & 93 & & 1.39E-06 1 & TY & 12 & 08 & 0.0002 LB & E6FT3S \\
\hline 018 & 01 & $B Z$ & 71432 & $1.45 E-05$ & TY & 12 & 08 & 0.0021 LB & E6FT3S \\
\hline 018 & 01 & $\mathrm{CO}$ & 630080 & 5.82E-01 & TY & 12 & 08 & 84 LB & E6FT3S \\
\hline 018 & 01 & FORM & 50000 & $5.20 \mathrm{E}-04$ & TY & 12 & 08 & 0.075 LB & E6FT3S \\
\hline 018 & 01 & HC81 & 1330207 & & TY & 12 & 00 & & \\
\hline 018 & 01 & $\mathrm{NH} 3$ & 7664417 & & TY & 12 & 00 & & \\
\hline 018 & 01 & NO2 & 10102440 & 6.93E-01 & TY & 12 & 08 & 100 LB & E6FT3S \\
\hline 018 & 01 & PM10 & & 5.27E-02 & TY & 12 & 08 & $7.6 \mathrm{LB}$ & E6FT3S \\
\hline 018 & 01 & PM25 & & $5.27 \mathrm{E}-02$ & TY & 12 & 08 & 7.6 LB & E6FT3S \\
\hline 018 & 01 & PT & & $5.27 \mathrm{E}-02$ & TY & 12 & 08 & 7.6 LB & E6FT3S \\
\hline 018 & 01 & $\mathrm{SO} 2$ & 7446095 & 4.16E-03 & TY & 12 & 08 & $0.6 \mathrm{LB}$ & E6FT3S \\
\hline 018 & 01 & TOLU & 108883 & $2.36 \mathrm{E}=05$ & TY & 12 & 08 & 0,0034 LB & E6FT3S \\
\hline 018 & 01 & VOC & & $3.81 \mathrm{E}-02$ & TY & 12 & 08 & $5.5 \mathrm{LB}$ & E6FT3S \\
\hline 019 & 01 & 106467 & 10102440 & 8.31E-06 & TY & 12 & 08 & 0.0012 LB & E6FT3S \\
\hline
\end{tabular}

2004 EI Unit Emissions 


\begin{tabular}{|c|c|c|c|c|c|c|c|c|}
\hline 019 & 01 & 109 & 10102440 & 8.31E-08 TY & 12 & 08 & 0.000012 LB & E6FT3S \\
\hline 019 & 01 & 110543 & 110543 & 1.25E-02 TY & 12 & 08 & $1.8 \mathrm{LB}$ & E6FT3S \\
\hline 019 & 01 & 125 & 10102440 & 7.62E-06 TY & 12 & 08 & 0.0011 LB & E6FT3S \\
\hline 019 & 01 & 136 & 136 & 9.70E-06 TY & 12 & 08 & 0.0014 LB & E6FT3S \\
\hline 019 & 01 & 139 & 139 & 5.82E-07 TY & 12 & 08 & 0.000084 LB & E6FT3S \\
\hline 019 & 01 & 195 & 195 & $3.46 \mathrm{E}-06 \mathrm{TY}$ & 12 & 08 & 0.0005 LB & E6FT3S \\
\hline 019 & 01 & 198 & 198 & 2.63E-06 TY & 12 & 08 & 0.00038 LB & E6FT3S \\
\hline 019 & 01 & 199 & 199 & 1.80E-06 TY & 12 & 08 & 0.00026 LB & E6FT3S \\
\hline 019 & 01 & 226 & 226 & 1.45E-05 TY & 12 & 08 & 0.0021 LB & E6FT3S \\
\hline 019 & 01 & 246 & 10102440 & 6.10E-07 TY & 12 & 08 & 0.000088 LB & E6FT3S \\
\hline 019 & 01 & 253 & 253 & 1.66E-07 TY & 12 & 08 & 0.000024 LB & E6FT3S \\
\hline 019 & 01 & 91203 & & 4.23E-06 TY & 12 & 08 & 0.00061 LB & E6FT3S \\
\hline 019 & 01 & 93 & 10102440 & 1.39E-06 TY & 12 & 08 & 0.0002 LB & E6FT3S \\
\hline 019 & 01 & $B Z$ & 71432 & 1.45E-05 TY & 12 & 08 & 0.0021 LB & E6FT3S \\
\hline 019 & 01 & $\mathrm{CO}$ & 630080 & 5.82E-01 TY & 12 & 08 & 84 LB & E6FT3S \\
\hline 019 & 01 & FORM & 50000 & $5.20 \mathrm{E}-04 \mathrm{TY}$ & 12 & 08 & 0.075 LB & E6FT3S \\
\hline 019 & 01 & $\mathrm{NH} 3$ & 7664417 & TY & 12 & 00 & & \\
\hline 019 & 01 & NO2 & 10102440 & 6.93E-01 TY & 12 & 08 & 100 LB & E6FT3S \\
\hline 019 & 01 & PM10 & & 5.27E-02 TY & 12 & 08 & 7.6 LB & E6FT3S \\
\hline 019 & 01 & PM25 & & 5.27E-02 TY & 12 & 08 & $7.6 \mathrm{LB}$ & E6FT3S \\
\hline 019 & 01 & PT & & 5.27E-02 TY & 12 & 08 & $7.6 \mathrm{LB}$ & E6FT3S \\
\hline 019 & 01 & $\mathrm{SO} 2$ & 7446095 & 4.16E-03 TY & 12 & 08 & $0.6 \mathrm{LB}$ & E6FT3S \\
\hline 019 & 01 & TOLU & 108883 & 2.36E-05 TY & 12 & 08 & 0.0034 LB & E6FT3S \\
\hline 019 & 01 & VOC & & $3.81 E-02 T Y$ & 12 & 08 & 5.5 LB & E6FT3S \\
\hline 020 & 01 & 106467 & & 6.23E-06 TY & 12 & 08 & 0.0012 LB & E6FT3S \\
\hline 020 & 01 & 109 & & $6.23 E-08$ TY & 12 & 08 & 0.000012 LB & E6FT3S \\
\hline 020 & 01 & 110543 & 110543 & 9.35E-03 TY & 12 & 08 & $1.8 \mathrm{LB}$ & E6FT3S \\
\hline 020 & 01 & 125 & & $5.72 E-06 \mathrm{TY}$ & 12 & 08 & 0.0011 LB & E6FT3S \\
\hline 020 & 01 & 136 & 136 & 7.27E-06 TY & 12 & 08 & 0.0014 LB & E6FT3S \\
\hline 020 & 01 & 139 & 139 & 4.36E-07 TY & 12 & 08 & 0.000084 LB & E6FT3S \\
\hline 020 & 01 & 195 & 195 & $2.60 \mathrm{E}-06 \mathrm{TY}$ & 12 & 08 & 0.0005 LB & E6FT3S \\
\hline 020 & 01 & 198 & 198 & 1.97E-06 TY & 12 & 08 & 0.00038 LB & E6FT3S \\
\hline 020 & 01 & 199 & 199 & 1.35E-06 TY & 12 & 08 & 0.00026 LB & E6FT3S \\
\hline 020 & 01 & 226 & 226 & 1.09E-05 TY & 12 & 08 & 0.0021 LB & E6FT3S \\
\hline 020 & 01 & 246 & & 4.57E-07 TY & 12 & 08 & 0.000088 LB & E6FT3S \\
\hline 020 & 01 & 253 & 253 & 1.25E-07 TY & 12 & 08 & 0.000024 LB & E6FT3S \\
\hline 020 & 01 & 91203 & & 3.17E-06 TY & 12 & 08 & 0.00061 LB & E6FT3S \\
\hline 020 & 01 & 93 & & 1.04E-06 TY & 12 & 08 & 0.0002 LB & E6FT3S \\
\hline 020 & 01 & $B Z$ & 71432 & 1.09E-05 TY & 12 & 08 & 0.0021 LB & E6FT3S \\
\hline 020 & 01 & $\mathrm{CO}$ & 630080 & 4.36E-01 TY & 12 & 08 & 84 LB & E6FT3S \\
\hline 020 & 01 & FORM & 50000 & 3.90E-04 TY & 12 & 08 & 0.075 LB & E6FT3S \\
\hline 020 & 01 & $\mathrm{NH} 3$ & 7664417 & TY & 12 & 00 & & \\
\hline 020 & 01 & NO2 & 10102440 & 5.20E-01 TY & 12 & 08 & 100 LB & E6FT3S \\
\hline 020 & 01 & PM10 & & 3.95E-02 TY & 12 & 08 & $7.6 \mathrm{LB}$ & E6FT3S \\
\hline 020 & 01 & PM25 & & 3.95E-02 TY & 12 & 08 & $7.6 \mathrm{LB}$ & E6FT3S \\
\hline 020 & 01 & PT & & 3.95E-02 TY & 12 & 08 & $7.6 \mathrm{LB}$ & E6FT3S \\
\hline 020 & 01 & $\mathrm{SO} 2$ & 7446095 & 3.12E-03 TY & 12 & 08 & 0.6 LB & E6FT3S \\
\hline 020 & 01 & TOLU & 108883 & 1.77E-05 TY & 12 & 08 & 0.0034 LB & E6FT3S \\
\hline 020 & 01 & VOC & & $2.86 \mathrm{E}-02 \mathrm{TY}$ & 12 & 08 & 5.5 LB & E6FT3S \\
\hline 021 & 01 & 106467 & & $6.23 \mathrm{E}-06 \mathrm{TY}$ & 12 & 08 & 0.0012 LB & E6FT3S \\
\hline 021 & 01 & 109 & & $6.23 \mathrm{E}-08 \mathrm{TY}$ & 12 & 08 & 0.000012 LB & E6FT3S \\
\hline 021 & 01 & 110543 & 110543 & 9.35E-03 TY & 12 & 08 & 1.8 LB & E6FT3S \\
\hline 021 & 01 & 125 & & 5.72E-06 TY & 12 & 08 & 0.0011 LB & E6FT3S \\
\hline 021 & 01 & 136 & 136 & 7.27E-06 TY & 12 & 08 & 0.0014 LB & E6FT3S \\
\hline 021 & 01 & 139 & 139 & 4.36E-07 TY & 12 & 08 & 0.000084 LB & E6FT3S \\
\hline 021 & 01 & 195 & 195 & 2.60E-06 TY & 12 & 08 & 0.0005 LB & E6FT3S \\
\hline 021 & 01 & 198 & 198 & 1.97E-06 TY & 12 & 08 & 0.00038 LB & E6FT3S \\
\hline 021 & 01 & 199 & 199 & 1.35E-06 TY & 12 & 08 & 0.00026 LB & E6FT3S \\
\hline 021 & 01 & 226 & 226 & 1.09E-05 TY & 12 & 08 & 0.0021 LB & E6FT3S \\
\hline 021 & 01 & 246 & & 4.57E-07 TY & 12 & 08 & 0.000088 LB & E6FT3S \\
\hline 021 & 01 & 253 & 253 & 1.25E-07 TY & 12 & 08 & 0.000024 LB & E6FT3S \\
\hline 021 & 01 & 91203 & & 3.17E-06 TY & 12 & 08 & 0.00061 LB & E6FT3S \\
\hline 021 & 01 & 93 & & 1.04E-06 TY & 12 & 08 & 0.0002 LB & E6FT3S \\
\hline 021 & 01 & $B Z$ & 71432 & 1.09E-05 TY & 12 & 08 & 0.0021 LB & E6FT3S \\
\hline 021 & 01 & $\mathrm{CO}$ & 630080 & 4.36E-01 TY & 12 & 08 & 84 LB & E6FT3S \\
\hline 021 & 01 & FORM & 50000 & $3.90 \mathrm{E}-04 \mathrm{TY}$ & 12 & 08 & 0.075 LB & E6FT3S \\
\hline 021 & 01 & $\mathrm{NH} 3$ & 7664417 & TY & 12 & 00 & & \\
\hline 021 & 01 & NO2 & 10102440 & 5.20E-01 TY & 12 & 08 & 100 LB & E6FT3S \\
\hline 021 & 01 & PM10 & & 3.95E-02 TY & 12 & 08 & $7.6 \mathrm{LB}$ & E6FT3S \\
\hline 021 & 01 & PM25 & & 3.95E-02 TY & 12 & 08 & $7.6 \mathrm{LB}$ & E6FT3S \\
\hline 021 & 01 & PT & & 3.95E-02 TY & 12 & 08 & $7.6 \mathrm{LB}$ & E6FT3S \\
\hline 021 & 01 & $\mathrm{SO} 2$ & 7446095 & 3.12E-03 TY & 12 & 08 & 0.6 LB & E6FT3S \\
\hline 021 & 01 & TOLU & 108883 & 1.77E-05 TY & 12 & 08 & 0.0034 LB & E6FT3S \\
\hline 021 & 01 & VOC & & 2.86E-02 TY & 12 & 08 & $5.5 \mathrm{LB}$ & E6FT3S \\
\hline 022 & 01 & $\mathrm{CO}$ & 630080 & $0 T Y$ & 12 & 08 & & \\
\hline 022 & 01 & $\mathrm{NH} 3$ & 7664417 & OTY & 12 & 08 & & \\
\hline 022 & 01 & NO2 & 10102440 & 0 TY & 12 & 08 & & \\
\hline 022 & 01 & PM10 & & OTY & 12 & 08 & & \\
\hline 022 & 01 & PM25 & Which ou & OTY & 12 & 08 & & \\
\hline 022 & 01 & PT & $3-25 x=8 x=$ & OTY & 12 & 08 & & \\
\hline
\end{tabular}




\begin{tabular}{|c|c|c|c|c|c|c|c|c|}
\hline 022 & 01 & $\mathrm{SO} 2$ & 7446095 & $0 T Y$ & 12 & 08 & & \\
\hline 022 & 01 & VOC & & 0 TY & 12 & 08 & & \\
\hline 023 & 01 & $\mathrm{CO}$ & 630080 & $0 T Y$ & 12 & 08 & & \\
\hline 023 & 01 & $\mathrm{NH} 3$ & 7664417 & 0 TY & 12 & 08 & & \\
\hline 023 & 01 & $\mathrm{NO} 2$ & 10102440 & 0 TY & 12 & 08 & & \\
\hline 023 & 01 & PM10 & & $0 T Y$ & 12 & 08 & & \\
\hline 023 & 01 & PM25 & & 0 TY & 12 & 08 & & \\
\hline 023 & 01 & PT & & OTY & 12 & 08 & & \\
\hline 023 & 01 & $\mathrm{SO} 2$ & 7446095 & OTY & 12 & 08 & & \\
\hline 023 & 01 & VOC & & OTY & 12 & 08 & & \\
\hline 024 & 01 & 106467 & & $1.48 \mathrm{E}-05 \mathrm{TY}$ & 12 & 08 & 0.0012 LB & E6FT3S \\
\hline 024 & 01 & 109 & 630080 & 1.48E-07 TY & 12 & 08 & 0.000012 LB & E6FT3S \\
\hline 024 & 01 & 110543 & 110543 & 2.23E-02 TY & 12 & 08 & $1.8 \mathrm{LB}$ & E6FT3S \\
\hline 024 & 01 & 125 & 630080 & 1.36E-05 TY & 12 & 08 & 0.0011 LB & E6FT3S \\
\hline 024 & 01 & 136 & 136 & 1.73E-05 TY & 12 & 08 & 0.0014 LB & E6FT3S \\
\hline 024 & 01 & 139 & 139 & 1.04E-06 TY & 12 & 08 & 0.000084 LB & E6FT3S \\
\hline 024 & 01 & 195 & 195 & 6.18E-06 TY & 12 & 08 & 0.0005 LB & E6FT3S \\
\hline 024 & 01 & 198 & 198 & 4.70E-06 TY & 12 & 08 & 0.00038 LB & E6FT3S \\
\hline 024 & 01 & 199 & 199 & $3.22 \mathrm{E}-06 \mathrm{TY}$ & 12 & 08 & 0.00026 LB & E6FT3S \\
\hline 024 & 01 & 226 & 226 & 2.60E-05 TY & 12 & 08 & 0.0021 LB & E6FT3S \\
\hline 024 & 01 & 246 & 630080 & 1.09E-06 TY & 12 & 08 & 0.000088 LB & E6FT3S \\
\hline 024 & 01 & 253 & 253 & 2.97E-07 TY & 12 & 08 & 0.000024 LB & E6FT3S \\
\hline 024 & 01 & 91203 & 630080 & 7.54E-06 TY & 12 & 08 & 0.00061 LB & E6FT3S \\
\hline 024 & 01 & 93 & & 2.47E-06 TY & 12 & 08 & 0.0002 LB & E6FT3S \\
\hline 024 & 01 & $B Z$ & 71432 & 2.60E-05 TY & 12 & 08 & 0.0021 LB & E6FT3S \\
\hline 024 & 01 & $\mathrm{CO}$ & 630080 & 4.59E-01 TY & 12 & 07 & 37.08 LB & E6FT3S \\
\hline 024 & 01 & FORM & 50000 & 9.28E-04 TY & 12 & 08 & 0.075 LB & E6FT3S \\
\hline 024 & 01 & $\mathrm{NH} 3$ & 7664417 & TY & 12 & 00 & & \\
\hline 024 & 01 & $\mathrm{NO} 2$ & 10102440 & 4.59E-01 TY & 12 & 07 & 37.08 LB & E6FT3S \\
\hline 024 & 01 & PM10 & & $9.40 E-02 T Y$ & 12 & 08 & $7.6 \mathrm{LB}$ & E6FT3S \\
\hline 024 & 01 & PM25 & & $9.40 \mathrm{E}-02 \mathrm{TY}$ & 12 & 08 & $7.6 \mathrm{LB}$ & E6FT3S \\
\hline 024 & 01 & PT & & $9.40 E-02$ TY & 12 & 08 & $7.6 \mathrm{LB}$ & E6FT3S \\
\hline 024 & 01 & $\mathrm{SO} 2$ & 7446095 & 7.42E-03 TY & 12 & 08 & 0.6 LB & E6FT3S \\
\hline 024 & 01 & TOLU & 108883 & 4.20E-05 TY & 12 & 08 & 0.0034 LB & E6FT3S \\
\hline 024 & 01 & VOC & & 6.80E-02 TY & 12 & 08 & 5.5 LB & E6FT3S \\
\hline
\end{tabular}




\begin{tabular}{|c|c|c|c|c|c|c|}
\hline 030 & 01 & 3 CLET & 79016 & OTY & 12 & 03 \\
\hline 030 & 01 & $\mathrm{NH} 3$ & 7664417 & OTY & 12 & 03 \\
\hline 030 & 01 & PM10 & & OTY & 12 & 03 \\
\hline 030 & 01 & PM25 & & 0 TY & 12 & 03 \\
\hline 031 & 01 & 100027 & 100027 & $2.50 \mathrm{E}-05 \mathrm{TY}$ & 12 & 03 \\
\hline 031 & 01 & 100414 & 100414 & $0 T Y$ & 12 & 03 \\
\hline 031 & 01 & 100425 & 100425 & 2.25E-03 TY & 12 & 03 \\
\hline 031 & 01 & 100447 & 100447 & 1.20E-04 TY & 12 & 03 \\
\hline 031 & 01 & 101688 & 101688 & OTY & 12 & 03 \\
\hline 031 & 01 & 106423 & & 2.08E-03 TY & 12 & 03 \\
\hline 031 & 01 & 106445 & & 1.10E-04 TY & 12 & 03 \\
\hline 031 & 01 & 106503 & 106503 & $5.50 \mathrm{E}-04 \mathrm{TY}$ & 12 & 03 \\
\hline 031 & 01 & 106514 & & 0 TY & 12 & 03 \\
\hline 031 & 01 & 106887 & 106887 & 1.75E-04 TY & 12 & 03 \\
\hline 031 & 01 & 106898 & 106898 & OTY & 12 & 03 \\
\hline 031 & 01 & 106934 & 106934 & 1.10E-04 TY & 12 & 03 \\
\hline 031 & 01 & 106990 & 106990 & OTY & 12 & 03 \\
\hline 031 & 01 & 107028 & 107028 & OTY & 12 & 03 \\
\hline 031 & 01 & 107062 & 107062 & 4.77E-03 TY & 12 & 03 \\
\hline 031 & 01 & 107131 & 107131 & OTY & 12 & 03 \\
\hline 031 & 01 & 107211 & 107211 & 2.56E-01 TY & 12 & 03 \\
\hline 031 & 01 & 107302 & & 0 TY & 12 & 03 \\
\hline 031 & 01 & 108054 & 108054 & OTY & 12 & 03 \\
\hline 031 & 01 & 108101 & 108101 & OTY & 12 & 03 \\
\hline 031 & 01 & 108383 & & 2.58E-03 TY & 12 & 03 \\
\hline 031 & 01 & 108394 & 108394 & 1.10E-04 TY & 12 & 03 \\
\hline 031 & 01 & 108907 & 108907 & 1.21E-02 TY & 12 & 03 \\
\hline 031 & 01 & 108952 & 108952 & 1.91E-03 TY & 12 & 03 \\
\hline 031 & 01 & 109 & & $3.15 \mathrm{E}-04 \mathrm{TV}$ & 12 & 03 \\
\hline 031 & 01 & 110543 & 110543 & $2.31 \mathrm{E}-01 \mathrm{TY}$ & 12 & 03 \\
\hline 031 & 01 & 111422 & 111422 & 6.00E-04 TY & 12 & 03 \\
\hline 031 & 01 & 1120714 & 1120714 & OTY & 12 & 03 \\
\hline 031 & 01 & 117817 & 117817 & 5.00E-06 TY & 12 & 03 \\
\hline 031 & 01 & 120809 & 120809 & 5.50E-04 TY & 12 & 03 \\
\hline 031 & 01 & 120821 & 120821 & OTY & 12 & 03 \\
\hline 031 & 01 & 121448 & 121448 & 5.37E-03 TY & 12 & 03 \\
\hline 031 & 01 & 121697 & & OTY & 12 & 03 \\
\hline 031 & 01 & 122667 & & OTY & 12 & 03 \\
\hline 031 & 01 & 123319 & 123319 & 1.05E-04 TY & 12 & 03 \\
\hline 031 & 01 & 123386 & 123386 & OTY & 12 & 03 \\
\hline 031 & 01 & 123911 & 123911 & 3.43E-03 TY & 12 & 03 \\
\hline 031 & 01 & 125 & & 6.40E-04 TY & 12 & 03 \\
\hline 031 & 01 & 127184 & 127184 & OTY & 12 & 03 \\
\hline 031 & 01 & 136 & 136 & 3.72E-03 TY & 12 & 03 \\
\hline 031 & 01 & 139 & 139 & 2.66E-03 TY & 12 & 03 \\
\hline 031 & 01 & 140885 & 140885 & OTY & 12 & 03 \\
\hline 031 & 01 & 144 & & 5.65E-02 TY & 12 & 03 \\
\hline 031 & 01 & 156627 & & OTY & 12 & 03 \\
\hline 031 & 01 & 1634044 & 1634044 & 1.03E-02 TY & 12 & 03 \\
\hline 031 & 01 & 171 & & 4.50E-02 TY & 12 & 03 \\
\hline 031 & 01 & 195 & 195 & 1.63E-03 TY & 12 & 03 \\
\hline 031 & 01 & 198 & 198 & 5.15E-01 TY & 12 & 03 \\
\hline 031 & 01 & 199 & 199 & 9.70E-04 TY & 12 & 03 \\
\hline 031 & 01 & 226 & 226 & 5.37E-03 TY & 12 & 03 \\
\hline 031 & 01 & 234 & & OTY & 12 & 03 \\
\hline 031 & 01 & 246 & & 4.80E-03 TY & 12 & 03 \\
\hline 031 & 01 & 253 & 253 & 1.01E-02 TY & 12 & 03 \\
\hline 031 & 01 & 302012 & 302012 & 1.10E-03 TY & 12 & 03 \\
\hline 031 & & 383 & & 1.65E-03 TY & 12 & 03 \\
\hline 031 & 01 & 3CLET & 79016 & oTY & 12 & 03 \\
\hline 031 & 01 & 463581 & 463581 & OTY & 12 & 03 \\
\hline 031 & 01 & 51285 & & 0 TY & 12 & 03 \\
\hline 031 & & 51796 & 51796 & 7.35E-04 TY & 12 & 03 \\
\hline 031 & 01 & 540841 & 540841 & 4.58E-03 TY & 12 & 03 \\
\hline 031 & 01 & 542881 & 542881 & $0 T Y$ & 12 & 03 \\
\hline 031 & 01 & 56235 & 56235 & 8.79E-03 TY & 12 & 03 \\
\hline 031 & 01 & 57125 & 57125 & OTY & 12 & 03 \\
\hline 031 & 01 & 57147 & 57147 & 0 TY & 12 & 03 \\
\hline 031 & 01 & 584849 & 584849 & OTY & 12 & 03 \\
\hline 031 & 01 & 60344 & & 0 TY & 12 & 03 \\
\hline 031 & 01 & 62533 & 62533 & $6.45 \mathrm{E}-04 \mathrm{TY}$ & 12 & 03 \\
\hline 031 & 01 & 67561 & 67561 & 7.29E-01 TY & 12 & 03 \\
\hline 031 & 01 & 67663 & 67663 & 1.25E-01 TY & 12 & 03 \\
\hline 031 & 01 & 680319 & 680319 & $0 T Y$ & 12 & 03 \\
\hline 031 & 01 & 68122 & 68122 & 1.40E-01 TY & 12 & 03 \\
\hline 031 & 01 & 71556 & 71556 & OTY & 12 & 03 \\
\hline 031 & 01 & 7439921 & 7439921 & $0 T Y$ & 12 & 03 \\
\hline 031 & 01 & 7439976 & 7439976 & $0 T Y$ & 12 & 03 \\
\hline 031 & 01 & 7440020 & 7440020 & OTY & 12 & 03 \\
\hline 031 & 01 & 7440360 & 7440360 & OTY & 12 & 03 \\
\hline
\end{tabular}




\begin{tabular}{|c|c|c|c|c|c|c|c|c|}
\hline 031 & 01 & 7440382 & 7440382 & OTY & 12 & 03 & & \\
\hline 031 & 01 & 7440417 & 7440417 & OTY & 12 & 03 & & \\
\hline 031 & 01 & 7440439 & 7440439 & OTY & 12 & 03 & & \\
\hline 031 & 01 & 7440473 & 7440473 & OTY & 12 & 03 & & \\
\hline 031 & 01 & 7440484 & 7440484 & OTY & 12 & 03 & & \\
\hline 031 & 01 & 74839 & 74839 & OTY & 12 & 03 & & \\
\hline 031 & 01 & 74873 & 74873 & OTY & 12 & 03 & & \\
\hline 031 & 01 & 74884 & 74884 & OTY & 12 & 03 & & \\
\hline 031 & 01 & 75003 & 75003 & 0TY & 12 & 03 & & \\
\hline 031 & 01 & 75058 & 75058 & 7.03E-01 TY & 12 & 03 & & \\
\hline 031 & 01 & 75070 & 75070 & 0TY & 12 & 03 & & \\
\hline 031 & 01 & 75092 & 75092 & 6.32E-01 TY & 12 & 03 & & \\
\hline 031 & 01 & 75150 & 75150 & 3.00E-05 TY & 12 & 03 & & \\
\hline 031 & 01 & 75252 & & 7.50E-05 TY & 12 & 03 & & \\
\hline 031 & 01 & 75343 & 75343 & 0 TY & 12 & 03 & & \\
\hline 031 & 01 & 75354 & 75354 & 0TY & 12 & 03 & & \\
\hline 031 & 01 & 7550450 & 7550450 & 4.75E-04 TY & 12 & 03 & & \\
\hline 031 & 01 & 75558 & & 0 TY & 12 & 03 & & \\
\hline 031 & 01 & 75569 & 75569 & $3.50 \mathrm{E}-05 \mathrm{TY}$ & 12 & 03 & & \\
\hline 031 & 01 & 7664393 & 7664393 & $2.09 \mathrm{E}-02 \mathrm{TY}$ & 12 & 03 & & \\
\hline 031 & 01 & 7723140 & 7723140 & $5.00 \mathrm{E}-04 \mathrm{TY}$ & 12 & 03 & & \\
\hline 031 & 01 & 77474 & 77474 & 0 TY & 12 & 03 & & \\
\hline 031 & 01 & 77781 & 77781 & $1.00 \mathrm{E}-05 \mathrm{TY}$ & 12 & 03 & & \\
\hline 031 & 01 & 7782492 & 7782492 & 0TY & 12 & 03 & & \\
\hline 031 & 01 & 7803512 & 7803512 & 0 TY & 12 & 03 & & \\
\hline 031 & 01 & 79005 & 79005 & OTY & 12 & 03 & & \\
\hline 031 & 01 & 79061 & 79061 & 5.45E-03 TY & 12 & 03 & & \\
\hline 031 & 01 & 79107 & 79107 & OTY & 12 & 03 & & \\
\hline 031 & 01 & 79118 & 79118 & OTY & 12 & 03 & & \\
\hline 031 & 01 & 79345 & 79345 & 0 TY & 12 & 03 & & \\
\hline 031 & 01 & 80626 & 80626 & 3.17E-03 TY & 12 & 03 & & \\
\hline 031 & 01 & 822060 & & $2.50 \mathrm{E}-05 \mathrm{TY}$ & 12 & 03 & & \\
\hline 031 & 01 & 84742 & 84742 & 3.31E-03 TY & 12 & 03 & & \\
\hline 031 & 01 & 85449 & 85449 & OTY & 12 & 03 & & \\
\hline 031 & 01 & 91203 & 91203 & 2.80E-04 TY & 12 & 03 & & \\
\hline 031 & & 91225 & 91225 & 1.15E-04 TY & 12 & 03 & & \\
\hline 031 & & 92 & & $6.60 \mathrm{E}-04 \mathrm{TY}$ & 12 & 03 & & \\
\hline 031 & 01 & 92671 & 92671 & 0TY & 12 & 03 & & \\
\hline 031 & 01 & 93 & & 1.50E-03 TY & 12 & 03 & & \\
\hline 031 & 01 & 95476 & 95476 & $5.82 \mathrm{E}-03 \mathrm{TY}$ & 12 & 03 & & \\
\hline 031 & 01 & 98828 & & OTY & 12 & 03 & & \\
\hline 031 & 01 & 98862 & 98862 & 5.60E-04 TY & 12 & 03 & & \\
\hline 031 & 01 & 98953 & 98953 & 1.32E-03 TY & 12 & 03 & & \\
\hline 031 & 01 & $\mathrm{BZ}$ & 71432 & 1.11E-02 TY & 12 & 03 & & \\
\hline 031 & 01 & $\mathrm{CL}$ & 7782505 & 1.28E-02 TY & 12 & 03 & & \\
\hline 031 & 01 & FORM & 50000 & $6.58 \mathrm{E}-02 \mathrm{TY}$ & 12 & 03 & & \\
\hline 031 & 01 & $\mathrm{HC} 36$ & 78933 & 3.64E-01 TY & 12 & 03 & & \\
\hline 031 & 01 & $\mathrm{HC} 81$ & 1330207 & 4.16E-03 TY & 12 & 03 & & \\
\hline 031 & 01 & $\mathrm{HCL}$ & 7647010 & $1.52 \mathrm{E}+00 \mathrm{TY}$ & 12 & 03 & & \\
\hline 031 & 01 & MN-PT & 7439965 & OTY & 12 & 03 & & \\
\hline 031 & 01 & $\mathrm{NH} 3$ & 7664417 & 5.91E-02 TY & 12 & 03 & & \\
\hline 031 & 01 & PM10 & & OTY & 12 & 03 & & \\
\hline 031 & 01 & PM25 & & OTY & 12 & 03 & & \\
\hline 031 & 01 & TOLU & 108883 & 1.42E-01 TY & 12 & 03 & & \\
\hline 031 & 01 & VOC & & $7.95 \mathrm{E}+00 \mathrm{TY}$ & 12 & 03 & & \\
\hline 032 & 01 & 106467 & & 1.52E-04 TY & 12 & 08 & 0.0012 LB & E6FT3S \\
\hline 032 & 01 & 109 & & $6.41 E-06$ TY & 12 & 08 & 0.000012 LB & E6FT3S \\
\hline 032 & 01 & 110543 & 110543 & 2.27E-01 TY & 12 & 08 & 1.8 LB & E6FT3S \\
\hline 032 & 01 & 125 & & 1.44E-04 TY & 12 & 08 & 0.0011 LB & E6FT3S \\
\hline 032 & 01 & 136 & 136 & 1.82E-04 TY & 12 & 08 & 0.0014 LB & E6FT3S \\
\hline 032 & 01 & 139 & 139 & 1.06E-05 TY & 12 & 08 & 0.000084 LB & E6FT3S \\
\hline 032 & 01 & 195 & 195 & 7.78E-05 TY & 12 & 08 & 0.0005 LB & E6FT3S \\
\hline 032 & 01 & 198 & 198 & 5.78E-05 TY & 12 & 08 & 0.00038 LB & E6FT3S \\
\hline 032 & 01 & 199 & 199 & 3.77E-05 TY & 12 & 08 & 0.00026 LB & E6FT3S \\
\hline 032 & 01 & 226 & 226 & $2.70 \mathrm{E}-04 \mathrm{TY}$ & 12 & 08 & 0.0021 LB & E6FT3S \\
\hline 032 & 01 & 246 & & 5.04E-05 TY & 12 & 08 & 0.000088 LB & E6FT3S \\
\hline 032 & 01 & 253 & 253 & $2.75 E-05 T Y$ & 12 & 08 & 0.000024 LB & E6FT3S \\
\hline 032 & 01 & 91203 & 7446095 & 7.71E-05 TY & 12 & 08 & 0.00061 LB & E6FT3S \\
\hline 032 & 01 & 93 & & 3.18E-05 TY & 12 & 08 & 0.0002 LB & E6FT3S \\
\hline 032 & 01 & $B Z$ & 71432 & 2.65E-04 TY & 12 & 08 & 0.0021 LB & E6FT3S \\
\hline 032 & 01 & $\mathrm{CO}$ & 630080 & $5.11 \mathrm{E}+00 \mathrm{TY}$ & 12 & 08 & 40 LB & E6FT3S \\
\hline 032 & 01 & FORM & 50000 & 1.00E-02 TY & 12 & 08 & 0.075 LB & E6FT3S \\
\hline 032 & 01 & $\mathrm{NH} 3$ & 7664417 & TY & 12 & 00 & & \\
\hline 032 & 01 & NO2 & 10102440 & $7.43 E+00 \mathrm{TY}$ & 12 & 04 & 58 LB & E6FT3S \\
\hline 032 & 01 & PM10 & & 9.90E-01 TY & 12 & 08 & $7.6 \mathrm{LB}$ & E6FT3S \\
\hline 032 & 01 & PM25 & & 9.80E-01 TY & 12 & 08 & $7.6 \mathrm{LB}$ & E6FT3S \\
\hline 032 & 01 & PT & & 1.00E+00 TY & 12 & 08 & $7.6 \mathrm{LB}$ & E6FT3S \\
\hline 032 & 01 & $\mathrm{SO} 2$ & 7446095 & 1.60E-01 TY & 12 & 08 & 0.6 LB & E6FT3S \\
\hline 032 & 01 & TOLU & 108883 & 4.29E-04 TY & 12 & 08 & 0.0034 LB & E6FT3S \\
\hline 032 & 01 & VOC & 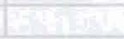 & 7.00E-01 TY & 12 & 08 & 5.5 LB & E6FT3S \\
\hline
\end{tabular}




\begin{tabular}{|c|c|c|c|c|c|c|c|c|}
\hline 033 & 01 & 106467 & 10102440 & 2.59E-05 TY & 12 & 08 & 0.0012 LB & E6FT3S \\
\hline 033 & 01 & 109 & & 3.03E-07 TY & 12 & 08 & 0.000012 LB & E6FT3S \\
\hline 033 & 01 & 110543 & 110543 & 3.88E-02 TY & 12 & 08 & $1.8 \mathrm{LB}$ & E6FT3S \\
\hline 033 & 01 & 125 & 630080 & 2.38E-05 TY & 12 & 08 & 0.0011 LB & E6FT3S \\
\hline 033 & 01 & 136 & 136 & 3.02E-05 TY & 12 & 08 & 0.0014 LB & E6FT3S \\
\hline 033 & 01 & 139 & 139 & 1.81E-06 TY & 12 & 08 & 0.000084 LB & E6FT3S \\
\hline 033 & 01 & 195 & 195 & 1.09E-05 TY & 12 & 08 & 0.0005 LB & E6FT3S \\
\hline 033 & 01 & 198 & 198 & 8.28E-06 TY & 12 & 08 & 0.00038 LB & E6FT3S \\
\hline 033 & 01 & 199 & 199 & 5.65E-06 TY & 12 & 08 & 0.00026 LB & E6FT3S \\
\hline 033 & 01 & 226 & 226 & 4.53E-05 TY & 12 & 08 & 0.0021 LB & E6FT3S \\
\hline 033 & 01 & 246 & 7446095 & 2.25E-06 TY & 12 & 08 & 0.000088 LB & E6FT3S \\
\hline 033 & 01 & 253 & 253 & 7.36E-07 TY & 12 & 08 & 0.000024 LB & E6FT3S \\
\hline 033 & 01 & 91203 & 7446095 & 1.32E-05 TY & 12 & 08 & 0.00061 LB & E6FT3S \\
\hline 033 & 01 & 93 & & 4.37E-06 TY & 12 & 08 & 0.0002 LB & E6FT3S \\
\hline 033 & 01 & $B Z$ & 71432 & 4.53E-05 TY & 12 & 08 & 0.0021 LB & E6FT3S \\
\hline 033 & 01 & $\mathrm{CO}$ & 630080 & 8.60E-01 TY & 12 & 08 & 40 LB & E6FT3S \\
\hline 033 & 01 & FORM & 50000 & 1.62E-03 TY & 12 & 08 & 0.075 LB & E6FT3S \\
\hline 033 & 01 & $\mathrm{NH} 3$ & 7664417 & TY & 12 & 00 & & \\
\hline 033 & 01 & $\mathrm{NO} 2$ & 10102440 & $1.25 \mathrm{E}+00 \mathrm{TY}$ & 12 & 04 & 58 LB & E6FT3S \\
\hline 033 & 01 & PM10 & & 1.60E-01 TY & 12 & 08 & $7.6 \mathrm{LB}$ & E6FT3S \\
\hline 033 & 01 & PM25 & & 1.60E-01 TY & 12 & 08 & $7.6 \mathrm{LB}$ & E6FT3S \\
\hline 033 & 01 & PT & & 1.60E-01 TY & 12 & 08 & 7.6 LB & E6FT3S \\
\hline 033 & 01 & $\mathrm{SO} 2$ & 7446095 & 1.00E-02 TY & 12 & 08 & 0.6 LB & E6FT3S \\
\hline 033 & 01 & TOLU & 108883 & 7.33E-05 TY & 12 & 08 & 0.0034 LB & E6FT3S \\
\hline 033 & 01 & VOC & & 1.20E-01 TY & 12 & 08 & 5.5 LB & E6FT3S \\
\hline 034 & 01 & 106467 & 10102440 & $1.58 \mathrm{E}-04 \mathrm{TY}$ & 12 & 08 & 0.0012 LB & E6FT3S \\
\hline 034 & 01 & 109 & 630080 & 3.62E-06 TY & 12 & 08 & 0.000012 LB & E6FT3S \\
\hline 034 & 01 & 110543 & 110543 & 2.36E-01 TY & 12 & 08 & $1.8 \mathrm{LB}$ & E6FT3S \\
\hline 034 & 01 & 125 & 7446095 & 1.47E-04 TY & 12 & 08 & 0.0011 LB & E6FT3S \\
\hline 034 & 01 & 136 & 136 & 1.86E-04 TY & 12 & 08 & 0.0014 LB & E6FT3S \\
\hline 034 & 01 & 139 & 139 & $1.10 \mathrm{E}-05 \mathrm{TY}$ & 12 & 08 & 0.000084 LB & E6FT3S \\
\hline 034 & 01 & 195 & 195 & 7.18E-05 TY & 12 & 08 & 0.0005 LB & E6FT3S \\
\hline 034 & 01 & 198 & 198 & $5.40 \mathrm{E}-05 \mathrm{TY}$ & 12 & 08 & 0.00038 LB & E6FT3S \\
\hline 034 & 01 & 199 & 199 & 3.62E-05 TY & 12 & 08 & 0.00026 LB & E6FT3S \\
\hline 034 & 01 & 226 & 226 & $2.78 \mathrm{E}-04 \mathrm{TY}$ & 12 & 08 & 0.0021 LB & E6FT3S \\
\hline 034 & 01 & 246 & 7446095 & $2.80 \mathrm{E}-05 \mathrm{TY}$ & 12 & 08 & 0.000088 LB & E6FT3S \\
\hline 034 & 01 & 253 & 253 & $1.34 \mathrm{E}-05 \mathrm{TY}$ & 12 & 08 & 0.000024 LB & E6FT3S \\
\hline 034 & 01 & 91203 & 630080 & 8.01E-05 TY & 12 & 08 & 0.00061 LB & E6FT3S \\
\hline 034 & 01 & 93 & 630080 & $2.90 \mathrm{E}-05 \mathrm{TY}$ & 12 & 08 & 0.0002 LB & E6FT3S \\
\hline 034 & 01 & $B Z$ & 71432 & 2.76E-04 TY & 12 & 08 & 0.0021 LB & E6FT3S \\
\hline 034 & 01 & $\mathrm{CO}$ & 630080 & $5.28 \mathrm{E}+00 \mathrm{TY}$ & 12 & 08 & 40 LB & E6FT3S \\
\hline 034 & 01 & FORM & 50000 & 1.01E-02 TY & 12 & 08 & 0.075 LB & E6FT3S \\
\hline 034 & 01 & $\mathrm{NH} 3$ & 7664417 & TY & 12 & 00 & & \\
\hline 034 & 01 & NO2 & 10102440 & $7.66 \mathrm{E}+00 \mathrm{Tr}$ & 12 & 04 & 58 LB & E6FT3S \\
\hline 034 & 01 & PM10 & & $1.01 E+00 T Y$ & 12 & 08 & 7.6 LB & E6FT3S \\
\hline 034 & 01 & PM25 & & $1.01 E+00 \mathrm{TY}$ & 12 & 08 & $7.6 \mathrm{LB}$ & E6FT3S \\
\hline 034 & 01 & PT & & $1.02 E+00 \mathrm{TY}$ & 12 & 08 & 7.6 LB & E6FT3S \\
\hline 034 & 01 & $\mathrm{SO} 2$ & 7446095 & 1.20E-01 TY & 12 & 08 & 0.6 LB & E6FT3S \\
\hline 034 & 01 & TOLU & 108883 & 4.47E-04 TY & 12 & 08 & 0.0034 LB & E6FT3S \\
\hline 034 & 01 & VOC & & 7.20E-01 TY & 12 & 08 & 5.5 LB & E6FT3S \\
\hline 035 & 01 & VOC & & 6.94E-03 TY & 12 & 08 & & \\
\hline 036 & 01 & VOC & & 3.30E-02 TY & 12 & 08 & & \\
\hline 037 & 01 & 106467 & & 3.79E-06 TY & 12 & 08 & 0.0012 LB & E6FT3S \\
\hline 037 & 01 & 109 & & $3.79 \mathrm{E}-08 \mathrm{TY}$ & 12 & 08 & 0.000012 LB & E6FT3S \\
\hline 037 & 01 & 110543 & 110543 & $5.68 \mathrm{E}-03 \mathrm{TY}$ & 12 & 08 & $1.8 \mathrm{LB}$ & E6FT3S \\
\hline 037 & 01 & 125 & 7446095 & 3.47E-06 TY & 12 & 08 & 0.0011 LB & E6FT3S \\
\hline 037 & 01 & 136 & 136 & 4.42E-06 TY & 12 & 08 & 0.0014 LB & E6FT3S \\
\hline 037 & 01 & 139 & 139 & 2.65E-07 TY & 12 & 08 & 0.000084 LB & E6FT3S \\
\hline 037 & 01 & 195 & 195 & $1.58 \mathrm{E}-06 \mathrm{TY}$ & 12 & 08 & 0.0005 LB & E6FT3S \\
\hline 037 & 01 & 198 & 198 & 1.20E-06 TY & 12 & 08 & 0.00038 LB & E6FT3S \\
\hline 037 & 01 & 199 & 199 & 8.21E-07 TY & 12 & 08 & 0.00026 LB & E6FT3S \\
\hline 037 & 01 & 226 & 226 & 6.63E-06 TY & 12 & 08 & 0.0021 LB & E6FT3S \\
\hline 037 & 01 & 246 & 7446095 & $2.78 \mathrm{E}-07 \mathrm{TY}$ & 12 & 08 & 0.000088 LB & E6FT3S \\
\hline 037 & 01 & 253 & 253 & 7.58E-08 TY & 12 & 08 & 0.000024 LB & E6FT3S \\
\hline 037 & 01 & 91203 & 7446095 & 1.93E-06 TY & 12 & 08 & 0.00061 LB & E6FT3S \\
\hline 037 & 01 & 93 & 7446095 & 6.31E-07 TY & 12 & 08 & 0.0002 LB & E6FT3S \\
\hline 037 & 01 & $B Z$ & 71432 & 6.63E-06 TY & 12 & 08 & 0.0021 LB & E6FT3S \\
\hline 037 & 01 & $\mathrm{CO}$ & 630080 & 1.21E-01 TY & 12 & 07 & 38.2 LB & E6FT3S \\
\hline 037 & 01 & FORM & 50000 & 2.37E-04 TY & 12 & 08 & 0.075 LB & E6FT3S \\
\hline 037 & 01 & $\mathrm{NH} 3$ & 7664417 & TY & 12 & 00 & & \\
\hline 037 & 01 & NO2 & 10102440 & 4.36E-01 TY & 12 & 04 & 138 LB & E6FT3S \\
\hline 037 & 01 & PM10 & & 4.48E-02 TY & 12 & 07 & 14.2 LB & E6FT3S \\
\hline 037 & 01 & PM25 & & 4.48E-02 TY & 12 & 07 & 14.2 LB & E6FT3S \\
\hline 037 & 01 & PT & & 4.48E-02 TY & 12 & 07 & 14.2 LB & E6FT3S \\
\hline 037 & 01 & $\mathrm{SO} 2$ & 7446095 & 1.89E-03 TY & 12 & 08 & 0.6 LB & E6FT3S \\
\hline 037 & 01 & TOLU & 108883 & 1.07E-05 TY & 12 & 08 & 0.0034 LB & E6FT3S \\
\hline 037 & 01 & VOC & & 1.89E-02 TY & 12 & 07 & 5.98 LB & E6FT3S \\
\hline 038 & 01 & 106467 & 630080 & 1.29E=05 TY & 12 & 08 & 0.0012 LB & E6FT3S \\
\hline 038 & 01 & 109 & 630080 & 1.29E-07 TY & 12 & 08 & 0.000012 LB & E6FT3S \\
\hline 038 & 01 & 110543 & 110543 & 1.93E-02 TY & 12 & 08 & $1.8 \mathrm{LB}$ & E6FT3S \\
\hline
\end{tabular}




\begin{tabular}{|c|c|c|c|c|c|c|c|c|}
\hline 038 & 01 & 125 & & 1.18E-05 TY & 12 & 08 & 0.0011 LB & E6FT3S \\
\hline 038 & 01 & 136 & 136 & 1.50E-05 TY & 12 & 08 & 0.0014 LB & E6FT3S \\
\hline 038 & 01 & 139 & 139 & 9.01E-07 TY & 12 & 08 & 0.000084 LB & E6FT3S \\
\hline 038 & 01 & 195 & 195 & 5.37E-06 TY & 12 & 08 & 0.0005 LB & E6FT3S \\
\hline 038 & 01 & 198 & 198 & 4.08E-06 TY & 12 & 08 & 0.00038 LB & E6FT3S \\
\hline 038 & 01 & 199 & 199 & 2.79E-06 TY & 12 & 08 & 0.00026 LB & E6FT3S \\
\hline 038 & 01 & 226 & 226 & 2.25E-05 TY & 12 & 08 & 0.0021 LB & E6FT3S \\
\hline 038 & 01 & 246 & & 9.44E-07 TY & 12 & 08 & 0.000088 LB & E6FT3S \\
\hline 038 & 01 & 253 & 253 & $2.58 \mathrm{E}-07 \mathrm{TY}$ & 12 & 08 & 0.000024 LB & E6FT3S \\
\hline 038 & 01 & 91203 & & 6.55E-06 TY & 12 & 08 & 0.00061 LB & E6FT3S \\
\hline 038 & 01 & 93 & & 2.15E-06 TY & 12 & 08 & 0.0002 LB & E6FT3S \\
\hline 038 & 01 & $B Z$ & 71432 & 2.25E-05 TY & 12 & 08 & 0.0021 LB & E6FT3S \\
\hline 038 & 01 & $\mathrm{CO}$ & 630080 & 4.10E-01 TY & 12 & 07 & 38.2 LB & E6FT3S \\
\hline 038 & 01 & FORM & 50000 & 8.05E-04 TY & 12 & 08 & 0.075 LB & E6FT3S \\
\hline 038 & 01 & $\mathrm{NH} 3$ & 7664417 & TY & 12 & 00 & & \\
\hline 038 & 01 & NO2 & 10102440 & $1.48 \mathrm{E}+00 \mathrm{TY}$ & 12 & 04 & 138 LB & E6FT3S \\
\hline 038 & 01 & PM10 & & 1.52E-01 TY & 12 & 07 & 14.2 LB & E6FT3S \\
\hline 038 & 01 & PM25 & & 1.52E-01 TY & 12 & 07 & 14.2 LB & E6FT3S \\
\hline 038 & 01 & PT & & 1.52E-01 TY & 12 & 07 & 14.2 LB & E6FT3S \\
\hline 038 & 01 & $\mathrm{SO} 2$ & 7446095 & $6.44 \mathrm{E}-03 \mathrm{TY}$ & 12 & 08 & 0.6 LB & E6FT3S \\
\hline 038 & 01 & TOLU & 108883 & 3.65E-05 TY & 12 & 08 & 0.0034 LB & E6FT3S \\
\hline 038 & 01 & VOC & & $6.42 \mathrm{E}-02 \mathrm{TY}$ & 12 & 07 & 5.98 LB & E6FT3S \\
\hline 039 & 01 & 100027 & & OTY & 12 & 07 & & \\
\hline 039 & 01 & 100414 & 100414 & OTY & 12 & 07 & & \\
\hline 039 & 01 & 100425 & & OTY & 12 & 07 & & \\
\hline 039 & 01 & 106990 & & OTY & 12 & 07 & & \\
\hline 039 & 01 & 107028 & & OTY & 12 & 07 & & \\
\hline 039 & 01 & 107062 & & OTY & 12 & 07 & & \\
\hline 039 & 01 & 108907 & & OTY & 12 & 07 & & \\
\hline 039 & 01 & 108952 & 108952 & 0 TY & 12 & 07 & & \\
\hline 039 & 01 & 109 & & OTY & 12 & 07 & & \\
\hline 039 & 01 & 117817 & & OTY & 12 & 07 & & \\
\hline 039 & 01 & 123386 & & OTY & 12 & 07 & & \\
\hline 039 & 01 & 125 & & OTY & 12 & 07 & & \\
\hline 039 & 01 & 127184 & & OTY & 12 & 07 & & \\
\hline 039 & 01 & 136 & 136 & OTY & 12 & 07 & & \\
\hline 039 & 01 & 139 & 139 & OTY & 12 & 07 & & \\
\hline 039 & 01 & 1746016 & & OTY & 12 & 07 & & \\
\hline 039 & 01 & 18540299 & & OTY & 12 & 07 & & \\
\hline 039 & 01 & 195 & 195 & OTY & 12 & 07 & & \\
\hline 039 & 01 & 198 & 198 & OTY & 12 & 07 & & \\
\hline 039 & 01 & 199 & 199 & OTY & 12 & 07 & & \\
\hline 039 & 01 & 226 & 226 & OTY & 12 & 07 & & \\
\hline 039 & 01 & 246 & & OTY & 12 & 07 & & \\
\hline 039 & 01 & 253 & 253 & OTY & 12 & 07 & & \\
\hline 039 & 01 & 51285 & & OTY & 12 & 07 & & \\
\hline 039 & 01 & 56235 & 56235 & OTY & 12 & 07 & & \\
\hline 039 & 01 & 624 & & 0 TY & 12 & 07 & & \\
\hline 039 & 01 & 67663 & & OTY & 12 & 07 & & \\
\hline 039 & 01 & 71556 & & OTY & 12 & 07 & & \\
\hline 039 & 01 & 74839 & & OTY & 12 & 07 & & \\
\hline 039 & 01 & 74873 & & $0 T Y$ & 12 & 07 & & \\
\hline 039 & 01 & 75014 & & OTY & 12 & 07 & & \\
\hline 039 & 01 & 75070 & 75070 & OTY & 12 & 07 & & \\
\hline 039 & 01 & 75092 & & OTY & 12 & 07 & & \\
\hline 039 & 01 & 7723140 & 7723140 & OTY & 12 & 07 & & \\
\hline 039 & 01 & 78875 & & OTY & 12 & 07 & & \\
\hline 039 & 01 & 78933 & & OTY & 12 & 07 & & \\
\hline 039 & 01 & 79016 & & 0 TY & 12 & 07 & & \\
\hline 039 & 01 & 87865 & & 0 TY & 12 & 07 & & \\
\hline 039 & 01 & 88062 & & OTY & 12 & 07 & & \\
\hline 039 & 01 & 92 & & OTY & 12 & 07 & & \\
\hline 039 & 01 & 93 & & OTY & 12 & 07 & & \\
\hline 039 & 01 & 95476 & & OTY & 12 & 07 & & \\
\hline 039 & 01 & 98862 & & OTY & 12 & 07 & & \\
\hline 039 & 01 & $B Z$ & 71432 & OTY & 12 & 07 & & \\
\hline 039 & 01 & $\mathrm{CL}$ & 7782505 & OTY & 12 & 07 & & \\
\hline 039 & 01 & $\mathrm{CO}$ & 630080 & OTY & 12 & 07 & & \\
\hline 039 & 01 & FORM & 50000 & OTY & 12 & 07 & & \\
\hline 039 & 01 & $\mathrm{HCL}$ & 7647010 & OTY & 12 & 07 & & \\
\hline 039 & 01 & NO2 & 10102440 & OTY & 12 & 07 & & \\
\hline 039 & 01 & PM10 & & OTY & 12 & 07 & & \\
\hline 039 & 01 & PM25 & & OTY & 12 & 07 & & \\
\hline 039 & 01 & PT & & OTY & 12 & 07 & & \\
\hline 039 & 01 & $\mathrm{SO} 2$ & 7446095 & OTY & 12 & 07 & & \\
\hline 039 & 01 & TOLU & 108883 & $0 T Y$ & 12 & 07 & & \\
\hline 039 & 01 & VOC & & OTY & 12 & 07 & & \\
\hline 040 & 01 & 100027 & & 0 TY & 12 & 07 & & \\
\hline 040 & 01 & 100414 & 100414 & OTY & 12 & 07 & & \\
\hline 040 & 01 & 100425 & $x$ & OTY & 12 & 07 & & \\
\hline
\end{tabular}




\begin{tabular}{|c|c|c|c|c|c|c|}
\hline 040 & 01 & 106990 & & $0 \mathrm{TY}$ & 12 & 07 \\
\hline 040 & 01 & 107028 & & OTY & 12 & 07 \\
\hline 040 & 01 & 107062 & & OTY & 12 & 07 \\
\hline 040 & 01 & 108907 & & $0 T Y$ & 12 & 07 \\
\hline 040 & 01 & 108952 & 108952 & $0 T Y$ & 12 & 07 \\
\hline 040 & 01 & 109 & & O TY & 12 & 07 \\
\hline 040 & 01 & 117817 & & O TY & 12 & 07 \\
\hline 040 & 01 & 123386 & & 0 TY & 12 & 07 \\
\hline 040 & 01 & 125 & & OTY & 12 & 07 \\
\hline 040 & 01 & 127184 & & OTY & 12 & 07 \\
\hline 040 & 01 & 136 & 136 & OTY & 12 & 07 \\
\hline 040 & 01 & 139 & 139 & 0 TY & 12 & 07 \\
\hline 040 & 01 & 1746016 & & OTY & 12 & 07 \\
\hline 040 & 01 & 18540299 & & 0 TY & 12 & 07 \\
\hline 040 & 01 & 195 & 195 & 0 TY & 12 & 07 \\
\hline 040 & 01 & 198 & 198 & $0 \mathrm{TY}$ & 12 & 07 \\
\hline 040 & 01 & 199 & 199 & $0 T Y$ & 12 & 07 \\
\hline 040 & 01 & 226 & 226 & OTY & 12 & 07 \\
\hline 040 & 01 & 246 & & $0 T Y$ & 12 & 07 \\
\hline 040 & 01 & 253 & 253 & $0 \mathrm{TY}$ & 12 & 07 \\
\hline 040 & 01 & 51285 & & 0 TY & 12 & 07 \\
\hline 040 & 01 & 56235 & 56235 & 0 TY & 12 & 07 \\
\hline 040 & 01 & 624 & & $0 \mathrm{TY}$ & 12 & 07 \\
\hline 040 & 01 & 67663 & & $0 T Y$ & 12 & 07 \\
\hline 040 & 01 & 71556 & & $0 T Y$ & 12 & 07 \\
\hline 040 & 01 & 74839 & & $0 \mathrm{TY}$ & 12 & 07 \\
\hline 040 & 01 & 74873 & & $0 \mathrm{TY}$ & 12 & 07 \\
\hline 040 & 01 & 75014 & & $0 T Y$ & 12 & 07 \\
\hline 040 & 01 & 75070 & 75070 & $0 T Y$ & 12 & 07 \\
\hline 040 & 01 & 75092 & & $0 \mathrm{TY}$ & 12 & 07 \\
\hline 040 & 01 & 7723140 & 7723140 & $0 \mathrm{TY}$ & 12 & 07 \\
\hline 040 & 01 & 78875 & & $0 T Y$ & 12 & 07 \\
\hline 040 & 01 & 78933 & & $0 \mathrm{TY}$ & 12 & 07 \\
\hline 040 & 01 & 79016 & & $0 T Y$ & 12 & 07 \\
\hline 040 & 01 & 87865 & & $0 \mathrm{TY}$ & 12 & 07 \\
\hline 040 & 01 & 88062 & & $0 T Y$ & 12 & 07 \\
\hline 040 & 01 & 92 & & $0 T Y$ & 12 & 07 \\
\hline 040 & 01 & 93 & & $0 \mathrm{TY}$ & 12 & 07 \\
\hline 040 & 01 & 95476 & & $0 \mathrm{TY}$ & 12 & 07 \\
\hline 040 & 01 & 98862 & & $0 T Y$ & 12 & 07 \\
\hline 040 & 01 & $B Z$ & 71432 & $0 T Y$ & 12 & 07 \\
\hline 040 & 01 & $\mathrm{CL}$ & 7782505 & $0 \mathrm{TY}$ & 12 & 07 \\
\hline 040 & 01 & $\mathrm{CO}$ & 630080 & 0 TY & 12 & 07 \\
\hline 040 & 01 & FORM & 50000 & $0 \mathrm{TY}$ & 12 & 07 \\
\hline 040 & 01 & $\mathrm{HCL}$ & 7647010 & $0 \mathrm{TY}$ & 12 & 07 \\
\hline 040 & 01 & NO2 & 10102440 & $0 \mathrm{TY}$ & 12 & 07 \\
\hline 040 & 01 & PM10 & & $0 T Y$ & 12 & 07 \\
\hline 040 & 01 & PM25 & & $0 T Y$ & 12 & 07 \\
\hline 040 & 01 & PT & & $0 \mathrm{TY}$ & 12 & 07 \\
\hline 040 & 01 & $\mathrm{SO} 2$ & 7446095 & $0 T Y$ & 12 & 07 \\
\hline 040 & 01 & TOLU & 108883 & $0 T Y$ & 12 & 07 \\
\hline 040 & 01 & VOC & & $0 T Y$ & 12 & 07 \\
\hline 041 & 01 & 100027 & & $0 \mathrm{TY}$ & 12 & 07 \\
\hline 041 & 01 & 100414 & 100414 & $0 \mathrm{TY}$ & 12 & 07 \\
\hline 041 & 01 & 100425 & & $0 T Y$ & 12 & 07 \\
\hline 041 & 01 & 106990 & & 0 TY & 12 & 07 \\
\hline 041 & 01 & 107028 & & $0 \mathrm{TY}$ & 12 & 07 \\
\hline 041 & 01 & 107062 & & $0 \mathrm{TY}$ & 12 & 07 \\
\hline 041 & 01 & 108907 & & $0 T Y$ & 12 & 07 \\
\hline 041 & 01 & 108952 & 108952 & $0 T Y$ & 12 & 07 \\
\hline 041 & 01 & 109 & & $0 \mathrm{TY}$ & 12 & 07 \\
\hline 041 & 01 & 117817 & & $0 \mathrm{TY}$ & 12 & 07 \\
\hline 041 & 01 & 123386 & & $0 \mathrm{TY}$ & 12 & 07 \\
\hline 041 & 01 & 125 & & $0 \mathrm{TY}$ & 12 & 07 \\
\hline 041 & 01 & 127184 & & $0 T Y$ & 12 & 07 \\
\hline 041 & 01 & 136 & 136 & $0 T Y$ & 12 & 07 \\
\hline 041 & 01 & 139 & 139 & $0 \mathrm{TY}$ & 12 & 07 \\
\hline 041 & 01 & 1746016 & & $0 \mathrm{TY}$ & 12 & 07 \\
\hline 041 & 01 & 18540299 & & $0 T Y$ & 12 & 07 \\
\hline 041 & 01 & 195 & 195 & $0 \mathrm{TY}$ & 12 & 07 \\
\hline 041 & 01 & 198 & 198 & $0 \mathrm{TY}$ & 12 & 07 \\
\hline 041 & 01 & 199 & 199 & $0 \mathrm{TY}$ & 12 & 07 \\
\hline 041 & 01 & 226 & 226 & 0 TY & 12 & 07 \\
\hline 041 & 01 & 246 & & OTY & 12 & 07 \\
\hline 041 & 01 & 253 & 253 & $0 \mathrm{TY}$ & 12 & 07 \\
\hline 041 & 01 & 51285 & & $0 \mathrm{TY}$ & 12 & 07 \\
\hline 041 & 01 & 56235 & 56235 & $0 T Y$ & 12 & 07 \\
\hline 041 & 01 & 624 & & $0 \mathrm{TY}$ & 12 & 07 \\
\hline 041 & 01 & 67663 & 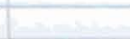 & OTY & 12 & 07 \\
\hline 041 & 01 & 71556 & & $0 \mathrm{TY}$ & 12 & 07 \\
\hline
\end{tabular}




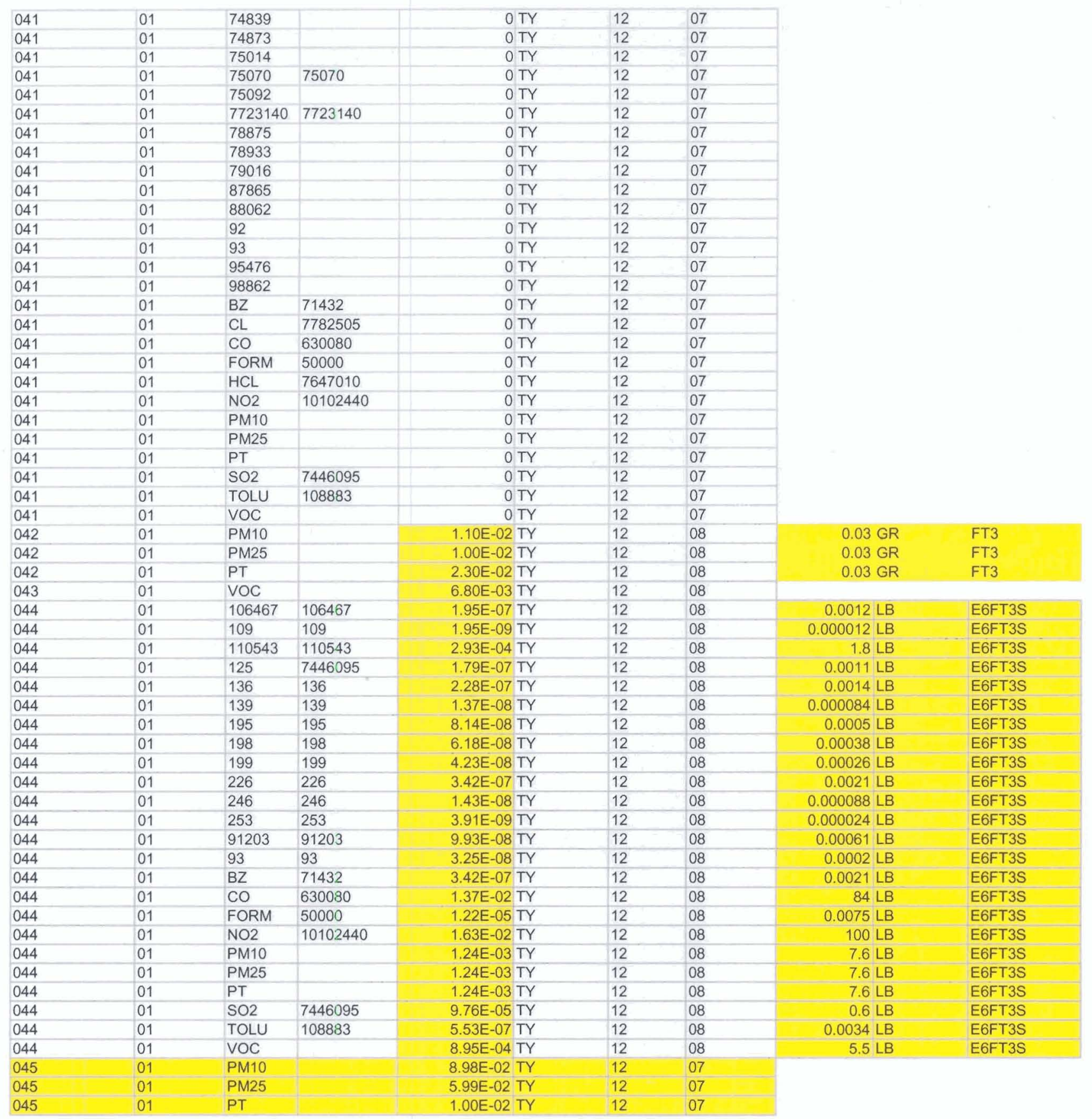




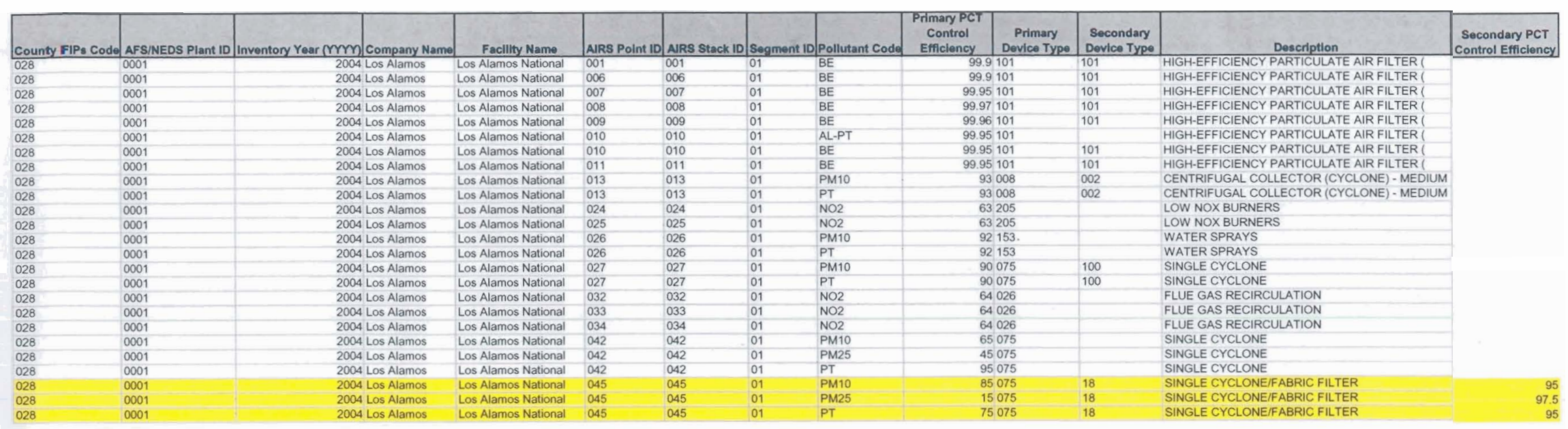



ATTACHMENT C.

2004 SEMI-ANNUAL EMISSIONS REPORTS SUBMITTED

UNDER TITLE V OPERATING PERMIT REQUIREMENTS 


\section{Los Alamos \\ NATIONAL LAEORATORY}

Associate Director for Operations

P.O. Box 1663, A 104

Los Alamos, New Mexico 87545

Date: September 24, 2004

505-667-0079/Fax 505-665-1812

Refer To: AD-Ops:04-109

Dr. John Volkerding

Program Manager, Compliance \& Enforcement Section

New Mexico Environment Department

Air Quality Bureau

2048 Galisteo Street

Santa Fe, NM 87505

\section{SUBJECT: SEMI-ANNUAL EMISSIONS REPORT}

Dear Dr. Volkerding:

Attached you will find the semi-annual emission report for January through June of 2004. This report includes actual emissions from permitted sources included in section 2.0 of the Los Alamos National Laboratory (LANL) Operating Permit (Permit Number: P100). This submission satisfies permit condition 4. 1., which requires submission of a semi-annual emissions report on a 6-month basis. Furthermore, this report is submitted within 90 days from the end of the reporting period as stipulated in permit condition 4.3 .

In this report, actual emissions are listed along with the permit limits for ease in comparing and verifying compliance. No emission limits were exceeded during this reporting period. Emissions are also reported from boiler and generator insignificant sources. These sources are included to demonstrate that LANL has not exceeded Prevention of Significant Deterioration (PSD) applicability thresholds.

Should you have any questions or comments regarding the information provided in this report, please contact Steve Story at (505) 665-2169.

Sincerely,

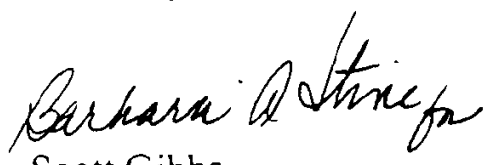

Scott Gibbs

Acting Associate Director for Operations

WSG/DLP:alb

Att: $a / s$

Cy:

S. Fong, DOE-LA-AO, A316

B. Ramsey, RRES-DO, J59I

D. Stavert, RRES-EP, J591 
V. George, RRES-DO, J591

$M$. Reed, RRES-DO, J556

J. Dewart, RRES-MAQ, J978

D. Wilburn, RRES-MAQ, $J 978$

S. Story, RRES-MAQ, J978

J. Hurtle, RRES-MAQ, J978

D. Paulson, RRES-MAQ, $J 978$

P. Wardwell, LC-ESH, A 187

RRES-MAQ Title-V Reports File

RRES-MAQ File, $\mathrm{J} 978$

ADO-Ops File 


\section{Los Alamos National Laboratory \\ 2004 Semi-Annual Emissions Report \\ (January through June)}

This report is being provided to meet the requirement set forth in permit condition 4.1 of the Los Alamos National Laboratory (LANL) Operating Permit Number $\mathrm{P} 100$. The emissions included in this report were calculated using operating data recorded during the first six months of 2004

\section{Facility Emissions}

The following table displays the actual facility-wide emissions compared with the Facility Wide Emission Limits specified in permit condition 2.11 of the Operating Permit. These emissions include insignificant sources, which are included to demonstrate that facility-wide emissions are below all PSD applicability threshold limits.

\begin{tabular}{|l|c|c|c|c|}
\hline \multicolumn{1}{|c|}{ Pollutant } & $\begin{array}{c}\text { January - June } \\
\text { Emissions } \\
\text { (tons) }\end{array}$ & $\begin{array}{c}\text { July - December } \\
\text { Emissions } \\
\text { (tons) }\end{array}$ & $\begin{array}{c}\text { 2004 Annual Emissions } \\
\text { (tons) }\end{array}$ & $\begin{array}{c}\text { Facility Wide Emission Limits } \\
\text { (Permit Condition 2.11) } \\
\text { (tons per year) }\end{array}$ \\
\hline Nitrogen Oxides (NOx) & 26.6 & & & 245 \\
\hline Carbon Monoxide (CO) & 19.1 & & & 225 \\
\hline Volatile Organic Compounds (VOCs) & 6.7 & & & 200 \\
\hline Sulfur Dioxide (SO ) & 0.7 & & & 150 \\
\hline Particulate Matter (PM) & 2.5 & & & 120 \\
\hline Hazardous Air Pollutants (HAPs) & 4.9 & & & 24 combined \\
\hline $\begin{array}{l}\text { Jan-June Highest Individual HAP } \\
\text { (Hydrochloric Acid) }\end{array}$ & 1.2 & & 8 individual \\
\hline
\end{tabular}


Los Alamos National Laboratory

\section{Semi-Annual Emissions Report}

\section{(January through June)}

\section{Source Emissions}

The following are the actual emissions from permitted sources listed in permit condition 2.0 of the operating permit for the six month reporting period. Included with these emissions are the source specific emission limits if applicable.

\section{Permit Condition/Source}

2.1 Asphalt Production - Asphalt Plant located at TA-60

\begin{tabular}{|l|c|c|c|c|}
\hline \multicolumn{1}{|c|}{ Pollutant } & $\begin{array}{c}\text { Jan-June } \\
\text { Emissions } \\
\text { (tons) }\end{array}$ & $\begin{array}{c}\text { July-Dec } \\
\text { Emissions } \\
\text { (tons) }\end{array}$ & $\begin{array}{c}\text { Annual Emissions } \\
\text { (tons) }\end{array}$ & $\begin{array}{c}\text { Permit Limits } \\
\text { (Permit Condition } \\
\text { 2.1.2) } \\
\text { (tons per year) }\end{array}$ \\
\hline NOx & 0.0 & & & 1.0 \\
\hline $\mathrm{SO}_{2}$ & 0.0 & & & 1.0 \\
\hline $\mathrm{PM}$ & 0.0 & & & $* 35.4 \mathrm{lbs} / \mathrm{hr}$ \\
\hline $\mathrm{CO}$ & 0.0 & & & 2.6 \\
\hline VOC & 0.0 & & & 1.0 \\
\hline HAPs & 0.0 & & & $\begin{array}{c}\text { No Source Permit } \\
\text { Limit }\end{array}$ \\
\hline
\end{tabular}

Note: The Asphalt Plant did not operate during the first 6 months of 2004.

* The Asphal Plant does not have a tons per year limit for PM. The lb/hr emissions will be demonstrated during the initial source compliance test. 


\section{Los Alamos National Laboratory \\ 2004 Semi-Annual Emissions Report \\ (January through June)}

2.2 Beryllium Activities

\begin{tabular}{|c|c|c|c|c|c|}
\hline Source & Pollutant & $\begin{array}{c}\text { Jan-June } \\
\text { Emissions } \\
\text { (grams) }\end{array}$ & $\begin{array}{c}\text { July-Dec } \\
\text { Emissions } \\
\text { (grams) }\end{array}$ & $\begin{array}{c}\text { Annual } \\
\text { Emissions } \\
\text { (grams) }\end{array}$ & $\begin{array}{c}\text { Permit Limits } \\
\text { (Permit Condition } \\
\text { 2.2.2) }\end{array}$ \\
\hline $\begin{array}{c}\text { Beryllium Test Facility } \\
\text { TA-3-141 }\end{array}$ & Beryllium & $3.30 \mathrm{E}-03$ & & $3.5 \mathrm{gm} / \mathrm{yr}$ \\
\hline $\begin{array}{c}\text { Target Fabrication Facility } \\
\text { TA-35-213 }\end{array}$ & Beryllium & 0.04 & & $0.36 \mathrm{gm} / \mathrm{yr}$ \\
\hline $\begin{array}{c}\text { Plutonium Facility } \\
\text { TA-55-PF4 }\end{array}$ & Beryllium & 1.495 & & & \\
\cline { 2 - 6 } $\begin{array}{c}\text { Machining Operation } \\
\text { Foundry Operation }\end{array}$ & Aluminum & 1.495 & & & $2.99 \mathrm{gm} / \mathrm{yr}$ \\
\cline { 2 - 6 } & Beryllium & 0 & & & $2.99 \mathrm{gm} / \mathrm{yr}$ \\
\cline { 2 - 6 } & Aluminum & 0 & & $8.73 \times 10^{-4} \mathrm{gm} / \mathrm{yr}$ \\
\hline \multicolumn{2}{|c|}{ Jan-June Beryllium Total (tons) $=$} & $1.70 \mathrm{E}-06$ & Jan-June Alumimum Total (tons) $=$ & $8.73 \times 10^{-4} \mathrm{gm} / \mathrm{yr}$ \\
\hline
\end{tabular}

Note: Emission values shown for the Beryllium Test Facility are from actual stack emission measurements. Emissions for the Target Facility are from initial compliance testing of that source. Emissions for the Plutonium Facility are based on permitted limits. The Plutonium Facility foundry operations did not operate during the first six months of 2004. Other sources listed in section 2.2 of the permit do not require reporting in the Semi-Annual Emissions Report.

\subsection{Boilers and Heaters}

\begin{tabular}{|l|c|c|c|c|}
\hline Pollutant & $\begin{array}{c}\text { January - June } \\
\text { Emissions } \\
\text { (tons) }\end{array}$ & $\begin{array}{c}\text { July - December } \\
\text { Emissions } \\
\text { (tons) }\end{array}$ & $\begin{array}{c}\text { Annual Emissions } \\
\text { (tons) }\end{array}$ & $\begin{array}{c}\text { Permit Limits } \\
\text { (Permit Condition } \\
\text { 2.3.2) } \\
\text { (tons per year) }\end{array}$ \\
\hline $\mathrm{NOx}$ & 15.4 & & & 80 \\
\hline $\mathrm{SO}_{2}$ & 0.1 & & & 50 \\
\hline $\mathrm{PM}$ & 1.2 & & & 50 \\
\hline PM-10 & 1.2 & & & 50 \\
\hline $\mathrm{CO}$ & 12.5 & & & 80 \\
\hline VOC & 0.9 & & & 50 \\
\hline HAPS & 0.29 & & & No Source Limit \\
\hline
\end{tabular}

Note: The emissions shown in this table include significant and insignificant sources. This section does not include the TA-3-22 Power Plant boilers. These can be found under 2.9. The TA-21 steam plant boilers are included in this table. 
Los Alamos National Laboratory

2004 Semi-Annual Emissions Report

(January through June)

\subsection{Carpenter Shops}

\begin{tabular}{|c|c|c|c|c|c|}
\hline Shop & Pollutant & $\begin{array}{c}\text { January - June } \\
\text { Emissions } \\
\text { (tons) }\end{array}$ & $\begin{array}{c}\text { July - December } \\
\text { Emission } \\
\text { (tons) }\end{array}$ & $\begin{array}{c}\text { Annual Emissions } \\
\text { (tons) }\end{array}$ & $\begin{array}{c}\text { Permit Limit } \\
\text { (Permit Condition } \\
\text { 2.42) } \\
\text { (tons per year) }\end{array}$ \\
\hline TA-3-38 & $\mathrm{PM}_{10}$ & 0.016 & & & 3.07 \\
\hline TA-15-563 & $\mathrm{PM}_{10}$ & 0 & & & 2.81 \\
\hline
\end{tabular}

Note: The TA-15-563 Carpenter Shop did not operate during the first 6 months of the year.

2.5 Chemical Usage

\begin{tabular}{|c|c|c|c|c|}
\hline Pollutant & $\begin{array}{l}\text { January - June } \\
\text { Emissions } \\
\text { (tons) }\end{array}$ & $\begin{array}{l}\text { July - December } \\
\text { Emissions } \\
\text { (tons) }\end{array}$ & $\begin{array}{c}\text { Annual Emissions } \\
\text { (tons) }\end{array}$ & $\begin{array}{c}\text { Permit Limits } \\
\text { (Permit Condition } \\
\text { 2.5.3.1) }\end{array}$ \\
\hline vocs & 4.9 & & & \multirow{3}{*}{$\begin{array}{c}\text { Source limits refer } \\
\text { to facility wide } \\
\text { limits. } \\
\text { (See Facility } \\
\text { Emissions Table } \\
\text { on Page 1) }\end{array}$} \\
\hline HAPS & 4.3 & & & \\
\hline $\begin{array}{l}\text { Highest Individual HAP } \\
\text { (Hydrochloric Acid) }\end{array}$ & 1.2 & & & \\
\hline
\end{tabular}

2.6 Degreasers

\begin{tabular}{|c|c|c|c|c|}
\hline $\begin{array}{l}\text { Degreaser } \\
\text { TA-55-DG-1 }\end{array}$ & $\begin{array}{l}\text { January - June } \\
\text { Emissions } \\
\text { (tons) }\end{array}$ & $\begin{array}{l}\text { July - December } \\
\text { Emissions } \\
\text { (tons) }\end{array}$ & $\begin{array}{c}\text { Annual Emissions } \\
\text { (tons) }\end{array}$ & $\begin{array}{c}\text { Permit Limits } \\
\text { (Permit Condition } \\
\text { 2.6.2.1) (tons per } \\
\text { year) }\end{array}$ \\
\hline Vocs & 0.004 & & & \multirow{2}{*}{$\begin{array}{l}\text { Source limits refer } \\
\text { to facility wide } \\
\text { limits. (See Facility } \\
\text { Emissions Table } \\
\text { on Page 1) }\end{array}$} \\
\hline HAPs & 0.004 & & & \\
\hline
\end{tabular}

Note: Degreasers TA-55-DG-2 and TA-55-DG-3 were not used in the first six months of 2004. These degreasers are not expected to be used in the near future and are in storage. 
Los Alamos National Laboratory 2004 Semi-Annual Emissions Report

(January through June)

2.7 Internal Combustion Sources

\begin{tabular}{|c|c|c|c|c|}
\hline $\begin{array}{c}\text { Generator } \\
\text { TA-33-G-1 }\end{array}$ & $\begin{array}{c}\text { Jan-June } \\
\text { Emissions } \\
\text { (tons) }\end{array}$ & $\begin{array}{c}\text { July-Dec } \\
\text { Emissions } \\
\text { (tons) }\end{array}$ & $\begin{array}{c}\text { Permit Limits } \\
\text { Annual } \\
\text { (tons) }\end{array}$ & $\begin{array}{c}\text { (Permit Condition } \\
\text { 2.7.2) } \\
\text { (tons per year) }\end{array}$ \\
\hline TSP & 0.0 & & & 0.6 \\
\hline PM $_{10}$ & 0.0 & & & 0.6 \\
\hline NOx & 0.0 & & & 18.1 \\
\hline CO & 0.0 & & & 15.2 \\
\hline VOC & 0.0 & & & 0.3 \\
\hline SOX & 0.0 & & & 2.5 \\
\hline HAPS & 0.0 & & & No Source Limit \\
\hline
\end{tabular}

Note: The TA-33-G-1 generator did not operate during the first six months of 2004.

\begin{tabular}{|c|c|c|c|c|}
\hline Standby Generators & $\begin{array}{c}\text { Jan-June } \\
\text { Emissions } \\
\text { (tons) }\end{array}$ & $\begin{array}{c}\text { July-Dec } \\
\text { Emissions } \\
\text { (tons) }\end{array}$ & $\begin{array}{c}\text { Permit Limits } \\
\text { Annual } \\
\text { (tons) }\end{array}$ & $\begin{array}{c}\text { (Permit Condition } \\
\text { 2.7.2) }\end{array}$ \\
\hline TSP & 0.1 & & & No Source Specific \\
\hline PM $_{10}$ & 0.1 & & & $\begin{array}{c}\text { Emission Limits for } \\
\text { Standby } \\
\text { Generators }\end{array}$ \\
\hline NOx & 2.3 & & & \\
\hline CO & 0.6 & & & \\
\hline VOC & 0.1 & & & \\
\hline SOX & 0.5 & & & \\
\hline
\end{tabular}

Note: Standby Generators are insignificant sources.

2.8 Paper Shredder

\begin{tabular}{|c|c|c|c|c|}
\hline $\begin{array}{c}\text { Emission Unit } \\
\text { TA-52-11 }\end{array}$ & $\begin{array}{c}\text { January - June } \\
\text { Emissions } \\
\text { (tons) }\end{array}$ & $\begin{array}{c}\text { July - December } \\
\text { Emissions } \\
\text { (tons) }\end{array}$ & $\begin{array}{c}\text { TSP Emission } \\
\text { Limit } \\
\text { (tons) } \\
\text { TSP }\end{array}$ & $\begin{array}{c}\text { (Permit Condition } \\
2.8 .2 .1) \\
\text { (tons per year) }\end{array}$ \\
\hline
\end{tabular}


Los Alamos National Laboratory

2004 Semi-Annual Emissions Report

(January through June)

2.9 Power Plant at Technical Area 3 (TA-3-22)

\begin{tabular}{|l|c|c|c|c|}
\hline Pollutant & $\begin{array}{c}\text { January - June } \\
\text { Emissions } \\
\text { (tons) }\end{array}$ & $\begin{array}{c}\text { July - December } \\
\text { Emissions } \\
\text { (tons) }\end{array}$ & $\begin{array}{c}\text { Annual Emissions } \\
\text { (tons) }\end{array}$ & $\begin{array}{c}\text { Permit Limit } \\
\text { (Permit Condition } \\
\text { 2.9.2) } \\
\text { (tons per year) }\end{array}$ \\
\hline NOX & 8.9 & & & 99.6 \\
\hline $\mathrm{SO}_{2}$ & 0.1 & & & 36.9 \\
\hline $\mathrm{TSP}$ & 1.2 & & & 15.7 \\
\hline PM & & & & 15.7 \\
\hline $\mathrm{CO}$ & 1.2 & & & 81.3 \\
\hline VOC & 6.1 & & & 11.1 \\
\hline HAPS & 0.8 & & & No Source Limit \\
\hline
\end{tabular}

2.10 Rock Crusher

The Rock Crusher was not used during this 6 month reporting period. The source was retired. 


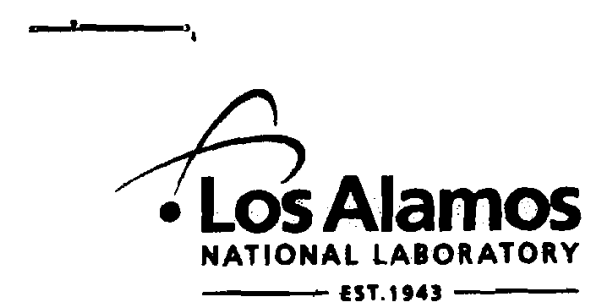

Associate Directorate for Technical Services

P.O. Box 1663, A 104

Los Alamos, New Mexico 87545

505-667-0079/Fax 505-665-1812

Date: March 23, 2005

Refer To: ADTS:05-023

Mr. Edward L. Horst

Environmental Compliance Specialist

Compliance \& Enforcement Section

New Mexico Environment Department

Air Quality Bureau

2048 Galisteo Street

Santa Fe, NM 87505

IDEA ID NO. 856 - LOS ALAMOS NATIONAL LABORATORY (LANL)
SEMI-ANNUAL EMISSIONS REPORT - OPERATING PERMIT NUMBER: P100

Dear Mr. Horst:

Attached is the semi-annual emissions report for July through December of 2004. This report includes actual emissions from permitted sources included in section 2.0 of the Los Alamos National Laboratory (LANL) Operating Permit. This submission satisfies permit condition 4.1., which requires submission of a semi-annual emissions report on a 6-month basis. Furthermore, this report is submitted within 90 days from the end of the reporting period as stipulated in permit condition 4.3.

In this report, actual emissions are listed along with the emission limits for ease in comparing and verifying compliance. No emission limits were exceeded during this reporting period. Emissions are also reported from insignificant boiler and generator sources. These sources are included to demonstrate that LANL has not exceeded Prevention of Significant Deterioration (PSD) applicability thresholds.

Also included are the more restrictive emission limits for the TA-3 power plant boilers, from NSR Air Quality Permit 2195BM1. This permit was issued on July 30, 2004, to install a combustion turbine at the power plant, but installation is not yet complete. In addition, emissions from the data disintegrator, which replaced the paper shredder in 2004, are included in this report. The emission limits for this unit were taken from NSR Air Quality Permit 2195-H. Both of these units will be incorporated into the LANL Operating Permit modification to be submitted by July 29, 2005.

Should you have any questions or comments regarding the information provided in this report, please contact Steve Story at (505) 665-2169.

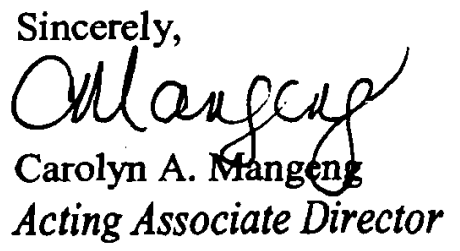




\section{CAM:alb}

Cy:

S. Fong, DOE-LA-AO, A316

K. Hargis, ENV-DO, J591

D. Stavert, ENV-DO, J591

J. Dewart, ENV-MAQ, J978

D. Wilburn, ENV-MAQ, J978

S. Story, ENV-MAQ, J978

J. Hurtle, ENV-MAQ, J978

D. Paulson, ENV-MAQ, J978

M. Stockton, ENV-MAQ, J978

P. Wardwell, LC-ESH, A187

ENV-MAQ Title-V Reports File

ENV-MAQ File 


\section{Title V Operating Permit Semi-Annual Emission Report}

July 1 - December 31,2004

\section{Identifing intomantio:}

Source Name: Los Alamos National Laboratory County: Los Alamos

Source Address:

City:

Los Alamos

State: $\mathbf{N M}$ Zip Code: 87545

Responsible Official: Carolyn A. Mangeng Ph No. (505) 667-0079 Fax No. (505) 665-1812

Technical Contact: Steven L. Story Ph No. (505) 665-2169 Fax No. (505) 665-8858

Principal Company Product or Business: National Security and Nuclear Weapons Research Primary SIC Code: 9711

Permit No. P100 IIDEATTempo ID No. 856\} Permit Issued Date: April 30,2004

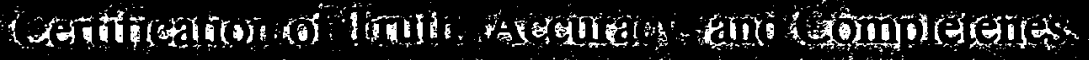

I, Carolyn A. Mangeng certify that, based on information and belief formed after reasonable inquiry, the statements and information in the attached semi-annual emission report are true, accurate, and complete.

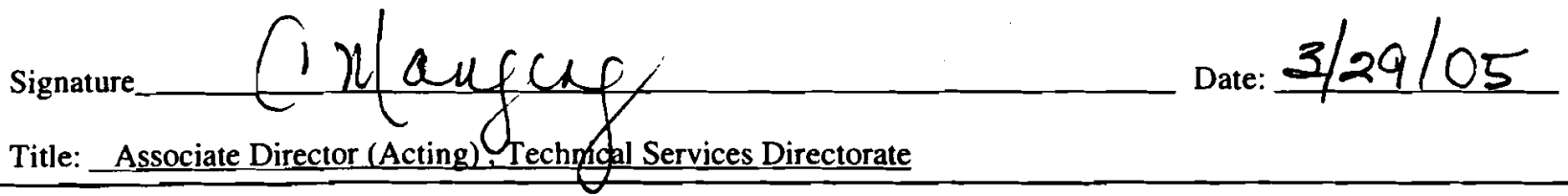




\section{Los Alamos National Laboratory \\ 2004 Semi-Annual Emissions Report}

(July through December)

This report is being provided to meet the requirement set forth in permit condition 4.1 of the Los Alamos National Laboratory (LANL) Operating Permit Number P100. The emissions were calculated using operating data recorded during the second six months of 2004 . The emissions from the first six months of 2004 were

submitted in the previous Semi-Annual Emissions Report, but are included here to calculate annual emissions.

\section{Facility Emissions}

The following table displays the actual facility-wide emissions compared with the facility wide emission limits specified in permit condition 2.11 of the Operating Permit. These emissions include insignificant sources, which are included to demonstrate that facility-wide emissions are below all PSD applicability threshold limits. Also, due to the method used for calculating Hazardous Air Pollutant (HAP) and Volitile Organic Compound (VOC) emissions from chemical use, fugitive emissions are included (see permit condition 4.1)

\begin{tabular}{|c|c|c|c|c|}
\hline Pollutant & $\begin{array}{c}\text { January - June } \\
\text { Emissions } \\
\text { (ton) } \\
\end{array}$ & $\begin{array}{c}\text { July - December } \\
\text { Emissions } \\
\text { (ton) }\end{array}$ & $\begin{array}{l}2004 \text { Annual Emissions } \\
\text { (ton) }\end{array}$ & $\begin{array}{c}\text { Facility Wide Emission Limits } \\
\text { (Permit Condition 2.11) } \\
\text { (ton per year) }\end{array}$ \\
\hline Nitrogen Oxides (NOx) & 26.5 & 24.0 & 50.5 & 245 \\
\hline Carbon Monoxide (CO) & 19.1 & 16.3 & 35.4 & 225 \\
\hline Volatile Organic Compounds (VOCs) & 6.7 & 4.7 & 11.4 & 200 \\
\hline Sulfur Dioxide $\left(\mathrm{SO}_{2}\right)$ & 0.7 & 0.8 & 1.5 & 150 \\
\hline Particulate Matter (PM) & 2.5 & 2.3 & 4.8 & 120 \\
\hline Hazardous Air Pollutants (HAPS) & 4.8 & 1.9 & 6.7 & 24 combined \\
\hline $\begin{array}{l}\text { Highest Individual HAP (Hydrochloric } \\
\text { Acid) }\end{array}$ & 1.2 & 0.3 & 1.5 & 8 individual \\
\hline
\end{tabular}


Los Alamos National Laboratory

2004 Semi-Annual Emissions Report

(July through December)

\section{Source Emissions}

The following are the actual emissions from permitted sources listed in permit condition 2.0 of the operating permit for the six month reporting period. Included with these emissions are the source specific emission limits when applicable.

\section{Permit Condition/Source}

2.1 Asphalt Production - Asphalt Plant located at TA-60

\begin{tabular}{|l|c|c|c|c|}
\hline \multicolumn{1}{|c|}{ Pollutant } & $\begin{array}{c}\text { Jan-June } \\
\text { Emissions } \\
\text { (ton) }\end{array}$ & $\begin{array}{c}\text { July-Dec } \\
\text { Emissions } \\
\text { (ton) }\end{array}$ & $\begin{array}{c}\text { Annual Emissions } \\
\text { (ton) }\end{array}$ & $\begin{array}{c}\text { Emission Limits } \\
\text { (Permit Condition } \\
\text { 2.1.2) } \\
\text { (ton per year) }\end{array}$ \\
\hline NOx & 0.0 & 0.0 & 0.0 & 1.0 \\
\hline $\mathrm{SO}_{2}$ & 0.0 & 0.0 & 0.0 & 1.0 \\
\hline $\mathrm{PM}$ & 0.0 & 0.0 & 0.0 & $* 35.4 \mathrm{lb} / \mathrm{hr}$ \\
\hline $\mathrm{CO}$ & 0.0 & 0.0 & 0.0 & 2.6 \\
\hline VOC & 0.0 & 0.0 & 0.0 & 1.0 \\
\hline HAPs & 0.0 & 0.0 & 0.0 & $\begin{array}{c}\text { No Source Permit } \\
\text { Limit }\end{array}$ \\
\hline
\end{tabular}

Note: The Asphalt Plant did not operate during 2004

The Asphalt Plant does not have a ton per year limit for PM. The lb/hr emissions will be

demonstrated during the initial source compliance test. 
Los Alamos National Laboratory 2004 Semi-Annual Emissions Report

(July through December)

2.2 Beryllium Activities

\begin{tabular}{|c|c|c|c|c|c|}
\hline Source & Pollutant & $\begin{array}{c}\text { Jan-June } \\
\text { Emissions } \\
\text { (gram) }\end{array}$ & $\begin{array}{c}\text { July-Dec } \\
\text { Emissions } \\
\text { (gram) }\end{array}$ & $\begin{array}{c}\text { Annual } \\
\text { Emissions (gram) }\end{array}$ & $\begin{array}{c}\text { Emission Limits } \\
\text { (Permit Condition } \\
2.2 .2)\end{array}$ \\
\hline $\begin{array}{c}\text { Beryllium Test Facility } \\
\text { TA-3-141 }\end{array}$ & Beryllium & $<0.0033$ & $<0.0032$ & $<0.0065$ & $3.5 \mathrm{gm} / \mathrm{yr}$ \\
\hline $\begin{array}{c}\text { Target Fabrication Facility } \\
\text { TA-35-213 }\end{array}$ & Beryllium & $<0.04$ & $<0.009$ & $<0.049$ & $0.36 \mathrm{gm} / \mathrm{yr}$ \\
\hline $\begin{array}{c}\text { Plutonium Facility } \\
\text { TA-55-PF4 } \\
\text { Machining Operation }\end{array}$ & Beryllium & $<1.495$ & $<1.41$ & $<2.905$ & $2.99 \mathrm{gm} / \mathrm{yr}$ \\
\cline { 2 - 6 } & Aluminum & $<1.495$ & $<1.41$ & $<2.905$ & $2.99 \mathrm{gm} / \mathrm{yr}$ \\
\cline { 2 - 6 } Foundry Operation & Beryllium & 0 & 0 & 0 & $8.73 \times 10^{-4} \mathrm{gm} / \mathrm{yr}$ \\
\cline { 2 - 6 } & Aluminum & 0 & 0 & 0 & $8.73 \times 10^{-4} \mathrm{gm} / \mathrm{yr}$ \\
\hline \multicolumn{2}{|c|}{ Beryllium Total (tons) $=$} & $<1.70 \times 10^{-6}$ & $<1.57 \times 10^{-6}$ & $<3.27 \times 10^{-6}$ & \\
\hline
\end{tabular}

Note: Emission values shown for the Beryllium Test Facility are from actual stack emission measurements. Emissions for the Target Fabrication Facility are based on initial compliance testing of that source and a conservative use of maximum hours of operation. Emissions for the Plutonium Facility Machining Operation are based on initial compliance testing of the source and a conservative use of maximum throughput. The Plutonium Facility Foundry Operations did not operate during 2004. Other beryllium sources listed in section 2.2 of the permit do not require reporting in the Semi-Annual Emissions Report

2.3 Boilers and Heaters

\begin{tabular}{|l|c|c|c|c|}
\hline Pollutant & $\begin{array}{c}\text { January - June } \\
\text { Emissions } \\
\text { (ton) }\end{array}$ & $\begin{array}{c}\text { July - December } \\
\text { Emissions } \\
\text { (ton) }\end{array}$ & $\begin{array}{c}\text { Annual Emissions } \\
\text { (ton) }\end{array}$ & $\begin{array}{c}\text { Emission Limits } \\
\text { (Permit Condition } \\
\text { 2.3.2) } \\
\text { (ton per year) }\end{array}$ \\
\hline NOx & 15.50 & 12.80 & 28.30 & 80 \\
\hline $\mathrm{SO}_{2}$ & 0.10 & 0.08 & 0.18 & 50 \\
\hline $\mathrm{PM}$ & 1.20 & 1.03 & 2.23 & 50 \\
\hline PM-10 & 1.20 & 1.03 & 2.23 & 50 \\
\hline $\mathrm{CO}$ & 12.50 & 10.25 & 22.75 & 80 \\
\hline VOC & 0.90 & 0.71 & 1.61 & 50 \\
\hline HAPS & 0.26 & 0.24 & 0.50 & No Source Limit \\
\hline
\end{tabular}

Note: The emissions shown in this table include significant and insignificant sources. This section does not

include the TA-3-22 Power Plant boilers (see section 29). The TA-21 steam plant bolers are included in th table. 
Los Alamos National Laboratory

2004 Semi-Annual Emissions Report

(July through December)

2.4 Carpenter Shops

\begin{tabular}{|c|c|c|c|c|c|}
\hline Shop & Pollutant & $\begin{array}{c}\text { January - June } \\
\text { Emissions } \\
\text { (ton) }\end{array}$ & $\begin{array}{c}\text { July - December } \\
\text { Emission } \\
\text { (ton) }\end{array}$ & $\begin{array}{c}\text { Annual Emissions } \\
\text { (ton) }\end{array}$ & $\begin{array}{c}\text { Emission Limits } \\
\text { (Permit Condition } \\
\text { 2.4.2) } \\
\text { (ton per year) }\end{array}$ \\
\hline TA-3-38 & $\mathrm{PM}_{10}$ & 0.016 & 0.005 & 0.021 & 3.07 \\
\hline TA-15-563 & $\mathrm{PM}_{10}$ & 0 & 0 & 0 & 2.81 \\
\hline
\end{tabular}

Note: The TA-15-563 Carpenter Shop did not operate during 2004

2.5 Chemical Usage

\begin{tabular}{|c|c|c|c|c|}
\hline Pollutant & $\begin{array}{l}\text { January - June } \\
\text { Emissions } \\
\text { (ton) }\end{array}$ & $\begin{array}{l}\text { July - December } \\
\text { Emissions } \\
\text { (ton) }\end{array}$ & $\begin{array}{l}\text { Annual Emissions } \\
\text { (ton) }\end{array}$ & $\begin{array}{c}\text { Emission Limits } \\
\text { (Permit Condition } \\
\text { 2.5.3.1) }\end{array}$ \\
\hline Vocs & 4.91 & 3.04 & 7.95 & \multirow{3}{*}{$\begin{array}{l}\text { Source limits refer } \\
\text { to facility wide } \\
\text { limits. } \\
\text { (See Facility } \\
\text { Emissions Table on } \\
\text { Page 1) }\end{array}$} \\
\hline HAPs & 4.28 & 1.44 & 5.72 & \\
\hline $\begin{array}{l}\text { Highest Individual HAP } \\
\text { (Hydrochloric Acid) }\end{array}$ & 1.19 & 0.32 & 1.51 & \\
\hline
\end{tabular}

2.6 Degreasers

\begin{tabular}{|c|c|c|c|c|}
\hline $\begin{array}{c}\text { Degreaser } \\
\text { TA-55-DG-1 }\end{array}$ & $\begin{array}{c}\text { January - June } \\
\text { Emissions } \\
\text { (ton) }\end{array}$ & $\begin{array}{c}\text { July - December } \\
\text { Emissions } \\
\text { (ton) }\end{array}$ & $\begin{array}{c}\text { Annual Emissions } \\
\text { (ton) }\end{array}$ & $\begin{array}{c}\text { Emission Limits } \\
\text { (Permit Condition } \\
2.6 .2 .1 \text { ) (ton per } \\
\text { year) }\end{array}$ \\
\hline vocs & 0.004 & 0.007 & 0.011 & $\begin{array}{c}\text { Source limits refer } \\
\text { to facility wide } \\
\text { limits. (See Facility } \\
\text { Emissions Table on } \\
\text { Page 1) }\end{array}$ \\
\hline
\end{tabular}

Note: Degreasers TA-55-DG-2 and TA-55-DG-3 were not used in 2004. These degreasers are not expected

to be used in the near future and are in storage. 
Los Alamos National Laboratory 2004 Semi-Annual Emissions Report

(July through December)

2.7 Internal Combustion Sources

\begin{tabular}{|c|c|c|c|c|}
\hline $\begin{array}{c}\text { Generator } \\
\text { TA-33-G-1 }\end{array}$ & $\begin{array}{c}\text { Jan-June } \\
\text { Emissions } \\
\text { (ton) }\end{array}$ & $\begin{array}{c}\text { July-Dec } \\
\text { Emissions } \\
\text { (ton) }\end{array}$ & $\begin{array}{c}\text { Annual } \\
\text { (ton) }\end{array}$ & $\begin{array}{c}\text { Emission Limits } \\
\text { (Permit Condition } \\
\text { 2.7.2) } \\
\text { (ton per year) }\end{array}$ \\
\hline TSP & 0.0 & 0.0 & 0.0 & 0.6 \\
\hline PM $_{10}$ & 0.0 & 0.0 & 0.0 & 0.6 \\
\hline NOx & 0.0 & 0.0 & 0.0 & 18.1 \\
\hline CO & 0.0 & 0.0 & 0.0 & 15.2 \\
\hline VOC & 0.0 & 0.0 & 0.0 & 0.3 \\
\hline SO & 0.0 & 0.0 & 0.0 & 2.5 \\
\hline HAPs & 0.0 & 0.0 & 0.0 & No Source Limit \\
\hline
\end{tabular}

Note: The TA-33-G-1 generator did not operate during 2004.

\begin{tabular}{|c|c|c|c|c|}
\hline Standby Generators & $\begin{array}{c}\text { Jan-June } \\
\text { Emissions } \\
\text { (ton) }\end{array}$ & $\begin{array}{c}\text { July-Dec } \\
\text { Emissions } \\
\text { (ton) }\end{array}$ & $\begin{array}{c}\text { Annual } \\
\text { (ton) }\end{array}$ & Emission Limits \\
\hline TSP & 0.10 & 0.20 & 0.30 & \\
\hline PM & 0.10 & 0.20 & 0.30 & No Source Specific \\
\hline NOx & 2.20 & 3.67 & 5.87 & Emission Limits for \\
Standby \\
Generators \\
\hline CO & 0.50 & 0.86 & 1.36 & \\
\hline VOC & 0.10 & 0.22 & 0.32 & \\
\hline SOx & 0.50 & 0.60 & 1.10 & \\
\hline HAPs & $1.1 \mathrm{E}-03$ & $1.5 \mathrm{E}-03$ & $2.6 \mathrm{E}-03$ & \\
\hline
\end{tabular}

Note: Standby Generators are insignificant sources. Insignificant source information is provided to

demonstrate compliance with PSD applicability thresholds.

2.8.a Paper Shredder

\begin{tabular}{|c|c|c|c|c|}
\hline $\begin{array}{c}\text { Emission Unit } \\
\text { TA-52-11 }\end{array}$ & $\begin{array}{c}\text { January - June } \\
\text { Emissions } \\
\text { (ton) }\end{array}$ & $\begin{array}{c}\text { July - December } \\
\text { Emissions } \\
\text { (ton) }\end{array}$ & $\begin{array}{c}\text { Annual Emissions } \\
\text { (ton) }\end{array}$ & $\begin{array}{c}\text { Emission Limit } \\
\text { (Permit Condition } \\
\text { 2.8.2.1) } \\
\text { (ton per year) }\end{array}$ \\
\hline TSP & 0.0012 & 0.0000 & 0.0012 & 13 \\
\hline
\end{tabular}

Note: The paper shredder was shutdown in July 2004 and was replaced with a new data disintegrator (see data disintegrator - section 2.8.b) 
Los Alamos National Laboratory

2004 Semi-Annual Emissions Report

(July through December)

2.8.b Data Disintegrator (Unit replaced paper shredder)

\begin{tabular}{|c|c|c|c|c|}
\hline $\begin{array}{c}\text { Emission Unit } \\
\text { TA-52-11 }\end{array}$ & $\begin{array}{c}\text { January - June } \\
\text { Emissions } \\
\text { (ton) }\end{array}$ & $\begin{array}{c}\text { July - December } \\
\text { Emissions } \\
\text { (ton) }\end{array}$ & $\begin{array}{c}\text { Annual Emissions } \\
\text { (ton) }\end{array}$ & $\begin{array}{c}\text { Emission Limits } \\
\text { (NSR Permit No. 2195 } \\
\text { H) } \\
\text { (ton) }\end{array}$ \\
\hline TSP & 0.00 & 0.10 & 0.10 & 9.9 \\
\hline PM10 & 0.00 & 0.09 & 0.09 & 9.9 \\
\hline
\end{tabular}

Note: The data disintegrator was started on August 18, 2004, and replaced the existing paper shredder. This unit and

its allowable emissions will be included in the next LANL Title $V$ operating permit modification. The data disintegrator

was installed under Air Quality Permit No. 2195-H. The emissions from this unit are included in the facility wide total.

2.9 Power Plant at Technical Area 3 (TA-3-22)

\begin{tabular}{|l|c|c|c|c|c|}
\hline Pollutant & $\begin{array}{c}\text { January - June } \\
\text { Emissions } \\
\text { (ton) }\end{array}$ & $\begin{array}{c}\text { July - December } \\
\text { Emissions } \\
\text { (ton) }\end{array}$ & $\begin{array}{c}\text { Annual Emissions } \\
\text { (ton) }\end{array}$ & $\begin{array}{c}\text { Emission Limits } \\
\text { (Permit Condition } \\
\text { 2.9.2) } \\
\text { 12 mo. rolling total } \\
\text { (ton) }\end{array}$ & $\begin{array}{c}\text { Emission Limits } \\
\text { (NSR Permit No. } \\
\text { 2195BM1) } \\
\text { 12 mo. rolling total } \\
\text { (ton) }\end{array}$ \\
\hline NOx & 8.85 & 7.49 & 16.34 & 99.6 & 60.2 \\
\hline $\mathrm{SO}_{2}$ & 0.15 & 0.15 & 0.30 & 36.9 & 7.9 \\
\hline $\mathrm{TSP}$ & 1.18 & 1.00 & 2.18 & 15.7 & 8.4 \\
\hline $\mathrm{PM}_{10}$ & 1.17 & 0.99 & 2.16 & 15.7 & 8.2 \\
\hline $\mathrm{CO}$ & 6.10 & 5.16 & 11.26 & 81.3 & 41.3 \\
\hline VOC & 0.83 & 0.70 & 1.53 & 11.1 & 5.6 \\
\hline HAPS & 0.29 & 0.24 & 0.53 & No Source Limit & No Source Limit \\
\hline
\end{tabular}

Note: The allowable emission limits from Air Quality Permit No. 2195BM1 are included above. This permit was issued on July 30, 2004, for

the installation of a combustion tubine. Installion of the tuine is not yet complete. These limits are the most current applicable limits for

the power plant boilers. These new limits will be included in the next LANL Title V operating permit modification.

\subsection{Rock Crusher}

The Rock Crusher was not used in 2004. The unit was retired June 10, 2004 

This report has been reproduced directly from the best available copy. It is available electronically on the Web (http://www.doe.gov/bridge).

Copies are available for sale to U.S. Department of Energy employees and contractors from:

Office of Scientific and Technical Information P.O. Box 62

Oak Ridge, TN 37831

(865) 576-8401

Copies are available for sale to the public from: National Technical Information Service

U.S. Department of Commerce

5285 Port Royal Road

Springfield, VA 22161

(800) 553-6847 


\section{Los Alamos}

NATIONAL LABORATORY

EST.1943 